\title{
Particle Identification with the PANDA Barrel DIRC and the GlueX DIRC
}

\author{
Dissertation \\ zur Erlangung des Doktorgrades \\ der Naturwissenschaften
}

\author{
vorgelegt beim Fachbereich Physik \\ der Johann Wolfgang Goethe-Universität \\ in Frankfurt am Main
}

von

Ahmed Ali

aus Sohag/Ägypten

Frankfurt am Main (2021)

$\mathrm{D}(30)$ 
vom Fachbereich Physik der

Johann Wolfgang Goethe-Universität als Dissertation angenommen.

Dekan: Prof. Dr. Harald Appelshauser

Gutachter: Prof. Dr. Klaus Peters

Prof. Dr. Christoph Blume

Datum der Disputation: 


\begin{abstract}
Next-generation DIRC detectors, like the PANDA Barrel DIRC, with improved optical designs and better spatial and timing resolution, require correspondingly advanced reconstruction and PID methods. The investigation of the PID performance of two DIRC counters and the evaluation of the reconstruction and PID algorithms form the core of this thesis. Several reconstruction and PID approaches were developed, optimized, and tested using hadronic beam particles, experimental physics events, and Geant simulations. The near-final design of the PANDA Barrel DIRC was evaluated with a prototype in the T9 beamline at CERN in 2018. The analysis finds excellent agreement between the experimental data and the Geant simulations for all reconstruction algorithms. The best PID performance of up to $5.2 \pm 0.2$ s.d. $\pi / \mathrm{K}$ separation at $3.5 \mathrm{GeV} / \mathrm{c}$, was obtained with a time imaging PID method. The PANDA Barrel DIRC simulation, as well as the reconstruction and PID algorithms, were evaluated using experimental data from the GlueX DIRC as part of the FAIR Phase-0 program. The performance validation was carried out using physics events of the GlueX experiment and simulations. The initial analysis results of the commissioning dataset show a $\pi / \mathrm{K}$ separation power of up to $3 \mathrm{~s}$.d. at a momentum of 3.0-3.5 GeV/c, obtained using a geometric reconstruction algorithm.
\end{abstract}




\section{Kurzfassung}

DIRC-Detektoren der nächsten Generation, wie der PANDA Barrel DIRC, mit verbesserten optischen Designs und höhere Zeit- und Positionsauflösung der CherenkovPhotonen, erfordern entsprechend fortschrittliche Methoden für die Ereignisrekonstruktion und Teilchenidentifikation (PID). Die Untersuchung der PID-Leistung von zwei neuen DIRC-Zählern und die Evaluierung der Rekonstruktions- und PIDAlgorithmen bilden den Kern dieser Arbeit. Mehrere Rekonstruktions- und PIDAnsätze wurden entwickelt, optimiert und unter Verwendung von hadronischen Teilchenstrahlen mit experimentellen physikalischen Ereignissen und Geant-Simulationen getestet. Das Design des PANDA Barrel DIRC wurde 2018 mit einem Prototyp während einer Strahlzeit am CERN PS untersucht. Die Analyse zeigt eine hervorragende Übereinstimmung zwischen den experimentellen Daten und den GeantSimulationen für alle Rekonstruktionsalgorithmen. Die beste PID-Leistung von bis zu 5,2 \pm , 2 Standardabweichungen (s.d.) für die $\pi / \mathrm{K}$-Trennungbei 3,5 GeV/c wurde mit einer zeitabbildenden PID-Methode erzielt. Im Rahmen des FAIR Phase-0 Programms wurde die für den PANDA Barrel DIRC entwickelte Simulation sowie die DIRC-Rekonstruktions- und PID-Algorithmen an den GlueX DIRC-Detektor angepasst und anhand von physikalischen Ereignissen am GlueX-Experiment evaluiert. Die ersten Analyseergebnisse des Inbetriebnahmedatensatzes zeigen eine $\pi / \mathrm{K}$ Trennleistung von bis zu 3 s.d. im Impulsbereich von 3,0-3,5 GeV/c, wurde mit einem geometrischen Rekonstruktionsalgorithmus erreicht. 


\section{Contents}

1 Introduction and Overview 1

2 The PANDA Experiment $\quad 3$

2.1 FAIR Facility . . . . . . . . . . . . . . . . . . 3

2.2 The PANDA Experiment . . . . . . . . . . . . . . . . 4

3 The PANDA Barrel DIRC $\quad \mathbf{1 5}$

3.1 DIRC Principle . . . . . . . . . . . . . . . . . . . 15

3.2 PANDA PID Requirements . . . . . . . . . . . . 16

3.3 PANDA Barrel DIRC Overview . . . . . . . . . . . . . . . 18

3.3.1 The PANDA Barrel DIRC Design . . . . . . . . . . . . . . . 19

3.4 PANDA Barrel DIRC Simulation and PID Performance . . . . . . . . 20

3.4 Geometrical Reconstruction . . . . . . . . . . . . 21

3.4 Time Imaging . . . . . . . . . . . . . . . . . . . . . . 25

3.5 The PANDA Barrel DIRC Prototype . . . . . . . . . . . . . . . 28

3.5.1 Prototype Simulation Aspects . . . . . . . . . . . . . . . 28

3.5.2 Summary ..................... 35

4 The PANDA Barrel DIRC Prototype Test Beam 37

4.1 Introduction . . . . . . . . . . . . . . . . . 37

4.2 Test Beam Setup . . . . . . . . . . . . . . . . . . 37

4.2 .1 Test Beam Facility . . . . . . . . . . . . . . . . . 37

4.2 .2 Prototype Setup . . . . . . . . . . . . . . . . . . 38

4.3 Event Selection and Particle Tagging . . . . . . . . . . . . . . . 39

4.4 Hit Pattern . . . . . . . . . . . . . . . . . . . . . . . . . 41

4.5 Timing Calibrations . . . . . . . . . . . . . . . . . 43

4.6 PID Performance . . . . . . . . . . . . . . . . 47

4.7 Geometrical Reconstruction . . . . . . . . . . . . . . . . 47

4.7.1 Event Track Hit Selection . . . . . . . . . . . . . . . . . . 48

4.7.2 Determination of the Cherenkov Angle Resolution per Photon 49

4.7 .3 Photon Yield . . . . . . . . . . . . . . . 51

4.7 .4 PID Performance . . . . . . . . . . . . . . . 51

4.7.5 PID Performance Optimization . . . . . . . . . . . 51

4.7.6 Geometrical Reconstruction Second Approach . . . . . . . . . 56

4.8 Error Calculations . . . . . . . . . . . . . . . . . 57

4.8.1 Separation Power . . . . . . . . . . . . . 57

4.8.2 Single Photon Resolution . . . . . . . . . . . . . . . . 60

4.8.3 Photon Yield . . . . . . . . . . . . . . . . . 62

4.8.4 Comparison Between Beam Data and Simulation . . . . . . . 64 
4.8.5 Cherenkov Angle Data PDF . . . . . . . . . . . . . 66

4.8 .6 Time-based Imaging . . . . . . . . . . . . . . . . . 68

4.9 Azimuthal Angle Study . . . . . . . . . . . . . . . . . . 70

4.10 Summary . . . . . . . . . . . . . . . . . . . . 75

$\begin{array}{lll}5 & \text { The GlueX DIRC } & 77\end{array}$

5.1 Introduction . . . . . . . . . . . . . . . . . . 77

5.2 Detector Construction and Installation . . . . . . . . . . . 79

5.2 .1 Bar Boxes . . . . . . . . . . . . . . . . 79

5.2 .2 Expansion Volume . . . . . . . . . . . . . . . . 81

5.2.3 Photon Detection and Readout . . . . . . . . . . . . . 83

5.2 .4 Calibration System . . . . . . . . . . . . . . 83

5.3 The GlueX DIRC Simulation and Reconstruction . . . . . . . . . . . 84

5.3.1 Reconstruction and PID methods . . . . . . . . . . . . . 84

5.4 Preliminary Commissioning Results . . . . . . . . . . . . . . 86

5.4.1 PID Calibration Physics Channels . . . . . . . . . . . . 86

5.4 .2 Hit Pattern . . . . . . . . . . . . . . . . . . . 87

5.4.3 Cherenkov Angle Reconstruction . . . . . . . . . . . . 88

5.4 .4 Photon Yield . . . . . . . . . . . . . . . . . . . 92

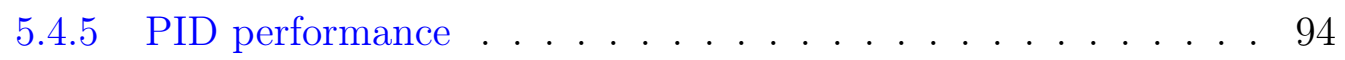

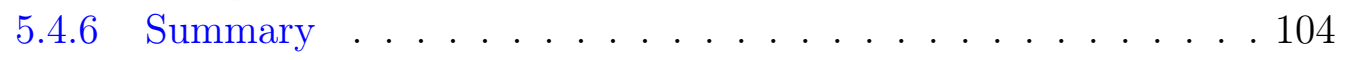

6 Conclusion and Outlook $\quad 107$

$\begin{array}{lll}7 & \text { Ausführliche Zusammenfassung } & 109\end{array}$ 


\section{Chapter 1}

\section{Introduction and Overview}

The PANDA experiment at the international accelerator Facility for Antiproton and Ion Research in Europe (FAIR) near GSI, Darmstadt, Germany, will address fundamental questions of hadron physics. It is designed to study hadronic interactions using high-intensity cooled antiproton beams with momenta up to $15 \mathrm{GeV} / \mathrm{c}$ and internal fixed target. The PANDA detector is designed to cover nearly a $4 \pi$ solid angle around the interaction point, with several hermetic layers of sub-detectors. This is done mainly by a solenoid and dipole magnets, tracking detectors, particle identification (PID) detectors, calorimetry detectors, and muon detectors. A detailed overview of the PANDA spectrometer, including all sub-detectors, will be given in chapter 2 .

Excellent particle identification for a wide range of momenta and the full solid angle is required to achieve the PANDA physics goals. For this purpose, a DIRC counter with a cylindrical shape will deliver hadronic particle identification in the barrel region of the PANDA target spectrometer. The Barrel DIRC covers the polar angle range $22^{\circ} \leq \theta \leq 140^{\circ}$. It is designed to provide $\pi / \mathrm{K}$ separation for momenta up to $3.5 \mathrm{GeV} / c$ with a separation power of at least 3 standard deviations. The design of the PANDA Barrel DIRC was inspired by the successful BABAR DIRC counter with several key improvements, such as fast timing, lens focusing, and a compact expansion volume.

Future DIRC detectors, like the PANDA Barrel DIRC, with cutting-edge optical designs and improved spatial and timing resolution, require correspondingly advanced reconstruction and PID algorithms. Several reconstruction algorithms were developed and optimized to determine the detector figures of merits. The "geometrical reconstruction" determines the Cherenkov angle by relying primarily on the position of the detected photons. The "time imaging," however, utilizes both position and time measurements by directly performing the maximum likelihood fit. The figures of merits of the detector were quantified in terms of single photon Cherenkov angle resolution, photon yield, and $\pi / \mathrm{K}$ separation power.

The advanced design of the Barrel DIRC, as well as the reconstruction and PID methods, required experimental validation. To this end, the key performance parameters of increasingly complex prototypes were determined during several test beam campaigns at GSI and CERN. The latest test beam campaign was performed 
at CERN in 2018 to validate the near-final design.

In the scope of this thesis, the reconstruction and PID algorithms were developed, tested, and optimized, to study the PID performance of the 2018 DIRC prototype using hadronic particle beams at the CERN PS and Monte Carlo simulation. Chapter 3 presents the Monte Carlo simulation predictions and the analysis of the beam data is described in chapter 4 .

The thesis work also includes investigation of the GlueX DIRC PID performance. The PANDA Barrel DIRC simulation and reconstruction algorithms were implemented in the GlueX experiment at Jefferson Lab to apply lessons learned to PANDA, as part of the FAIR Phase-0 program. The GlueX experiment, located in Hall D at JLab, utilizes a linearly polarized $9 \mathrm{GeV}$ photon beam hitting a proton fixed target to conduct a search for exotic hybrid mesons. To enhance the PID capabilities, a DIRC counter was installed in the forward region of the experiment. The simulation, as well as the reconstruction and PID methods, were validated using kinematically identified $\pi^{ \pm}$and $\mathrm{K}^{ \pm}$from $\rho$ and $\phi$ decays. Chapter 5 provides an overview of the GlueX spectrometer and discusses the particle identification performance of the GlueX DIRC using the commissioning data and Monte Carlo simulation. 


\section{Chapter 2}

\section{The PANDA Experiment}

\section{$2.1 \quad$ FAIR Facility}

The Facility for Antiproton and Ion Research (FAIR), shown in Fig. 2.1, is a new accelerator complex, currently under construction at the GSI facility in Germany near Darmstadt [1]. The existing GSI accelerators will serve as a particle injectors for FAIR. The UNILAC (UNIversal Linear ACcelerator) is a linear accelerator with around $120 \mathrm{~m}$ length and serves as the first stage in the acceleration chain, by accelerating ions up to $13-15 \mathrm{MeV}$ per nucleon [1].

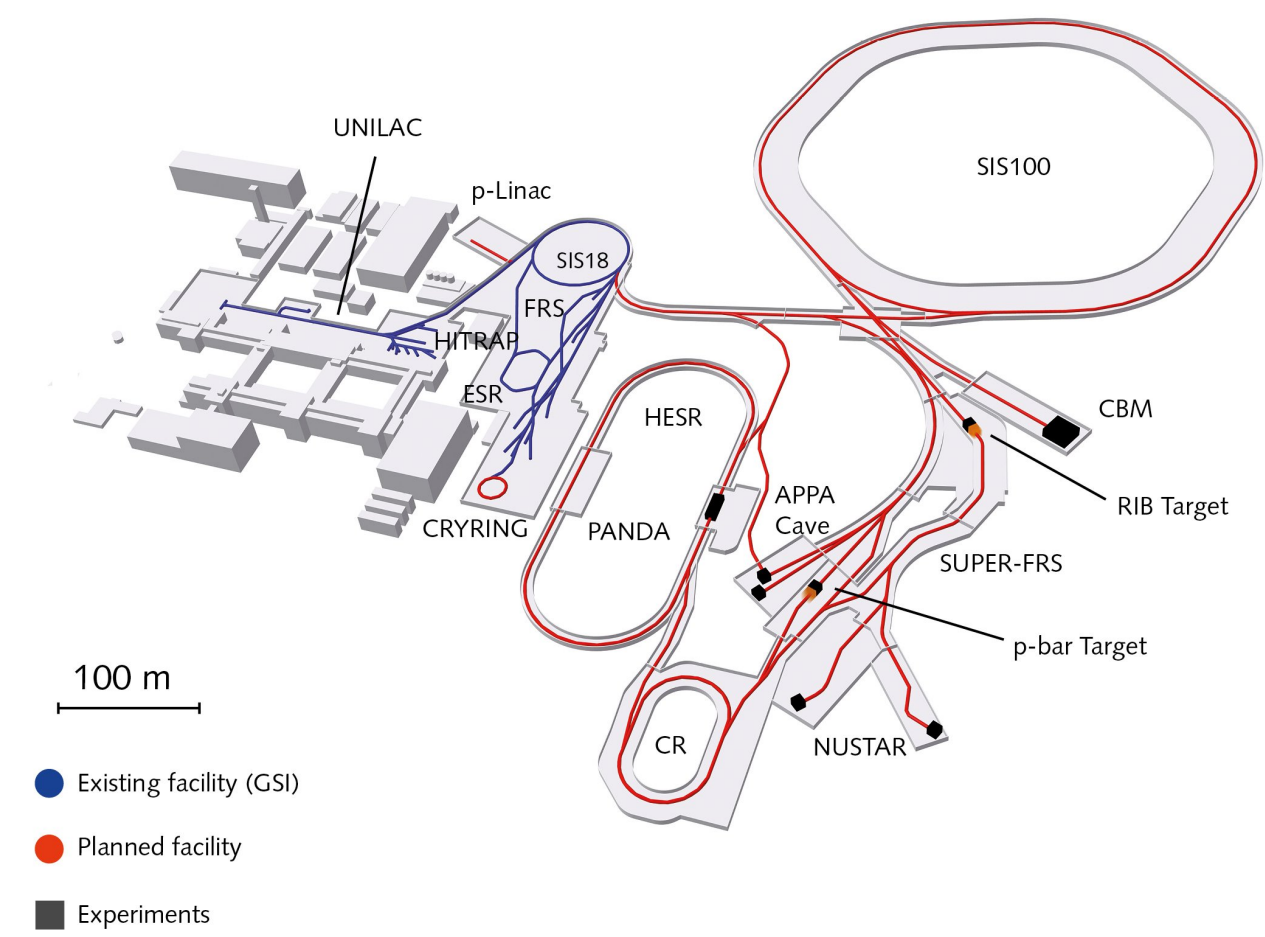

Figure 2.1: Schematic of the FAIR layout incorporating the current GSI facilities (blue) in the future installations (red) [1].

Ions coming from the linear accelerator UNILAC are injected into the ring accelerator SIS18 [2], the first synchrotron in the accelerator chain at GSI, with a circum- 
ference of $216 \mathrm{~m}$. Here they can be accelerated to even higher speed. It accelerates light ions up to $2 \mathrm{GeV} / \mathrm{u}$, heavy ions up to $1 \mathrm{GeV} / \mathrm{u}$ and protons up to $4.7 \mathrm{GeV}$. To provide high intensity proton beams, an additional linear accelerator is planned to be installed upstream. The p-Linac (Proton LINear ACcelerator) pre-accelerates protons before they enter the SIS18. The FAIR accelerator complex includes a new synchrotron ring SIS100 [2] with $1100 \mathrm{~m}$ circumference. It is designed to perform fast acceleration of high-intensity ion and proton beams. In particularly, it will accelerate up to $4 \cdot 10^{13}$ protons per pulse to a beam momentum of $30 \mathrm{GeV} / \mathrm{c}$ with a cycle time of 2 seconds. A collision of these highly energetic protons with a copper target will produce antiprotons that can be distinguished from various other generated particles by a magnetic horn. The next stage is the fast cooling of the antiprotons in the Collector Ring (CR). The antiprotons are then delivered to the High Energy Storage Ring (HESR) see, Fig. 2.2. In the HESR [3], a racetrack-shaped storage ring, the antiprotons can be delivered in a momentum range between 1.5 and $15 \mathrm{GeV} / \mathrm{c}$ [1]. The HESR has a circumference of $575 \mathrm{~m}$ and can provide antiporon beams run in two modes: high resolution and high luminosity. The high resolution mode can be used for scanning resonances with a given antiproton momentum distribution, which has about $\Delta p / p \sim 4 \cdot 10^{-5}$ momentum uncertainty. About $10^{10}$ antiprotons can be stored in this mode and a luminosity of approximately $2 \cdot 10^{31} \mathrm{~cm}^{-2} \mathrm{~s}^{-1}$ can be achieved. In the high luminosity mode the luminosity is enhanced by a factor of 10 with lower momentum resolution of about $\Delta p / p \sim 10^{-4}$. In the case of rare events that require high statistics, this mode should be selected.

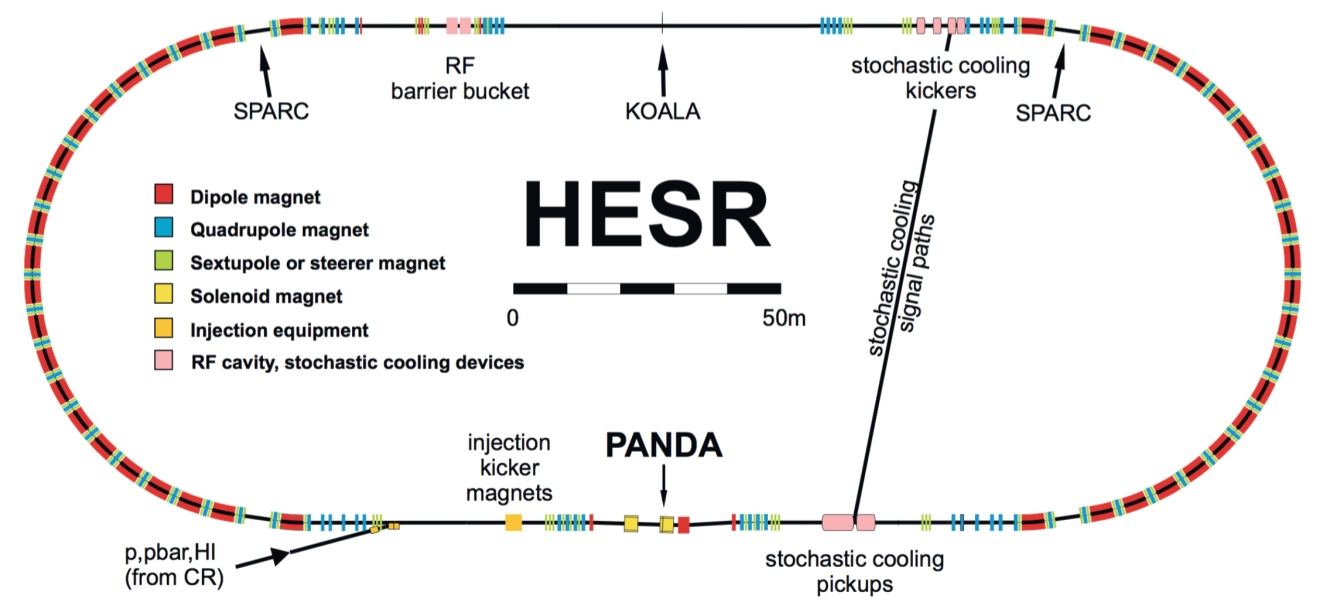

Figure 2.2: Schematic drawing of the HESR [3].

\subsection{The PANDA Experiment}

PANDA [4, 5] is a fixed target $\bar{p} p$ experiment at HESR, it is one of the key experiments at the FAIR accelerator complex, where many physics studies can be addressed. In general, the $\bar{p} p$ collisions have the advantage of achieving different physics reactions rather than pp or $e^{+} e^{-}$collisions, due to vanishing the baryon number and the total charge. 
The PANDA detector features two spectrometers. Figure 2.3 shows a schematic drawing of the detector according to the planned design. The PANDA Target Spectrometer (TS) is designed with almost $4 \pi$ angle coverage. Because PANDA is a fixed target experiment, the kinematics require that the reaction products favor the forward direction and the Forward Spectrometer (FS) is designed for that purpose; accordingly, the detector has an asymmetric shape relative to the polar angle. Both spectrometers contain several types of detector systems for tracking, calorimetry, and PID purposes. A full description of the PANDA detector is given in Ref. [4].

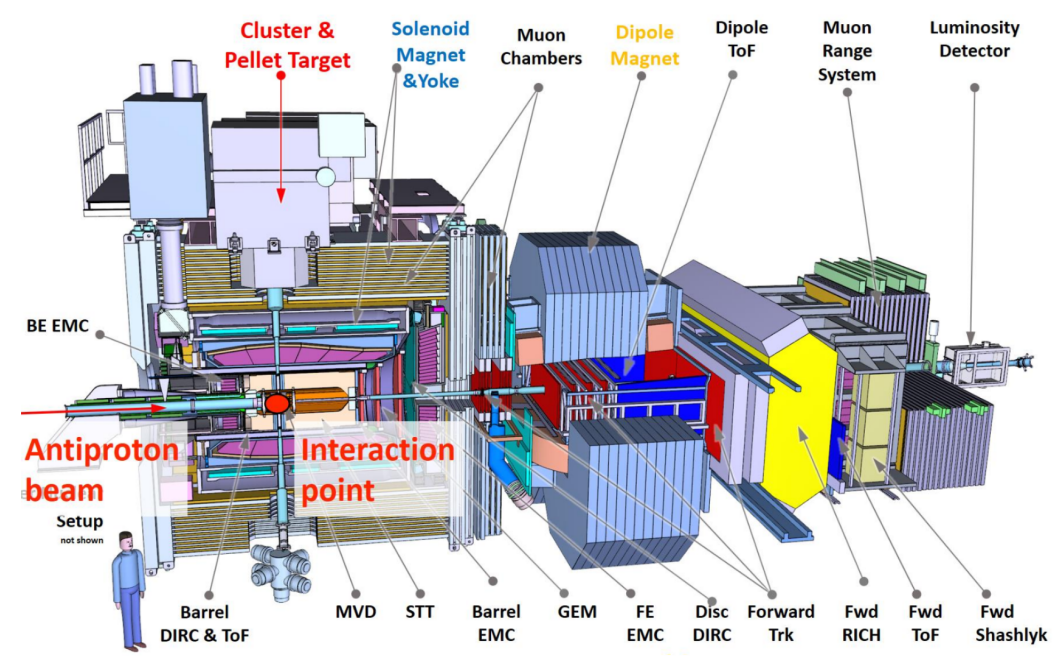

Figure 2.3: The PANDA detector, target and forward spectrometers are shown [4].

\subsubsection{Hadron Physics with the PANDA Experiment}

The PANDA experiment aims to extend our understanding of strong interaction, described by the field theory of Quantum Chromodynamics (QCD), by conducting high-precision measurements and covering the charmonium mass region (see Fig. 2.4). Most high precision measurements are presently conducted at $e^{+} e^{-}$colliders, such as BESIII and Belle II. Since the initial $e^{+} e^{-}$system is well-known, these collider experiments can offer a straight-forward production mechanism and provide high resolution measurements of the final state particles. However, these experiments are basically restricted to initial spin-parities of $J^{P C}=1^{--}$, which limits the access to characteristics of other spin states. In contrast, the usage of antiprotonproton annihilation does not suffer from this limitation. Together with an excellent energy resolution, down to around $25 \mathrm{keV}$, this kind of reactions gives a complementary and unique opportunity to conduct hadron and charmonium spectroscopy in that energy range. The PANDA physics program [6, 4] covers multiple aspects of Quantum Chromodynamics (QCD):

- Charmonium and open charm spectroscopy with precision measurements of mass, width, and decay branching ratios;

- Exotic bound states such as hybrid excitations, glueballs and other exotic configurations like multiquark states;

- Baryon spectroscopy in the strange and charm quark sector; 


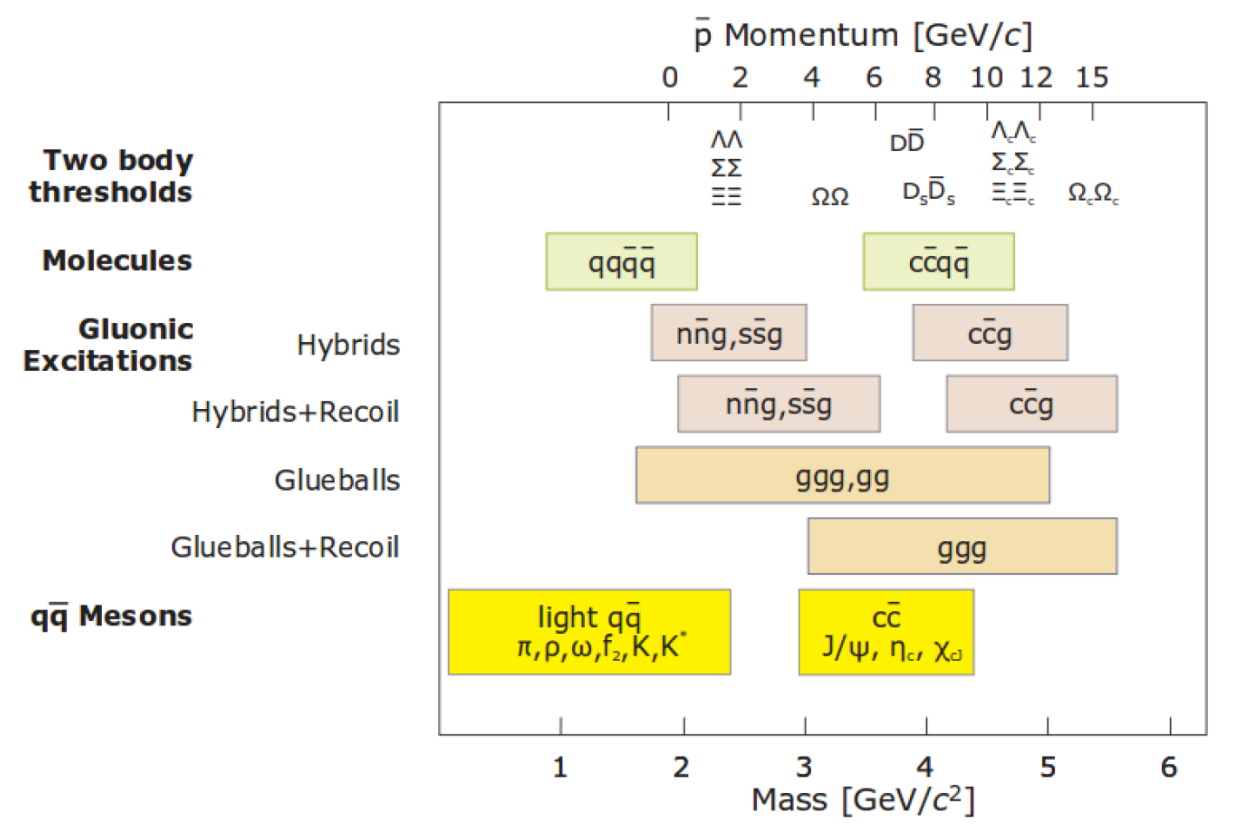

Figure 2.4: Mass range of hadrons that will be accessible at PANDA. The upper scale indicates the corresponding antiproton momenta available in the fixed-target experiment. The HESR will provide 1.5 to $15 \mathrm{GeV} / \mathrm{c}$ antiprotons, allowing for charmonium spectroscopy, the search for charmed hybrids and glueballs, the production of D meson baryon pairs as well as hypernuclear studies [7].

- Hadrons in nuclear matter;

- Hypernuclear physics.

A comprehensive summary report on the physics program of the PANDA experiment can be found in Ref. [8, 6, 4]. To achieve the scientific goals of the PANDA physics program, it is essential to detect and identify final state particles, particularly charged kaons. The key challenge is to separate these signal events from the huge pionic background. Therefore an excellent particle identification is required, while keeping the whole PANDA spectrometer as hermetic and compact as possible.

\subsubsection{Target System}

The selection of an effective target system is challenging because of the space constraint of the target spectrometer [9, 10]. A target density of about $4.10^{15}$ hydrogen atoms per $\mathrm{cm}^{2}$ is required to achieve the highest luminosity. Two target systems were designed for the cluster-jet and the pellet targets. The former is already completely developed and will be operated at the day-1 PANDA for commissioning and medium luminosity experiments. The frozen pellet target can be used to achieve higher effective target densities. The pellet [9] target provides a stream of small drops of frozen hydrogen with a typical diameter between $10 \mu \mathrm{m}$ and $30 \mu \mathrm{m}$, created in a triple point chamber. these droplets are injected into the target tube and transmitted to the beam pipe to interact with the antiprotons, see Fig. 2.5a. The dropping speed is about $60 \mathrm{~m} / \mathrm{s}$ at a flow rate of 100,000 pellets per second. This target is preferable to achieve high luminosities. However, it is important to provide the droplets at a constant rate to minimize the chance of the target density to 
become inhomogeneous, which would cause luminosity variations.

For the cluster-jet target [10], see Fig. 2.5b, either hydrogen $\left(H_{2}\right)$ or deuterium $\left(D_{2}\right)$ or other elements can be used used as target material. In case of hydrogen, a single cluster-jet contains around $10^{5}$ atoms. The main working principle is the jets are formed when a pre-cooled gas at a temperature $30 \mathrm{~K}$ is compressed into the beam pipe nozzle of PANDA through a very small diameter, between $10-100 \mu \mathrm{m}$. A sequence of valves and collimators is used to monitor and control the spread of the clusters to obtain the optimal density and quality of the clusters needed for the PANDA physics program.

(a)

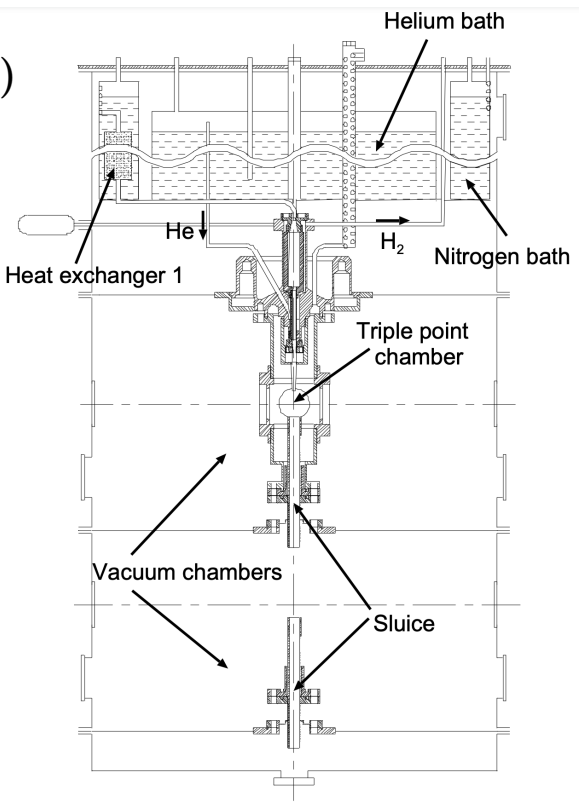

(b)

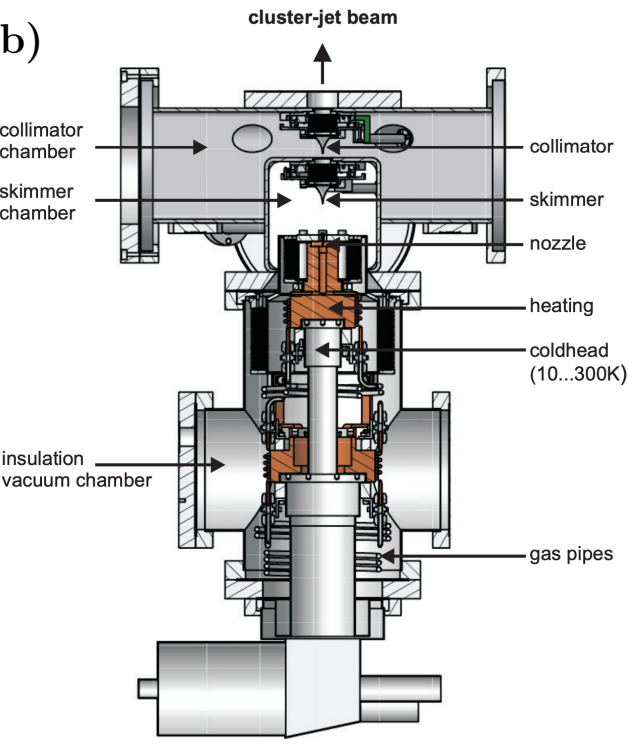

Figure 2.5: The target systems for PANDA. (a) the pellet target system (b) the cluster-jet target system [7].

The created cluster jets move with supersonic speed during the condensation phase. The cluster jets properties can be tuned to achieve the required luminosity by adjusting their size, speed, and density. The cluster size contains between $10^{3}$ to $10^{5}$ atoms per cluster.

\subsubsection{Solenoid Magnet}

A strong magnetic field is essential to perform momentum reconstruction and subsequent particle identification for charged particles. In the TS a superconducting solenoid will be installed. The solenoid will provide a $2 \mathrm{~T}$ magnetic field over a length of about $4 \mathrm{~m}$ and a diameter of $1.9 \mathrm{~m}$. For commissioning and maintenance, the whole magnet system will be mounted on a movable platform to move it out of the beamline; further information can be found in Ref. [11]. 


\subsubsection{Tracking Detectors in the TS}

The tracking in the TS is provided by three detectors: The Micro Vertex Detector, Straw Tube Tracker and Gas Electron Multiplier stations, ordered by their distance from the interaction point. The tracking system is going to provide the track hits created by charged particles. Additionally, tracking information is required for some other detectors, such as the Barrel DIRC and the Endcap Disc DIRC, which provide PID information.

\section{Micro Vertex Detector}

The Micro-Vertex-Detector (MVD) [12] is located in the target spectrometer and is the most adjacent detector to the interaction point, see Fig. 2.6. The MVD is a tracking detector for charged particles and, thus, necessary for a very precise determination of secondary decay vertices of short-lived particles such as hyperons or mesons with charm or strangeness content. The information about both, primary and secondary vertices will be obtained by the MVD, a silicon pixel detector with an vertex resolutions of about $50 \mu \mathrm{m}$ in transverse and $100 \mu \mathrm{m}$ along the beam direction. This detector consists of 4 barrel sections and 6 disks placed around the interaction point. It is necessary to keep the material budget as small as possible since this detector is the innermost of a set of other PANDA sub-detectors. In addition, the detector and the readout system must achieve high radiation hardness. A detailed technical description can be found in Ref. [12].
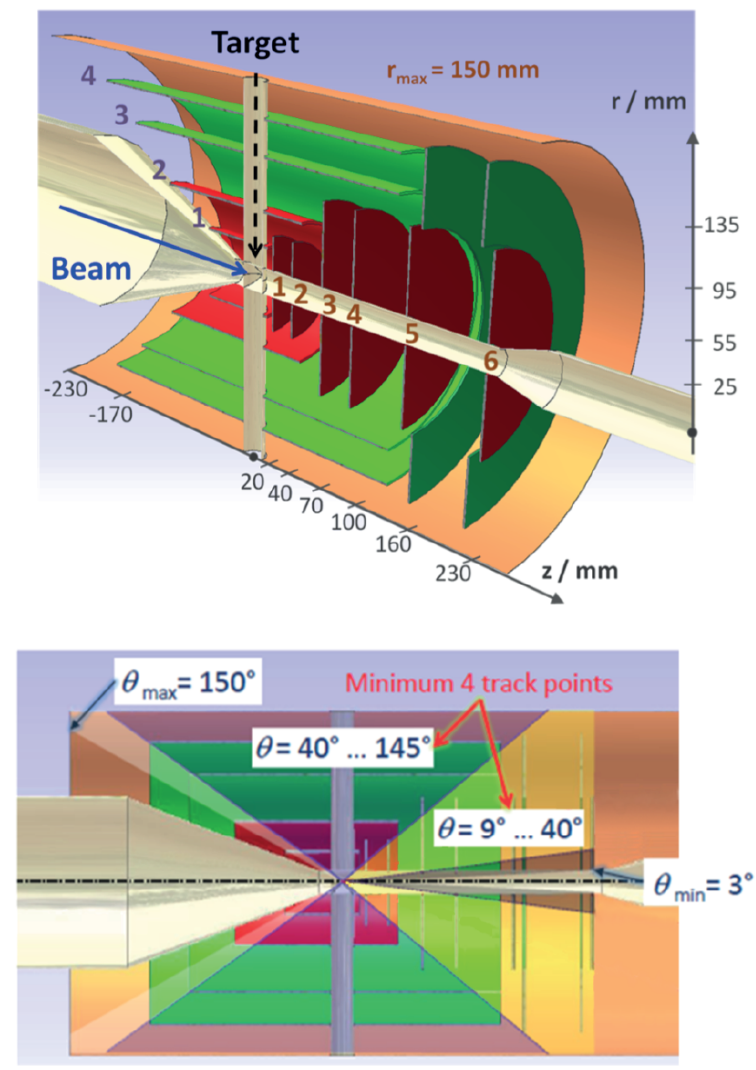

Figure 2.6: 3D drawing of the PANDA Micro Vertex Detector [12]. 


\section{Straw Tube Detector}

Surrounding the MVD, the Straw Tube Tracker (STT) consists of about 45000 drift tubes, the diameter of a single tube is $10 \mathrm{~mm}$, serve as proportional counters filled with gas mixture Ar- $\mathrm{CO}_{2}$ and a gold plated tungsten-rhenium wire with a diameter of $20 \mu \mathrm{m}$ is used as anode inside each tube. The STT has a cylindrical shape with an inner radius of $15 \mathrm{~cm}$, an outer radius of about $42 \mathrm{~cm}$, an overall length of about $1.5 \mathrm{~m}$, see Fig. 2.7. Several double layers and skewed layers arrangement of the tube along the beam axis, allow for a $3 \mathrm{D}$ reconstruction of the charged particle trajectories, providing transverse and longitudinal resolution of about $150 \mu \mathrm{m}$ and $1 \mathrm{~mm}$, respectively, and will also perform PID for momenta below $0.8 \mathrm{GeV} / \mathrm{c}$ using the energy loss measurements $(\mathrm{dE} / \mathrm{dx})$ in the tubes. A detailed technical description of the STT is found in Ref. [13].

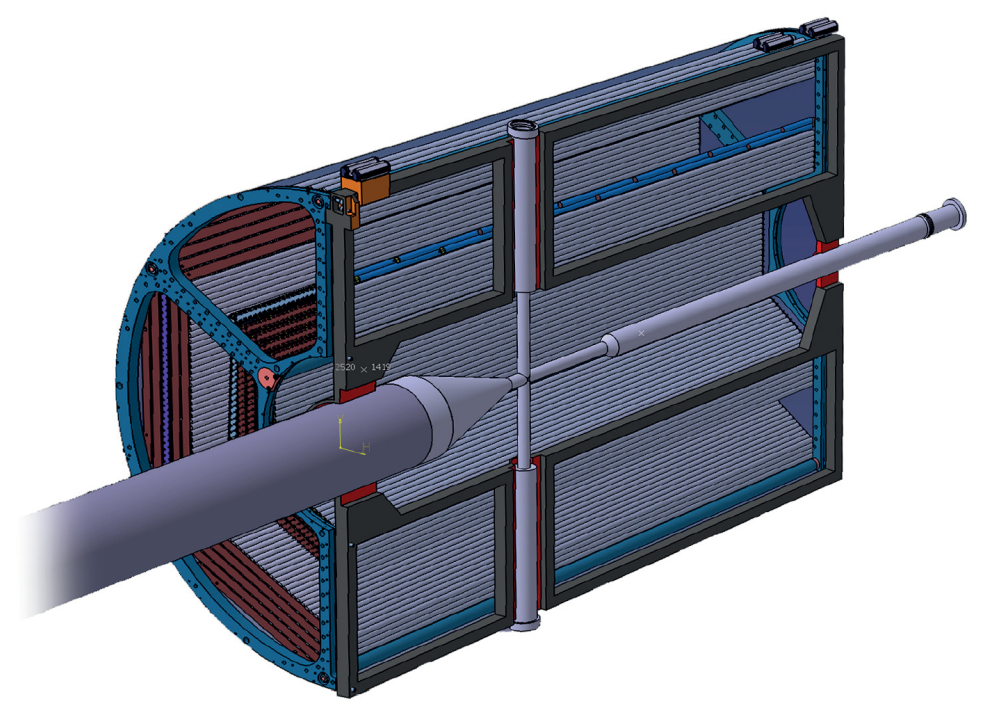

Figure 2.7: Schematic drawing of the PANDA Straw Tube Tracker. Only one half cylinder of the STT is shown. It encloses the beam pipe and a small opening in the top allows for the insertion of the target [13].

\section{GEM Detector}

The Gas Electron Multiplier (GEM) detector is placed at the forward endcap of the PANDA TS and consists of 3 stations covering the polar angle range from $5^{\circ}$ to $22^{\circ}$, see Fig. 2.8. Each GEM station contains 3 GEM foils made from a thin Kapton layer of $50 \mu \mathrm{m}$, with thin copper layers on both sides. The GEM foil stack is housed between a drift cathode and a readout board. By applying a high voltage potential, typically a few $\mathrm{keV} / \mathrm{cm}$, a strong electric field around the holes is created. Electrons generated by ionizing particles will be accelerated along the electric field and create an avalanche inside the GEM holes and the charge will be collected by the anode. The electric signal will be delivered to the readout plane, which is subdivided into approximately 35,000 copper pads. This division allows precise position measurements of the charge cloud, and gives the tracking information of particles through the GEM. The GEM detectors determine particle trajectory positions with a resolution of better than $100 \mu \mathrm{m}$. 


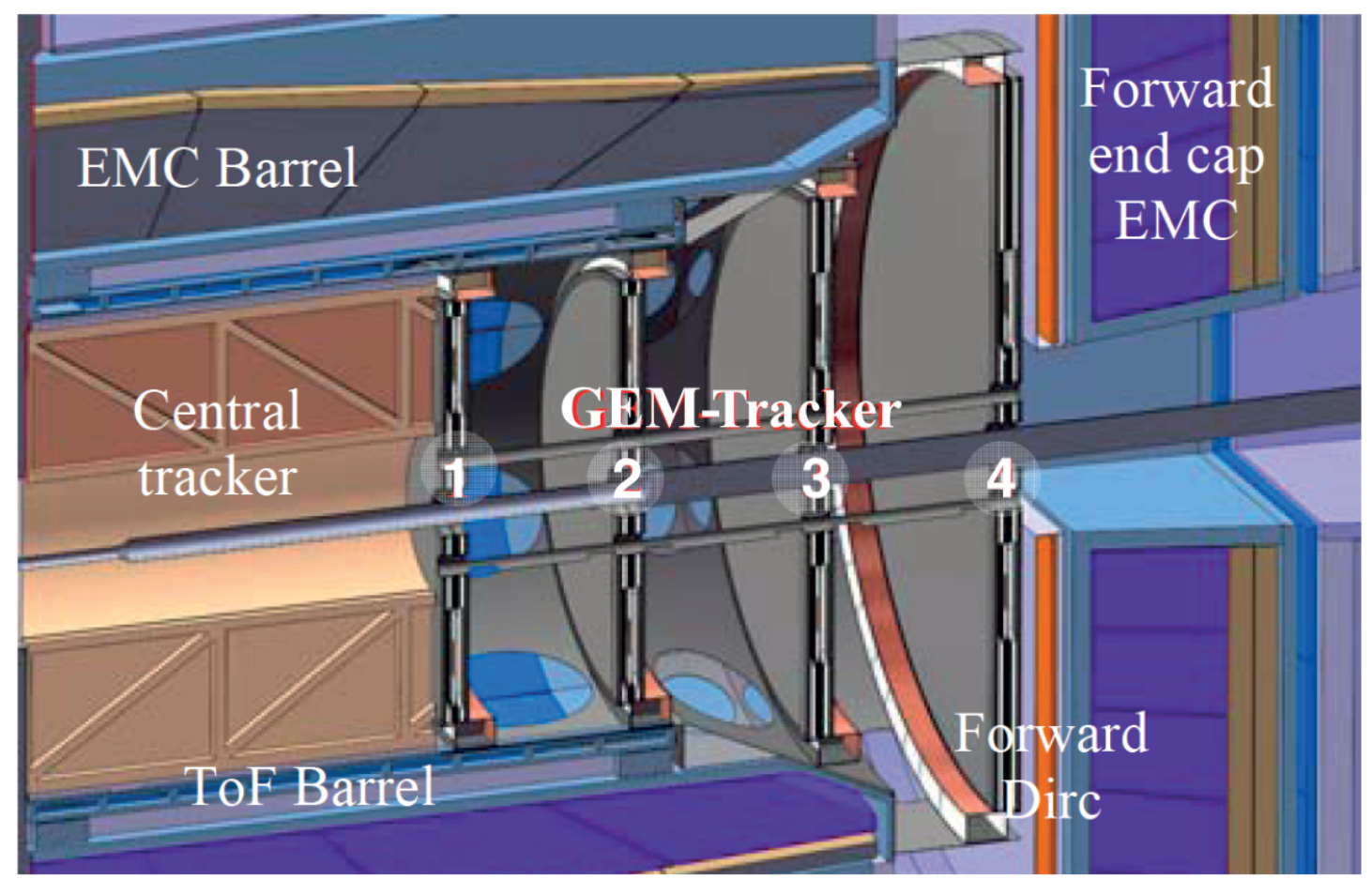

Figure 2.8: Schematic drawing of the GEM detector. Four discs are shown. Discs 2, 3 and 4 are the currently planned stations. Disc 1 is foreseen in the future.

\subsubsection{Electromagnetic Calorimeter}

The primary purpose of a calorimeter is to determine the particle energy by interactions in a special absorber medium and reconstruct the total energy deposited inside that medium. A charged particle passing through a crystal produces bremsstrahlung radiation, which is then transformed into electron-positron pairs. These generated particles emit photons through bremsstrahlung radiation that creates a cascade of secondary particle showers. This mechanism continues until the primary particle has lost all the energy within the crystal, or has passed through. In the PANDA electromagnetic calorimeter (EMC) is subdivided into three parts: a barrel part and two endcaps, the forward and the backward endcap. Due to the compact design of the EMC and high count rates in the Target Spectrometer, a short radiation length and a fast scintillator material are required to construct the EMC. The lead tungstate crystal will be used as crystal material. The barrel calorimeter consists of 11360 crystals, the forward part will contain 3,600 crystals and the backward part will hold 592. This leads to a coverage of about $99 \%$ of the full solid angle and cover the energy range between $10 \mathrm{MeV}$ and $15 \mathrm{GeV}$. A 3D sketch of the EMC is shown in Fig. 2.9. The expected energy resolution for the barrel EMC is below $2 \%$ for particle energies in the order of $1 \mathrm{GeV}$. Further, the EMC will also provide a good intrinsic timing resolution below 1 ns. A complete description of the EMC can be found in Ref. [14].

\subsubsection{PID Detectors in the TS}

In addition to tracking charged particles using different complimentary sub-detectors, particle identification (PID) is essential for event reconstruction. Accordingly, the 


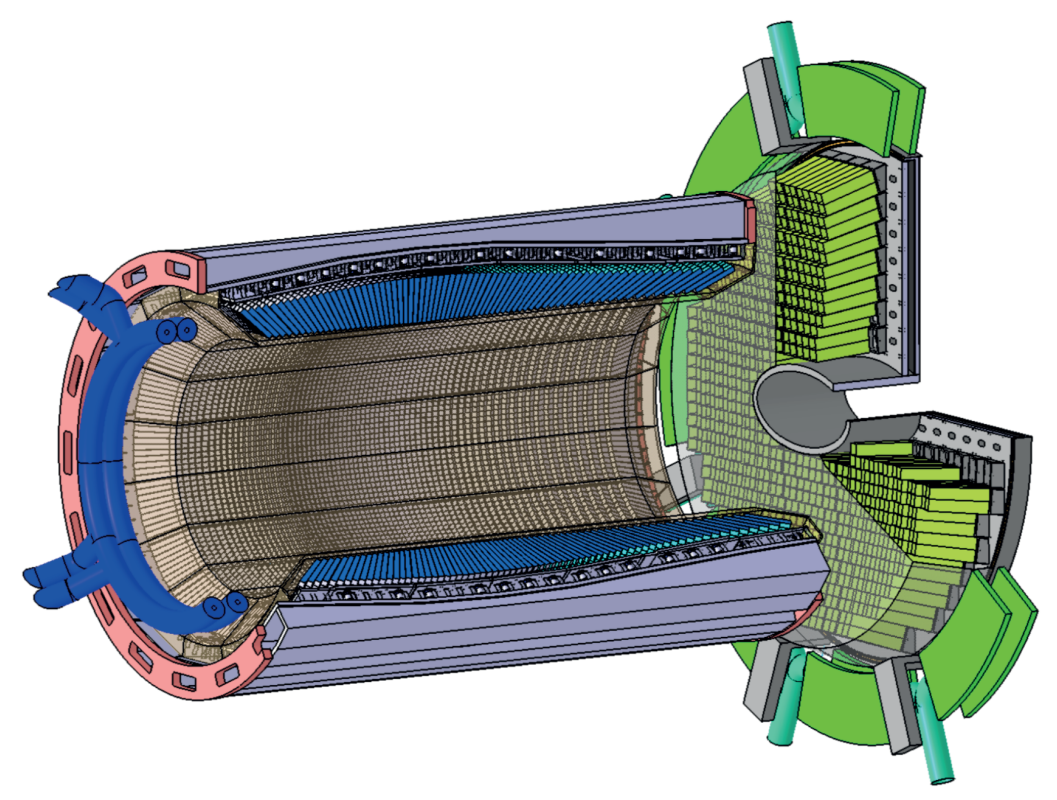

Figure 2.9: Schematic drawing of the electromagnetic calorimeter of the PANDA target spectrometer [14].

design of the PANDA detector incorporates sub-detectors for the PID: Cherenkov detectors, namely the Detection of Internal Reflected Cherenkov Light (DIRC), the Barrel Time of Flight detector, and the Muon Detector System (MDS). In this section, an overview of the different detectors for PID is discussed.

\section{DIRC Counters}

Using the Detection of Internally Reflected Cherenkov light (DIRC), two DIRC detectors will be placed in the target spectrometer. Both of them will provide efficient and clean PID. The barrel DIRC [15] detector will be installed outside the STT, about $48 \mathrm{~cm}$ away from the interaction point, and will cover a polar angle range between $22^{\circ}$ and $140^{\circ}$, to provide $\pi / \mathrm{K}$ separation for momenta up to 3.5 $\mathrm{GeV} / c$ with a separation power of at least 3 standard deviations. The Endcap Disc DIRC (EDD) [16] with an acceptance range between $5^{\circ}$ and $22^{\circ}$ polar angle, between the barrel DIRC and the forward spectrometer. The expexted performance of the EDD is to provide $\pi / \mathrm{K}$ separation for momenta up to $4 \mathrm{GeV} / c$ with a separation power of at least 3 standard deviations. The basic working principle for both subsystems is the measurement of Cherenkov light generated by a charged particle transversing a medium with a velocity exceeding the speed of light in that medium. The Cherenkov photons undergo internal reflections inside the radiator. An array of photodetectors is used to detect the Cherenkov photons. By combining the charged track direction, using the tracking system, with the with the time and location of the detected Cherenkov photons, the Cherenkov angle can be reconstructed, and hence the particle species can be deduced. Figure 2.10 shows a sketch of the two DIRC systems and points out their placement within the PANDA detector. The radiators for both detectors are made from fused silica either in the form of bars as for the barrel DIRC, or in the form of a discs, as for the Endcap Disc DIRC. A comprehensive description of the DIRC Counters can be found in Ref. [15, 16]. 


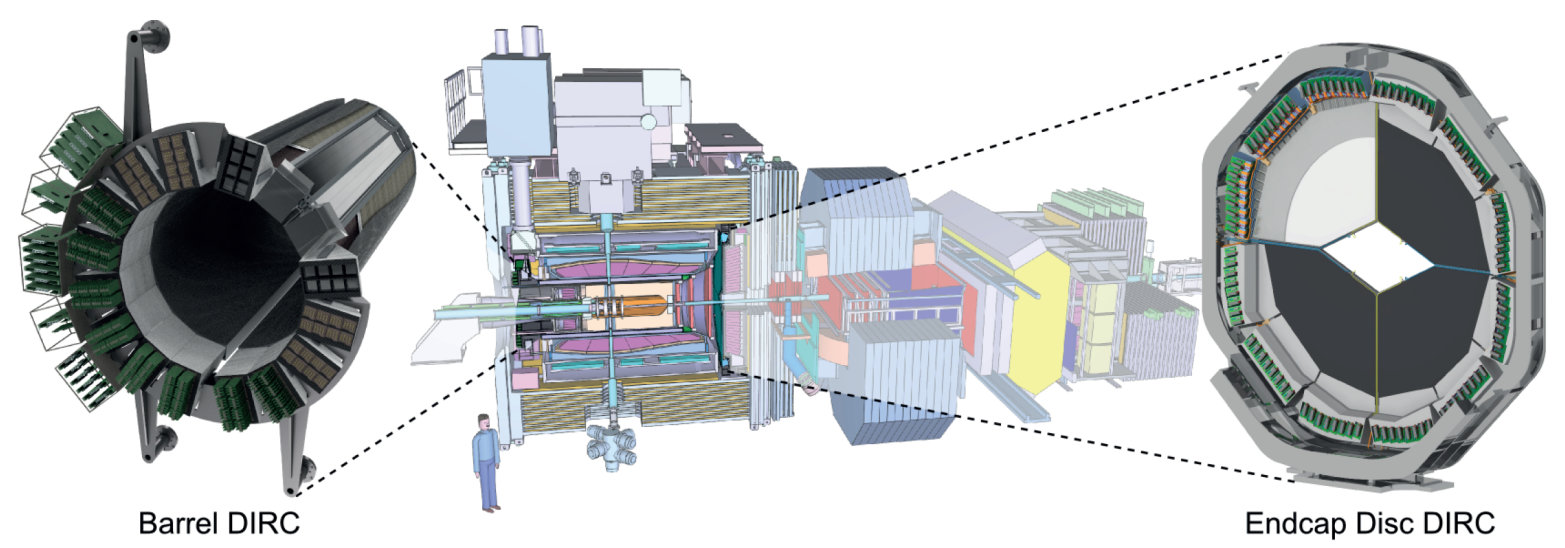

Figure 2.10: The Barrel DIRC and Endcap Disc DIRC layout in PANDA [5].

\section{Barrel Time of Flight}

The Barrel Time-of-Flight (BToF) detector is one of the particle identification subsystems. A CAD drawing of the BToF, including a magnified sketch of a pair of scintillator tiles, is illustrated in Fig. 2.11. The primary goal of the BToF is to measure timing information of the charged particles in the polar angle range between $22^{\circ}$ and $140^{\circ}$, which is used to perform PID of the charged particles with momenta below $0.7 \mathrm{GeV} / \mathrm{c}$. The BToF will be placed in the TS between the Barrel DIRC detector and the EMC. Since the BToF will share the mechanical support with the Barrel DIRC, it will consist of 16 sections, same as the Barrel DIRC. The detector has a low material budget to reduce the effect of the material on the traversing particles until they reach the EMC. The detector consists of scintillating tiles. Each segment has $87 \mathrm{~mm}$ long and $20 \mathrm{~mm}$ thick and is called a super-module. The super-module comprises 120 scintillating tiles, ordered in two columns of 60 scintillators and read out by silicon photomultipliers (SiPM). Generally, the time-of-flight PID technique is based on measuring the flight time of a charged track. The expected travel time for each particle hypothesis with a given momentum can be calculated. By comparing the measured arrival time by the TOF system with the calculated arrival time, the particle species can be deduced. This method of using the flight time to identify particles becomes less efficient as the particle momenta exceed $0.7 \mathrm{GeV} / \mathrm{c}$. Therefore, the BToF is complementary to the Barrel DIRC, which is designed to perform PID up to $4 \mathrm{GeV} / \mathrm{c}$ momentum. The time resolution of the BToF will be around $100 \mathrm{ps}$. The high time precision achieved allows the detector to be used for multiple tasks, including event triggering and particle identification. A complete description of the BToF can be found in Ref. [17].

\section{Muon Detector System}

A Muon Detection System will be installed in the PANDA detector for muon identification up to $10 \mathrm{GeV} / \mathrm{c}$ momentum. The identification is carried out using pattern recognition. The locally reconstructed track segments will be matched with the reconstructed tracks in the other subsystems inside the magnets to perform a global reconstruction. The TS Muon System will include three parts: a barrel part that is inserted within the iron yoke, a barrel forward end-cap downstream from the primary vertex, and a removable muon filter will be located between the TS and the dipole magnet in the FS, see Fig. 2.12. All parts are constructed by alternating de- 


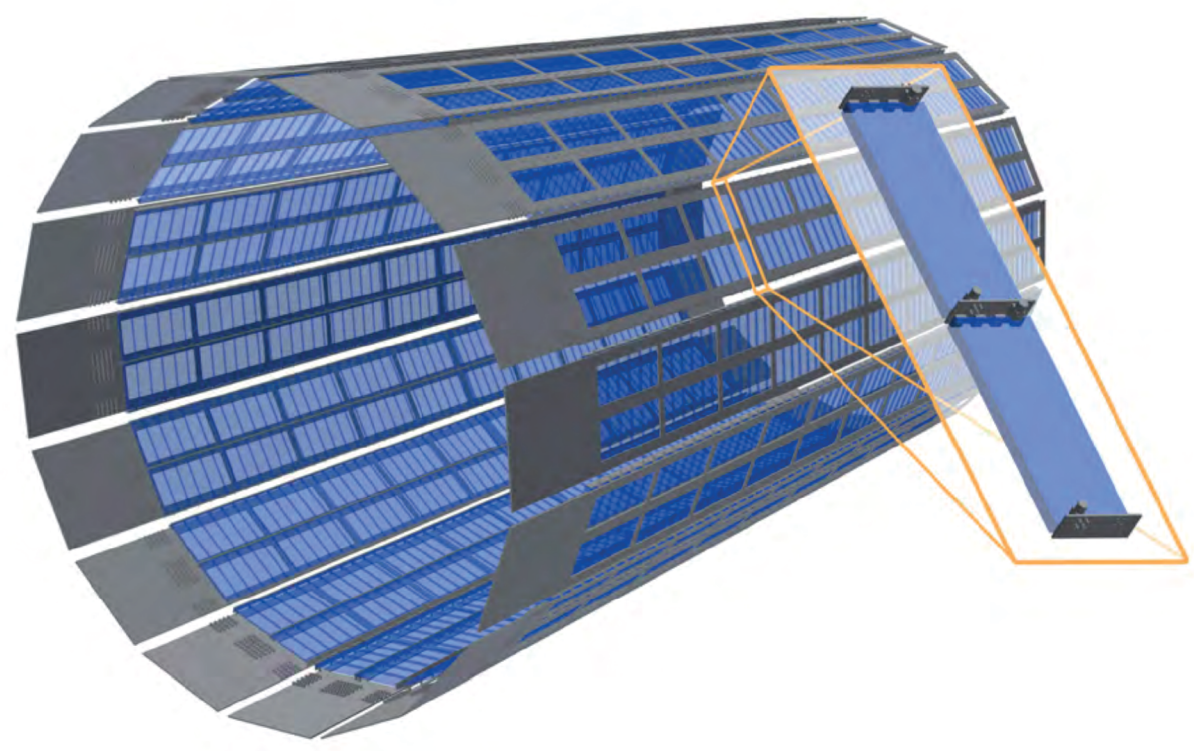

Figure 2.11: The Barrel Time-of-Flight detector layout. A pair of scintillator tiles in a super-module is highlighted [17].

tection layers and passive iron layers. The detection layers will be fabricated using Mini Drift Tubes (MDT). They consist of gold-plated tungsten anode wires inside a rectangular aluminum tube. The barrel part will combine 12 sensitive detection layers alternating with iron yoke layers; each layer is $3 \mathrm{~cm}$ thick. The barrel part is designed to enclose the TS and will be constructed from eight wheels with an octagon-shaped cylinder with a total of 2133 MDTs. The forward end-cap combines 6 detection layers alternating with 5 absorbing iron yoke absorbers. In total, 618 MDTs will be installed. The muon filter has almost the same structure as the forward end-cap. Five sensitive layers enclosing four iron absorber layers will be placed in the gap between the solenoid and the dipole magnet, utilized to enhance the depth of the absorber and serves to perform a magnetic screening between the solenoid and dipole magnetic field. The muon filter consists of 424 MDTs. A full description of the Muon System can be found in [18].

\subsubsection{Forward Spectrometer}

The Forward Spectrometer (see Fig. 2.13 ) will detect particles that are emitted with polar angles below $10^{\circ}$. The FS will have the same functionality as the TS with respect to tracking, PID, calorimetry, and muon detection. Hence, most FS sub-systems are based on the same detection principles as their TS equivalents. The Forward Tracking System [19] is comprised of six stations and a dipole magnet [11] and will be used to perform track reconstruction in the FS. The predicted spatial resolution is $\sigma=0.1 \mathrm{~mm}$ per detection station, resulting in better than 1 percent momentum resolution. Particle identification in the FS is carried out using the Forward Time-of-Flight detector [20] and Forward RICH counter. The key element of the RICH detector is a thin Aeorogel radiator for the generation of the Cherenkov radiation. The Cherenkov photons are then reflected by a planar mirror surface to an array of PMTs. The RICH is designed to perform 3 standard deviation pion $/$ kaon separation power for particle momenta up to $10 \mathrm{GeV} / \mathrm{c}$. The Forward Spectrom- 

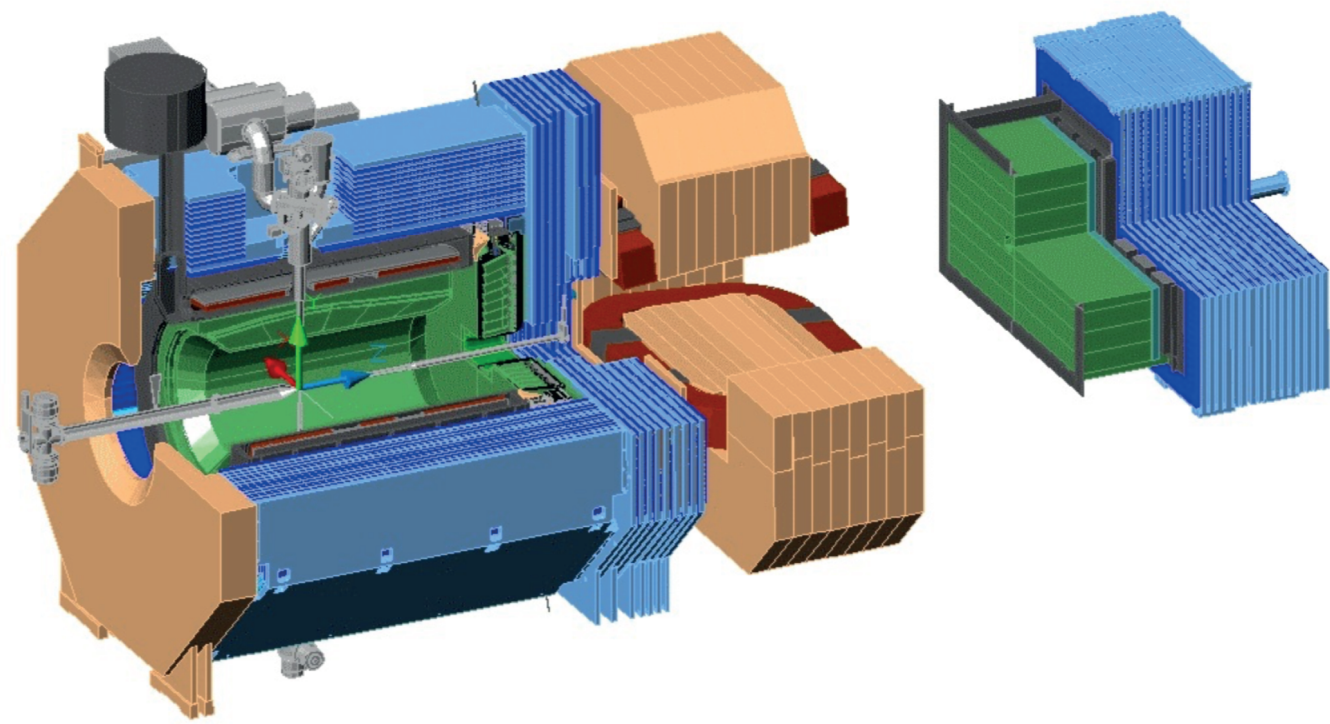

Figure 2.12: Muon system of PANDA. The Muon systems are highlighted in blue and the calorimeters in green [18].

eter Calorimeter [21] is located downstream from the RICH detector. The Muon Range System is positioned downstream of the forward-spectrometer calorimeter to reconstruct muon tracks. Lastly, the PANDA luminosity detector [22] uses elastic scattering of antiprotons on protons to determine the interaction rate by measuring antiprotons deflected at low angles [4] and will be installed downstream at the end of the spectrometer.

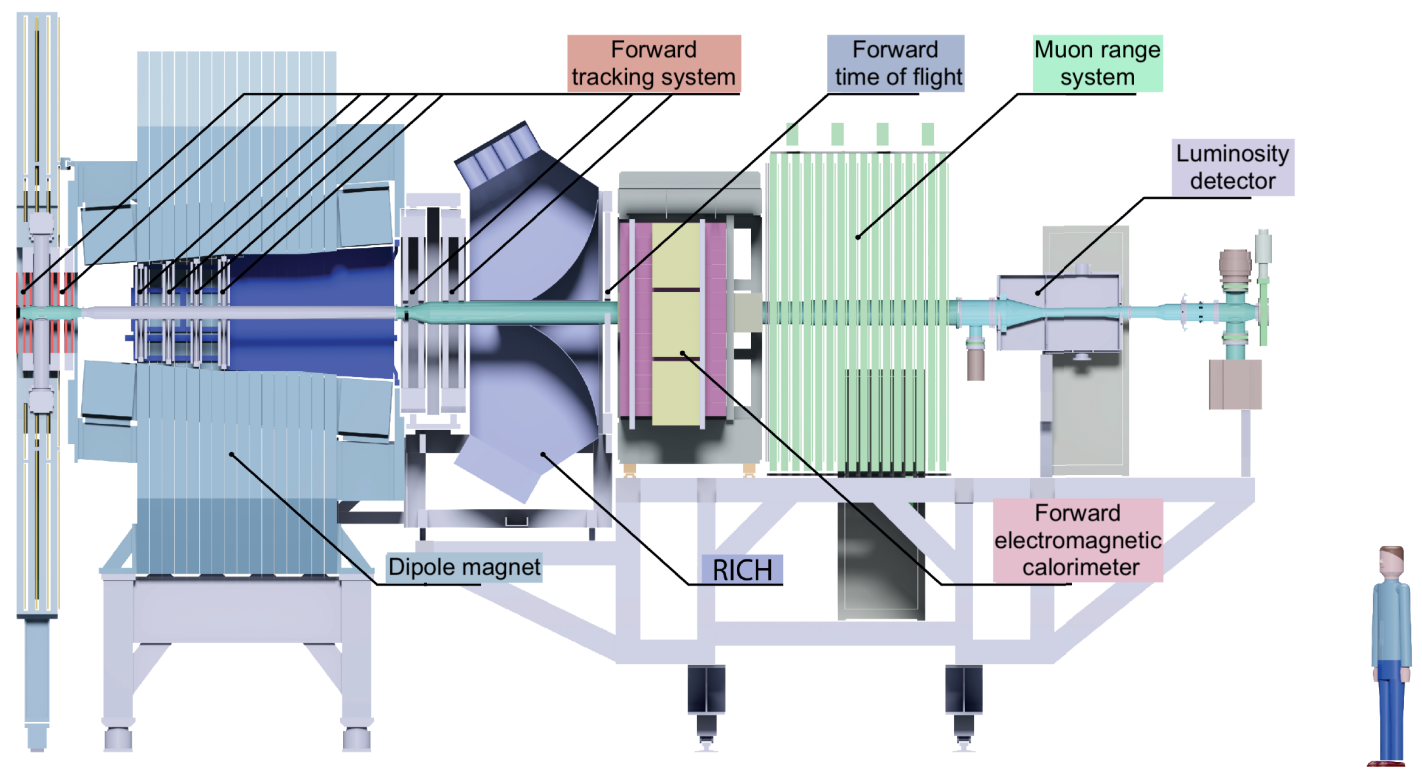

Figure 2.13: The PANDA Forward Spectrometer, the antiproton beam enters from the left [5]. 


\section{Chapter 3}

\section{The PANDA Barrel DIRC}

The main goal of the design of a Barrel DIRC detector for the PANDA experiment is to deliver clean pion and kaon separation for momenta up to $3.5 \mathrm{GeV} / \mathrm{c}$. The design is inspired by the successful BABAR DIRC. Several improvements were introduced to match the smaller PANDA experiment, and to reduce the production cost.

\subsection{DIRC Principle}

A charged particle, passing through a radiator with a refractive index $n$ with a velocity $v$ larger than the speed of light in that medium $(v>c / n)$, emits Cherenkov radiation. The Cherenkov photons are emitted on a cone surface with a half opening angle of $\cos \theta_{C}=1 / \beta n(\lambda)$. Due to dispersion in the medium, the refractive index and the Cherenkov angle $\theta_{C}$ depend on the wavelength of the emitted photons. The DIRC principle is the combination of generating Cherenkov photons and transporting them to the photon counters by internal reflection (Fig. 3.1). In other words, the radiator acts as a light guide. Therefore, the radiator for a DIRC counter is made of a highly-polished synthetic fused silica.

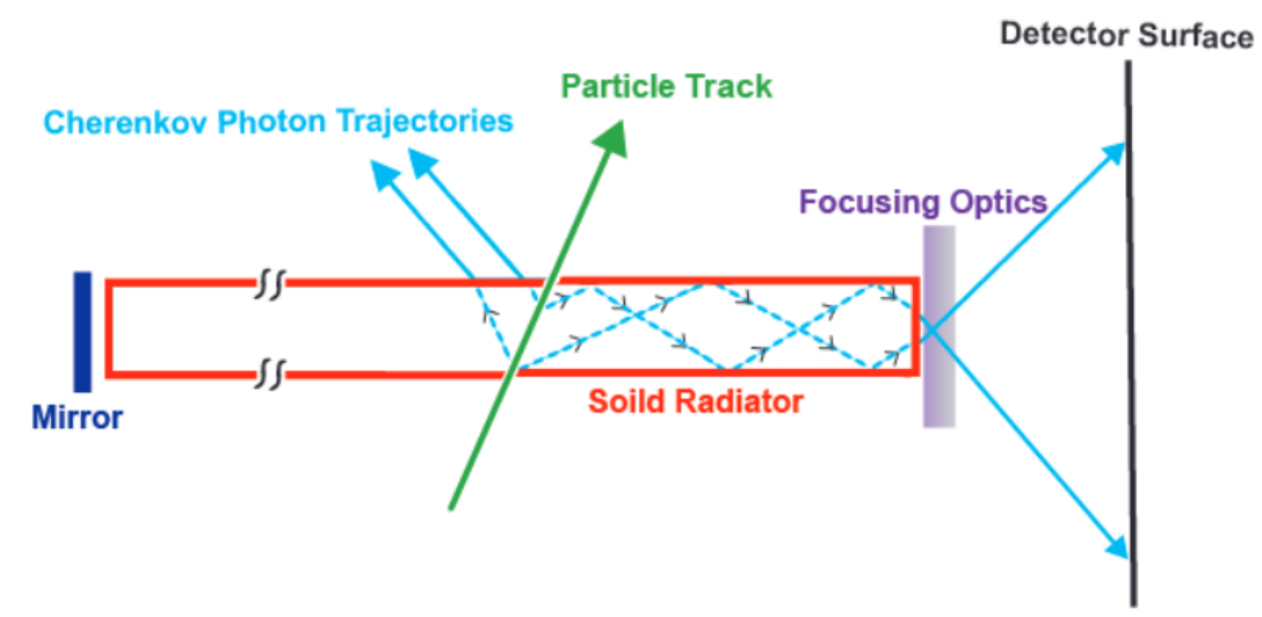

Figure 3.1: Schematic of the basic DIRC principle.

Fig. 3.2 shows the average Cherenkov angle for synthetic fused silica at $n=1.473$ at $380 \mathrm{~nm}$ as a function of the particle momentum. For a charged particle passing 
through this material exceeding the light velocity $(\beta \approx 1)$, some of the generated Cherenkov photons will be trapped inside the bar due to total internal reflection causing the produced photons to propagate towards the bar ends.

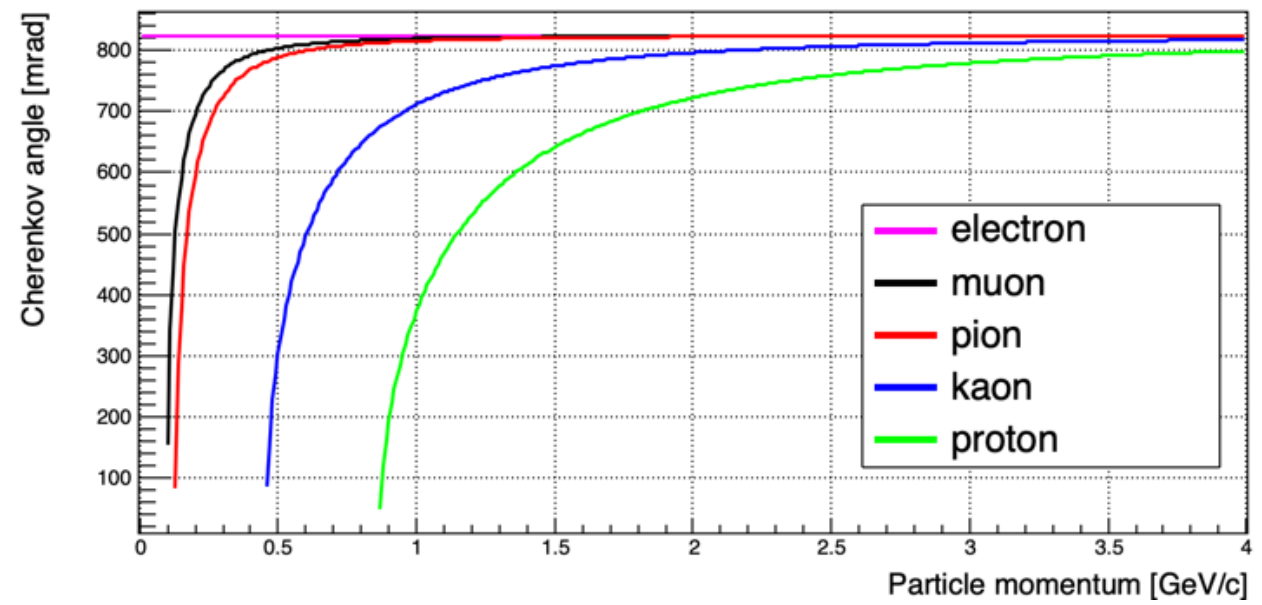

Figure 3.2: Cherenkov angle as function of the particle momentum for charged particles in synthetic fused silica.

A mirror is placed at the forward end of the bar to redirect the photons to the other end (readout). If the photons propagate through a highly polished bar with a rectangular cross-section, the magnitude of the Cherenkov angle will be conserved during the reflections inside the bar. The photons exit the bar through a focusing lens into the expansion volume (EV) towards the detection surface. The Cherenkov ring expands in the EV until it reaches the photo-detector surface, providing time and position information. A lookup table (LUT) can be used to transform this information into a direction measurement at the end of the bar. The Cherenkov angle can be reconstructed by combining the photon propagation time and direction with the particle momentum measurements obtained from the tracking system and the corresponding PID hypothesis likelihoods can be determined.

\subsection{PANDA PID Requirements}

The rich scientific physics program of the PANDA experiment requires a wide phase space coverage with particle identification systems. Although a fixed target experiment favors producing tracks with relatively low transverse momentum, pointing preferably towards the forward region, many particles are emitted into the barrel region of the target spectrometer, in the polar angle range between $22^{\circ}$ and $140^{\circ}$. The fraction of kaons of different benchmark reactions [15] (e.g., from charmonium and open charm decays, predominantly proceed via strangeness production from weak decays of the charm quarks), going into the barrel part of the TS is illustrated in Fig. 3.3. The phase space region of the PANDA Barrel DIRC defined is marked with a dashed rectangle. 


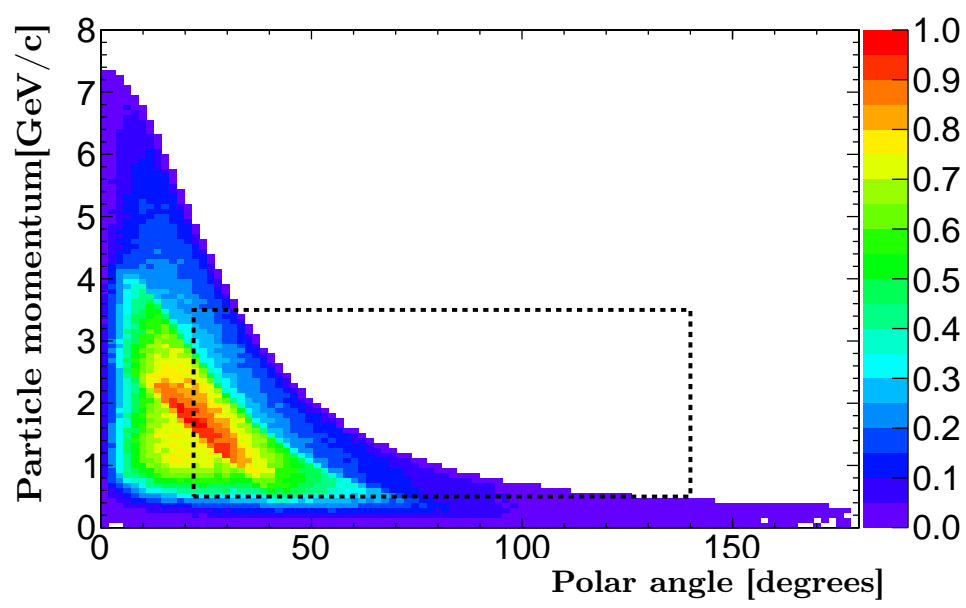

Figure 3.3: Phase space distribution of kaons for $p_{\bar{p}}=7 \mathrm{GeV} / c$ for benchmark physics channels. The Barrel DIRC coverage region is marked with the dashed rectangle [15].

Given that pions are the most abundant charged hadrons produced in $\bar{p} p$ annihilations, PID of the charged hadrons in the TS has to be able to cleanly separate pions from kaons for momenta up to $3.5 \mathrm{GeV} / c$. The figure of merit from this perspective is chosen to be the separation power $N_{\text {sep }}$. The separation power for Gaussian likelihood distributions can be defined as the absolute value of the difference of the two mean values $\left(\mu_{1}, \mu_{2}\right)$ in units of the average of the two standard deviations $\left(\sigma_{1}, \sigma_{2}\right)$ :

$$
N_{\text {sep }}=\frac{\left|\mu_{1}-\mu_{2}\right|}{0.5\left(\sigma_{1}+\sigma_{2}\right)}
$$

To provide a clear kaon identification, the pion/kaon separation power $\left(N_{\text {sep }}\right)$ must be at least 3 standard deviations (s.d.), over the entire phase space $22^{\circ}<\theta<140^{\circ}$ at $0.5 \mathrm{GeV} / c<p<3.5 \mathrm{GeV} / c$.

A RICH counter utilizing the DIRC principle [23] is an excellent choice to perform the PID in the barrel region of the Target Spectrometer. As the DIRC counter is thin compared to other alternative PID systems, both in radiation length and radius. This reduces the conversion of gammas measured by the EMC. It also keeps the outer detector components, the costly EMC and magnet, smaller. The fused silica bars are working both as Cherenkov radiators and as light guides. The photosensors and the readout electronics can be moved outside the compact active area of the central region of the PANDA Targeted Spectrometer. Current technology of the photo-sensors makes it possible to detect single photons even in the magnetic field of about $1 \mathrm{~T}$ and at average interaction rates of about $10-20 \mathrm{MHz}$ [15], this allows to operate the DIRC photon sensors inside the magnetic yoke. The first DIRC counter was used successfully in the BABAR experiment [24] where the $\pi / K$ separation power with more than 3 s.d. is achieved for momenta up to $4 \mathrm{GeV} / \mathrm{c}$. 


\subsection{PANDA Barrel DIRC Overview}

The PID performance of a DIRC counter is determined by the Cherenkov angle resolution $\sigma_{C}$, which can be calculated as

$$
\sigma_{C}^{2}=\sigma_{C, \gamma}^{2} / N_{\gamma}+\sigma_{\text {track }}^{2}
$$

where $N_{\gamma}$ is the number of detected photons and $\sigma_{C, \gamma}$ is the resolution of the Cherenkov angle measurement per photon (single photon resolution, SPR). The uncertainty of the track direction $\sigma_{\text {track }}$ is dominated by multiple scattering and the resolution of the PANDA tracking detectors. The PID reconstruction relies on the track information of the charged track. This track resolution is expected to be about $2 \mathrm{mrad}$ for high-momentum particles in the barrel region [15]. The SPR is defined by a number of contributions,

$$
\sigma_{C, \gamma}^{2}=\sigma_{\text {det }}^{2}+\sigma_{\text {bar }}^{2}+\sigma_{\text {trans }}^{2}+\sigma_{\text {chrom }}^{2}
$$

where $\sigma_{\text {det }}$ is the error due to the detector pixel size, $\sigma_{\text {bar }}$ is the contribution from optical aberration and imaging errors of the lens at the end of the bar, $\sigma_{\text {trans }}$ is the error due to bar imperfections, such as non-squareness, and $\sigma_{\text {chrom }}$ is the uncertainty in the photon production angle due to the chromatic dispersion $n(\lambda)$ of the fused silica material.

The predicted $\pi / \mathrm{K}$ separation power can be calculated for any given momentum as $\Delta\left(\theta_{C}\right) / \sigma_{C}$, where $\Delta\left(\theta_{C}\right)$ is the momentum-dependent difference of the expected Cherenkov angle (see Fig. 3.4) and the $\sigma_{C}$ is the Cherenkov angle resolution. For the momentum of $3.5 \mathrm{GeV} / \mathrm{c}$ the $\pi / \mathrm{K}$ angular difference is $\Delta\left(\theta_{C}\right)=8.5 \mathrm{mrad}$. Therefore, the design goal for the PANDA Barrel DIRC is $\sigma_{C}<2.8 \mathrm{mrad}$ [15].

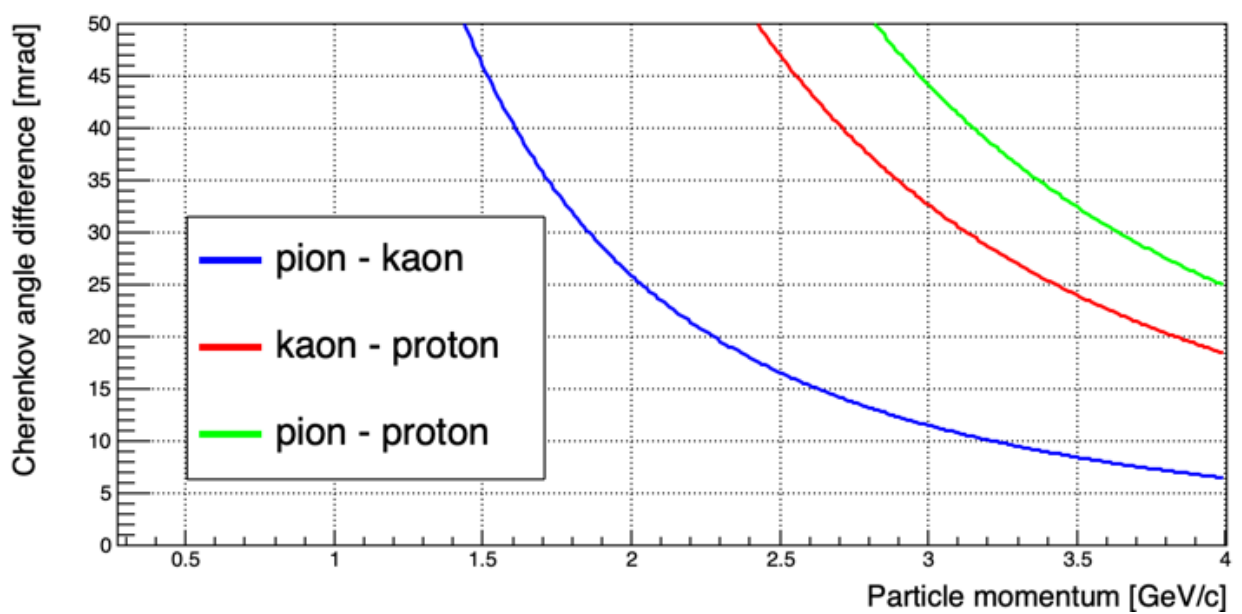

Figure 3.4: Cherenkov angle difference in synthetic fused silica for pions and kaons, kaons and protons, and for pions and protons.

The first DIRC counter was successfully operated at the BABAR experiment, where the DIRC concept was proven. Further advanced R\&D was performed for the SuperB FDIRC [25]. DIRC detectors are also used on the TOP counter of the Belle II 
experiment [26] and for the GlueX experiment [27] which is reuses parts of the DIRC from the BABAR experiment.

For the BABAR DIRC, the measured photon yield per particle in the range of $N_{\gamma}=15-60$, based on the charged track polar angle. A single photon Cherenkov angle resolution of $\sigma_{C, \gamma} \approx 10 \mathrm{mrad}$ is achieved. The track Cherenkov angle resolution is $\sigma_{C}=2.4 \mathrm{mrad}$, and clear $\pi / K$ separation of 3 s.d. at least for momenta up to 4 $\mathrm{GeV} / \mathrm{c}$.

\subsubsection{The PANDA Barrel DIRC Design}

Since the BABAR DIRC performance meets the PANDA PID requirements, the conservative approach was to follow the BABAR DIRC design when possible and to modify and optimize it for PANDA when necessary [15]. A schematic drawing of the PANDA Barrel DIRC detector is shown in Fig. 3.5. The polar angle range of $22^{\circ}$ to $240^{\circ}$ is covered by 16 optically isolated sectors, each comprising a bar box and a readout box, aligned around the beamline at a radius of $476 \mathrm{~mm}$.

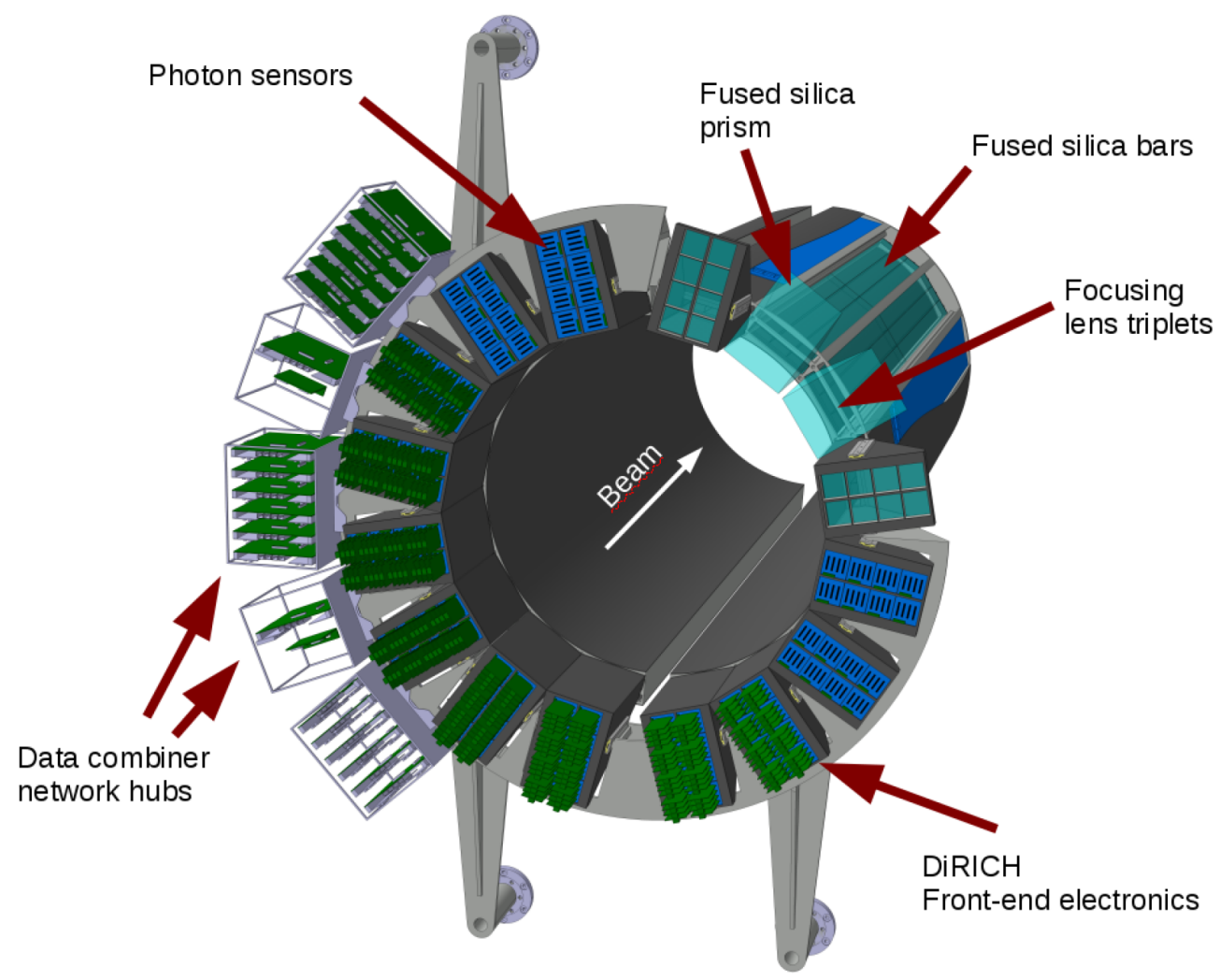

Figure 3.5: Schematic of the PANDA Barrel DIRC design [28].

Each bar box holds three bars of $17 \mathrm{~mm}$ thickness, $53 \mathrm{~mm}$ width, and $2400 \mathrm{~mm}$ length (two bars each $1200 \mathrm{~mm}$ are glued back-to-back using Epotek 301-2 [29]), located side-by-side in the bar box, separated by a small air gap. For each bar, at the forward end, a flat mirror is attached to reflect photons towards the readout end. The photons are focused by a complex 3-layer spherical compound lens composed of focusing and defocusing elements. The compound lens is used to match 
the focal plane of the back surface of the prism (detection plane). For each bar, the compound lens is glued with Epotek 301-2, which also serves as an exit window to the photons from the bar box. The bar box will be optically coupled to the prism. The coupling will be done using a silicone cookie (e.g., Momentive TSE3032 [30]). A $30 \mathrm{~cm}$-deep solid prism, made of synthetic fused silica, serving as expansion volume. The positions and arrival time of the photons are determined by an array of $2 \times$ 4 lifetime-enhanced Microchannel Plate Photomultiplier Tubes (MCP-PMTs) with a pixel pitch of $6.5 \mathrm{~mm}$ with a precision of about $100 \mathrm{ps}$. The photo-sensors and readout electronics are located in the region close to the backward end-cap of the solenoid where the magnetic field strength is $B \approx 1 \mathrm{~T}$ [15]. The bar boxes and the modular mechanical system components are made of Carbon-Fiber-Reinforced Polymer (CFRP). A cross-section of the CFRP structure and a bar box can be seen in Fig. 3.6. Additional CFRP containers are used to house the prisms and front-end cards so that each sector is combined into one light-tight unit and optically isolated from all other sectors. The CFRP containers are constantly flushed by boil-off dry nitrogen in order to remove moisture and residue from the outgassing of the bar box components. To facilitate access to the inner detectors of PANDA during the extended shutdown periods, the modular design permits the entire frame, which holds the prisms, sensors, and electronics, to be detached from the barrel structure that holds the bar boxes.

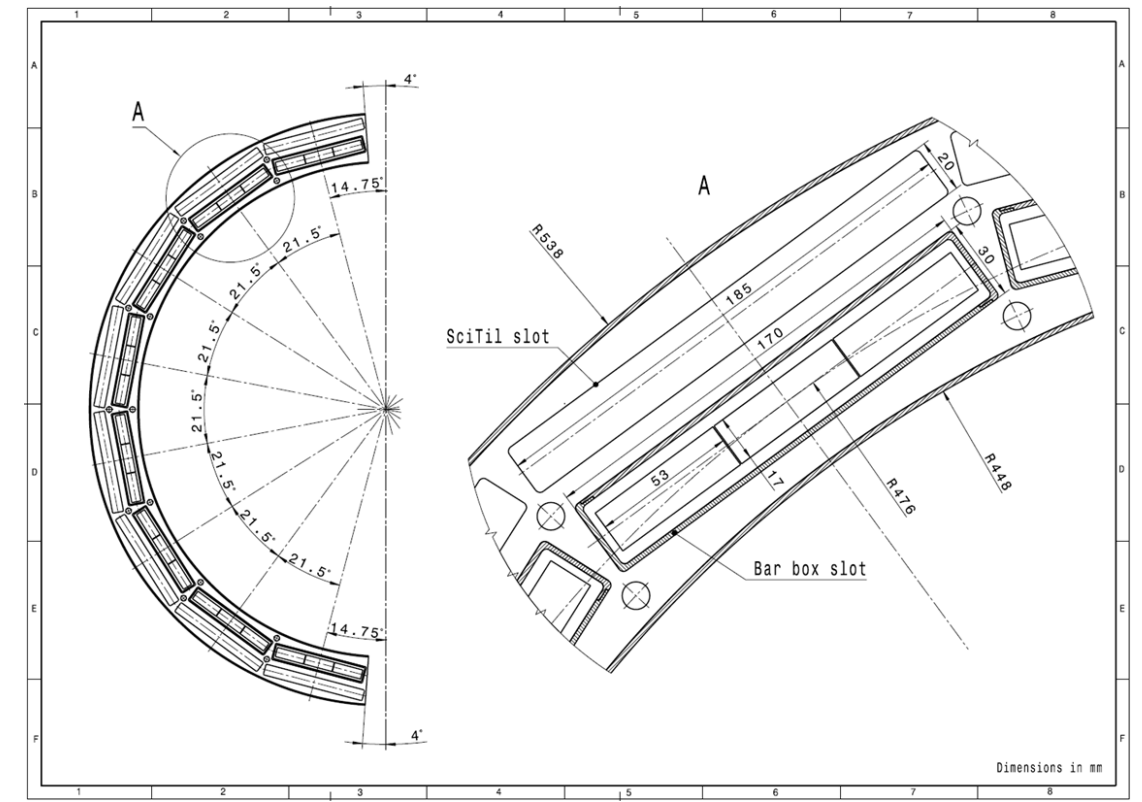

Figure 3.6: Central cross section view of the nominal Barrel DIRC geometry, including the space for the SciTil detector [15].

\subsection{PANDA Barrel DIRC Simulation and PID Per- formance}

The schematic diagram of the main optical components of the DIRC is shown in Fig.3.7. A detailed physical simulation of the PANDA Barrel DIRC was developed [31] in Geant4 [32]. The simulation includes the expected technical characteristics 
of the MCP-PMTs, lenses, optical coupling material, radiator and prism dimensions, and readout electronics to design the detector, optimize the performance, and to reduce the system cost. A full Geant simulation of the PANDA Barrel DIRC is illustrated in Fig. 3.8, a pion track (gray line) passing through a bar generates Cherenkov photons (orange lines), which are detected on a flat photo-sensor plane. The conic section of Cherenkov photons undergoes many internal reflections inside the bar and prism until being projected on the MCP-PMT plane, the accumulated histogram shows the unique hit pattern.

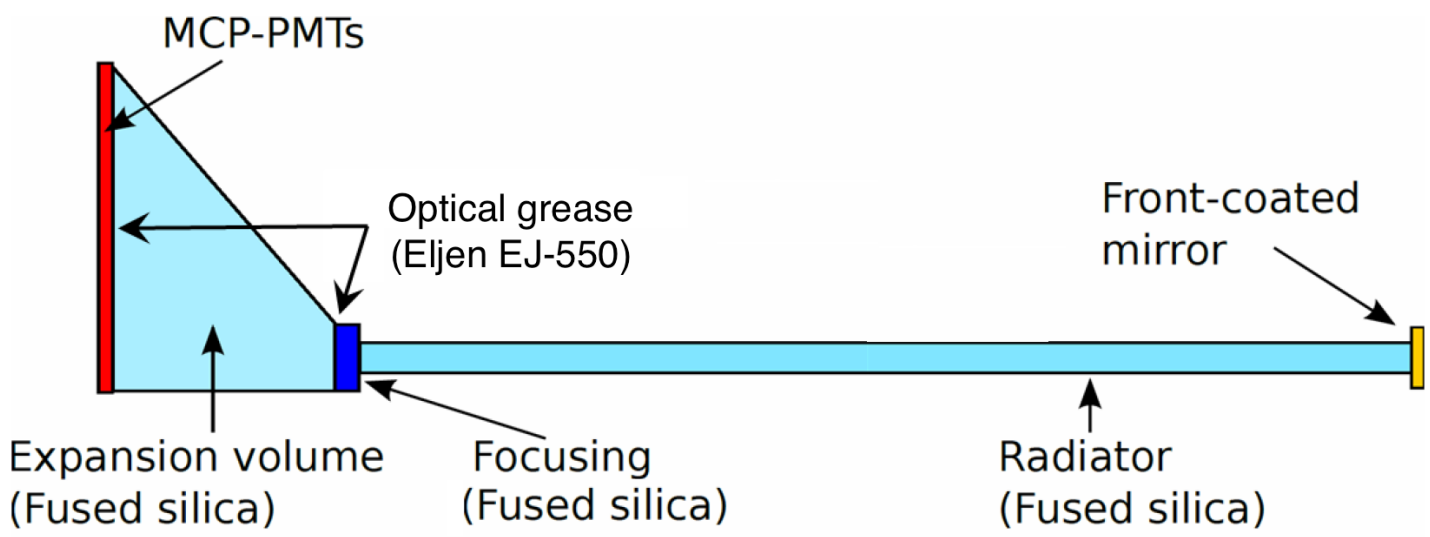

Figure 3.7: Simplified side view of the main optical components of the DIRC. Not to scale.

Several reconstruction algorithms were developed for the PANDA Barrel DIRC [33]. The "geometrical reconstruction" determines the Cherenkov angle by relying primarily on the position of the detected photons. The "time imaging", however, utilizes both position and time measurements by directly performing the maximum likelihood fit.

\subsubsection{Geometrical Reconstruction}

The geometrical reconstruction method is based on the successful BABAR DIRC [24] detector reconstruction approach. The method combines the known spatial position of the photon hit at the detection plane with the location and direction of the charged particle track in the bar and transforms them into a Cherenkov coordinate system. The detected photon direction is approximated by a $3 \mathrm{D}$ vector pointing from the end of the bar to the pixel, including effects of the refraction in the lens and reflections inside the prism, for every possible bar-pixel combination. Geant4 simulation was used to simulate optical photons at the end of the bar and to track them until they were detected on the photo-sensor plane. For each bar-pixel combination, the direction vectors were averaged and stored in a look-up table (LUT), see Fig. 3.9a. The Cherenkov angle $\theta_{c}$ is then calculated for each photon by combining the directions from the LUT with the charged particle direction vector, see Fig. 3.9b. Since the Cherenkov photons are internally reflected many times during propagation through the bar, the exact path of the photon is unknown, which 

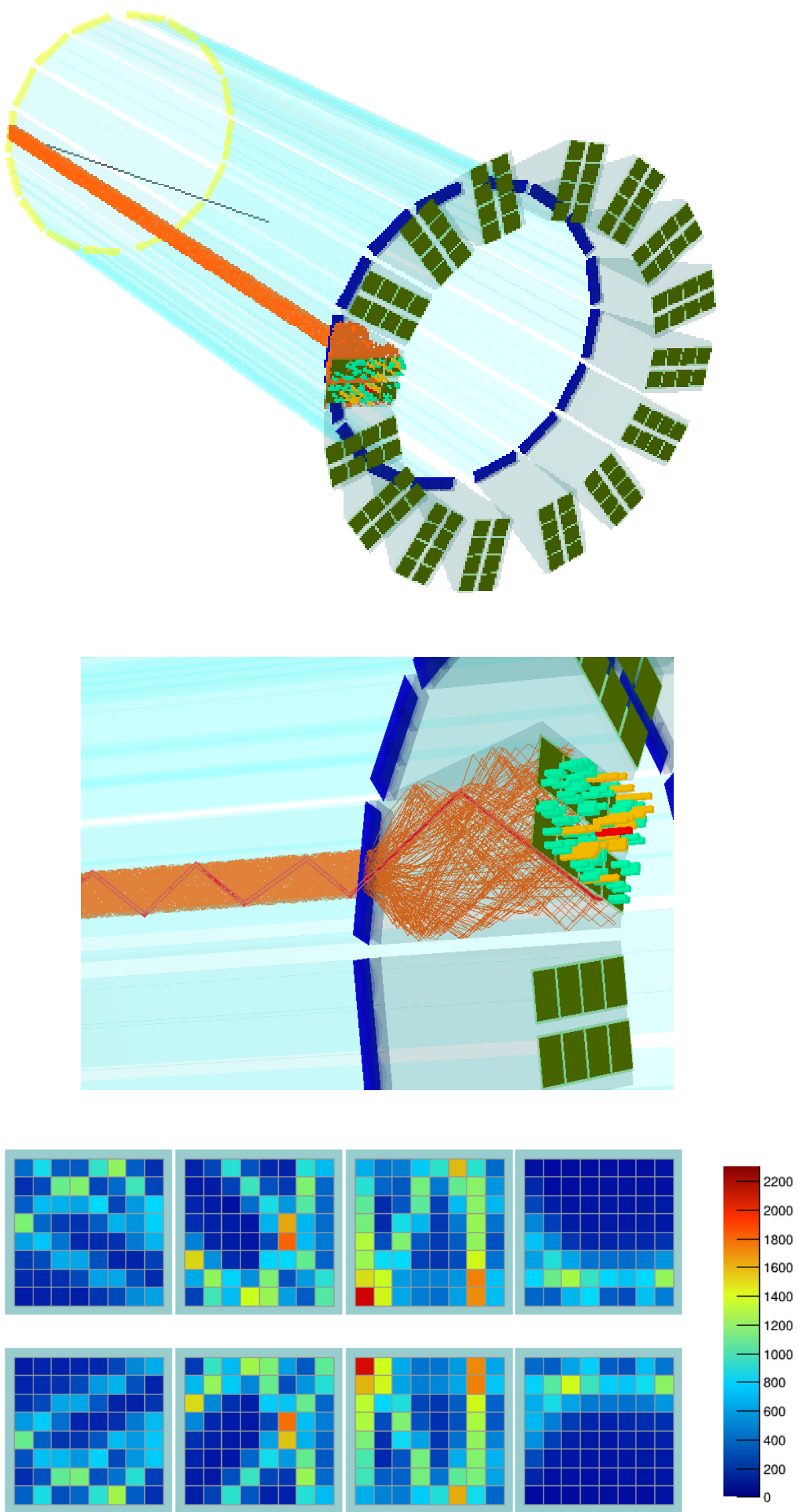

Figure 3.8: Geant simulation of the PANDA Barrel DIRC (top), close up (middle), and the colored histogram at the bottom shows the accumulated hit pattern from 1000 pions at $3.5 \mathrm{GeV} / \mathrm{c}$ momentum and $22^{\circ}$ polar angle (bottom). 
in turn leads to ambiguous reconstructed photon directions. There are eight different possible direction combinations inside the bar (right/left, top/bottom, and forward/backward). They are taken into account by combining the direction from the LUT in eight different ways with the particle direction, leading to up to eight values for the reconstructed Cherenkov angle per photon. The forward/backward ambiguities are resolved by applying a cut on the propagation time inside the bar. Further reconstruction ambiguities arise from the different possible reflections inside the prism, leading to 50 and more possible photon paths for some angles. The number of possible photon paths is reduced by discarding angles that are larger than the critical angle of fused silica (0.7476 rad) and by requiring the Cherenkov angle of the reconstructed photon to be smaller than $1 \mathrm{rad}$.
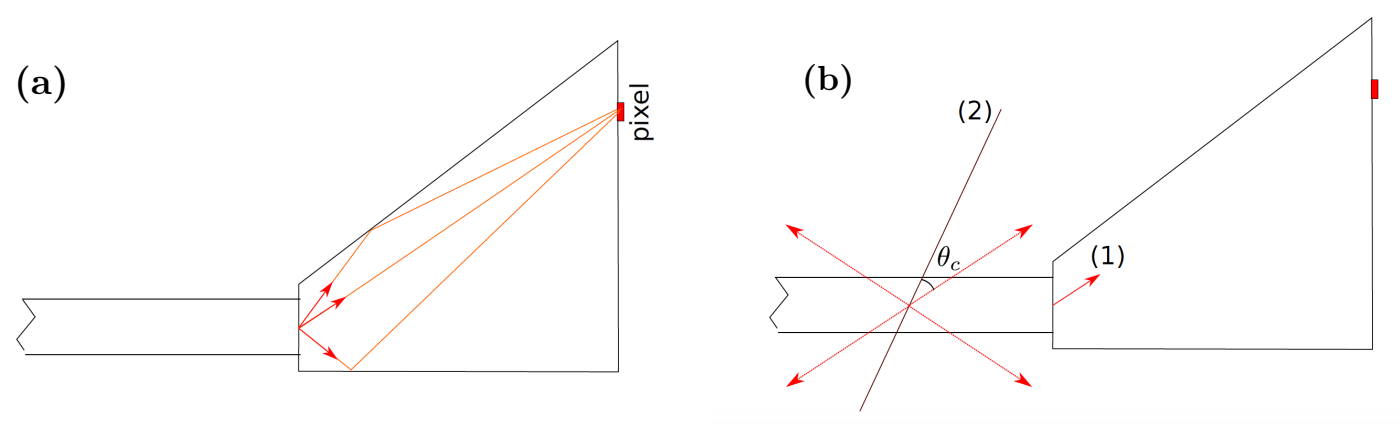

Figure 3.9: Schematic drawing of the geometrical reconstruction method. (a) All possible photon paths from the bar to the pixel (three examples are shown) are stored in look-up tables (LUT). (b) Close-up of the area of interest: The Cherenkov angle is determined by calculating the angle between the photon direction extracted from the LUT (1) and the charged track direction from the tracking system (2). Four of the eight possible ways to combine the photon vector with the track direction vector are shown [15].

Additional reduction of this combinatorial background can be achieved by applying a selection cut on the difference between the measured detection time of the photon and the expected detection time [15]. The latter is calculated from the reconstructed photon path in the bar and the prism, assuming a group velocity corresponding to a photon with the wavelength of $380 \mathrm{~nm}$. Figure 3.10a shows the time difference distribution for photons from 1000 pions with $3.5 \mathrm{GeV} / \mathrm{c}$ momentum at $22^{\circ}$ polar angle in Geant4. Figure $3.10 \mathrm{~b}$ shows a simulation of the reconstructed Cherenkov angle per photon, including reconstruction ambiguities, for 1000 pions with $3.5 \mathrm{GeV} / \mathrm{c}$ at $22^{\circ}$ polar angle. A clear peak can be observed at the expected value of the Cherenkov angle on top of the reconstruction ambiguities. In the last step, the distribution of Cherenkov angle per photon is fit with a Gaussian plus a linear background to calculate the likelihood for the distribution to originate from a e, $\mu, \pi, \mathrm{K}$, or $\mathrm{p}$ and to determine the mean Cherenkov angle for the track [15]. The main advantage of this reconstruction method is that it delivers a measurement of the SPR and the Cherenkov angle of the track, as well as the yield of signal and background photons, which are all important variables for the detector design. Furthermore, the algorithm is very fast since the LUTs depend only on the detector 
geometry and not on the particle properties, and thus can be created prior to event reconstruction [15].
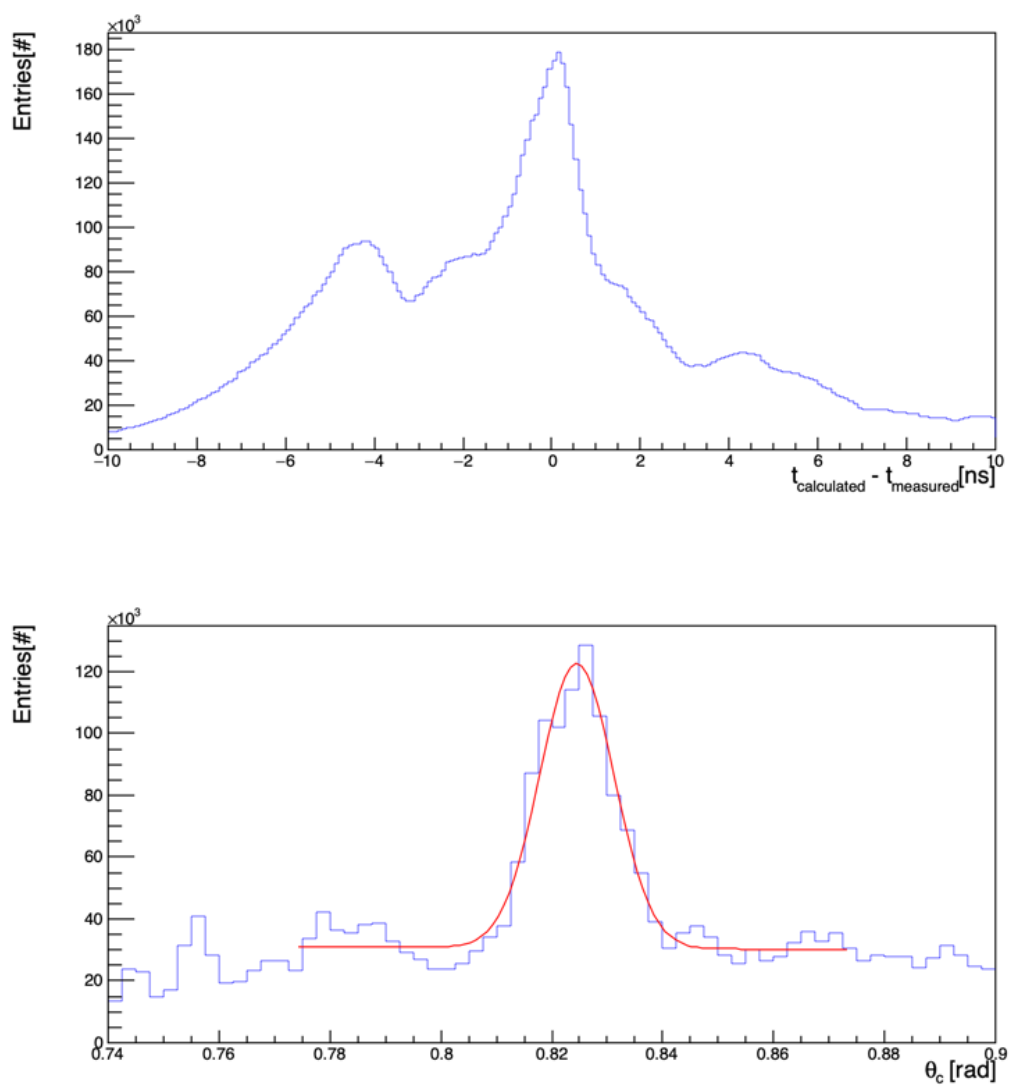

Figure 3.10: (a) Time difference between measured and expected arrival time of the Cherenkov photons from 1000 simulated pion with $3.5 \mathrm{GeV} / \mathrm{c}$ momentum at $22^{\circ}$ polar angle from reconstruction, (b) Simulation of the reconstructed Cherenkov angle per photon from 1000 pion fittied by Gassian pulse linear function. The fit result indicates that the Cherenkov angle resolution per photon is about $7 \mathrm{mrad}$.

The PID with the geometric reconstruction can be carried out by two ways. The first way is to extract the reconstructed Cherenkov angle for a charged particle and compare it to the expected values for the different hypotheses. Example for pions can be found in section 3.5.1. The second method is the direct likelihood hypotheses test used for the PANDA Barrel DIRC. For the PID using log-likelihood method, the signal measured by the DIRC is compared to the expected signals for different particle hypotheses. In case of the geometrical reconstruction, each of $N$ hit pixels has a set of $N_{a m b}$ reconstructed Cherenkov angles due to the ambiguities described above. The extended log-likelihood probability $\log \mathcal{L}$ for a given charged particle hypothesis $h(h=e, \mu, \pi, K, p)$ can be defined as [34]:

$$
\log \mathcal{L}_{h}=\sum_{i=1}^{N} \sum_{j=1}^{N_{i}^{a m b}} \log \left(S_{h}^{i j}+B_{h}^{i j}\right)+\log P_{N}\left(N_{e}\right),
$$

where $S_{h}^{i j}$ is the probability density function for different math hypothesis $h$, the signal and $B_{h}^{i j}$ is the background distribution. $N_{e}=N_{h}+N_{B}$ is the expected 
number of photons detected by the photo-sensors, the sum of the expected number of Cherenkov photons $N_{h}$ for hypothesis $h$ and the expected number of background photons, $N_{B}$. The last term in Eq. 3.4 is the Poisson probability to obtain $N$ photons if the mean is $N_{e}$.

The distribution of the log-likelihood differences of the pion/kaon hypothesis tests using Geant4 simulation with $3.5 \mathrm{GeV} / \mathrm{c}$ and $22^{\circ}$ polar angle is shown in Fig. 3.11.

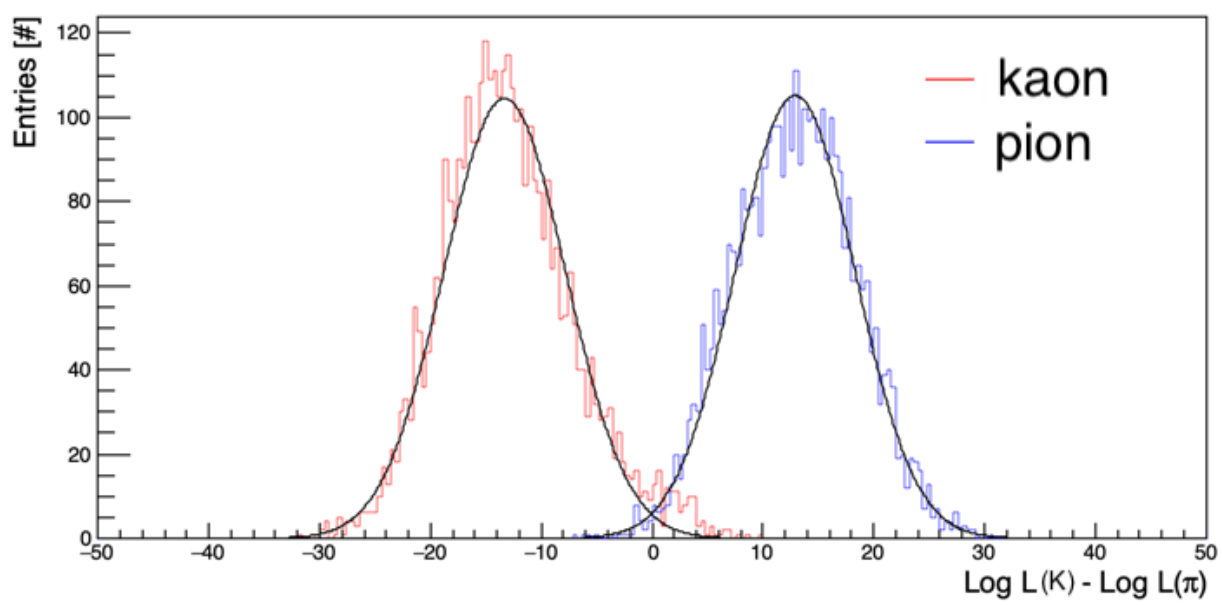

Figure 3.11: Example of $\pi / \mathrm{K}$ log-likelihood difference distributions for pions (red) and pions (blue) simulation events as result of the geometrical reconstruction at $3.5 \mathrm{GeV} / \mathrm{c}$ momentum and a $22^{\circ}$ polar angle. The $\pi / \mathrm{K}$ separation power value is $4.8 \pm 0.1$ standard deviation.

The distribution is fitted with two Gaussians and the $\pi / \mathrm{K}$ separation power is 4.8 s.d. as a result of geometrical reconstruction. The advantage of this reconstruction method is that it delivers measurements of the SPR, the Cherenkov angle of the charged particle track, and the photon yield, which are important for the detector performance evaluation. Moreover, the reconstruction algorithm is very fast since the LUTs can be created prior to event reconstruction and depend exclusively on the geometry of the detector and not on the charged particle properties.

\subsubsection{Time Imaging}

The time-based imaging method was first developed for the Belle II time-of-propagation (TOP) counter [26]. The basic idea is that the measured detection time of Cherenkov photons is compared to the expected photon detection time for every pixel and particle hypothesis combination, resulting in PID likelihoods. The expected photon detection times can be computed either analytically (for more details about analytical PDFs, see Ref. [35] ) or from full simulation, or taken from the beam data itself. The second approach was used to evaluate the PID of the time imaging approach for the PANDA Barrel DIRC. Figure 3.12 shows an example of the PDFs for kaons and pions using Geant 4 simulation at $3.5 \mathrm{GeV} / \mathrm{c}$ momentum and a polar angle of $22^{\circ}$ for two pixels in two different events. The detection time of the Cherenkov photons generated by kaons and pions is stored for every pixel and saved in an array of normalized histograms to create the probability density functions (PDF). 

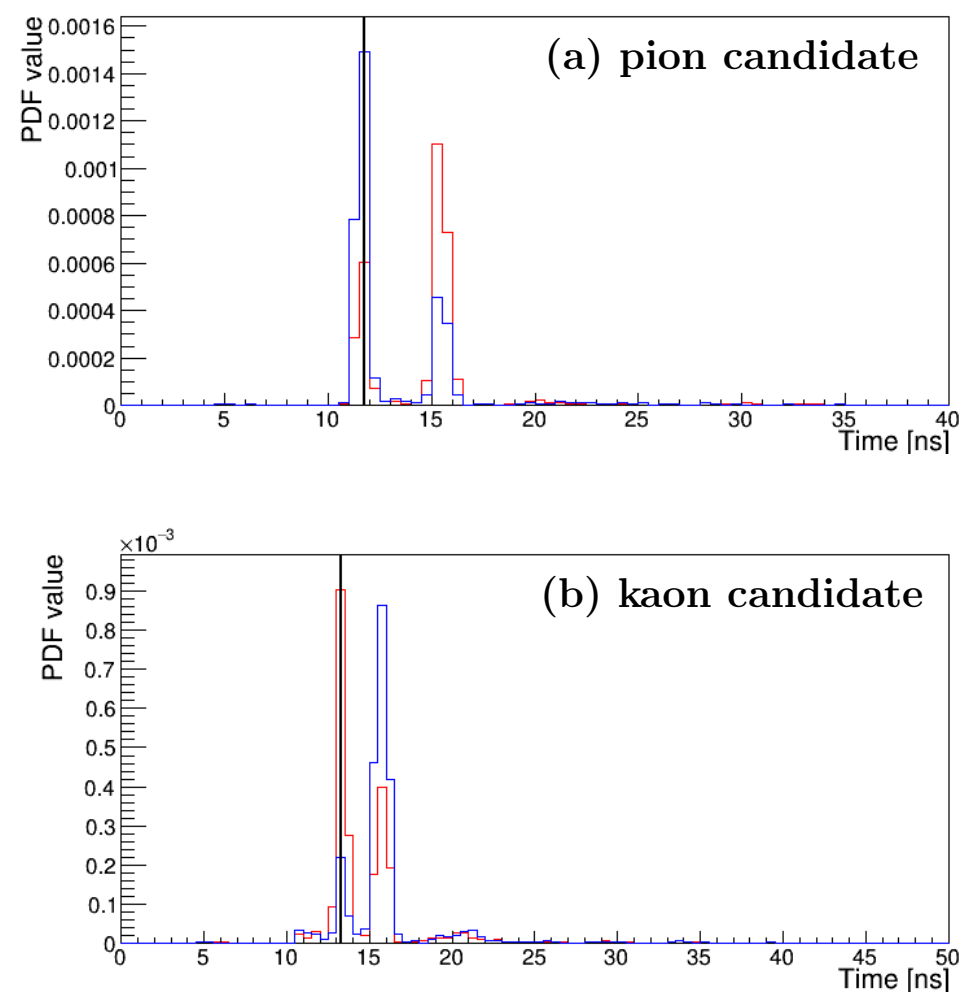

Figure 3.12: Example of the PDFs for two pixels for kaons (red) and pions (blue) determined from Geant4 simulation at $3.5 \mathrm{GeV} / \mathrm{c}$ momentum and a polar angle of $22^{\circ}$ for two pixels. The vertical lines indicate the observed hit times for a pion candidate (a) and a kaon candidate (b) 
The DIRC provides a measurements of the $N$ Cherenkov photons arrival times $t_{i}$ and positions $\left(x_{i}, y_{i}\right)$ on the photo-sensors plane. The extended log likelihood probability $\log \mathcal{L}$ for a given charged particle hypothesis $h(h=e, \mu, \pi, K, p)$ can be written as [34]:

$$
\log \mathcal{L}_{h}=\sum_{i=1}^{N} \log \left(S_{h}\left(x_{i}, y_{i}, t_{i}\right)+B_{h}\left(x_{i}, y_{i}, t_{i}\right)\right)+\log P_{N}\left(N_{e}\right),
$$

Where $S_{h}\left(x_{i}, y_{i}, t_{i}\right)$ is the signal time spectrum distribution at each pixel for the hypothesis $h$. $B\left(x_{i}, y_{i}, t_{i}\right)$ is the distribution for background, and $N_{e}=N_{h}+N_{B}$ is the expected number of detected photons, a sum of the expected number of signal photons $N_{h}$ for hypothesis $h$ and the expected number of background photons, $N_{B}$. The second term in Eq. 3.5 is the Poisson probability to obtain $N$ photons if the mean is $N_{e} . S_{h}\left(x_{i}, y_{i}, t_{i}\right)$ and $B\left(x_{i}, y_{i}, t_{i}\right)$ distributions normalization are carried out same way as described in Eq. 3.5.

The $S_{h}\left(x_{i}, y_{i}, t_{i}\right)$ and $B\left(x_{i}, y_{i}, t_{i}\right)$ distributions are normalized as follows:

$$
\begin{aligned}
& \sum_{j=1}^{n_{c h}} S_{h}\left(x_{j}, y_{j}, t\right) d t=N_{h} \cdot N_{e}, \\
& \sum_{j=1}^{n_{c h}} B\left(x_{j}, y_{j}, t\right) d t=N_{B} \cdot N_{e},
\end{aligned}
$$

Where the sum runs over all pixels $n_{c h}$ of the photon-sensors array, $\left(x_{j}, y_{j}\right)$ is the detector coordinates by which the photon is detected.

Figure 3.13 shows the log-likelihood difference for kaon and pion hypotheses for a sample $3.5 \mathrm{GeV} / \mathrm{c}$ of kaon and pions at $22^{\circ}$ polar angle. The calculated $\pi / \mathrm{K}$ separation power is 5.4 standard deviation.

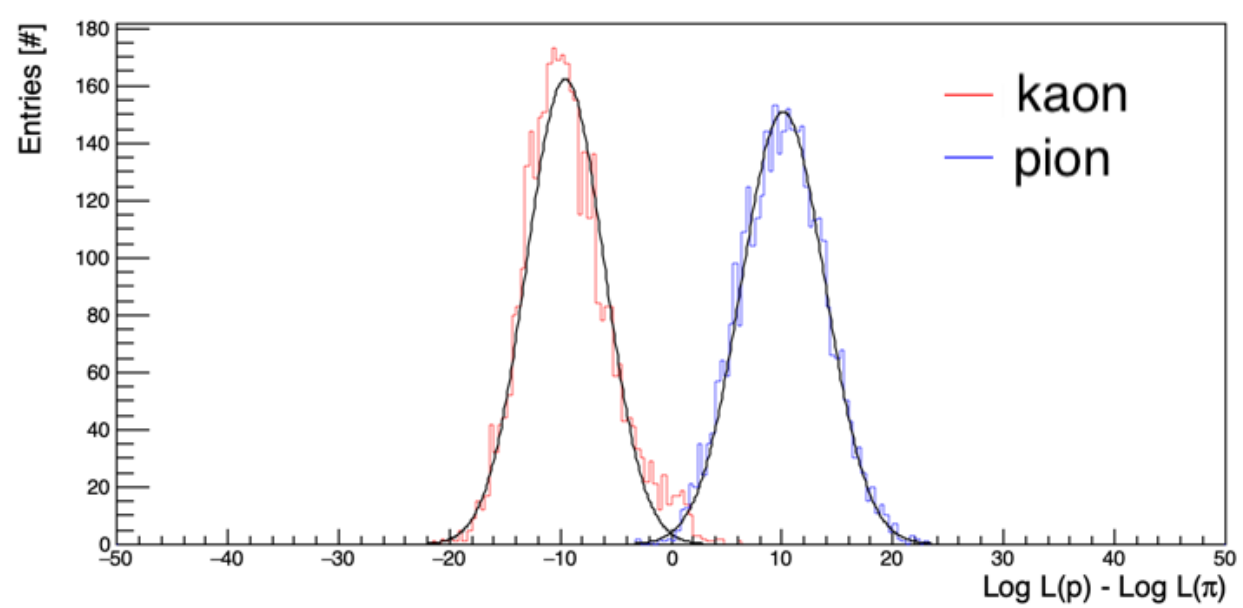

Figure 3.13: Example of $\pi / \mathrm{K}$ log-likelihood difference distributions for kaons (red) and pions (blue) simulation events as a result of the time-based imaging reconstruction at $3.5 \mathrm{GeV} / \mathrm{c}$ momentum and $22^{\circ}$ polar angle. The $\pi / \mathrm{K}$ separation power value is 5.4 standard deviation. 
The simulated $\pi / K$ separation power of the final design with three bars per bar box, 3-layer spherical lenses, and a prism with 8 MCP-PMTs is shown as a function of the particle momentum and polar angle in Fig. 3.14 using the time imaging PID algorithm. With a separation power of 4-14 s.d., the final design exceeds the PANDA PID requirement for the entire charged kaon phase space in PANDA.

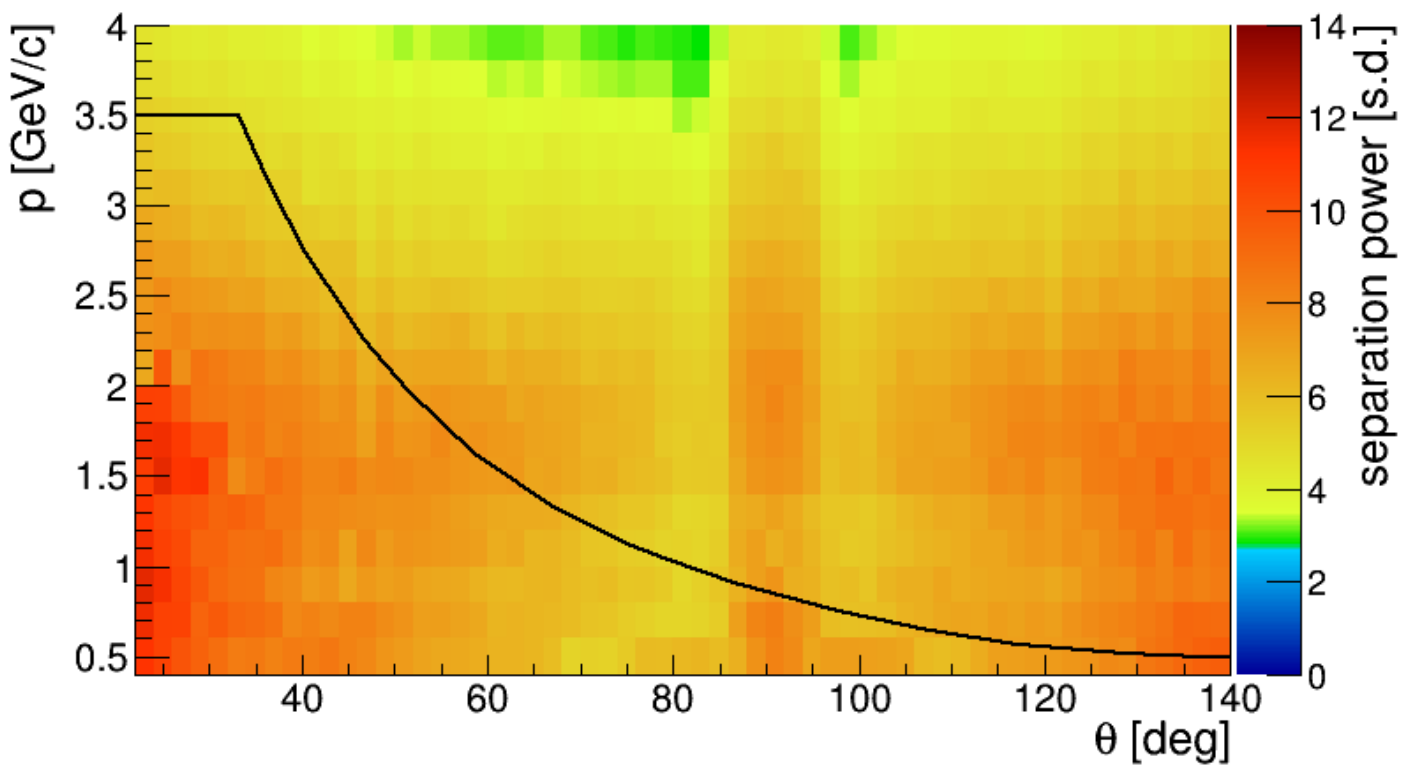

Figure 3.14: $\pi / K$ separation power as a function of particle momentum and polar angle in Geant simulation for the of the final design of the PANDA Barrel DIRC. The separation power was determined by the time-based imaging method. The area below the black line corresponds to the final-state phase space for charged kaons from various benchmark channels [15].

\subsection{The PANDA Barrel DIRC Prototype}

To validate the simulation and design ideas of the PANDA Barrel DIRC, several prototypes for the PANDA Barrel DIRC were developed to test the PID algorithms, optimize the performance, and to reduce the system cost. A detailed physical simulation of the PANDA Barrel DIRC prototypes in particle beams were developed [15] in Geant4 [32]. In the following section, the main aspects of the latest prototype (CERN 2018) simulation are discussed.

\subsubsection{Prototype Simulation Aspects}

A Geant4 [32] stand-alone simulation was developed for the beam test, incorporating many elements of the PANDA Barrel DIRC Geant4 simulation [31], utilizing the same physics processes, materials properties tables and beam properties at T9 [36].

The prototype simulation was an important element for the success of the beam tests at CERN (see Chapter 4). During the planning phase, the simulation was used to 
optimize the MCP-PMT layout on the prism focal plane and to choose the optimum locations of the optical diffusers for the laser calibration system. Furthermore, the simulation was performed for the data analysis phase to generate the look-up tables (LUT) for the geometrical reconstruction.

For each prototype configuration, the geometry of each optical component (e.g., the orientation of the bar relative to the lens and the prism) was adjusted to the values measured during periods of access to the prototype. Furthermore, the detailed specifications and characteristics of the Planacon MCP-PMTs [37] used during test beam campaign 2018 were implemented in the simulation. This included the experimentally observed dark count rate, charge-sharing, collection efficiency, and quantum efficiency as a function of the photon wavelength. The simulated single photon timing precision of the electronics was tuned to match the observed performance in the experiment. Figure 3.15a shows the event visualization of one simulated proton with $7 \mathrm{GeV} / \mathrm{c}$ momentum at $20^{\circ}$ polar angle with respect to the bar. Figure 3.15b shows a close-up of the simulated event visualization with Cherenkov photons paths.
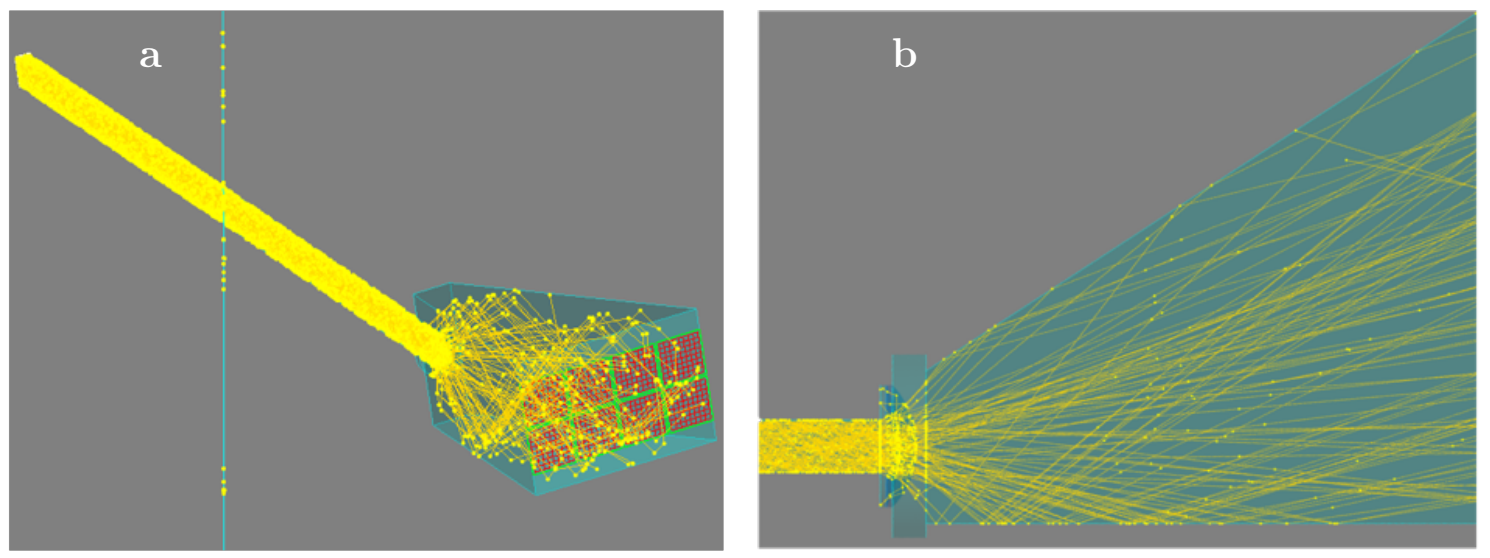

Figure 3.15: Example of the prototype Geant4 simulation: Visualization of protons at $7 \mathrm{GeV} / \mathrm{c}$ momentum at $20^{\circ}$ polar angle (a). Close-up of the region of the 3 -layer spherical lens in simulation (b), the yellow lines represent the Cherenkov photons.

\section{Expected Prototype PID Performance}

In the geometrical reconstruction, the single photon Cherenkov angle distribution (hits and reconstruction ambiguities) for each track is fitted with Gaussian plus linear background, the mean value is the Cherenkov angle of the track.

Figure 3.16 shows an example of the Cherenkov angle distribution per photon for a single proton track. The Cherenkov angle resolution per track $\sigma_{C, \text { track }}$ can be determined from the width of the difference between the measured, and the expected Cherenkov angles distribution. A similar approach was used in the BABAR DIRC, known as the track maximum likelihood fit [24]. Fig. 3.17 shows a track-by-track Cherenkov angle fit for simulated 2500 protons at $7 \mathrm{GeV} / \mathrm{c}$ momentum with $20^{\circ}$ 
polar angle.

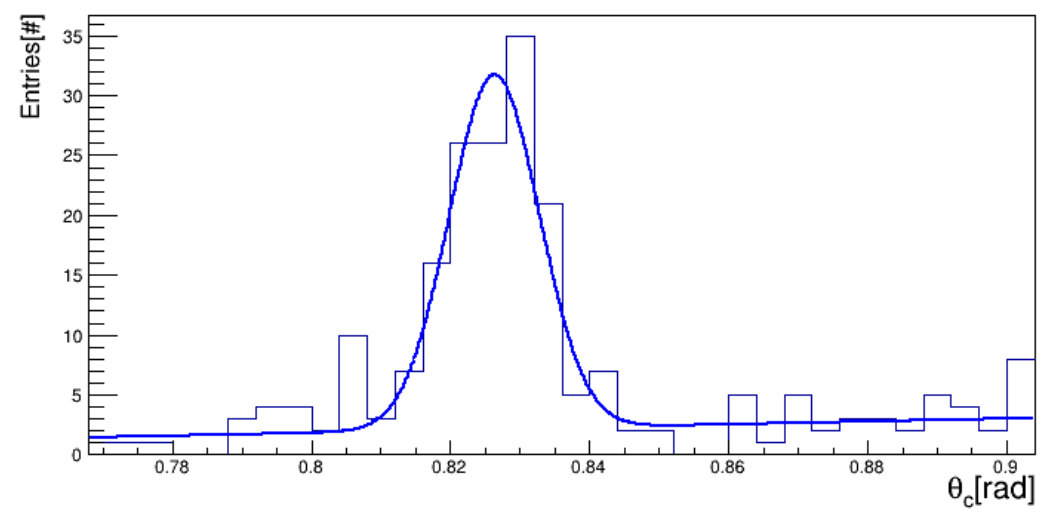

Figure 3.16: Single track Cherenkov angle per photon distribution for a simulated proton at $7 \mathrm{GeV} / \mathrm{c}$ moemtum with $20^{\circ}$ polar angle.

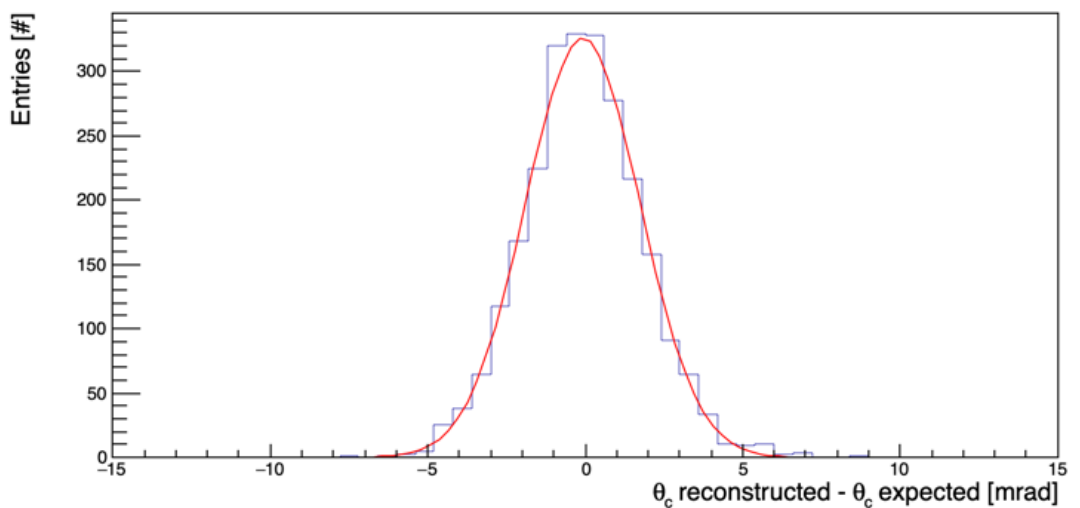

Figure 3.17: Resolution of the reconstructed Cherenkov angle per track for 2500 simulated proton at $7 \mathrm{GeV} / \mathrm{c}$ momentum with $20^{\circ}$ polar angle.

The distributions were fitted by a Gaussian function (red line) in the range of $\pm 5 \mathrm{mrad}$. The Cherenkov angle per track resolution $\sigma_{C, \text { track }}$ (the width of the fitting function) was found to be $1.8 \mathrm{mrad}$ for the $20^{\circ}$ polar angle. The $\pi / p$ separation at $7 \mathrm{GeV} / \mathrm{c}$ can be quantified as the Cherenkov angle difference of pions and protons at this momentum $\left(\Delta\left(\theta_{C}\right)=8.1 \mathrm{mrad}\right)$, divided by $\sigma_{C, \text { track }}$. This results in about 4.5 s.d. separation value at $20^{\circ}$.

Two main reconstruction algorithms were used to determine the detector figures of merit and to evaluate the performance of the near-final design of the PANDA Barrel DIRC. In the following section, the expected PID performance of the prototype is studied for the geometrical reconstruction and time-based imaging. 


\section{Geometrical Reconstruction}

In the geometrical reconstruction, most of the reconstructed photon directions are ambiguous. To reduce the combinatorial background, a cut on the time difference between the measured time of the Cherenkov photons (by the photo-sensors) and the calculated time (sum of the reconstructed photon propagation time inside the bar and prism) is performed. An example of the time difference distribution for proton beam data at $7 \mathrm{GeV} / \mathrm{c}$ momentum at $20^{\circ}$ polar angle is shown in Fig. 3.18a. The distribution shows a complicated structure that is not Gaussian-like peak as desired, due to the reconstruction ambiguities originated from the fact of existing several solutions for one single hit. To suppress the reconstruction ambiguities, a Cherenkov angle cut with $\pm 50 \mathrm{mrad}$ around the expected value of the Cherenkov angle is applied. Figure 3.18b illustrates the two-dimensional time selection cut, which is used to reduce the reconstruction ambiguities and to compensate the chromatic dispersion. The surviving photons after the time cut are used to reconstruct the Cherenkov angle and to perform particle hypothesis testing. The accumulated Cherenkov photon hit pattern on the MCP-PMTs after applying the time selection criteria is shown in Fig. 3.19.
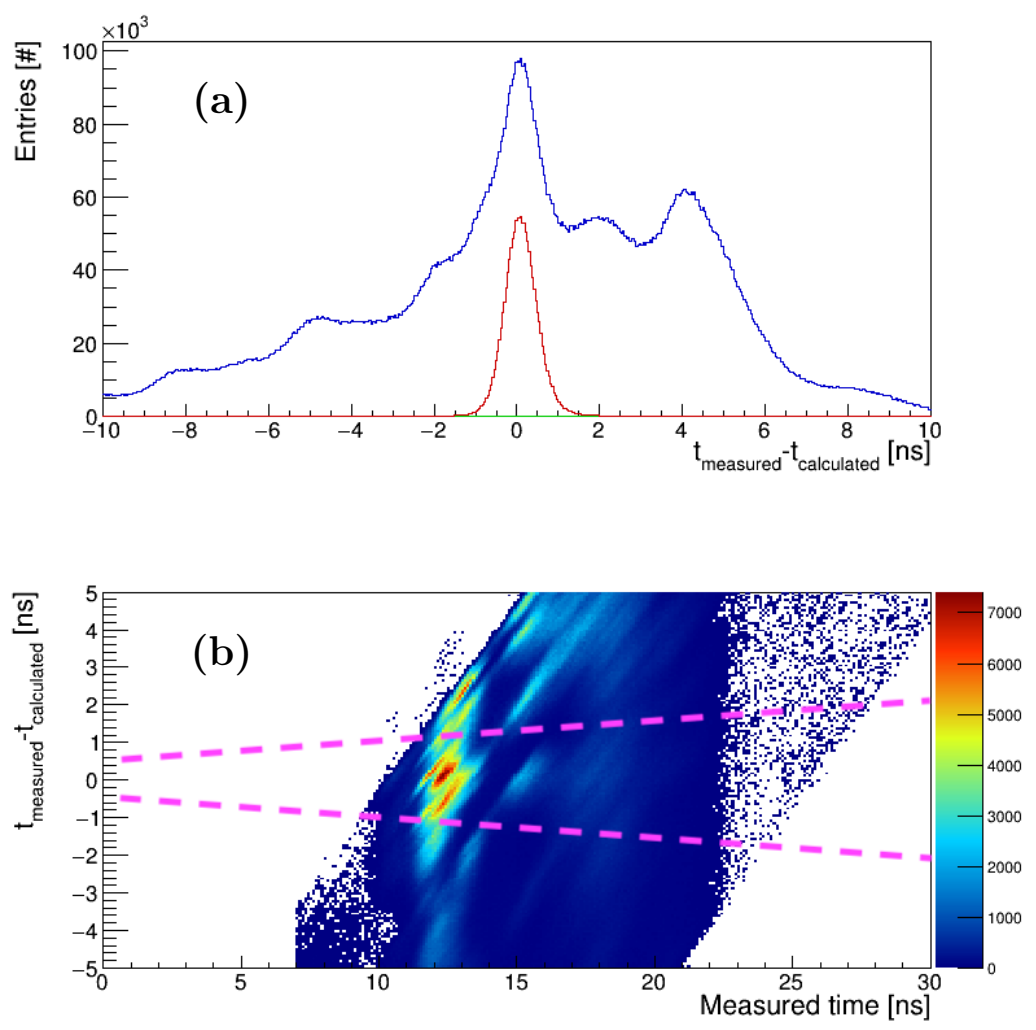

Figure 3.18: (a) Time difference distribution between measured and expected arrival time of the Cherenkov photons (blue) true path photons in the prism (red) after applying a Cherenkov angle cut, (b) A two-dimensions time difference cut.

Most of the reconstructed values of the photon angle are far from the correct one, forming a combinatorial background under the Cherenkov angle peak around the expected value. Figure 3.20 shows an example of the single photon Cherenkov angle 

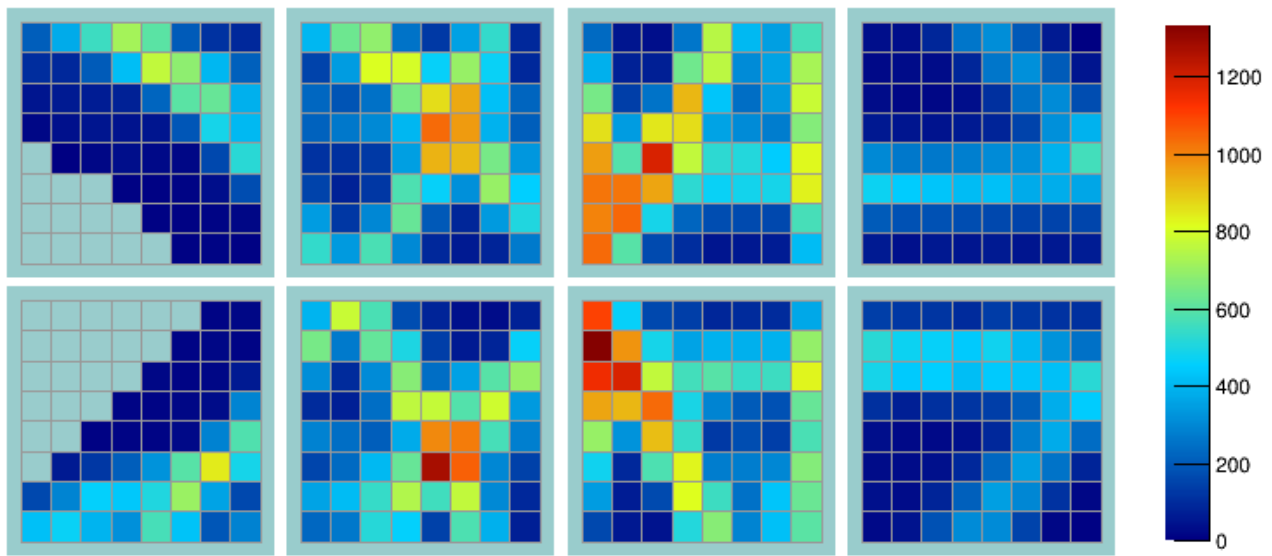

Figure 3.19: Accumulated hit pattern for 2500 simulated protons at $7 \mathrm{GeV} / \mathrm{c}$ momentum and $20^{\circ}$ polar angle.

distribution using Geant4 simulation for proton and pion samples at $7 \mathrm{GeV} / \mathrm{c}$ momentum at $20^{\circ}$ polar angle, the distributions were achieved after applying the time cut described above. The Cherenkov angle per photon distributions are fitted by a Gaussian plus linear background, the red and blue vertical lines show the expected value of the Cherenkov angle for proton and pion, respectively. The fit results indicate that the mean values of the fit match the expectation, with about $8.0 \mathrm{mrad}$ single photon Cherenkov angle resolution. Figure 3.21 shows the photon yield per track for simulated proton events as a result of the geometrical reconstruction at $7 \mathrm{GeV} / \mathrm{c}$ momentum and a $20^{\circ}$ polar angle. Each counted photon has at least one solution within the time selection criteria and its reconstructed Cherenkov angle has to be located within a window of $\pm 50 \mathrm{mrad}$ around the expected value of the Cherenkov angle.

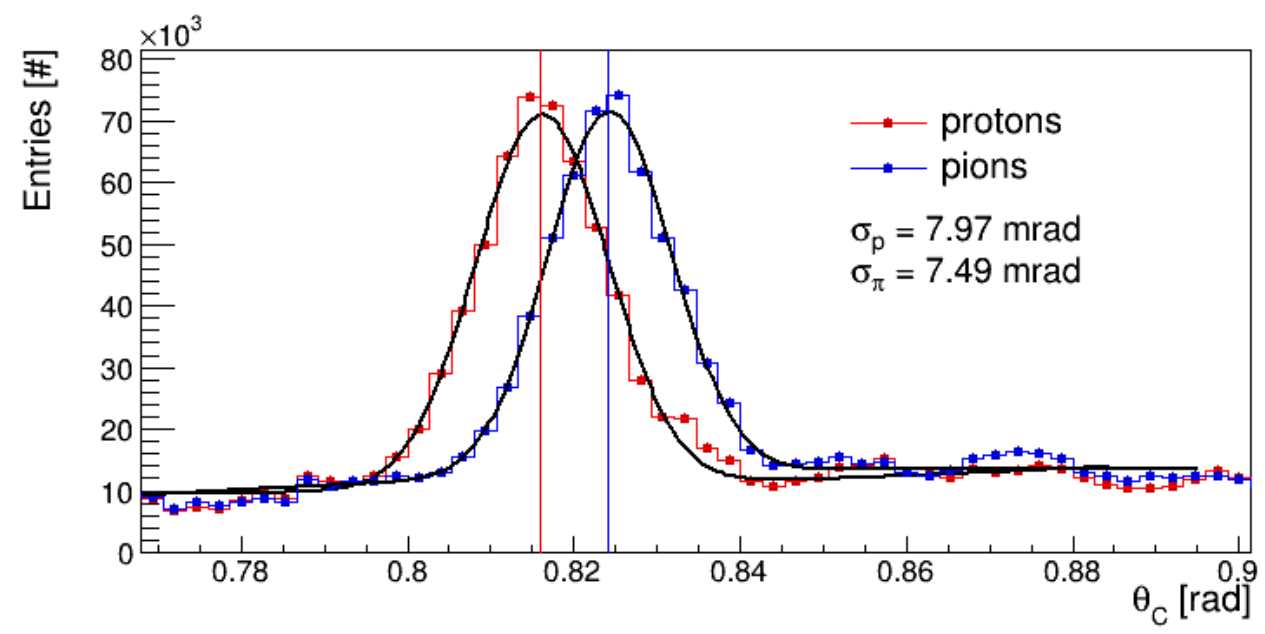

Figure 3.20: Examples of the reconstructed Cherenkov angle per photon from simulated protons and pions at $7 \mathrm{GeV} / \mathrm{c}$ momentum and $20^{\circ}$ polar angle.

The first geometrical approach is done as follows, for each event, the single photon Cherenkov angle distribution for all hits and ambiguities is compared to a Gaussian plus a linear background, where the mean value of the Gaussian is fixed to the 


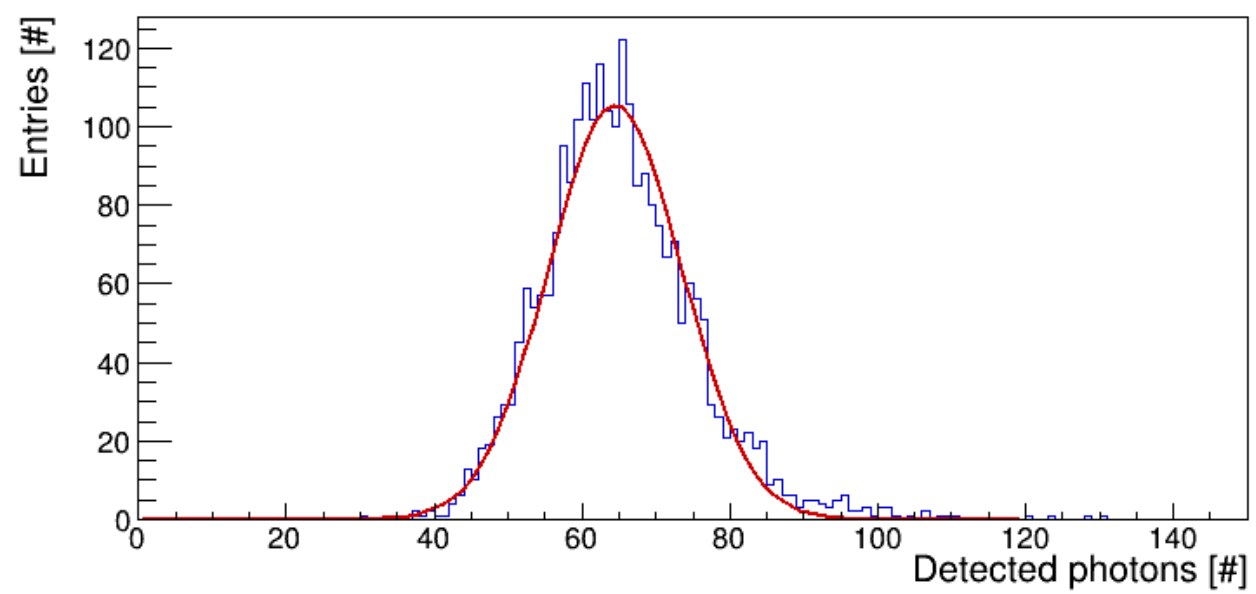

Figure 3.21: Example of the photon yield per track for 2500 protons from simulated sample at $7 \mathrm{GeV} / \mathrm{c}$ momentum and $20^{\circ}$ polar angle.

predicted Cherenkov angles for either pions or protons, and the Gaussian sigma is fixed to the expected SPR for that polar angle. The second approach of the geometrical reconstruction is similar to the first approach described above. However, the Cherenkov angle distribution for each track is fitted with Gaussian plus a linear background. The mean value of the fit is used to perform the particle hypothesis likelihood test. The log-likelihood differences distribution of the pion/proton hypothesis tests for simulated events with $7 \mathrm{GeV} / \mathrm{c}$ and $20^{\circ}$ polar angle for the first approach is shown in Fig. 3.22, and for the second approach is shown in Fig. 3.23. Both distributions are fitted with two Gaussians and the $\pi / \mathrm{p}$ separation power is $4.2 \pm 0.2$ standard deviation for each approach.

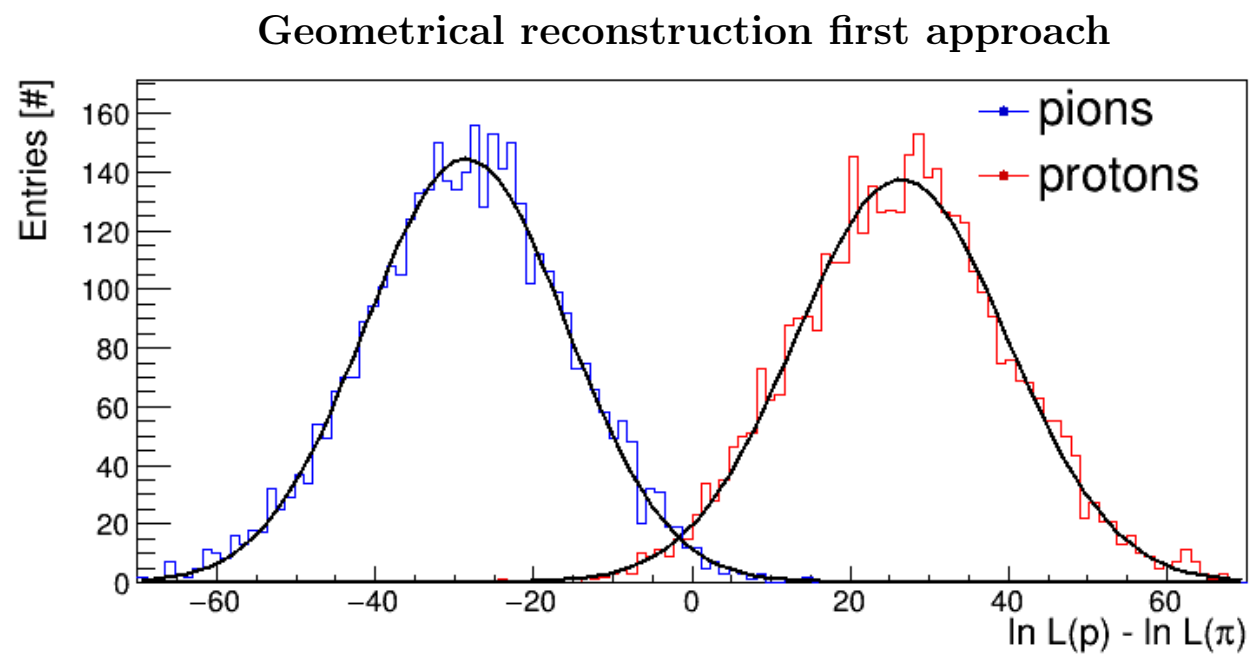

Figure 3.22: Example of the $\pi / \mathrm{p}$ log-likelihood difference distributions for 8000 simulated events of protosn (red) and pions (blue) as result of the geometrical reconstruction at $7 \mathrm{GeV} / \mathrm{c}$ momentum and a $20^{\circ}$ polar angle. The $\pi / \mathrm{p}$ separation power value is $4.2 \pm 0.2$ standard deviation. 


\section{Geometrical reconstruction second approach}

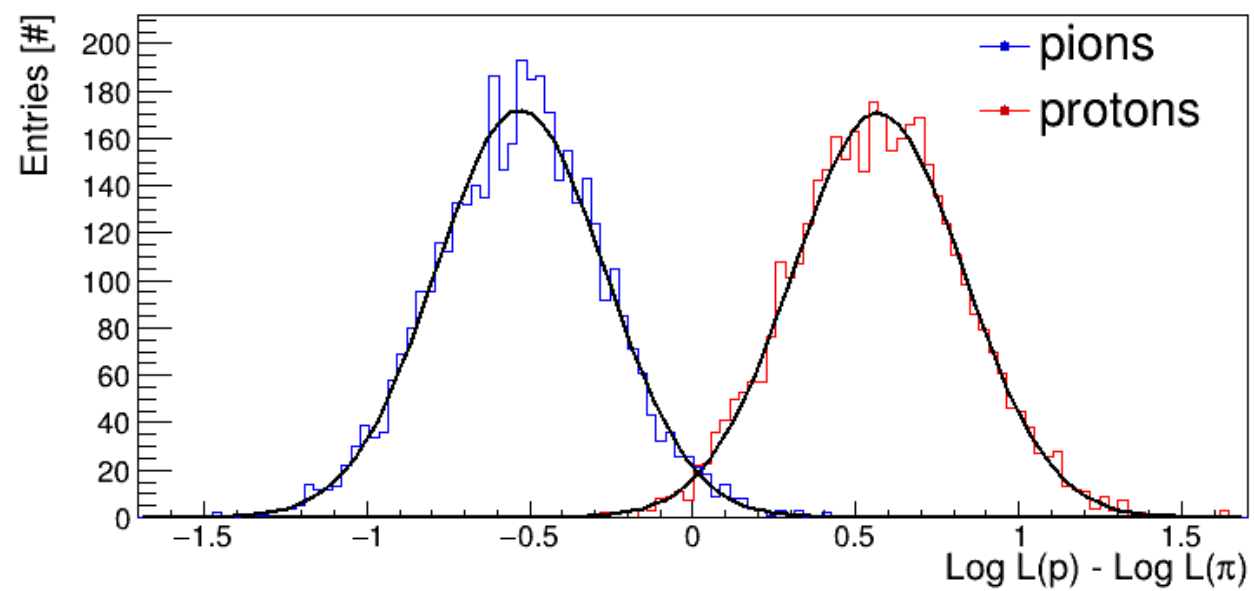

Figure 3.23: Example of the $\pi / \mathrm{p}$ log-likelihood difference distributions for 8000 simulated events of protosn (red) and pions (blue) as result of the geometrical reconstruction at $7 \mathrm{GeV} / \mathrm{c}$ momentum and a $20^{\circ}$ polar angle. The $\pi / \mathrm{p}$ separation power value is $4.2 \pm 0.2$ standard deviation.

\section{Time Imaging}

A detailed explanation of the time imaging PID method is described in section 3.4.2. The expected photon detection times can be computed either analytically or from full simulation. The latter approach was used to evaluate the expected PID performance of the time imaging approach for the PANDA Barrel DIRC prototype. A statistically independent simulation sample of 2000 pions and protons is used to create the simulation PDFs. Figure 3.24 shows the log-likelihood difference for proton and pion hypothesis for simulated events with $7 \mathrm{GeV} / \mathrm{c}$ protons and pions at $20^{\circ}$ polar angle. The calculated $\pi / \mathrm{p}$ separation power is $4.9 \pm 0.2$ standard deviations.

\section{Time-based imaging}

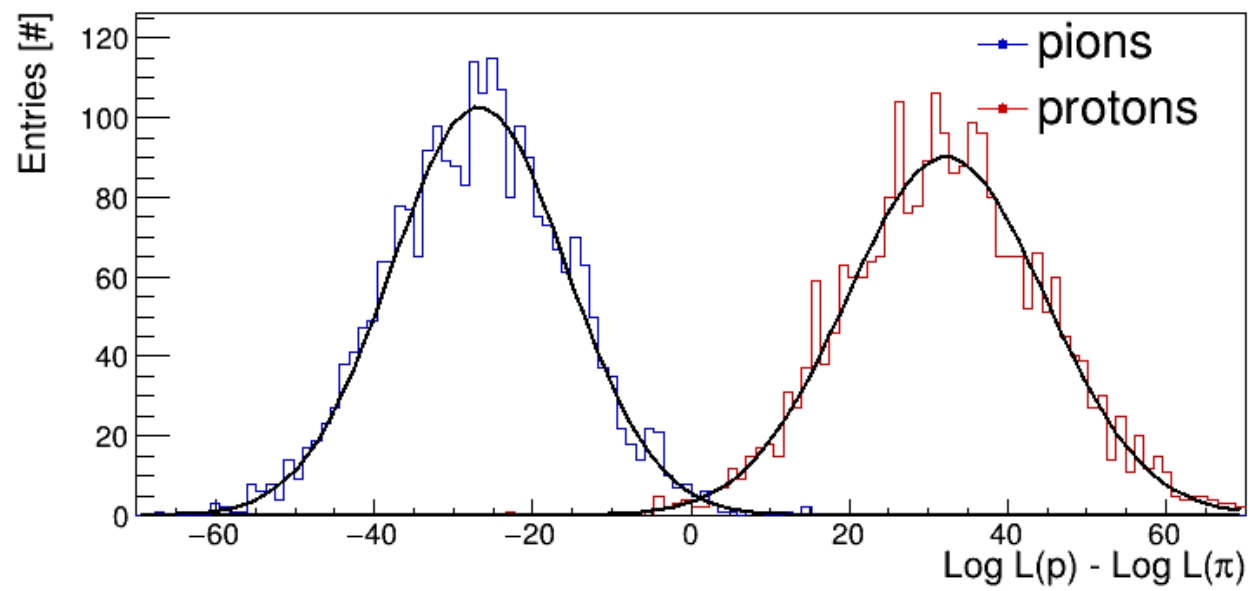

Figure 3.24: Example of the $\pi / \mathrm{p}$ log-likelihood difference distributions for 5000 simulated events of protons (red) and pions (blue) as result of the time imaging at $7 \mathrm{GeV} / \mathrm{c}$ momentum and a $20^{\circ}$ polar angle. The $\pi / \mathrm{p}$ separation power value is $5.0 \pm 0.2$ standard deviation. 


\subsubsection{Summary}

For the success of the PANDA physics program, a robust particle identification for a wide momentum range and the full solid angle is mandatory. For this purpose, the Barrel DIRC covers the polar angle range $22^{\circ} \leq \theta \leq 140^{\circ}$. It is designed to cleanly separate kaons with momenta up to $3.5 \mathrm{GeV} / \mathrm{c}$ from a huge pionic background, with a minimum $\pi / \mathrm{K}$ separation power of 3 standard deviations. The design of the PANDA Barrel DIRC was inspired by the successful BABAR DIRC counter with several key improvements. These improvements are essential to be tested experimentally. For that purpose, the prototype program aims to show if the expected performance in the simulation can be confirmed experimentally. The near finaldesign of the PANDA Barrel DIRC was implemented in the stand-alone prototype simulation. The Geant4 simulation was developed for the beam test, incorporating many features of the PANDA Barrel DIRC Geant4 simulation, using the same physics reactions, materials properties tables and hadronic beam properties at T9.

Several reconstructions and PID algorithms were developed for the PANDA Barrel DIRC and implemented into the prototype simulation framework. The geometrical reconstruction delivers a measurement of the single photon Cherenkov angle resolution, the Cherenkov angle of the track, and the photon yield, which are essential variables for the DIRC detector performance studies. The geometrical construction is a fast algorithm since the LUTs can be created prior to event reconstruction. The time-based imaging reconstruction approach has performance superior to the geometrical reconstruction, but requires large storage capacities for the PDFs for each angle, position, momentum, and particle type, unless the PDFs can be calculated analytically. The analytical PDFs have not been implemented for PANDA yet and it remains to be seen if the performance is competitive with the results from the standard time-based method. The comparison between the simulation and experimental data for the prototype test at CERN is discussed in section 4.8.4. 


\section{Chapter 4}

\section{The PANDA Barrel DIRC Prototype Test Beam}

\subsection{Introduction}

Several beam test campaigns were carried out at CERN and GSI to evaluate the performance of different design options (e.g., photon sensors, focusing lenses, radiators, and expansion volumes) and to validate the PID performance [15]. The simulations were used to generate the look-up tables (LUT) for the geometrical reconstruction, for design optimization, and to compare the results with beam data. Details about the prototype simulation are described in section 3.5. The prototype had undergone through the evolution of different specific tests that were performed at GSI and CERN to validate the design, the optical components, the electronics, and the simulation. The latest prototype development was an attempt to modify the TDR design by reducing the number of photo-detectors leading to considerable overall cost savings. For that purpose, the prototype was placed at the particles beam at CERN. The prototype evolution during the last decade is described in detail at Ref. [15].

\subsection{Test Beam Setup}

During the most recent beam test campaign, in the summer of 2018, the prototype was placed into the mixed hadron beam in the T9 experimental zone [36] at the CERN PS. The data were taken during approximately four weeks, accumulating roughly one billion triggers, including scans of the polar and azimuthal angles at different momentum points with several geometrical configurations.

\subsubsection{Test Beam Facility}

A proton beam coming from the PS accelerator hits a fixed target. A variety of particles are produced as a result of the interactions. Therefore, the collision product is a mixed hadron beam, mainly protons and pions. The T9 [36] beamline can transport the charged particles with momenta between $0.5 \mathrm{GeV}$ and $10 \mathrm{GeV}$. Depending on PS accelerator scheduling, the beam bunches are delivered uniformly to the T9. The maximum particle rate per single bunch is about $10^{6}$ for a $7 \mathrm{GeV} / \mathrm{c}$ 
beam momentum but drops for lower momenta. The charged particles travel approximately $55 \mathrm{~m}$ to reach the experimental area. The experiment setup is located in an area of about $5 \mathrm{~m}$ by $12 \mathrm{~m}$, containing several sub-systems, which are placed along the beamline to tune, analyze and measure the properties of the beam and its composition.

\subsubsection{Prototype Setup}

The Barrel DIRC prototype (see Fig. 4.1) was placed between two time-of-flight (TOF) detectors that were spaced about $28.6 \mathrm{~m}$ apart and used for $\pi / \mathrm{p}$ tagging. A CAD drawing of the experimental setup in the T9 [36] hall can be seen in Fig. 4.2. A scintillating fiber hodoscope delivered charged track position information. Three scintillator counters (Trigger 1, Trigger 2 Vertical, and Trigger 2 Horizontal in Fig. 4.2) were placed in front of and behind the prototype, which has the ability to provide redundant and robust triggering and tracking functions.

A schematic drawing of the setup is illustrated in Fig. 4.3.

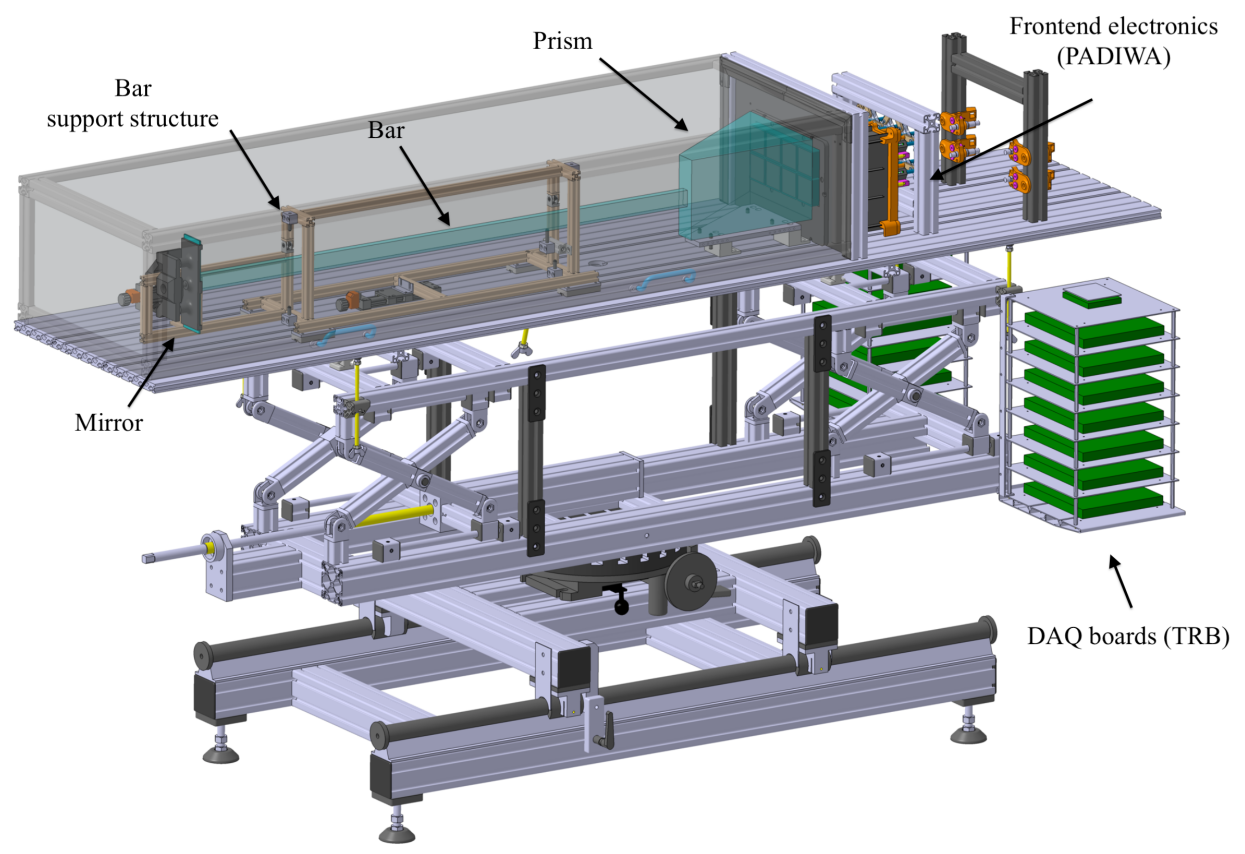

Figure 4.1: Schematic drawing of the prototype.

Cherenkov photons, emitted along the charged particle track in a fused silica bar $\left(17.15 \times 34.93 \times 1200.06 \mathrm{~cm}^{3}\right)$, are transported via total internal reflection inside the bar. A flat front-coated mirror is attached to one end of the bar to reflect photons towards the other end, where a 3-layer spherical lens (made from fused silica and lanthanum crown glass) focuses the photons on the back of a fused silica prism with $300 \mathrm{~mm}$ depth and an opening angle of $33^{\circ}$, serving as expansion volume. A $2 \times 4$ array of PHOTONIS Planacon Microchannel Plate Photomultiplier Tubes (MCP-PMTs) [37] with a total of 512 pixels $\left(6 \times 6 \mathrm{~mm}^{2}\right.$ each $)$ is attached to the prism and held in place by a $3-\mathrm{D}$ printed support structure. The optical coupling between the bar/lens, lens/prism, and prism/MCP-PMTs was made using Eljen EJ-550 optical grease [38]. Figure 4.4 shows examples of the optical coupling for 

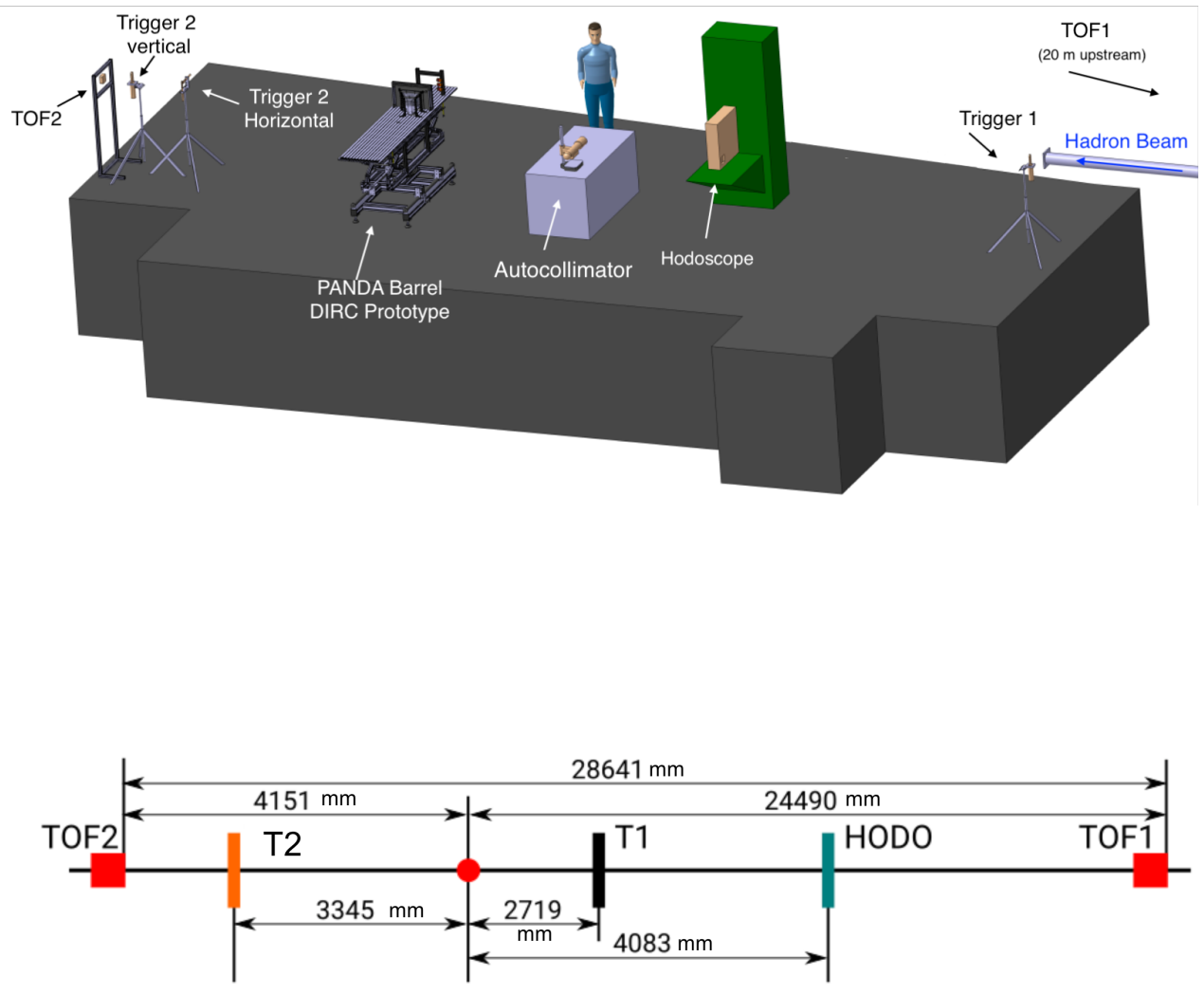

Figure 4.2: CAD drawing of the T9 experimental hall with the PANDA DIRC prototype setup.

some parts. The MCP-PMTs were read out by an updated version of the Trigger and Readout Board (TRB) [39], initially developed for the HADES experiment [40], in combination with the PADIWA front-end amplification and discrimination card [41]. The mechanical system of the prototype was designed with a rotating table and rails that allow the detector to be rotated and translated relative to the beamline. The prototype rotation was remote-controlled and monitored by a camera. The radiator was carefully held in place by a pair of 3-D printed plastic braces equipped with screws, which allowed fine adjustments of the bar position. The optical components of the prototype were covered by a black box to make the setup light-tight. The alignment of all components of the prototype at T9 was performed by an autocollimator and a laser alignment system.

\subsection{Event Selection and Particle Tagging}

Since the direct evaluation of $\pi / \mathrm{p}$ separation at $3.5 \mathrm{GeV} / \mathrm{c}$ was not feasible, the PID performance was evaluated for $\pi /$ p at $7 \mathrm{GeV} / \mathrm{c}$ instead. At $7 \mathrm{GeV} / \mathrm{c}$ momentum, the 


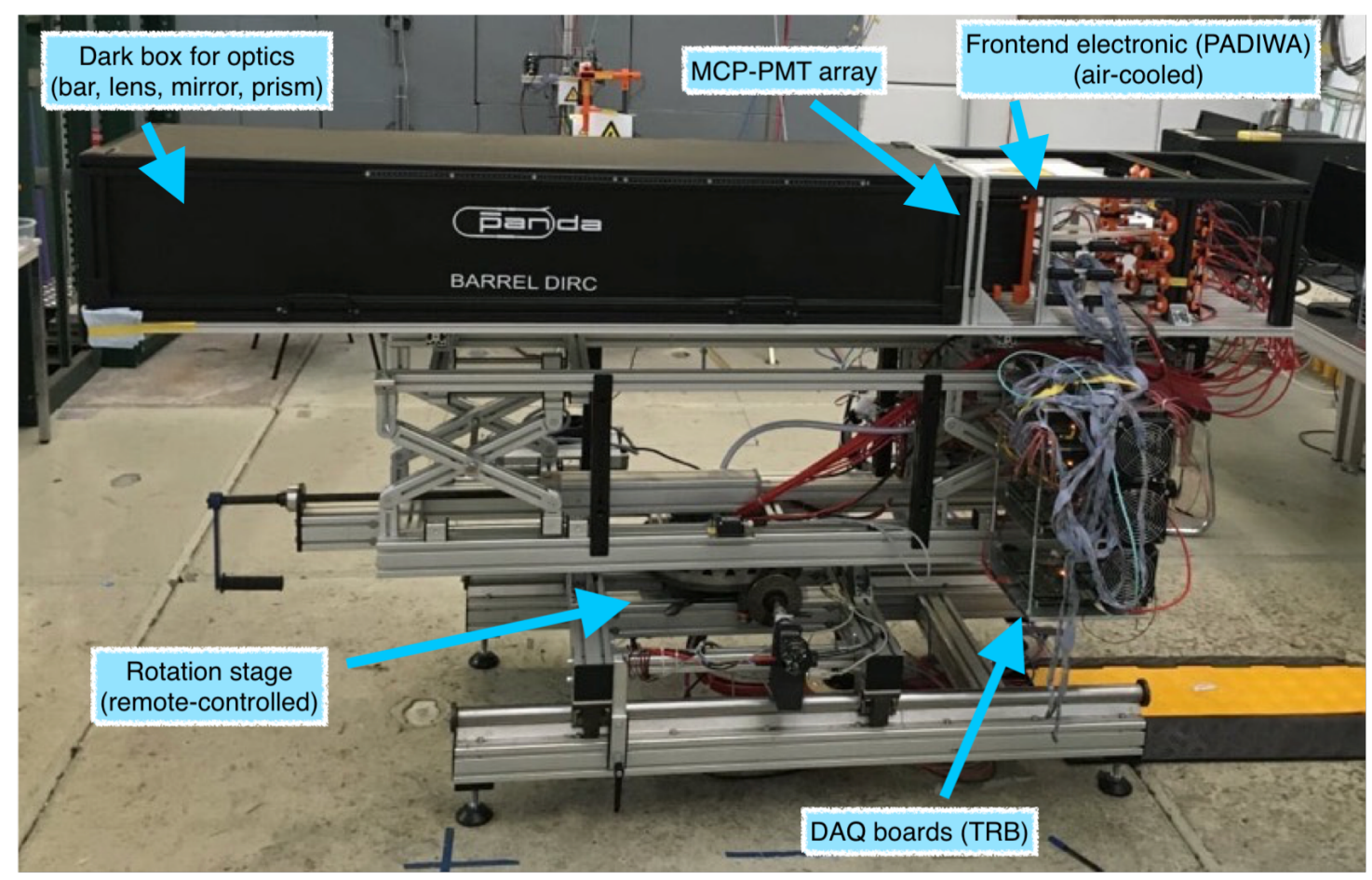

Figure 4.3: Photo of the PANDA Barrel DIRC prototype in the T9 beamline at CERN

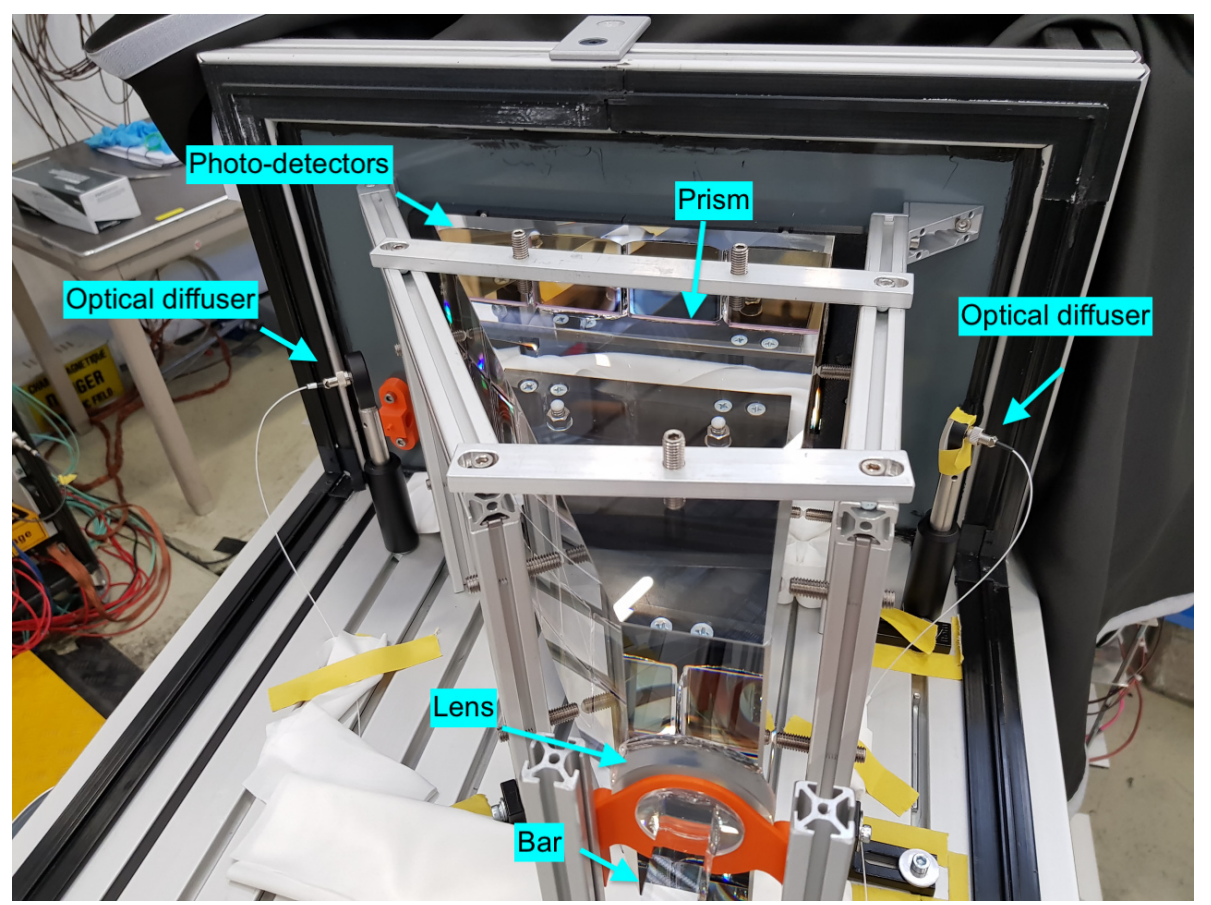

Figure 4.4: Photos of the optical system.

Cherenkov angle difference between pions and protons $(8.1 \mathrm{mrad})$ is approximately equivalent to the Cherenkov angle difference between pion and kaon at $3.5 \mathrm{GeV} / \mathrm{c}$ $(8.5 \mathrm{mrad})$, the upper limit of the PID performance range of the PANDA Barrel DIRC. 
The beam cores-section has an elliptical shape. The horizontal and the vertical beam profiles covered ranges of about $\pm 50 \mathrm{~mm}$ with RMS values of about $26 \mathrm{~mm}$ and 16 $\mathrm{mm}$, respectively. The Trigger 1 signals were used by the Data Acquisition (DAQ) system for event recording. The coincidence between the trigger signals (Trigger 1, Trigger 2 Vertical, and Trigger 2 Horizontal) was used for event selection. Also, the selected events require at least one hit for each of the vertical and horizontal fibers of the scintillating hodoscope. Two time-of-flight (TOF) detectors were separated by $29 \mathrm{~m}$ and used for particle tagging to provide external $\pi / \mathrm{p}$ identification. Figure 4.5 shows beam particles time of flight measured by the two TOF stations. Clear peaks for the pions and protons were observed for beam data at momentum of $7 \mathrm{GeV} / \mathrm{c}$. The event selection criteria of the events were based on the TOF system, which aims to provide as clean as possible testing samples to evaluate the PID of the DIRC. This is done by removing the tails to exclude the events that, for unknown reasons, have too long a propagation time of flight, and also by removing the contaminated region in between the two peaks to obtain as pure as possible testing samples. The selection cut was defined by the vertical lines in Fig. 4.5.

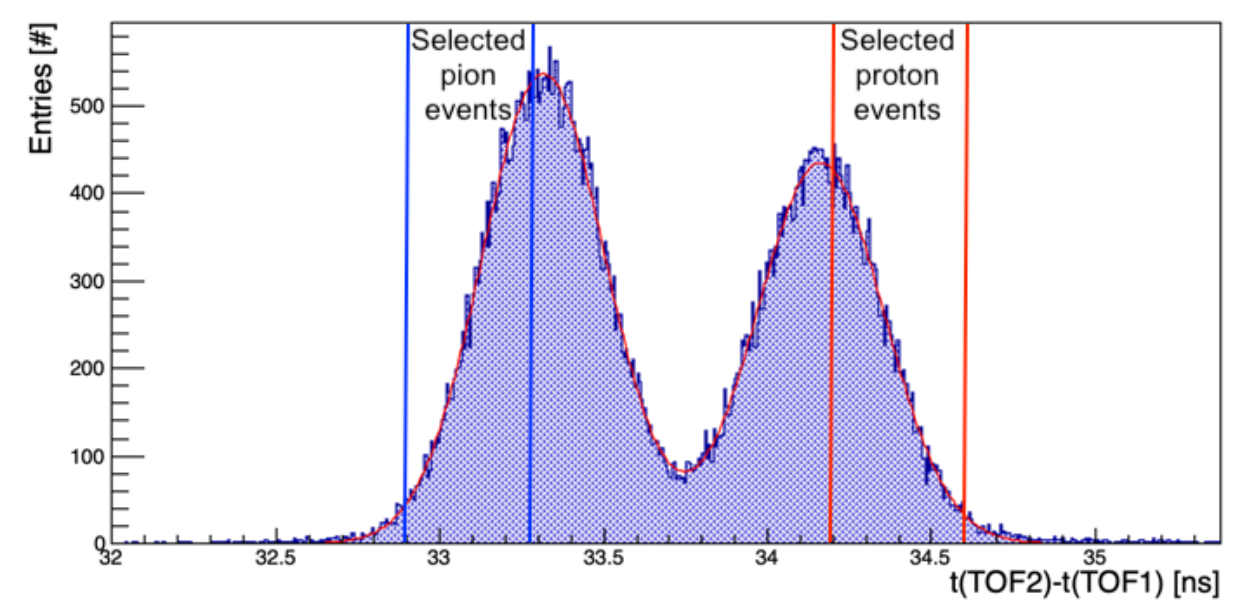

Figure 4.5: Beam particles time of flight measured by the two TOF stations.

The Geant 4 simulation was fine-tuned to match the properties of the selected events. The simulated particle beam was adjusted to have a $1 \mathrm{~cm}$ beam diameter to match the beam profile after applying the auxiliary detector selection with $1.5 \mathrm{mrad}$ divergence. In the following sections, the test beam results and Geant4 simulation are discussed using the geometrical reconstruction and the time imaging PID algorithms.

\subsection{Hit Pattern}

Figures 4.6a and Fig. 4.7a show the accumulated hit pattern for pions and protons using beam data recorded with a polar angle of $20^{\circ}$ and $7 \mathrm{GeV} / \mathrm{c}$ and with a threelayer spherical lens. The single event hit pattern for both proton and pions are shown in Fig. 4.6b and Fig. 4.7b respectively. Since the angular separation of pions and protons at $7 \mathrm{GeV} / \mathrm{c}$ momentum is about $8 \mathrm{mrad}(\approx 1 / 3$ of a pixel $)$, which make it impossible to distinguish between pions and protons from one single photon hit pattern, instead the reconstruction methods combine the information (timing and position) form all of the Cherenkov photons to perform the PID. 
(a) Accumulated proton event hit pattern

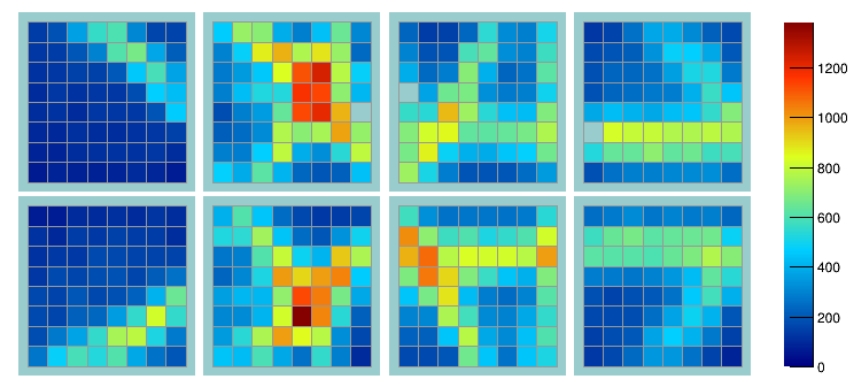

(b) Single proton event hit pattern

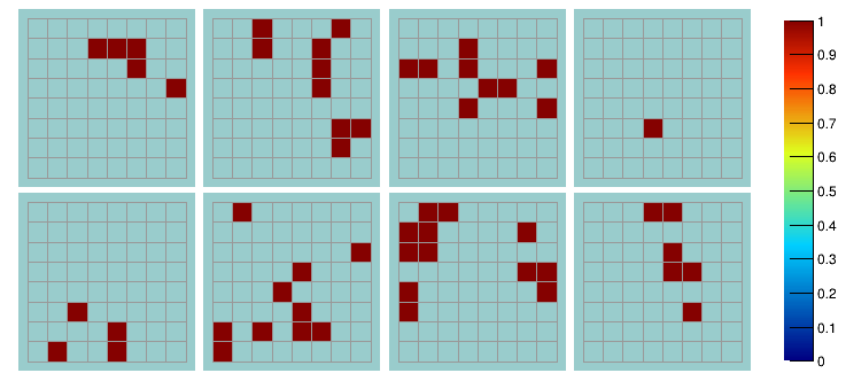

Figure 4.6: a) Accumulated hit pattern for proton beam data at $7 \mathrm{GeV} / \mathrm{c}$ momentum with $20^{\circ}$ polar angle using 4000 events. b) Single event proton hit pattern.

(a) Accumulated pion event hit pattern

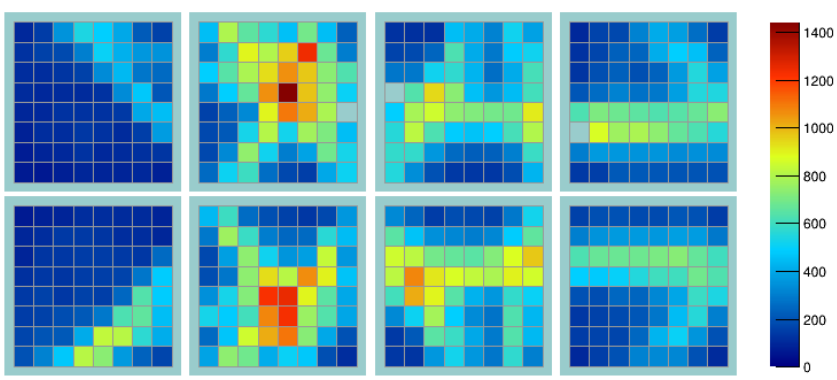

(b) Single pion event hit pattern

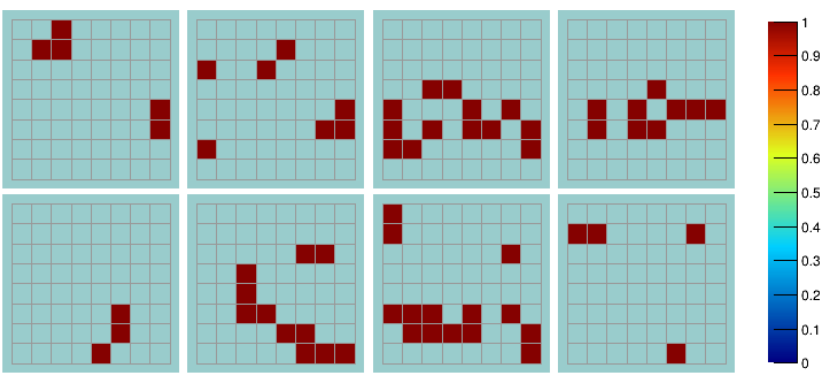

Figure 4.7: a) Accumulated hit pattern for pion beam data at $7 \mathrm{GeV} / \mathrm{c}$ momentum with $20^{\circ}$ polar angle using 4000 events. b) Single event pion hit pattern. 


\subsection{Timing Calibrations}

The calibration of each readout channel was performed using a $405 \mathrm{~nm}$ Picosecond laser pulser (PiLas) [42]. The trigger output from the laser pulser was used as a reference time. Single-mode optical fibers transported the light to two optical diffusers to evenly illuminate the entire MCP-PMT plane, as shown in Fig. 4.4. Pixel-to-pixel time offsets due to differences in cable lengths and internal delays on the readout cards were adjusted using data recorded with the Picosecond Laser pulser (PiLas).

The data from the prototype are stored in the data format of the HADES data acquisition system protocol (TrbNet) [39], and converted offline into the CERN ROOT data format [43] for analysis. The most important information stored in the analysis file are the times of the leading and trailing edge of the detected signals [15] (see Fig. 4.8).

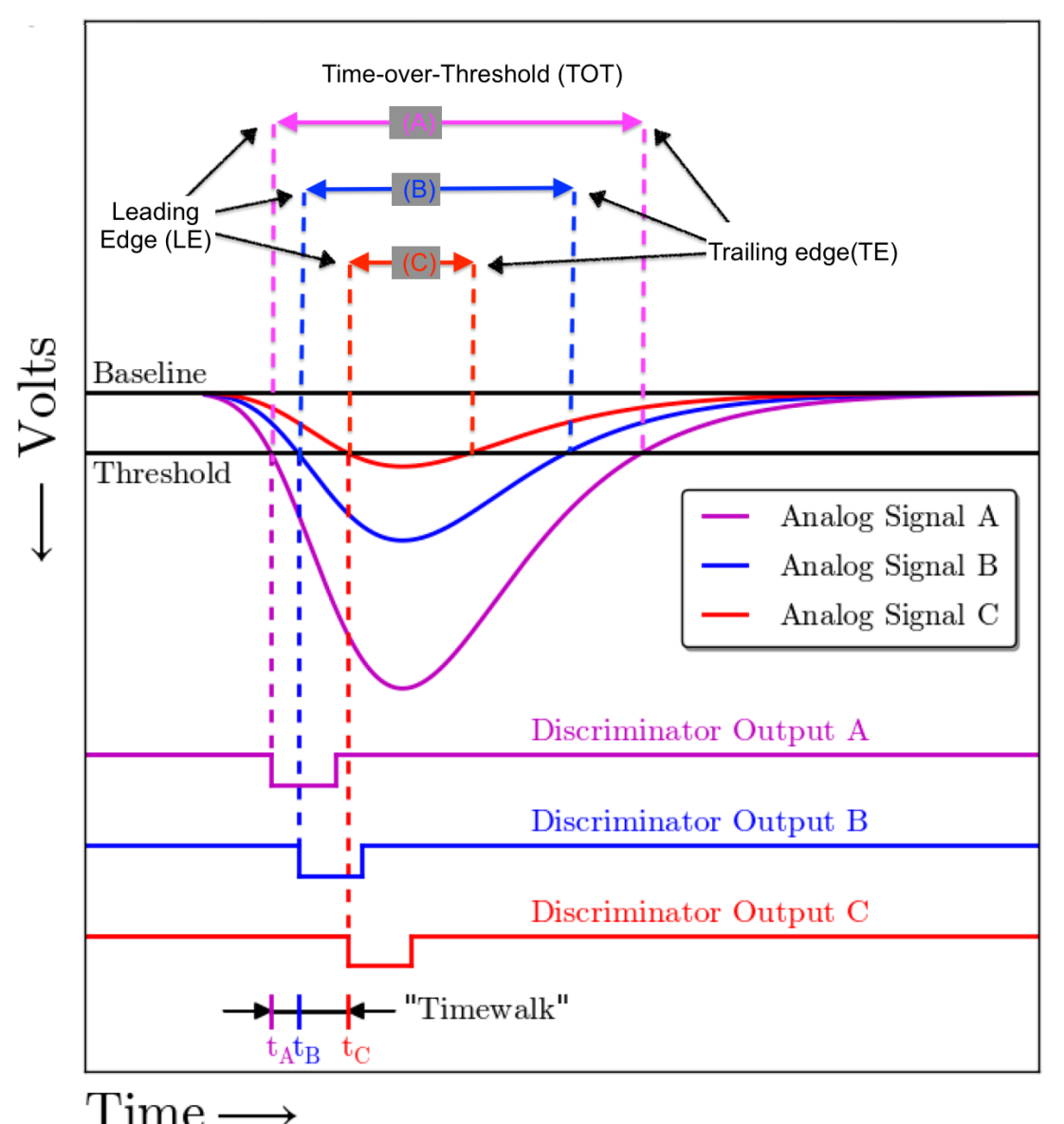

Figure 4.8: Schematic drawing of the analog signals from a photo-detector channel [44].

Each time is stored in three variables: The EPOCH counter (with a range of 45.8 min), the COARSE counter $(10.24 \mu \mathrm{s})$, and the FINE counter with a range of approximately 5 ns [15]. For each pixel, the photon arrival time spectrum in laser data was fitted, and the mean values stored in a database as shown in Fig. 4.9. These 
time offsets were calculated for each prototype configuration and subtracted from the photon time to align all pixels in time-space.

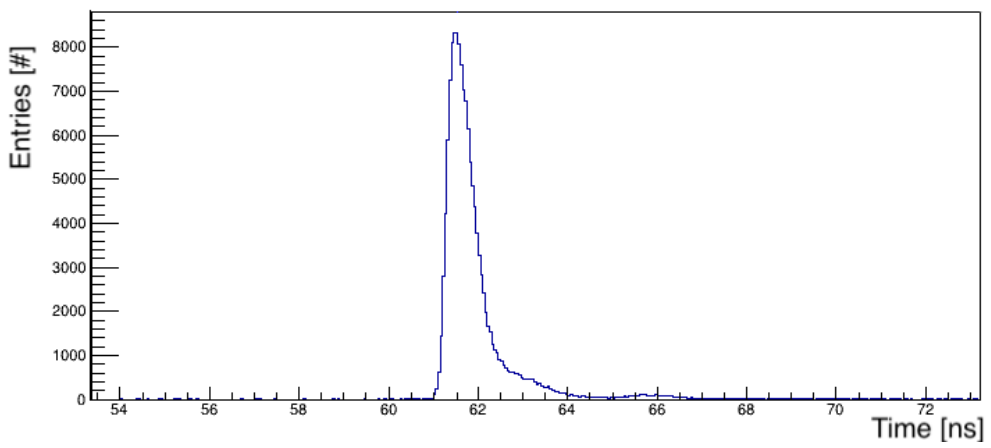

Figure 4.9: The photon leading edge time for one pixel using laser data.

Since the analog pulses have different rise times, this forbids simple threshold triggering, which causes a dependence of the trigger time on the peak amplitude of the signal, an effect called time walk as shown in Fig. 4.8. The Time-over-Threshold (TOT) is related to the pulse height of the analog signal and can be used to observe the photo-sensor performance and to perform time-walk corrections to reach the required photon timing precision. Fig. 4.10 shows a clear peak around $3 \mathrm{~ns}$ for the corrected Time-over-Threshold (TOT) after the removal of the time offset (i.e., trailing edge - leading edge - time delay ) for one pixel. It was expected to find one single peak. However, there are secondary peaks observed on the right and left of the main peak. They are potentially caused by the electronics oscillation [45].

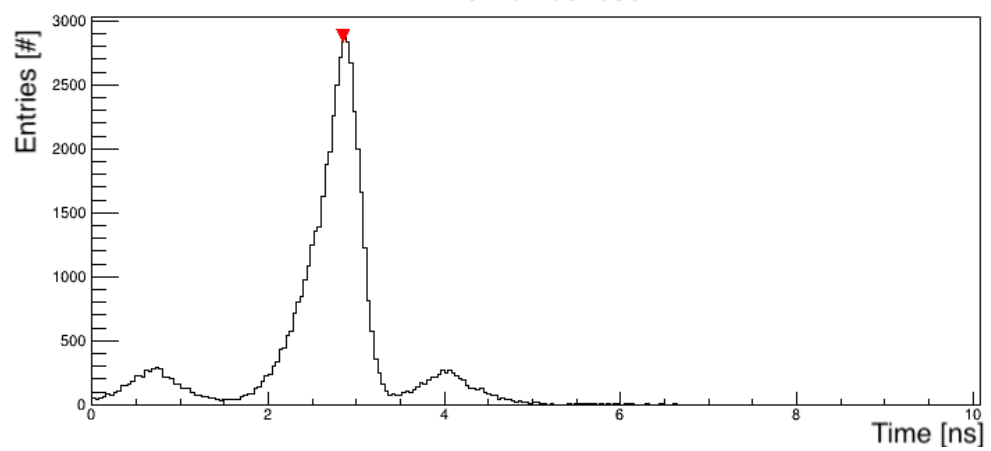

Figure 4.10: Corrected time-over-threshold (TOT) for one pixel using laser data.

Figure 4.11 shows the leading edge time as a function of TOT for data taken with the PiLas. A clear peak can be observed and the secondary peaks are due to the electronics oscillation described above. The time walk shows a correlation with the TOT. This correlation is corrected by creating a histogram for each bin on the y-axis. These histograms were fitted by Gaussian function. The correction is performed by subtracting the mean of the fit for each histogram from the mean of the entire distribution.

Figure 4.12 shows the leading edge time resolution for one pixel. The two histograms and the corresponding fits to the data show the results before and after a time walk correction using the TOT information, demonstrating the significant time precision 

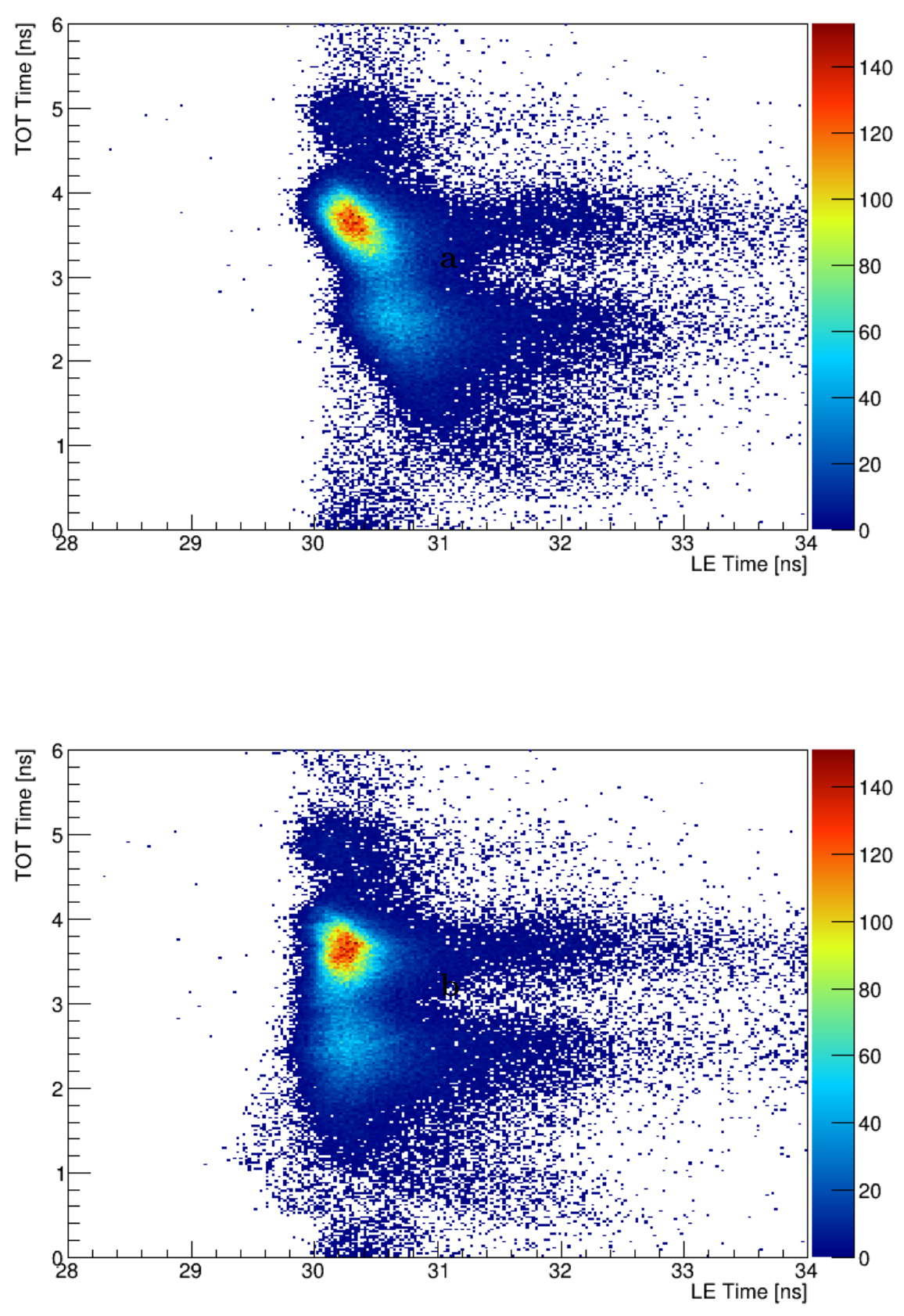

Figure 4.11: Time-over-Threshold (TOT) as function of Leading Edge (LE) time for one single pixel: before applying the time-walk correction (a), and after applying the time-walk correction (b). 
improvement. The time walk correction was successfully applied to 512 pixels and an average time precision below 190 ps was obtained with an improvement of about $15 \%$.
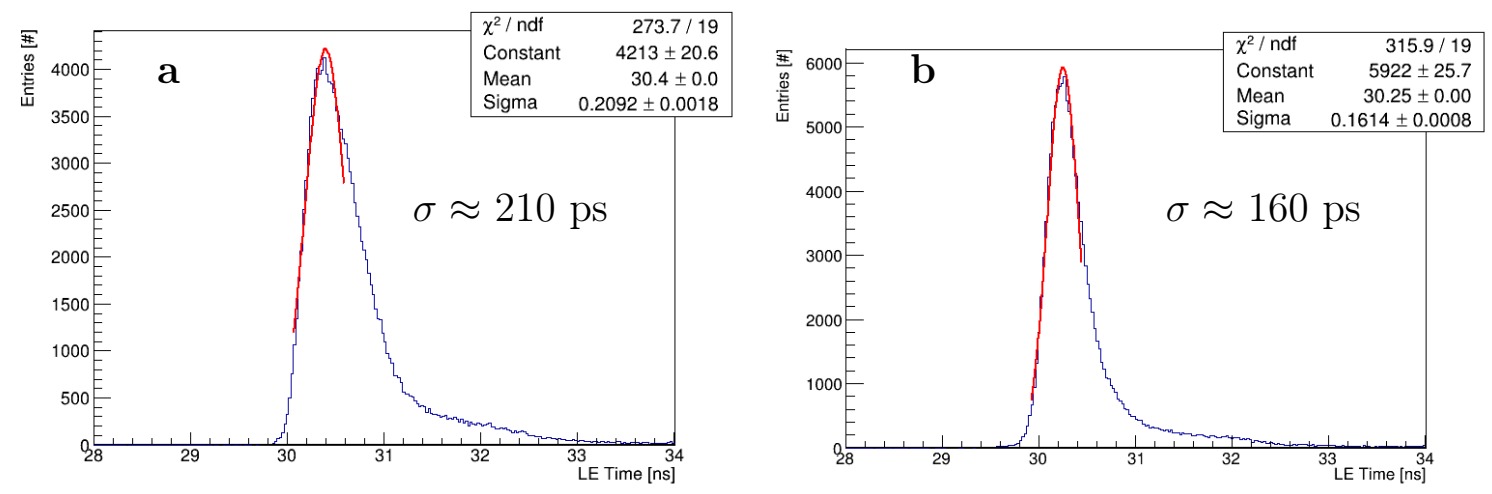

Figure 4.12: Example of the photon arrival time for one pixel: before applying the time-walk correction (a), and after applying the time-walk correction (b). The peak is fitted with a Gaussian function, the tail is caused by electrons back-scattered within the MCP-PMT.

Figure 4.13 shows the time resolution distribution for 512 pixels before time-walk correction (blue) and after time-walk correction. Finally, the event time offset was subtracted using simulation to facilitate the comparison of the experimental data to simulation.

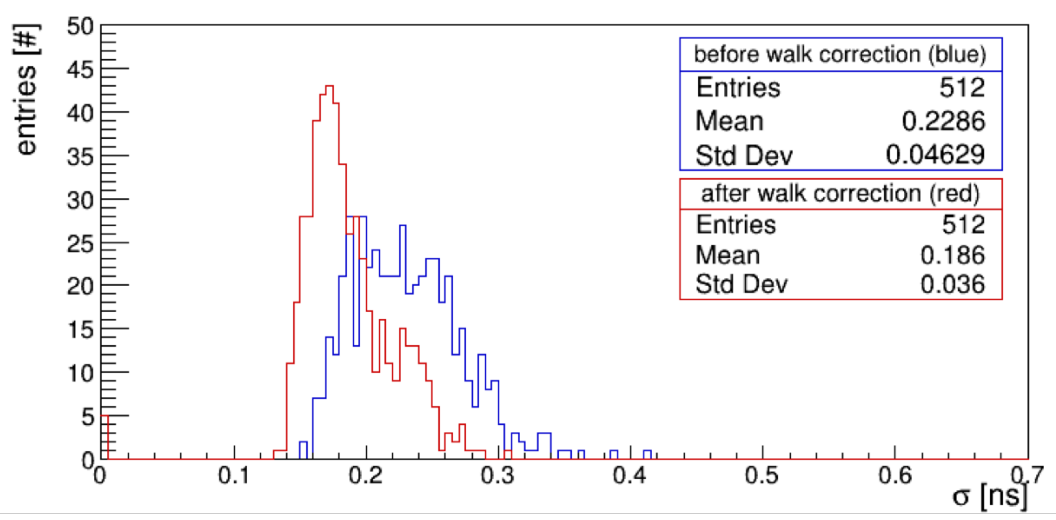

Figure 4.13: Time resolution distribution for 512 pixels before time-walk correction (blue) and after time-walk correction (red). 


\subsection{PID Performance}

Figure 4.14 shows the Cherenkov angle distribution per photon for one single proton track. The distribution was obtained after applying the time cut to reduce the reconstruction ambiguities. The time cut is described in section (4.7.1). A clear peak can be found at the expected value of the Cherenkov angle in the top of the reconstruction ambiguities background. The Cherenkov angle per photon distribution is fitted by a Gaussian plus a linear background.

\section{proton-tagged beam data}

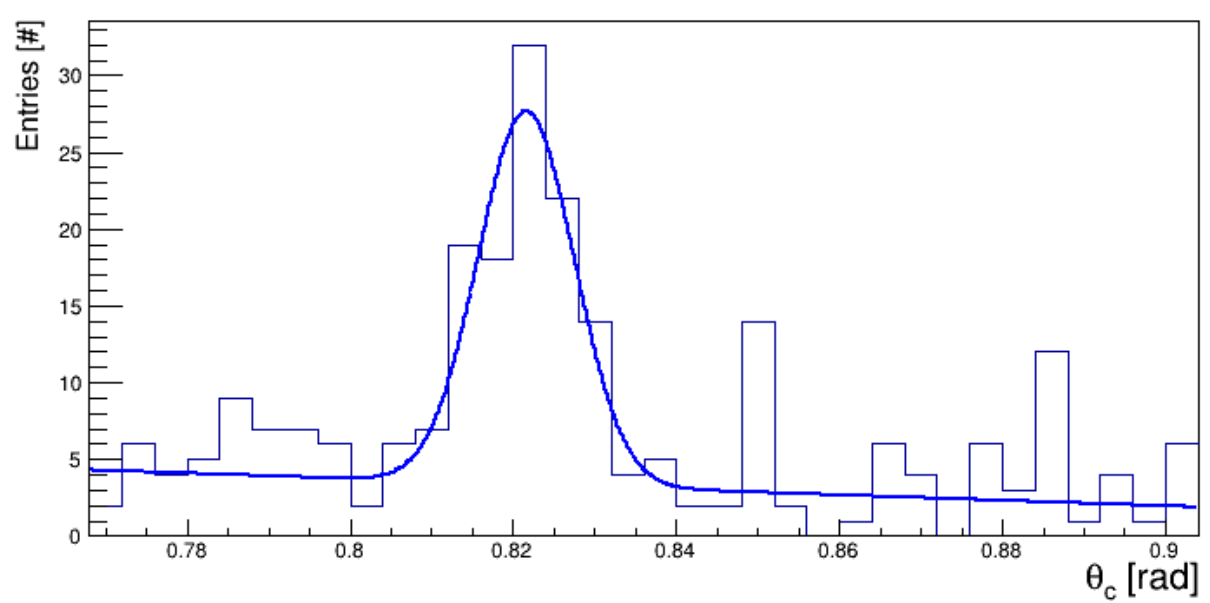

Figure 4.14: Examples of the reconstructed Cherenkov angle per photon for one single proton-tagged track beam data at $7 \mathrm{GeV} / \mathrm{c}$ momentum and $20^{\circ}$ polar angle.

The Cherenkov angle resolution per track $\sigma_{C, \text { track }}$ can be determined from the width of the difference between the measured and the expected Cherenkov angles distribution. Fig. 4.15 shows the result of the track-by-track Cherenkov angle fit for proton-tagged beam data, for a $7 \mathrm{GeV} / \mathrm{c}$ momentum at $20^{\circ}$ angle. The observed Cherenkov angle resolution per particle $\sigma_{\text {C.track }}$ has a value of $2 \mathrm{mrad}$. By combining this value with the Cherenkov angle difference for pion and proton at $7 \mathrm{GeV} / \mathrm{c}$ momentum, this is translated into 4 s.d. separation, which corresponds to about 4.3 s.d. $\pi / \mathrm{K}$ separation. In the following sections, the PID and performance optimization of the prototype at the CERN PS 2018 test beam is introduced using geometrical reconstruction and time-based imaging.

\subsection{Geometrical Reconstruction}

The geometrical reconstruction method combines the location and direction of the charged particle track in the bar (from the tracking system) and the spatial position of the photon hit at the detection plane (from the MCP-PMTs) to reconstruct the Cherenkov angle and to perform the PID. For a detailed discussion of the geometrical reconstruction, see section 3.4.1. There are three versions of the geometrical reconstruction method used to evaluate the PANDA Barrel DIRC prototype PID performance and will be discussed in the following sections. 
Beam data

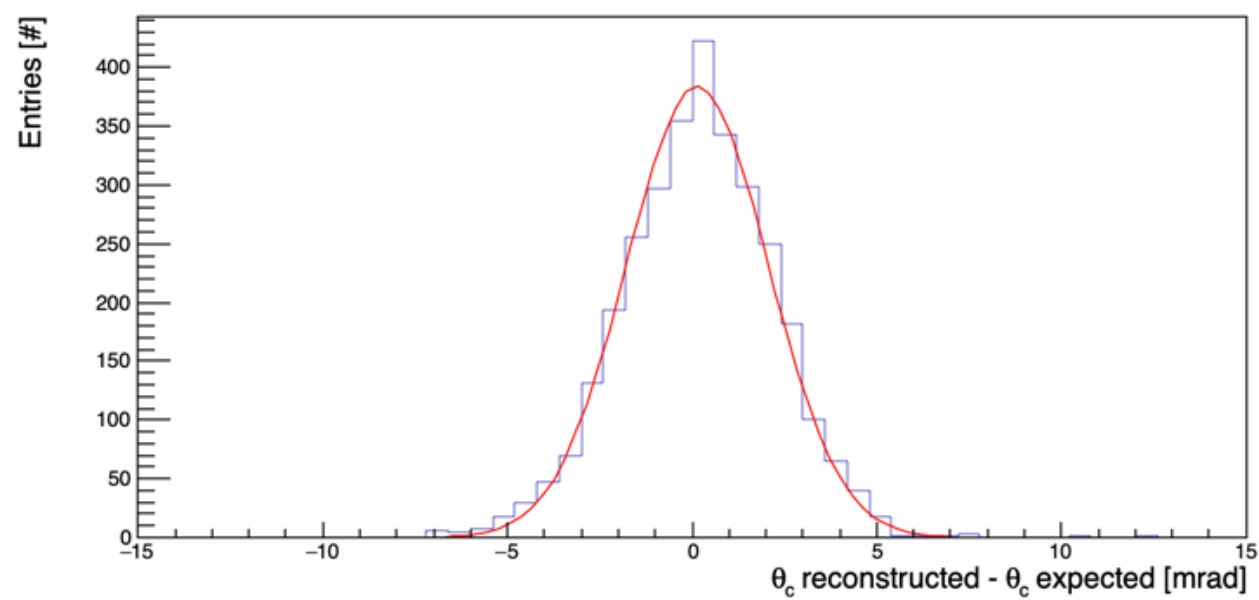

Figure 4.15: Resolution of the reconstructed Cherenkov angle per track for $7 \mathrm{GeV} / \mathrm{c}$ momentum at polar angle of $20^{\circ}$ for 3500 proton-tagged beam data.

- Perform a direct hypotheses test for the single photon Cherenkov angle distribution $\theta_{C, \text { photon }}$ for all hits and ambiguities per track to deliver the loglikelihood difference distribution

- Fit the single photon Cherenkov angle distribution $\theta_{C, \text { photon }}$ per track to determine the mean value $\theta_{C, \text { track }}$, which can be used to calculate the log-likelihood difference distribution

- Create a Cherenkov angle data PDF for each particle type, to deliver the log-likelihood difference distribution

\subsubsection{Event Track Hit Selection}

An example of the time difference distribution of the Cherenkov photons between measured (by the photo-sensors) and calculated time (sum of photon propagation time inside the bar and prism) for proton beam data at $7 \mathrm{GeV} / \mathrm{c}$ momentum at $20^{\circ}$ polar angle is shown in Fig. 4.16a. The distribution shows a very complicated structure that is not Gaussian-like peak, due to the reconstruction ambiguities. The distributions in Fig. $4.16 \mathrm{~b}$ were obtained after applying $\pm 50 \mathrm{mrad}$ cut around the expected value of the Cherenkov angle, to suppress the reconstruction ambiguities. The peaks at the sides due to the remaining ambiguities of the photon path, that cause significant time difference around the main peak.

Figure 4.17a illustrates a two-dimensions selection cut which is used to reduce the reconstruction combinatorial background and to compensate the chromatic dispersion. The selected solutions are used to reconstruct the Cherenkov angle and to perform the PID. The time cut impact on the selected photon path solution and the proton hit pattern is shown in Fig. 4.16c and Fig. 4.17b respectively. 

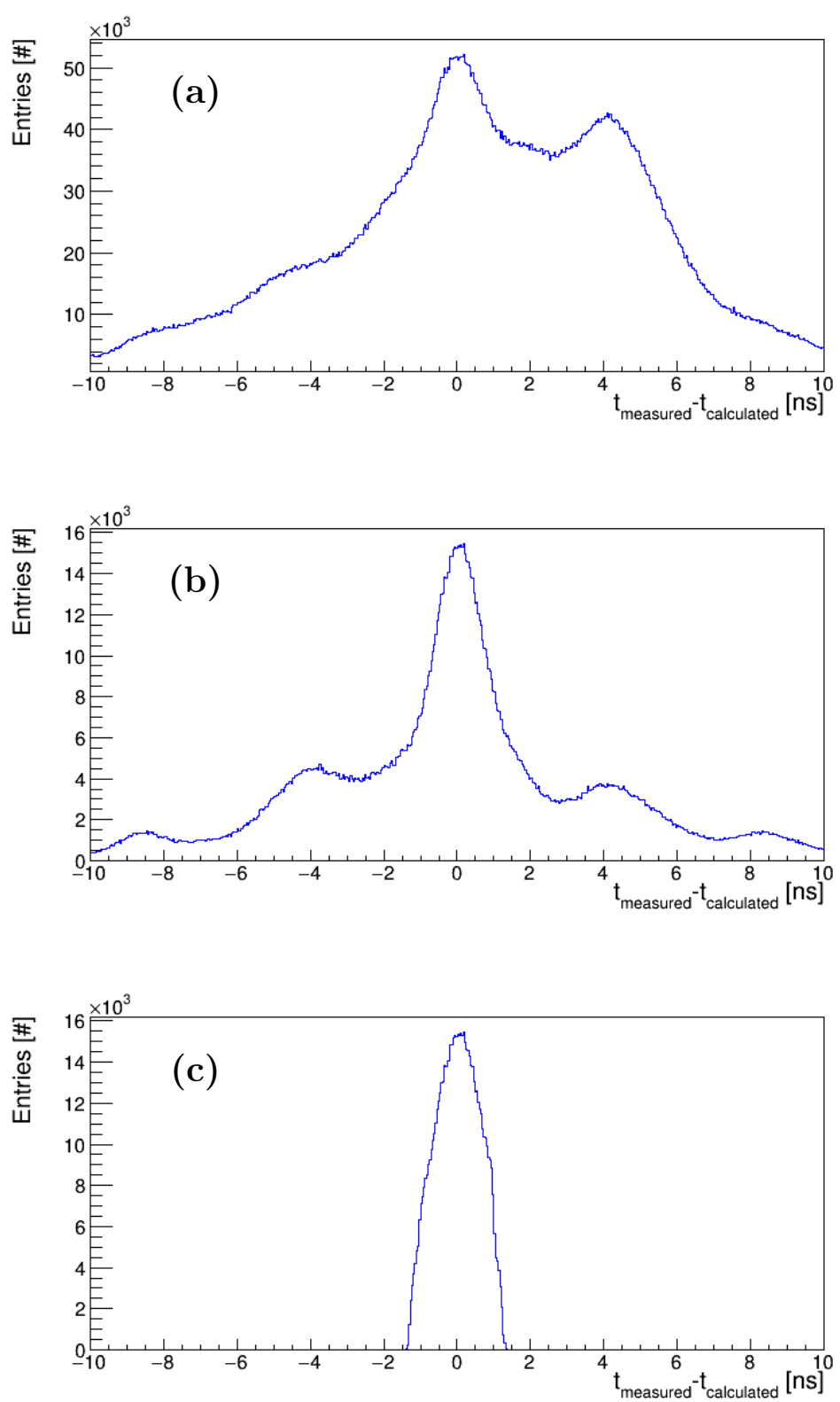

Figure 4.16: Time difference distribution between measured and expected arrival time of the Cherenkov photons path solutions (a), after applying the Cherenkov angle cut (b), and after applying the Cherenkov angle cut and the 2D time difference cut discriped in Fig. 4.17a.

\subsubsection{Determination of the Cherenkov Angle Resolution per Photon}

Figure 4.18 shows an example of the reconstructed Cherenkov angle per photon from proton-tagged and pion-tagged beam data at $7 \mathrm{GeV} / \mathrm{c}$ momentum at $20^{\circ}$ polar angle using approximately 8000 events. Both distributions were obtained after applying the time cut described above to suppress the combinatorial background. A clear peak can be seen at the expected value of the Cherenkov angle on top of the reconstruction ambiguities background. The Cherenkov angle per photon distribution is fitted by a Gaussian plus a second-order polynomial background. The fitting 

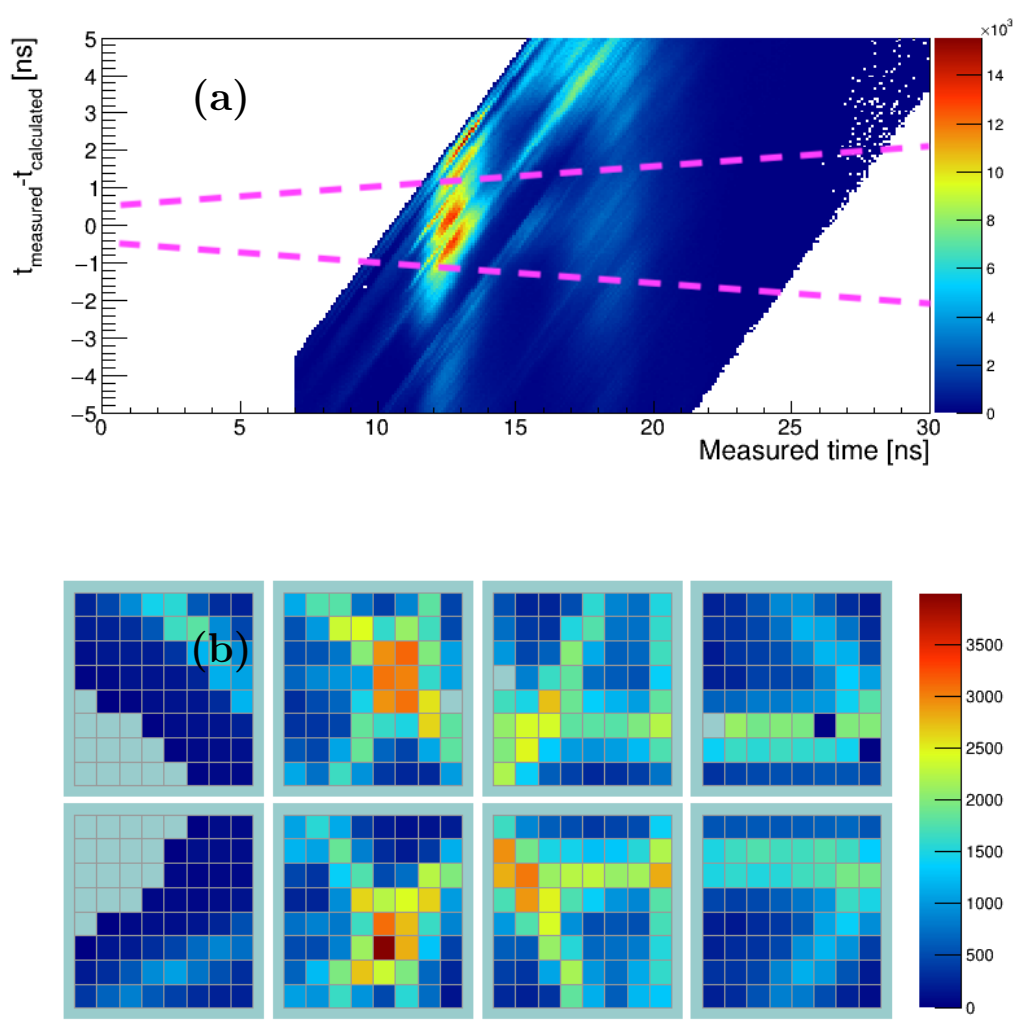

Figure 4.17: Two-dimensional time difference cut defined by the magenta lines (a). Effect of the time difference cut on the hit pattern (b).

result indicates that the Cherenkov angle resolution per photon for the proton and pion is about $7.9 \mathrm{mrad}$ and 7.4 , respectively.

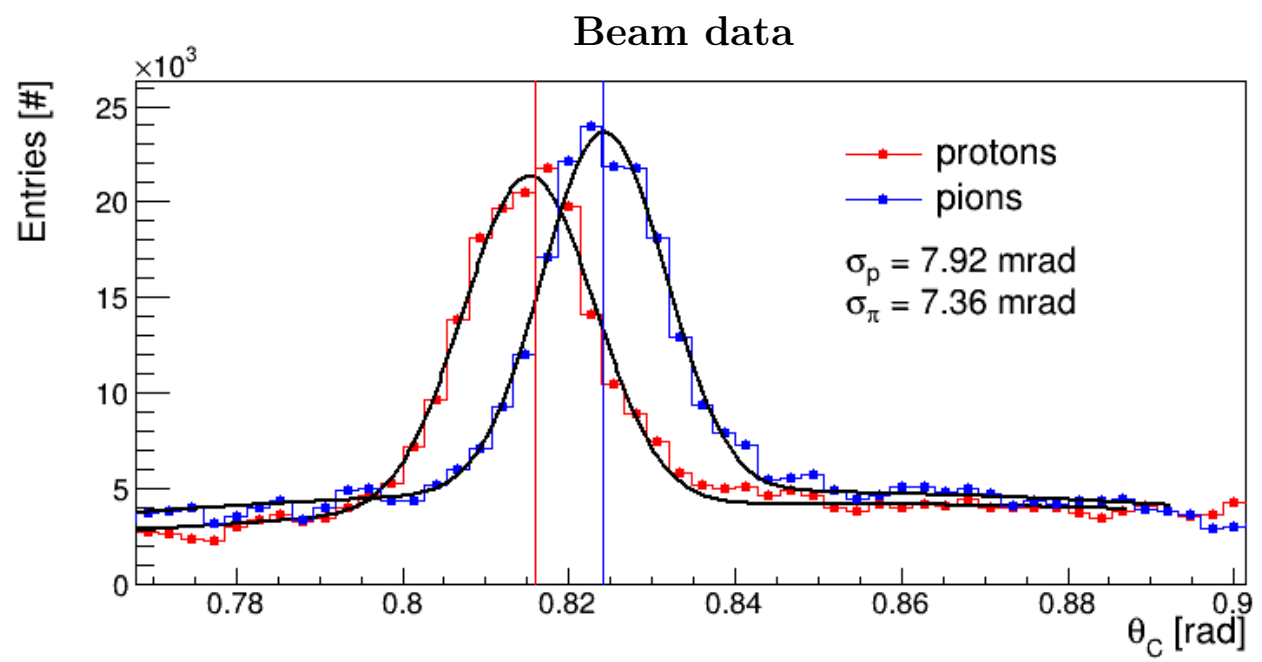

Figure 4.18: Examples of the reconstructed Cherenkov angle per photon from proton-tagged (red) and pion-tagged (blue) beam data at $7 \mathrm{GeV} / \mathrm{c}$ momentum and $20^{\circ}$ polar angle. 


\subsubsection{Photon Yield}

Photon yield is an important quantity that is used to study the DIRC detector performance. The determination of the number of detected photons per particle is obtained by counting the survival photons for each track after applying the 2D time cut described above and a Cherenkov angle cut around the expected value within $\pm 50 \mathrm{mrad}$. Figure 4.19 shows photon yield per track for proton-tagged and pion-tagged beam events as a result of the geometrical reconstruction at $7 \mathrm{GeV} / \mathrm{c}$ momentum and a $20^{\circ}$ polar angle. The average photon yield per track for both pion and proton is the same with excellent agreement simulation.

\section{Photon yield beam data}

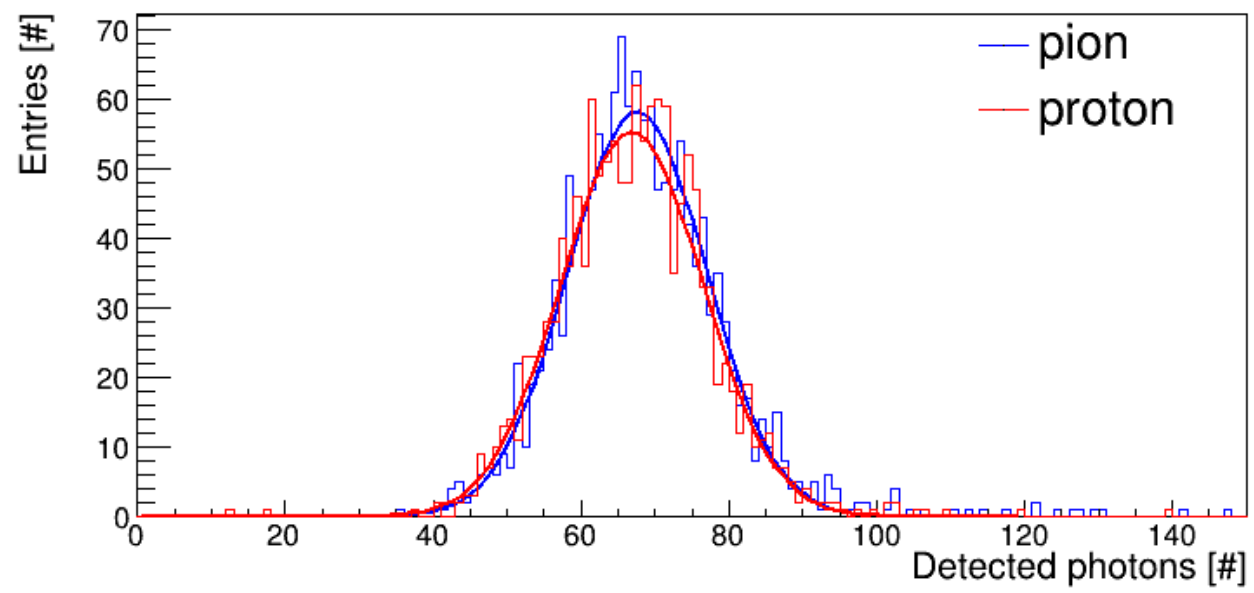

Figure 4.19: Photon yield per track for proton-tagged (red) and pion-tagged (blue) beam events as result of the geometrical reconstruction at $7 \mathrm{GeV} / \mathrm{c}$ momentum and a $20^{\circ}$ polar angle.

\subsubsection{PID Performance}

The distribution of the log-likelihood differences of the pion/proton hypothesis tests for the beam data with $7 \mathrm{GeV} / \mathrm{c}$ and $20^{\circ}$ polar angle is shown in Fig. 4.20. The distribution is fitted with two Gaussians and the $\pi / \mathrm{p}$ separation power is $2.95 \pm 0.2$ standard deviations. This value was obtained for the nominal alignment of the bar and the MCP-PMT based on the measurement which was taken during the data taking. However, the separation power can be further improved using several correction procedures, which will be explained in the following sub-sections.

\subsubsection{PID Performance Optimization}

\section{Charged Track Direction Correction}

The alignment process was performed using an autocollimator and a laser alignment system relative to the nominal beamline. Given that there is no way to know the true beam direction, this can cause beam direction misalignment. The prototype was aligned relative to the nominal beamline at a 90-degree polar angle. Since the prototype is a rotating object in a 3-dimensional space, once the detector rotated to a different angle, this can cause additional beam direction misalignment. The event selection process can also cause beam direction bias as the event selection was 


\section{Geometrical reconstruction without PID optimization}

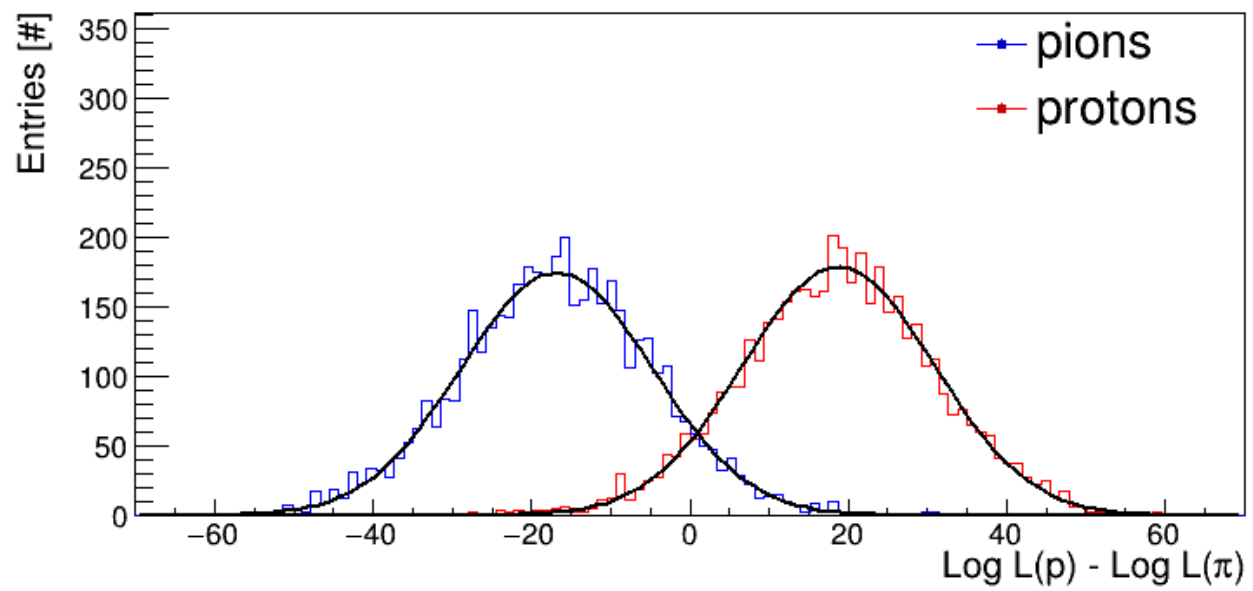

Figure 4.20: $\pi / \mathrm{p}$ log-likelihood difference distributions for proton-tagged (red) and pion-tagged (blue) beam events $(\approx 8000)$ as result of the geometrical reconstruction at $7 \mathrm{GeV} / \mathrm{c}$ momentum and $20^{\circ}$ polar angle. The $\pi / \mathrm{p}$ separation power value is $2.95 \pm 0.2$ standard deviations.

based on the auxiliary detectors ( TOF 1, TOF 2, Trigger 1, Trigger 2 Horizontal, Trigger 2 vertical, and the hodoscope ). The selected events required that all of the auxiliary detectors be triggered. Their alignments were also done relative to the nominal beamline. This can cause a potential beam direction misalignment.

The beam direction was corrected offline using the data itself. The correction process was carried out by reconstructing the Cherenkov angle to extract the single photon resolution, followed by small adjustments of the beam direction for both polar and azimuthal angles. The corrected direction corresponds to the direction that achieves the best single photon resolution value for every nominal angle. Figure 4.21 shows the single photon resolution (SPR) map as a function of the polar angle and the azimuthal angle rotations. The color scale corresponds to the SPR of proton-tagged data. The white arrow corresponds to the correction vector. The log-likelihood differences hypothesis tests for pion/proton, after applying the charged track direction correction, is shown in Fig. 4.22 for the beam data with $7 \mathrm{GeV} / \mathrm{c}$ and $20^{\circ}$ polar angle. The pion/proton separation is $3.62 \pm 0.2$ standard deviations.

\section{Cherenkov Angle Correction}

Fig. 4.23 shows an example of the reconstructed Cherenkov angle shift for a single MCP-PMT from the expected value. The Cherenkov angle shift per MCP-PMT is mainly due to the mismatch between the implemented positions of the MCP-PMTs in the simulation to create the look-up tables (LUT) and the actual positions during the data taking. The reconstruction method itself can also add a systematic shift to the reconstructed Cherenkov angle. The Cherenkov angle shift for every polar angle and MCP-PMT combinations were calculated based on the proton data. These corrections were applied for both simulation and beam data. Given that all the corrections were kept the same for both protons and pions. Fig. 4.24 shows the $\pi / p$ log-likelihood difference distributions after applying the Cherenkov angle correction per MCP-PMT, the $\pi / p$ separation is $3.83 \pm 0.2$ standard deviations. 


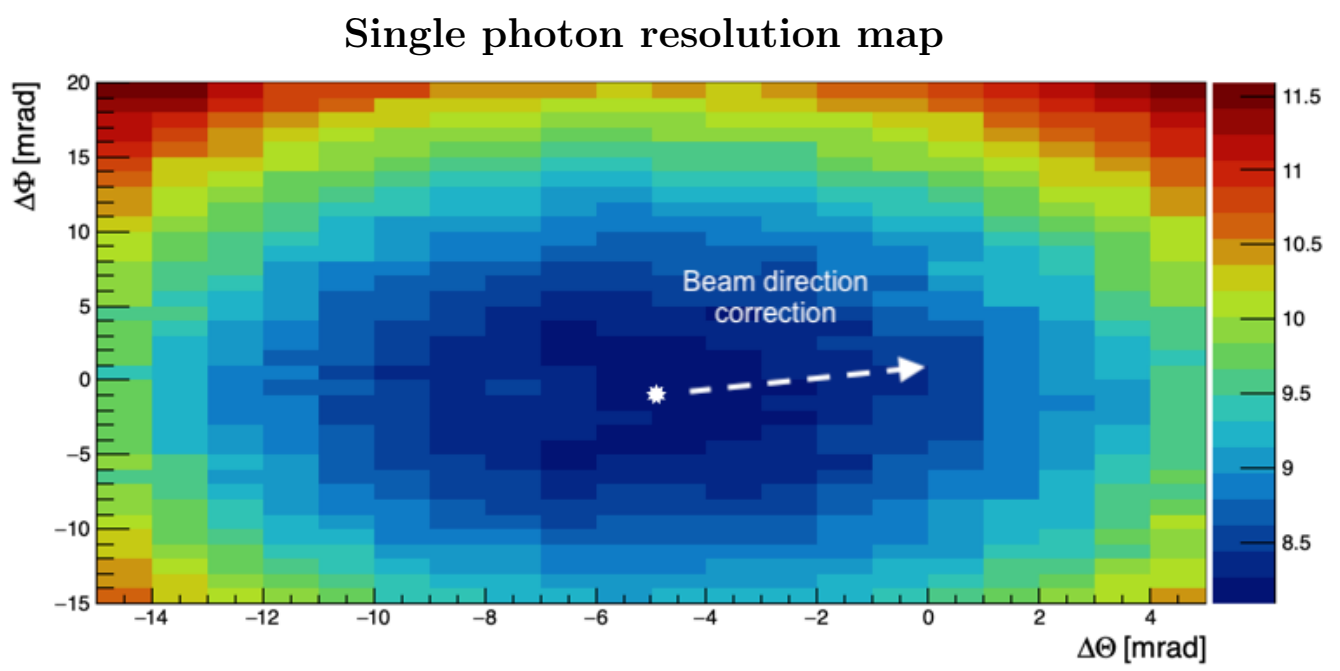

Figure 4.21: Example of the single photon resolution (SPR) map as a function of the charged track polar and azimuthal angles rotations at $20^{\circ}$ polar angle. The color scale corresponds to the SPR value using proton-tagged data.

After adding the beam direction correction

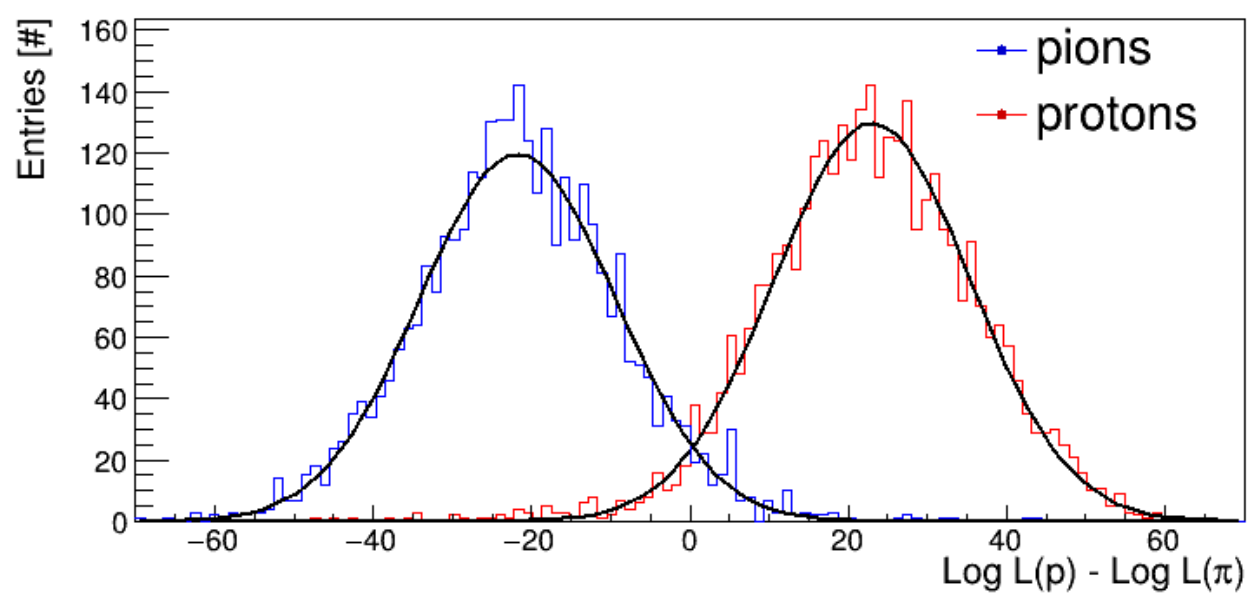

Figure 4.22: $\pi / \mathrm{p}$ log-likelihood difference distributions for proton-tagged (red) and pion-tagged (blue) beam events $(\approx 8000)$ as result of the geometrical reconstruction at $7 \mathrm{GeV} / \mathrm{c}$ momentum and $20^{\circ}$ polar angle. The $\pi / \mathrm{p}$ separation power value is $3.62 \pm 0.2$ standard deviations. 
Single MCP-PMT reconstructed Cherenkov angle shift

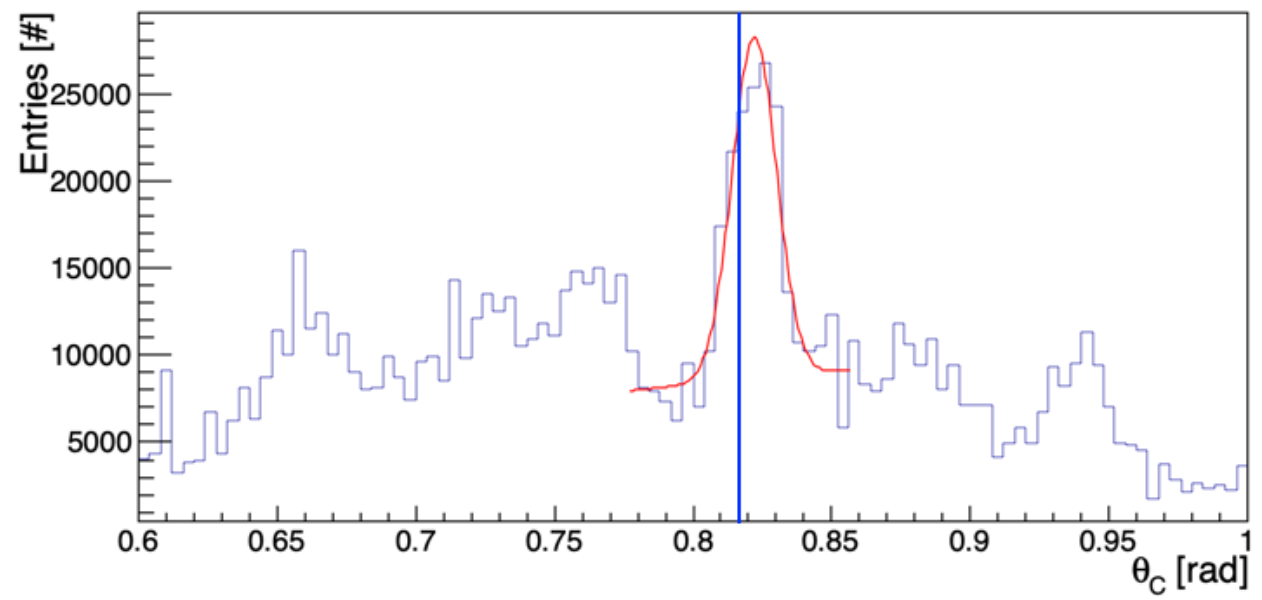

Figure 4.23: Example of the reconstructed Cherenkov angle for a single MCP-PMT using proton beam date at $20^{\circ}$ polar angle and $7 \mathrm{GeV} / c$ momentum. The blue line indicates the expected value of the Cherenkov angle. The reconstructed value is shifted by $\approx 5 \mathrm{mrad}$.

After adding the per MCP-PMT Cherenkov angle correction

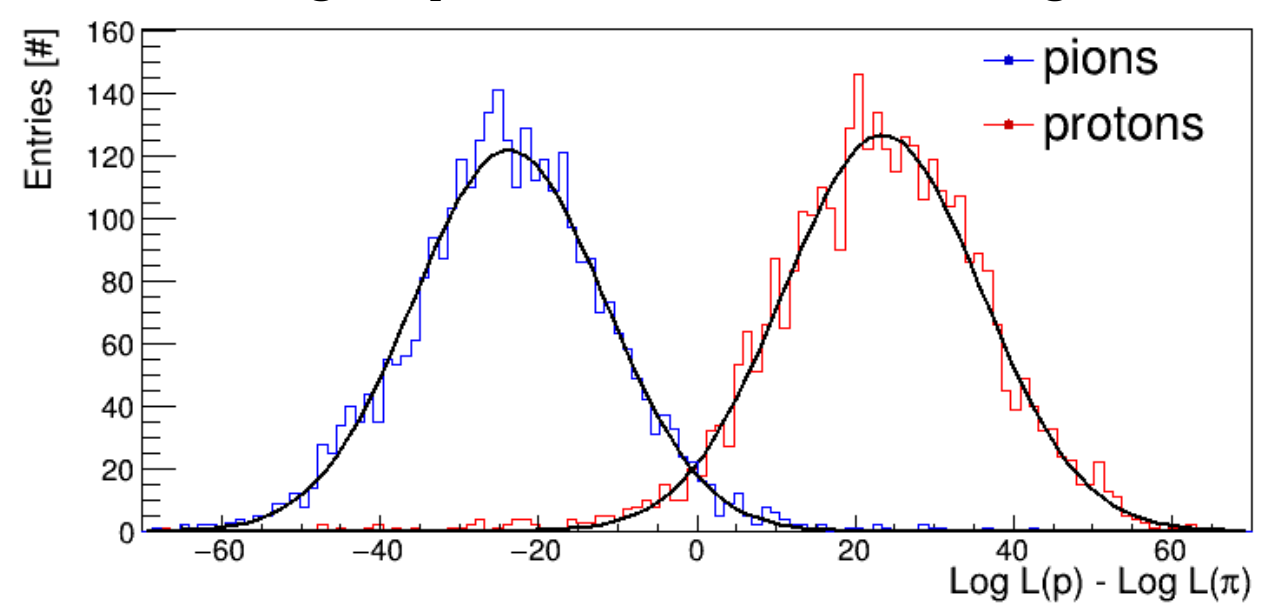

Figure 4.24: $\pi / \mathrm{p}$ log-likelihood difference distributions for proton-tagged (red) and pion-tagged (blue) beam events $(\approx 8000)$ as result of the geometrical reconstruction at $7 \mathrm{GeV} / \mathrm{c}$ momentum and $20^{\circ}$ polar angle. The $\pi / \mathrm{p}$ separation power value is $3.83 \pm 0.2$ standard deviations. 


\section{Chromatic Correction}

The Chromatic dispersion affects the Cherenkov angle $\theta_{C}(\lambda)$ at the production according to $\cos \theta_{C}(\lambda)=1 / \beta n(\lambda)$, where $n(\lambda)$ is refractive index for a wave length $\lambda$. In the meanwhile, the propagation time also depends on the wavelength based on the group velocity according to to $t_{\text {pro }}=d_{b a r} \times n_{g}(\lambda) / c_{o}$, Where $t_{\text {pro }}$ is the photon propagation time, $d_{b a r}$ is the photon path in the bar, and $n_{g}(\lambda)$ is the group refraction index. The photon wavelength can be determined by measuring the arrival time and compare it with the expected arrival time. Figure $4.25 \mathrm{a}$ shows an example of the difference between the measured and expected Cherenkov angle as a function of the difference between the measured and expected Cherenkov photons propagation time at $90^{\circ}$ polar angle. The Chromatic correction was applied by fitting a line to the correlation plot. Fig. 4.25a shows the correlation after applying the Chromatic correction. Fig. 4.26 shows the $\pi / p$ log-likelihood difference distributions after applying the correction to chromatic aberration, the $\pi / p$ separation is 4.18 standard deviations.
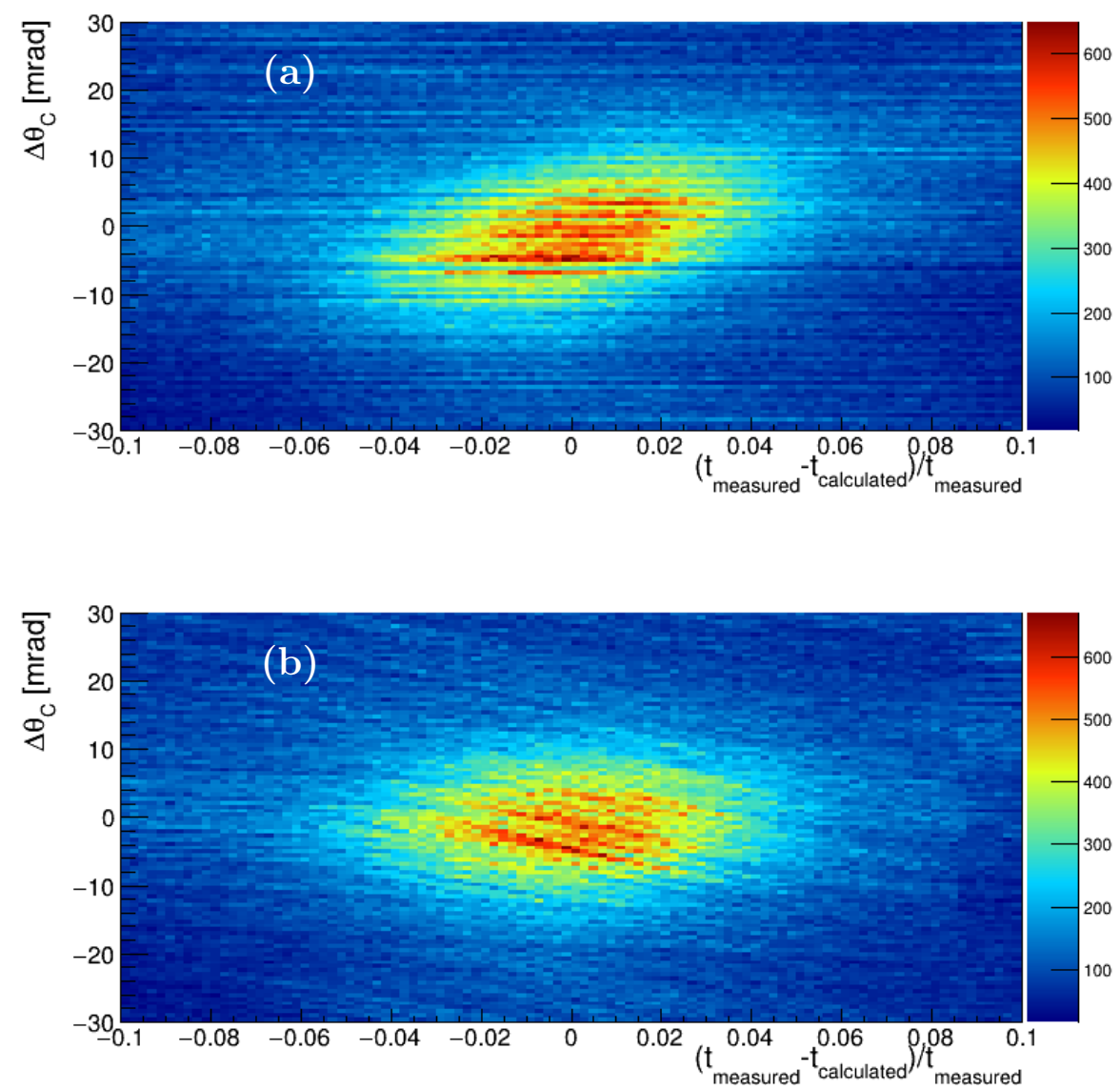

Figure 4.25: (a) Example of the reconstructed Cherenkov angle per photon from proton beam data at $7 \mathrm{GeV} / \mathrm{c}$ momentum and $90^{\circ}$ polar angle, (b) after the chromatic correction. 
After adding the chromatic correction

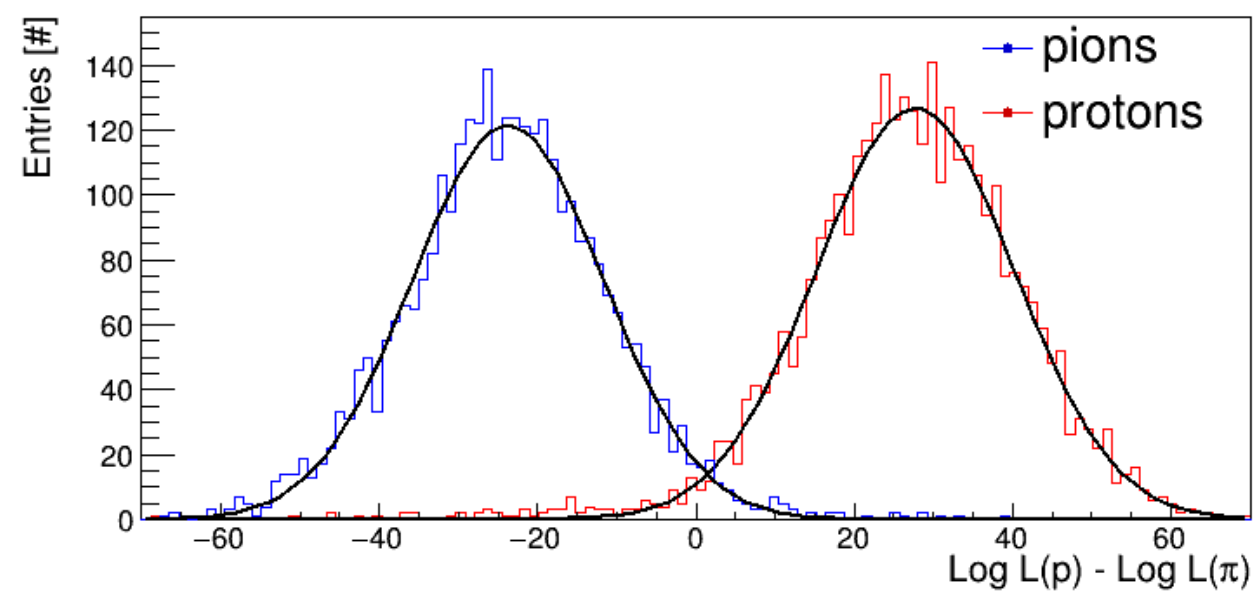

Figure 4.26: $\pi / \mathrm{p}$ log-likelihood difference distributions for proton-tagged (red) and pion-tagged (blue) beam events $(\approx 8000)$ as result of the geometrical reconstruction at $7 \mathrm{GeV} / \mathrm{c}$ momentum and $20^{\circ}$ polar angle. The $\pi / \mathrm{p}$ separation power value is $4.18 \pm 0.2$ standard deviations.

\subsubsection{Geometrical Reconstruction Second Approach}

The $\pi / p$ log-likelihood difference distributions using the second approach of the geometrical reconstruction are shown in Fig. 4.27. The $\pi / p$ separation is $4.0 \pm 0.2$ standard deviations. This result was obtained after applying all of the corrections described above. Since the performance of the first and second approaches are comparable to each other, the DIRC PID performance comparison between the data and simulation will be discussed in the following sub-sections using the first approach.

Geometrical reconstruction second approach

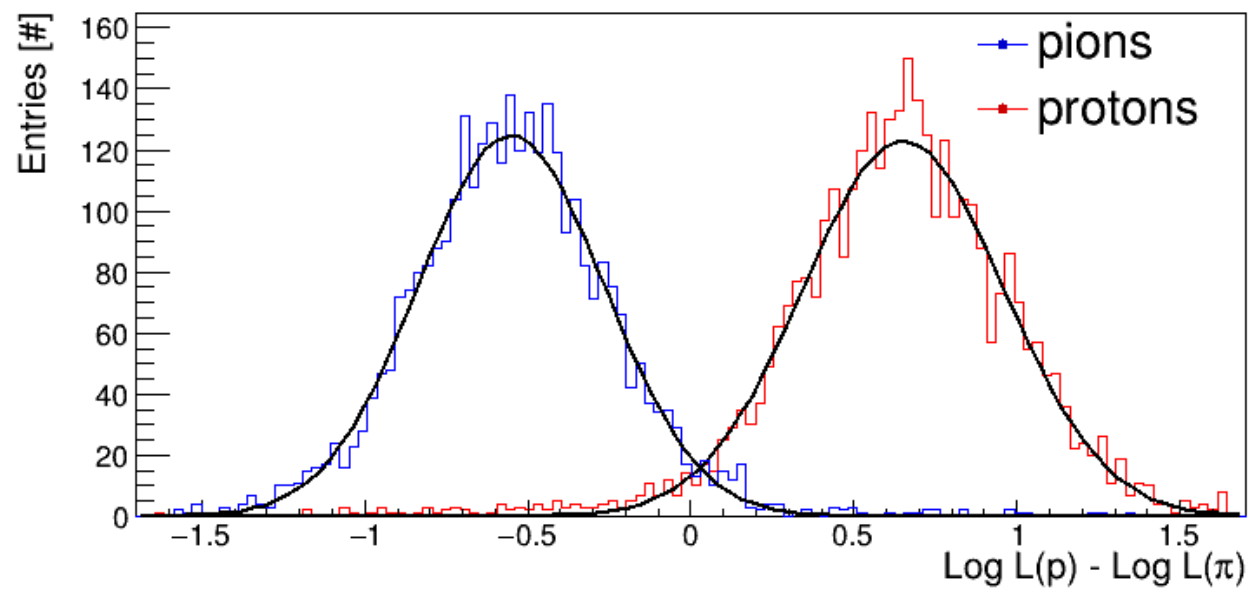

Figure 4.27: $\pi / \mathrm{p}$ log-likelihood difference distributions for proton-tagged (red) and pion-tagged (blue) beam events $(\approx 8000)$ as result of the geometrical reconstruction at $7 \mathrm{GeV} / \mathrm{c}$ momentum and $20^{\circ}$ polar angle. The $\pi / \mathrm{p}$ separation power value is $4.0 \pm 0.2$ standard deviations. 


\subsection{Error Calculations}

The following sections describe the errors that have an influence on the DIRC performance in terms of the statistical error, the photon hit time selection error, and the TOF PID quality error. Given that the estimated systematic errors are shown only for $20^{\circ}$ polar angle and the error values are different for other polar angles.

\subsubsection{Separation Power}

\section{Statistical Error}

Statistically independent samples were used to calculate the separation power. Figure 4.28 shows the separation power as a function of the statistical sample number. The estimated statistical error is \pm 0.07 s.d. corresponds to \pm RMS of the separation power distribution from all statistical samples. The red lines define the statistical error.

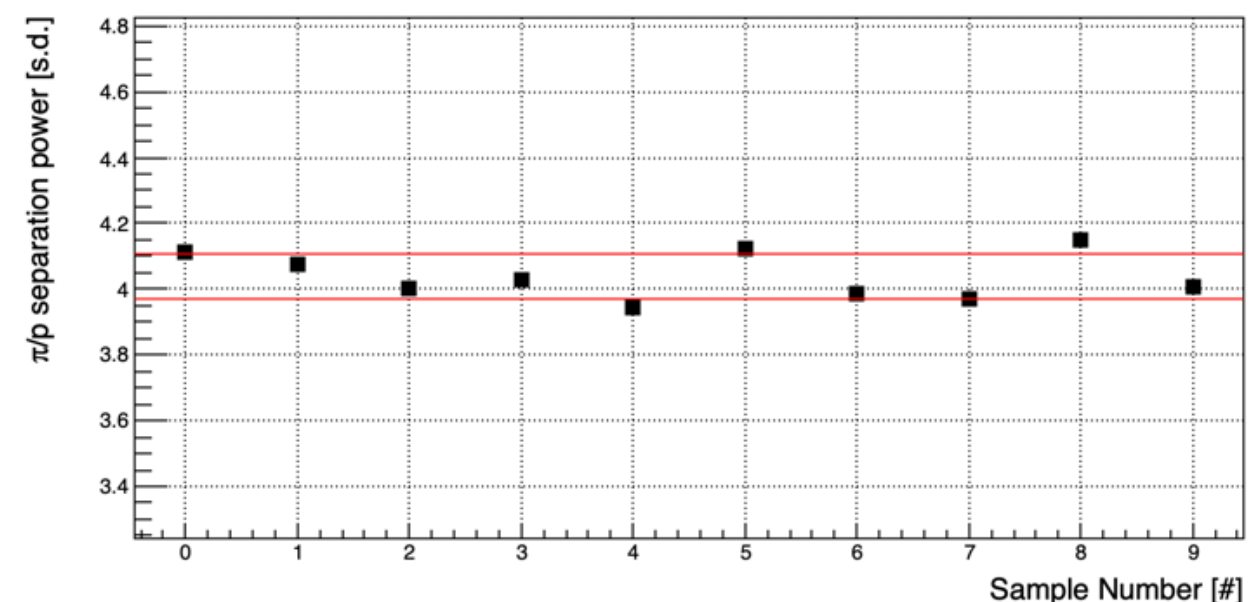

Figure 4.28: Pion/proton separation power as a function of the statistical sample number, using beam data at $7 \mathrm{GeV} / \mathrm{c}$ momentum and $20^{\circ}$ polar angle. The red lines show the statistical error contribution.

\section{Time Cut Error}

The hit time selection cut is used to reduce the noise and suppress the reconstruction ambiguities. The following equation describeds the time selection criteria:

$$
|\Delta T|<0.04 t+b
$$

Where $\Delta \mathrm{T}$ is the time difference between the measured time and the calculated time, $t$ is the calculated photon solution path time, the slope of the line was tuned to compensate for the chromatic dispersion, and $\mathrm{b}$ is the time cut parameter.

The effect of three different values of the time cut parameter on the acceptance time window and the reconstruction ambiguities can be seen in Fig. 4.29. By increasing the time cut parameter, the reconstruction ambiguities increases. Figure 4.30 shows the impact of increasing the time window acceptance on the separation power. To 
some extent, increasing the time window has a positive impact on the separation power. However, after further increment, the separation power starts to decrease due to increasing the combinatorial background. The projection of the separation power values of Fig. 4.30 on the y-axis is shown in Fig 4.31. The estimated contribution of the $\Delta \mathrm{T}$ cut error is \pm 0.1 s.d. corresponds to \pm RMS of the separation power distribution from all of the time cut parameter values.

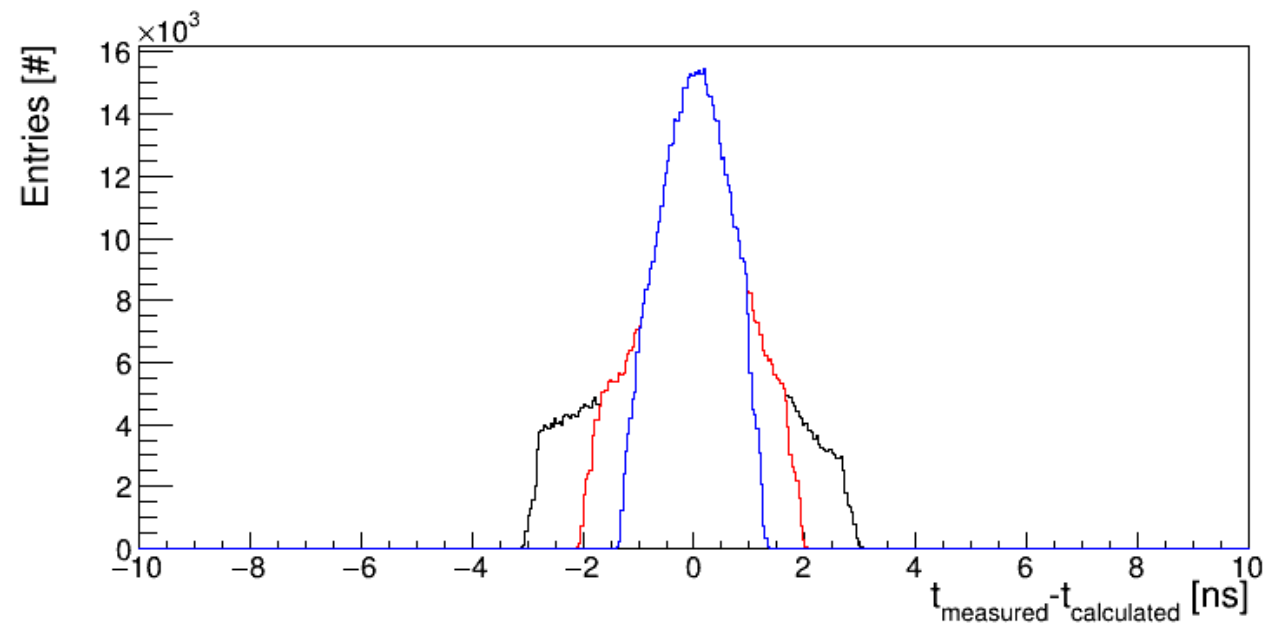

Figure 4.29: Impact of the time cut parameter on the reconstruction ambiguities for $\mathrm{b}=0$ (blue), $\mathrm{b}=1.2$ (red), and $\mathrm{b}=2.2$ (black)

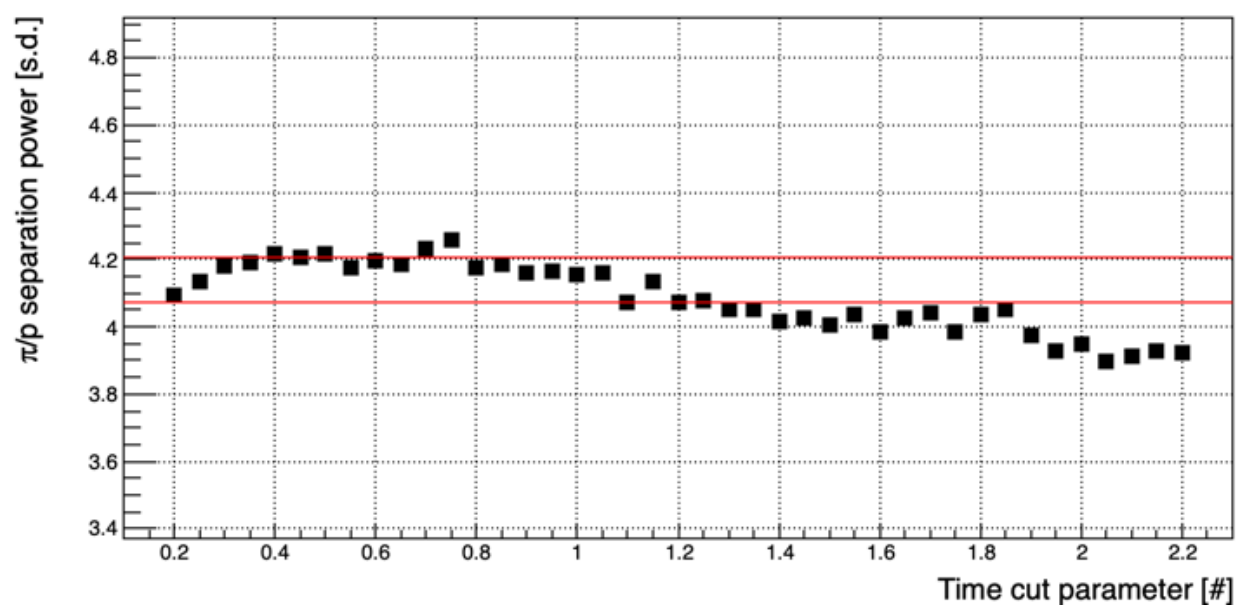

Figure 4.30: Pion/proton separation power as a function of the time cut parameter, using beam data at $7 \mathrm{GeV} / \mathrm{c}$ momentum and $20^{\circ}$ polar angle. The red lines show the statistical error contribution.

\section{External PID Error}

The prototype performance was evaluated using external PID. The quality of the external PID influences the DIRC performance outcomes. To estimate this effect, the time difference distribution between TOF1 and TOF2 was sub-divided into slices as shown in Fig. 4.32. The TOF slice 1 has the lowest purity because it is close to the contaminated region between the proton and pion peaks, while TOF slice 9 has 


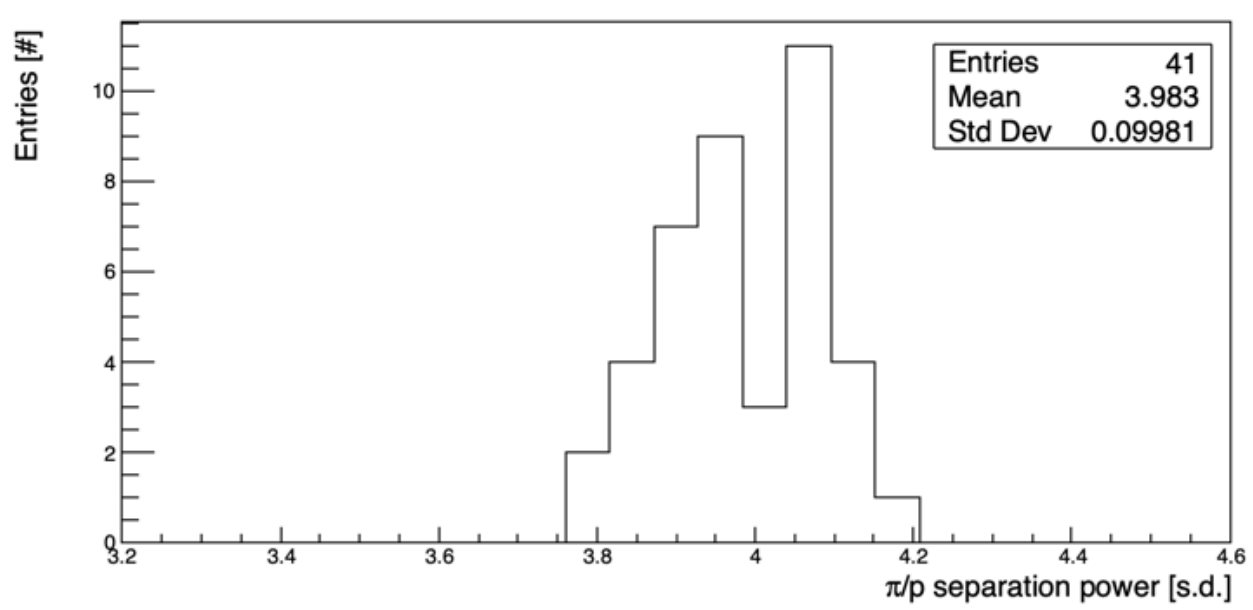

Figure 4.31: The projection of the separation power values of Fig. 4.30 on the y-axis

the best purity.

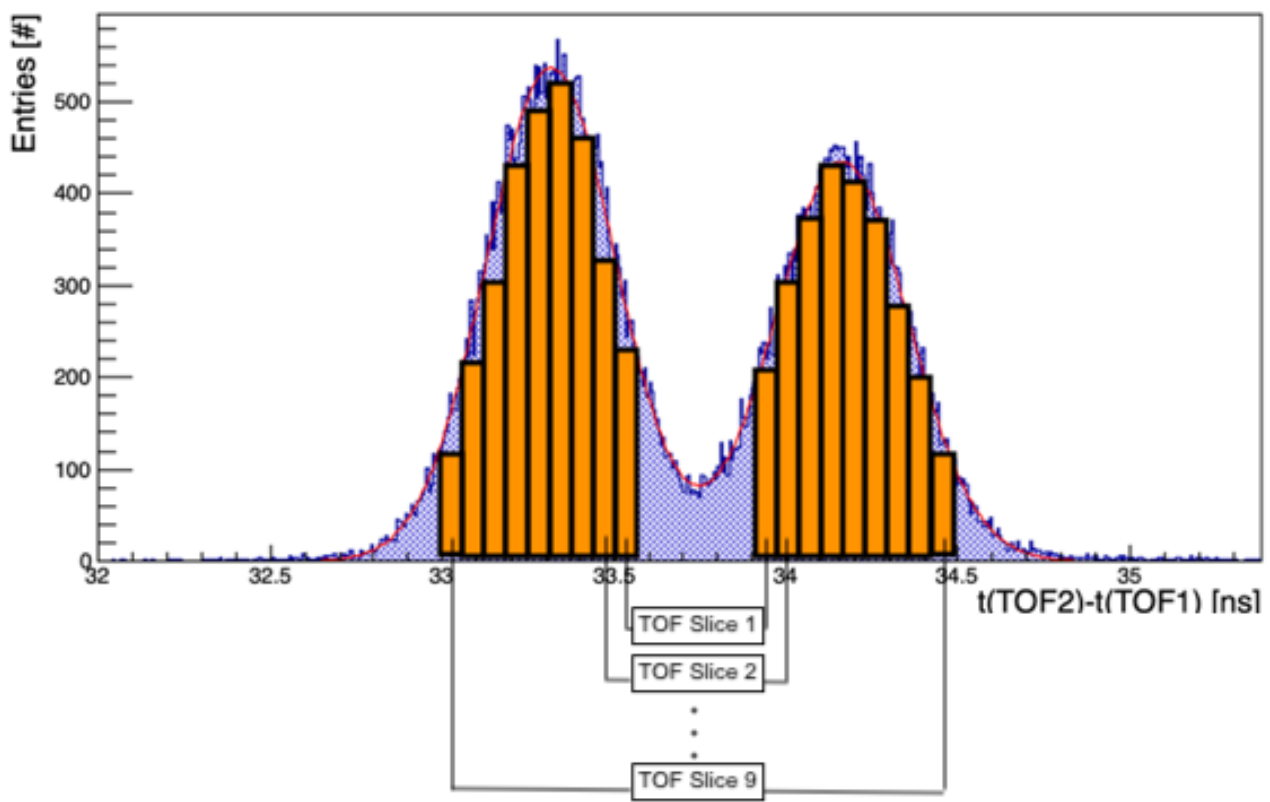

Figure 4.32: Schematic drawing of the TOF1 and TOF2 slicing.

The purity of the external PID samples influences the separation power. Figure 4.33 show the impact of selecting different TOF slices on the PID performance. The separation power increased by increasing the sample purity. The first four TOF slices were removed from PID performance analyses. Therefore, those four slices were removed from the error calculations as well. The estimated error contribution of the external PID quality is \pm 0.16 s.d. corresponds to \pm RMS of the separation power distribution from all of the TOF slices. 


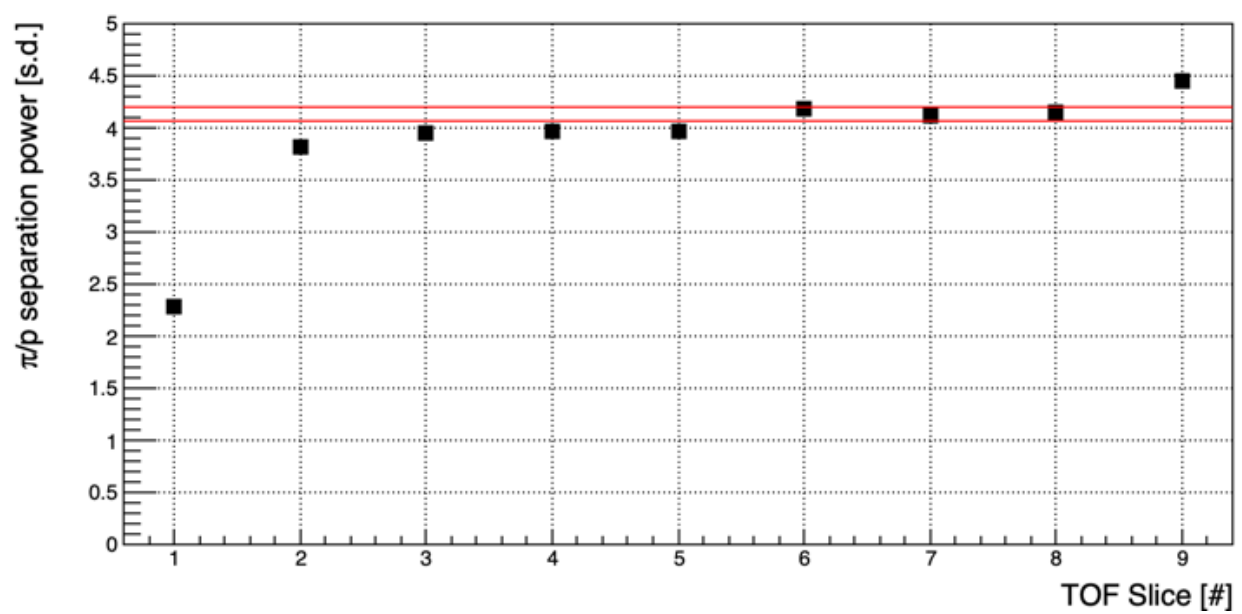

Figure 4.33: Pion/proton separation power as a function of the TOF slice number, using beam data at $7 \mathrm{GeV} / \mathrm{c}$ momentum and $20^{\circ}$ polar angle. The red lines show the statistical error contribution.

\section{Total Error of the Separation Power}

The total error $\sigma_{\text {total }}$ can be approximated as the following:

$$
\sigma_{\text {total }}=\sqrt{\sigma_{\text {stat }}^{2}+\sigma_{\Delta T}^{2}+\sigma_{T O F}^{2}}
$$

where $\sigma_{\text {stat }}$ is the statistical error contribution, $\sigma_{\Delta T}$ is track hit time selection error contribution and $\sigma_{T O F}$ is the TOF error contribution.

The total error for the separation power is mainly influenced by the quality of external PID samples and the $\Delta \mathrm{T}$ cut, with about \pm 0.2 standard deviations.

\subsubsection{Single Photon Resolution}

\section{Statistical Error}

The statistical error of the single photon resolution is \pm 0.05 mrad. Figure 4.34 shows the single photon resolution as a function of the statistical sample number. The statistical error was estimated the same way as described in section 4.8.1.

\section{Time Cut Error}

The single photon resolution is sensitive to the time cut. Figure 4.35 shows that increasing the acceptance time window leads to accumulating the reconstruction combinatorial background under the Cherenkov angle peak, which increases the single photon resolution. The calculated error of the hit time selection is \pm 0.2 mrad it was estimated the same way as described in section 4.8.1.

\section{External PID Error}

The purity of the external PID samples has an important impact on the single photon resolution. Figure 4.36 show the effect of selecting different TOF slices on the single photon resolution. The single photon resolution decreased by increasing 


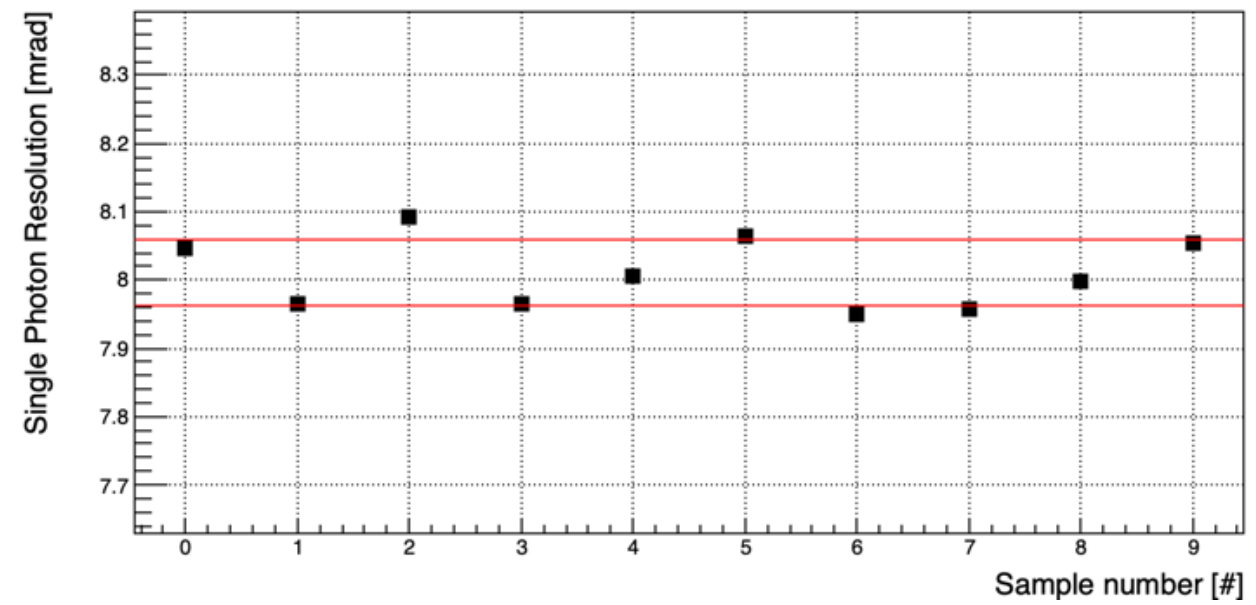

Figure 4.34: Single photon resolution for protons as a function of the statistical sample number, using beam data at $7 \mathrm{GeV} / \mathrm{c}$ momentum and $20^{\circ}$ polar angle. The red lines show the statistical error contribution.

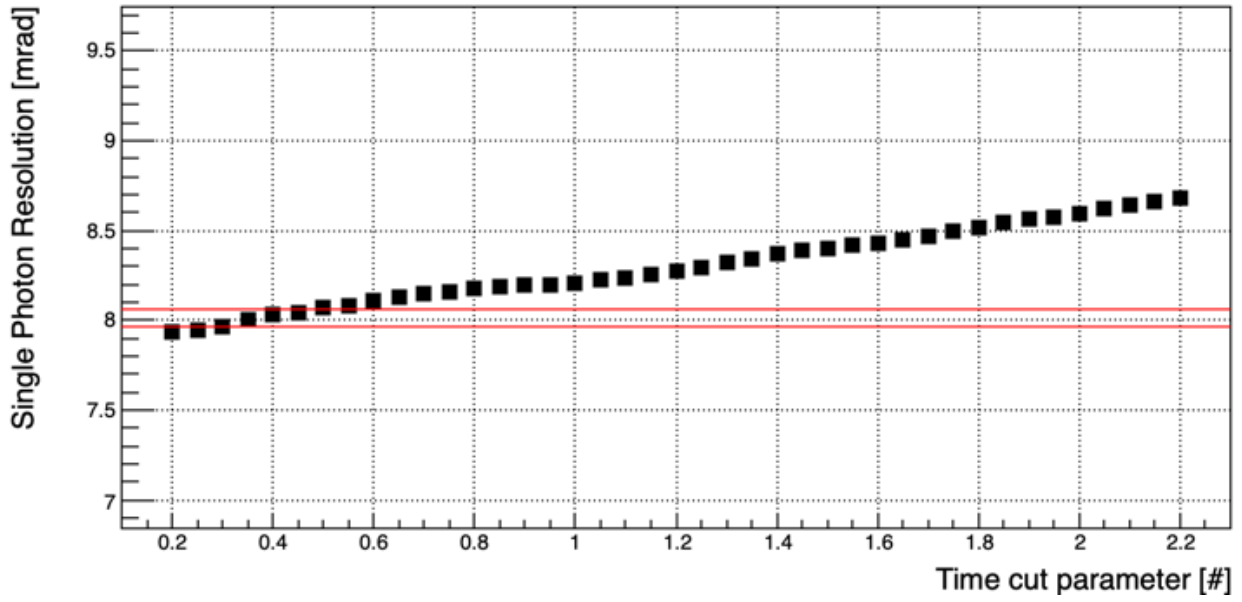

Figure 4.35: Single photon resolution for protons as a function of the time cut parameter, using beam data at $7 \mathrm{GeV} / \mathrm{c}$ momentum and $20^{\circ}$ polar angle. The red lines show the statistical error contribution. 
the sample purity. The first four slices were removed from the error calculations, as they were removed from the analyses because they have the lowest purity. The estimated error contribution of the external PID quality is $\pm 0.15 \mathrm{mrad}$. Corresponds to \pm RMS of the single photon resolution distribution from all of the TOF slices, as described in section 4.8.1.

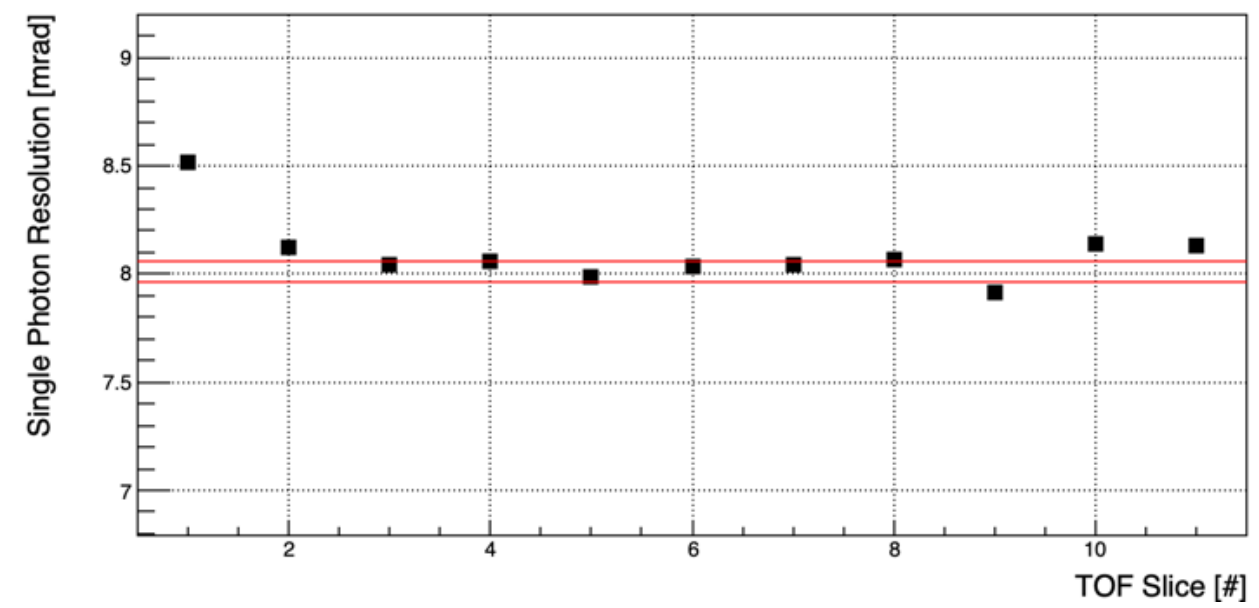

Figure 4.36: Single photon resolution for proton as a function of the TOF slice number, using beam data at $7 \mathrm{GeV} / \mathrm{c}$ momentum and $20^{\circ}$ polar angle. The red lines show the statistical error contribution.

\section{Total Error of the Single Photon Resolution}

The total error for the single photon resolution is about $\pm 0.25 \mathrm{mrad}$, affected by the external PID sample purity and the $\Delta \mathrm{T}$ cut.

\subsubsection{Photon Yield}

\section{Statistical Error}

Figure 4.37 shows the photon yield per track as a function of the statistical sample number. The calculated photon yield statistical error is \pm 0.2 photon, and it was estimated the same way as described in section 4.8.1.

\section{Time Cut Error}

Figure 4.38 shows the photon yield per track as a function of the time cut parameter. Increasing the time acceptance leads to an increase in the probability of counting more photons. The photon yield statistical error is \pm 4 photon, and it was calculated the same way as described in section 4.8.1.

\section{External PID Error}

Figure 4.39 show the impact of selecting different TOF slices on the photon yield. The external PID TOF slicing has an effect on the photon yield with about \pm 1 photon, corresponds to \pm RMS of the photon yield distribution from all of the TOF slices. 


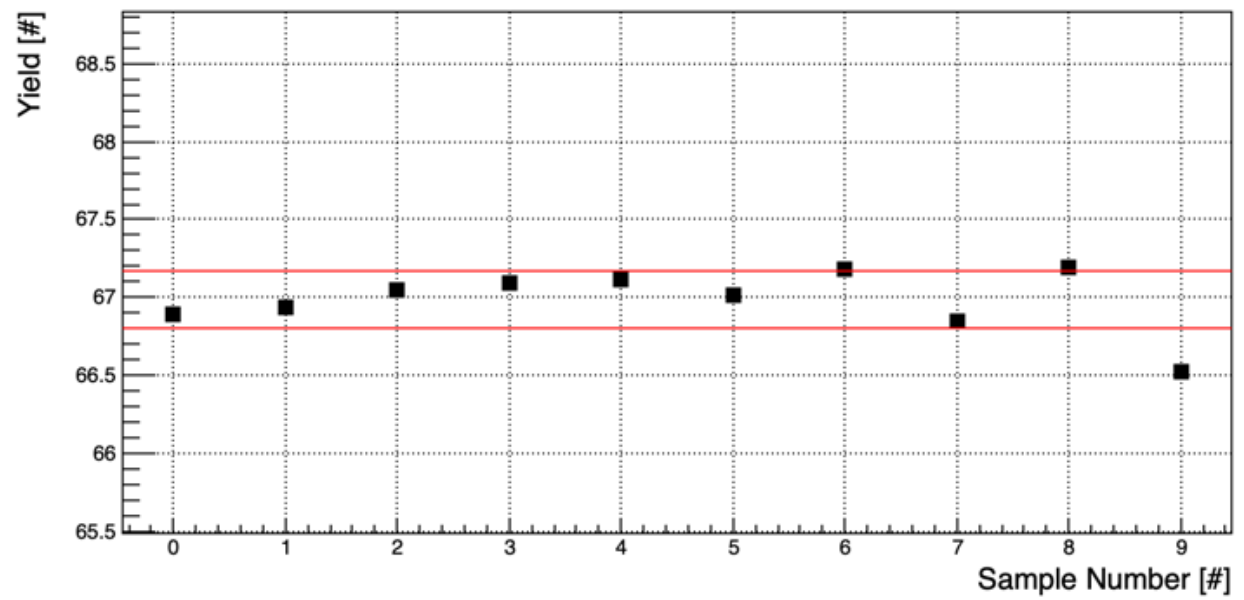

Figure 4.37: The photon yield as a function of the statistical sample number, using beam data at $7 \mathrm{GeV} / \mathrm{c}$ momentum and $20^{\circ}$ polar angle. The red lines show the statistical error contribution.

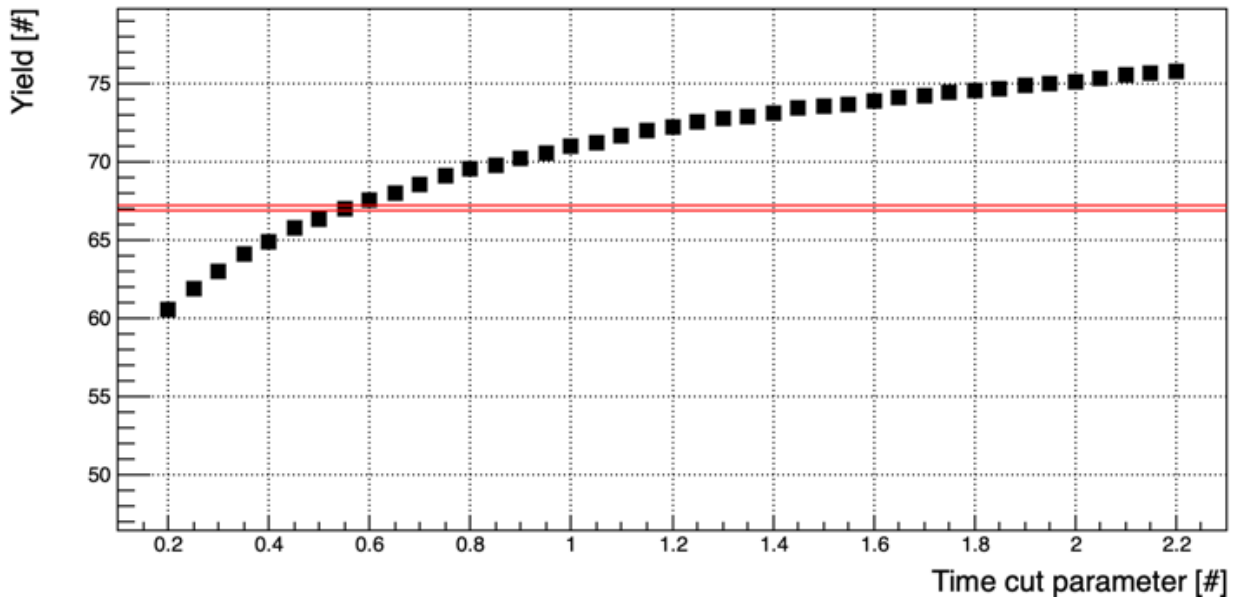

Figure 4.38: The photon yield as a function of the time cut parameter, using beam data at $7 \mathrm{GeV} / \mathrm{c}$ momentum and $20^{\circ}$ polar angle. The red lines show the statistical error contribution. 


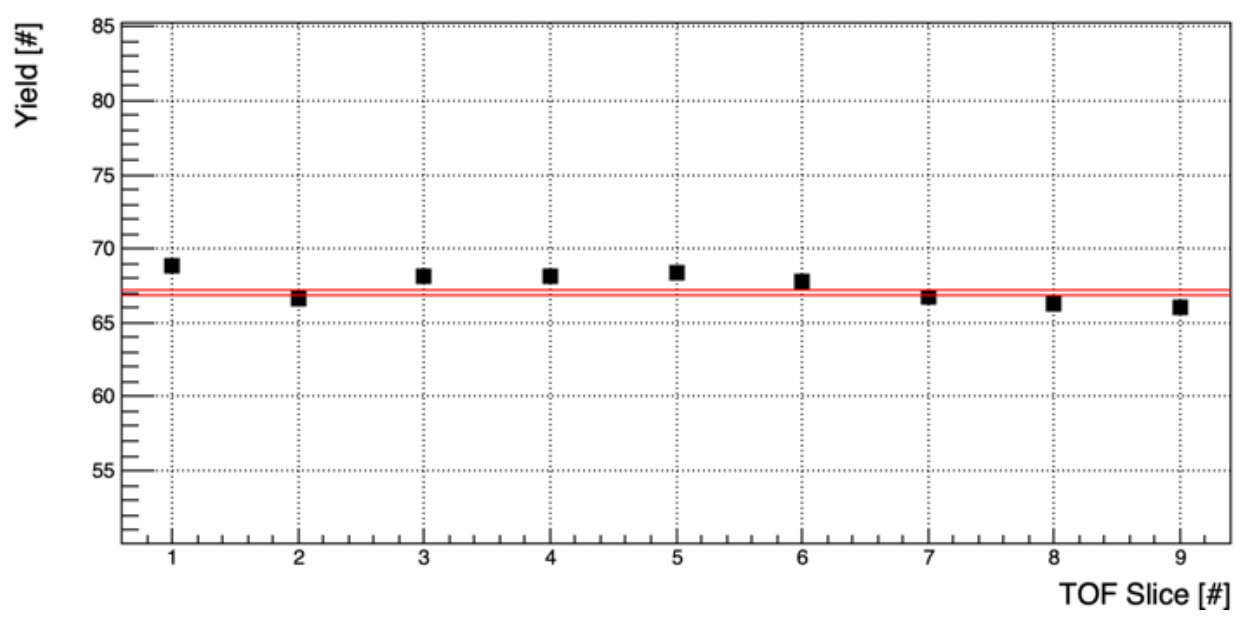

Figure 4.39: Photon yield as a function of the TOF slice number, using beam data at $7 \mathrm{GeV} / \mathrm{c}$ momentum and $20^{\circ}$ polar angle. The red lines show the statistical error contribution.

\section{Total Error of the Photon Yield}

The total error of the photon yield is significantly dominated by the $\Delta \mathrm{T}$ cut with about \pm 4 photon.

\subsubsection{Comparison Between Beam Data and Simulation}

Figure 4.40 shows the photon yield as a function of the prototype polar angle for both beam data and simulation. Good agreement between beam data and simulation is observed. The errors are mainly related to the statistical error. The photon yield is proportional to the length of the particle trajectory inside the radiator bar, which is smallest at the perpendicular incidence and increases towards the end of the bars. In addition, the fraction of photons trapped by total internal reflection generally rises with larger values of $|\cos \theta|$, except close to $90^{\circ}$ where a fraction of both direct and reflected photons can be trapped, leading to an enhancement of trapping efficiency [46].

The single photon Cherenkov angle resolution (SPR) as a function of the beam polar angle for tagged protons with $7 \mathrm{GeV} / \mathrm{c}$ momentum is shown in Fig. 4.41. The result shows good agreement of the SPR with simulation. The errors are dominated by the external PID sample purity and the hit selection time cut.

The separation power from the log-likelihood difference distributions for protontagged and pion-tagged beam events is illustrated in Fig. 4.42 as a function of the polar angle for simulation and beam data. The distributions roughly follow the typical shape of the photon yield in the Barrel DIRC. The results show good agreement between beam data and simulations, and the errors are influenced by the statistical error, the quality of the external and the hit selection time cut. 


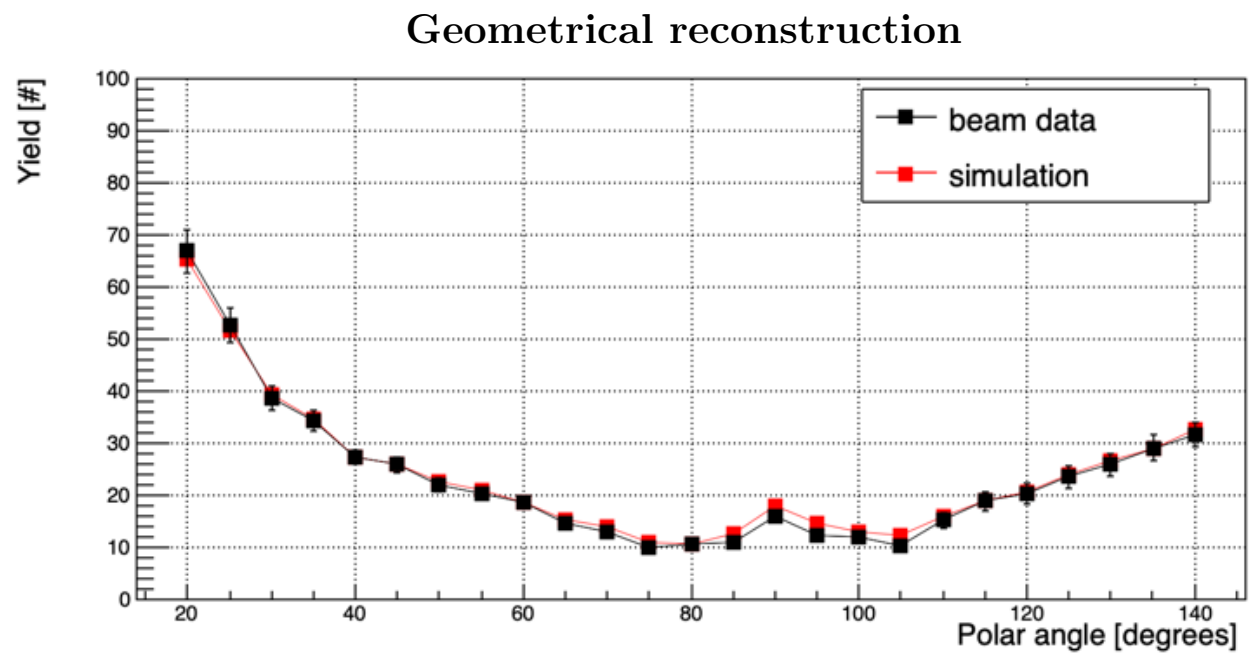

Figure 4.40: Photon yield as a function of the beam polar angle for tagged protons with $7 \mathrm{GeV} / \mathrm{c}$ momentum for beam data (black) and Geant4 simulation (red).

\section{Geometrical reconstruction}

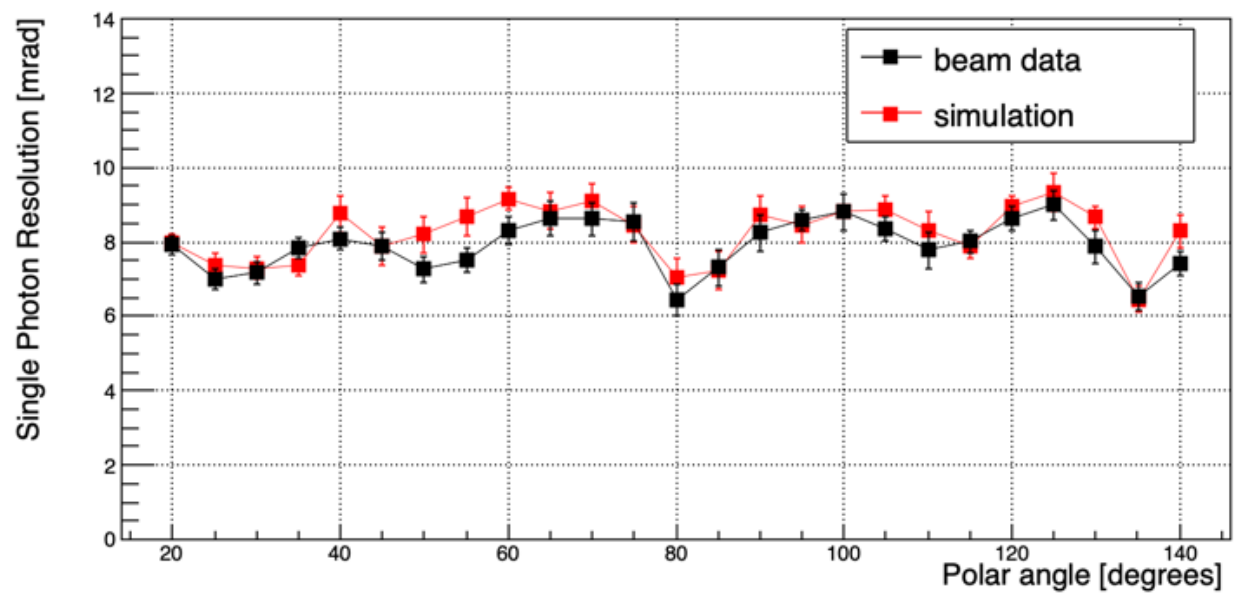

Figure 4.41: Cherenkov angle resolution per photon (SPR) as a function of the beam polar angle for tagged protons with $7 \mathrm{GeV} / \mathrm{c}$ momentum for beam data (black) and Geant4 simulation (red). 


\section{Geometrical reconstruction}

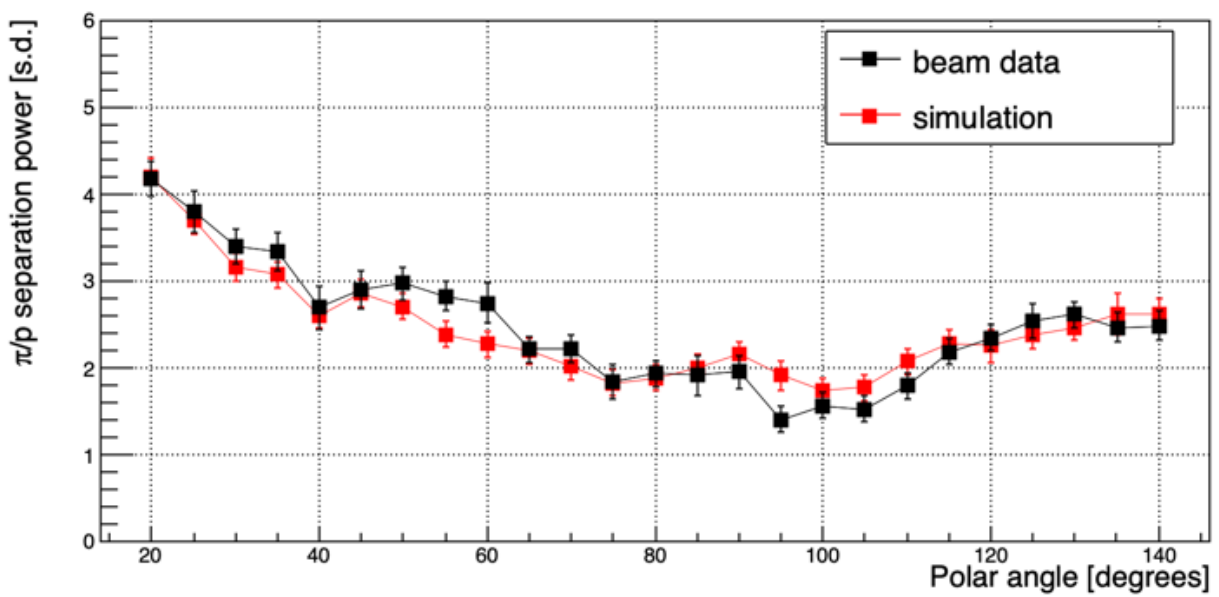

Figure 4.42: $\pi$ /p separation power from geometrical reconstruction as a function of the polar angle at $7 \mathrm{GeV} / \mathrm{c}$ momentum for beam data (black) and Geant4 simulation (red).

\subsubsection{Cherenkov Angle Data PDF}

This method is an extension of the geometric reconstruction. The basic concept is that the Cherenkov angle per photon is compared to the expected Cherenkov angle distributions for every pixel and every particle hypothesis, yielding the PID likelihoods. A large number of tracks with a given momentum and polar angle were used to produce the expected Cherenkov angle distributions per photon for protons and pions. These distributions were recorded for every pixel and stored in an array of normalized histograms, creating probability density functions (PDF). For a given track, the observed Cherenkov angle per photon for each hit pixel is compared to the histogram array to calculate the Cherenkov angle PDF-based likelihood for the photons to originate from a certain particle hypothesis. The data PDFs were taken from a statistically independent sample with the same beam conditions and detector configuration. In this study, 10000 proton and 10000 pion events were used to generate the PDFs for every corresponding polar angle. Figure 4.43 shows examples of the Cherenkov angle PDF for one pixel for proton (red) and pion (blue) tracks at 7 $\mathrm{GeV} / \mathrm{c}$ and $20^{\circ}$ polar angle. The vertical lines show the expected Cherenkov angles for protons and pions.

The separation power from the proton-pion log-likelihood difference distributions for proton-tagged and pion-tagged beam events with $7 \mathrm{GeV} / \mathrm{c}$ at $20^{\circ}$ polar angle is shown in Fig. 4.44. The achieved separation power is $4.3 \pm 0.2$ standard deviations. Figure 4.45 shows the $\pi / \mathrm{p}$ separation power as a function of the polar angle at $7 \mathrm{GeV} / \mathrm{c}$ momentum for beam data for three different reconstruction approaches: the standard geometrical reconstruction without corrections, the standard geometrical reconstruction after applying the corrections, and the Cherenkov angle data PDF approach. The standard geometrical reconstruction improved for all polar angles after the corrections were applied. The performance of the Cherenkov angle data $\mathrm{PDF}$ is close to the fully corrected standard geometrical construction method.

The main advantage of using the Cherenkov angle data PDF is that it is much less 


\section{Cherenkov angle data PDF}

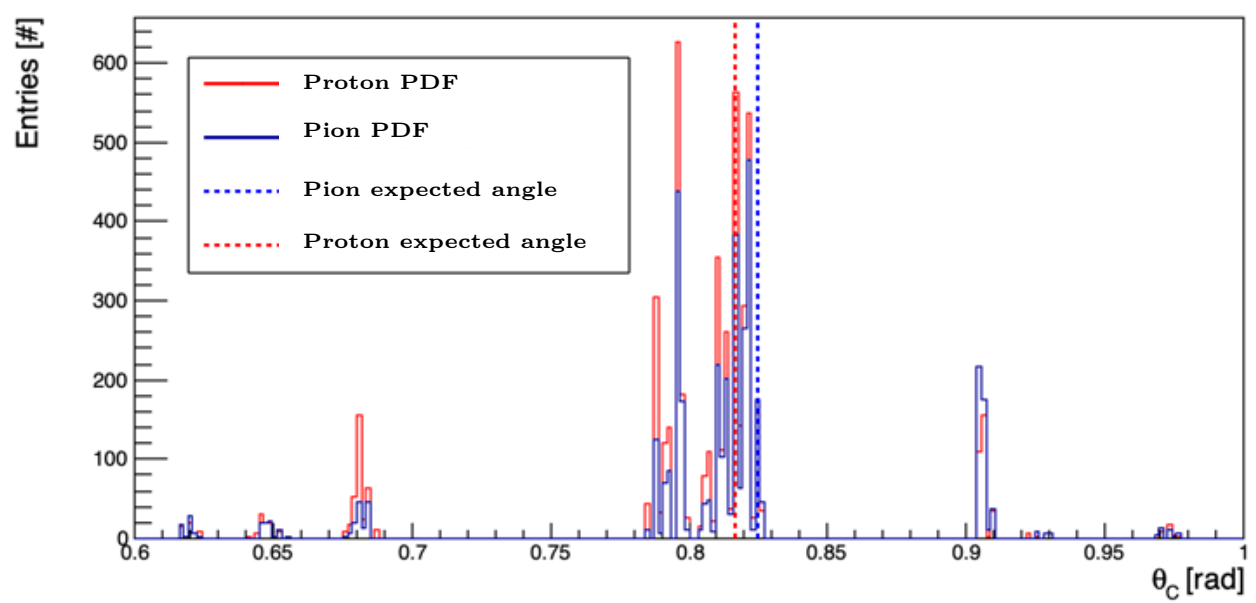

Figure 4.43: Example of protons (red) and pions (blue) PDFs taken from beam data at $7 \mathrm{GeV} / \mathrm{c}$ momentum and a polar angle of at $20^{\circ}$ for one pixel. The vertical dashed lines indicate the expected Cherenkov angle.

\section{Cherenkov angle data PDF}

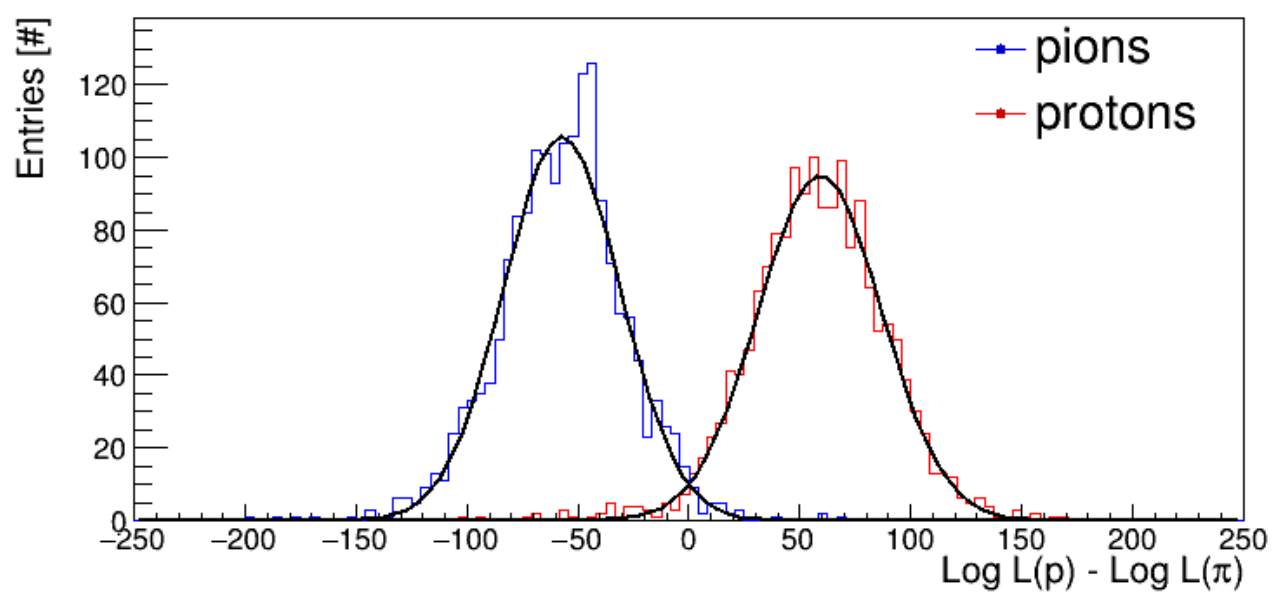

Figure 4.44: $\pi / \mathrm{p}$ log-likelihood difference distributions for proton-tagged (red) and pion-tagged (blue) beam events as result of the Cherenkov data PDF approach at $7 \mathrm{GeV} / \mathrm{c}$ momentum and a $20^{\circ}$ polar angle. The $\pi / \mathrm{p}$ separation power extracted from the Gaussian fits is 4.3 standard deviations. 
dependent on proper alignment and calibration of the data, and no additional correction is required as the detector misalignment effect was minimized. The Cherenkov angle PDF method shows comparable results for $\pi / \mathrm{p}$ separation power to the standard geometrical reconstruction after several corrections procedures. The disadvantage is that a large number of tracks are required for every track direction to create the PDFs. While this method works well for a beam test, it creates significant challenges for collider experiment data.

\section{Cherenkov angle data PDF}

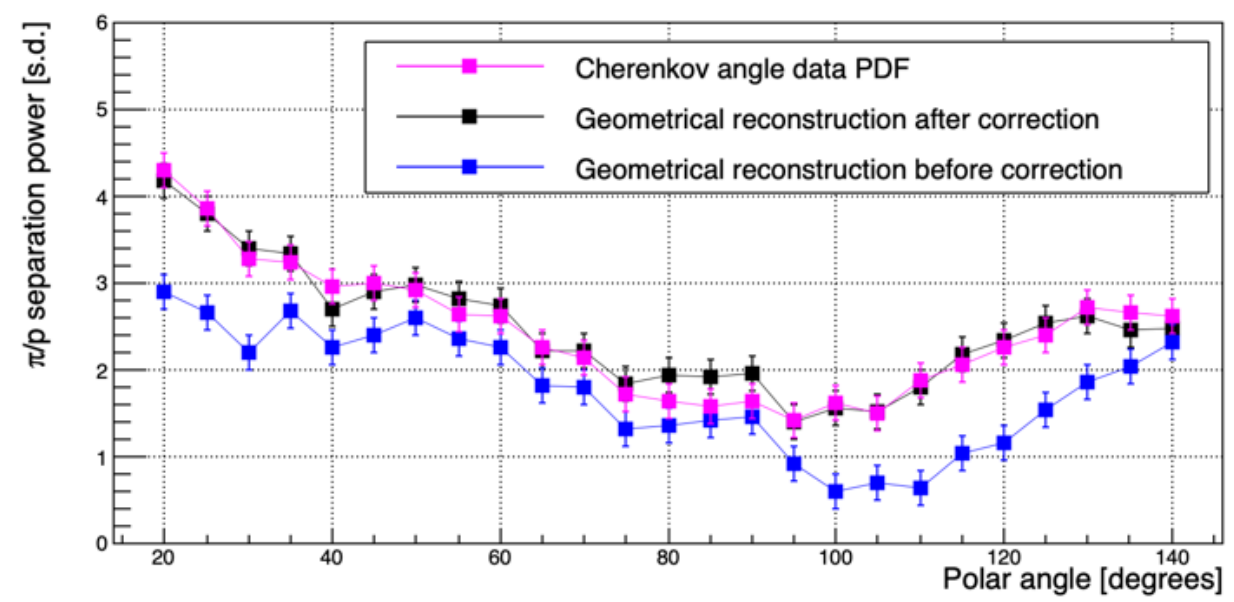

Figure 4.45: $\pi / \mathrm{p}$ separation power as a function of the polar angle at $7 \mathrm{GeV} / \mathrm{c}$ momentum for beam data using: the geometrical reconstruction without corrections (blue), the geometrical reconstruction after applying corrections (black), and the Cherenkov angle data PDF approach (magenta).

\subsubsection{Time-based Imaging}

A detailed discussion of the time-based imaging PID broach is described in section 3.4.2. The beam data PDFs are taken from a statistically independent sample with the same beam condition and detector configuration, separated by the TOF tags. Figure 4.46 shows the log-likelihood difference for proton and pion hypotheses for a sample with $7 \mathrm{GeV} / \mathrm{c}$ protons and pions at $20^{\circ}$ polar angle. The calculated $\pi / \mathrm{p}$ separation power is 4.96 standard deviations.

Figure 4.47 shows the $\pi / p$ separation power with $7 \mathrm{GeV} / \mathrm{c}$ momentum as a function of the polar angle. The resultant PID performance is in good agreement with the expectation from simulations. The main advantage of this reconstruction approach is that the performance is found to be superior to the geometrical reconstruction results. The disadvantages of the implementation using PDFs based on beam data or simulation are the large number of events required to create the PDFs and the large amount of computer memory required to store those PDFs. 


\section{Time-based imaging}

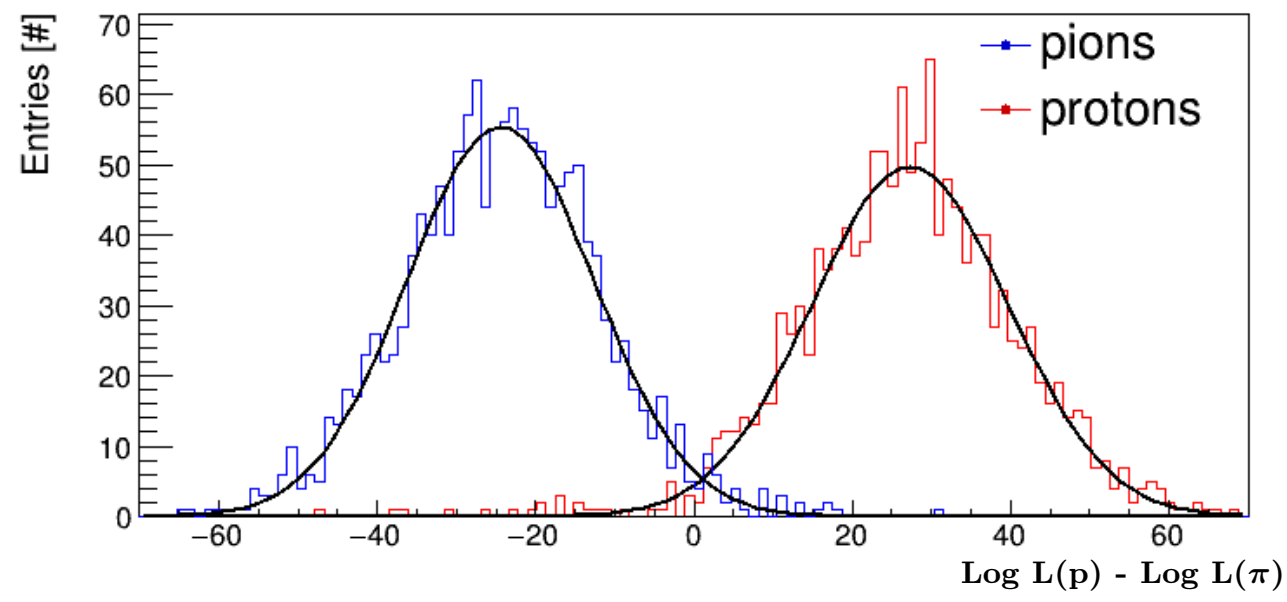

Figure 4.46: $\pi / \mathrm{p}$ log-likelihood difference distributions for proton-tagged (red) and pion-tagged (blue) beam events as a result of the time-based imaging reconstruction at $7 \mathrm{GeV} / \mathrm{c}$ momentum and a $20^{\circ}$ polar angle. The $\pi / \mathrm{p}$ separation power extracted from the Gaussian fits is 4.96 standard deviations.

\section{Time-based imaging}

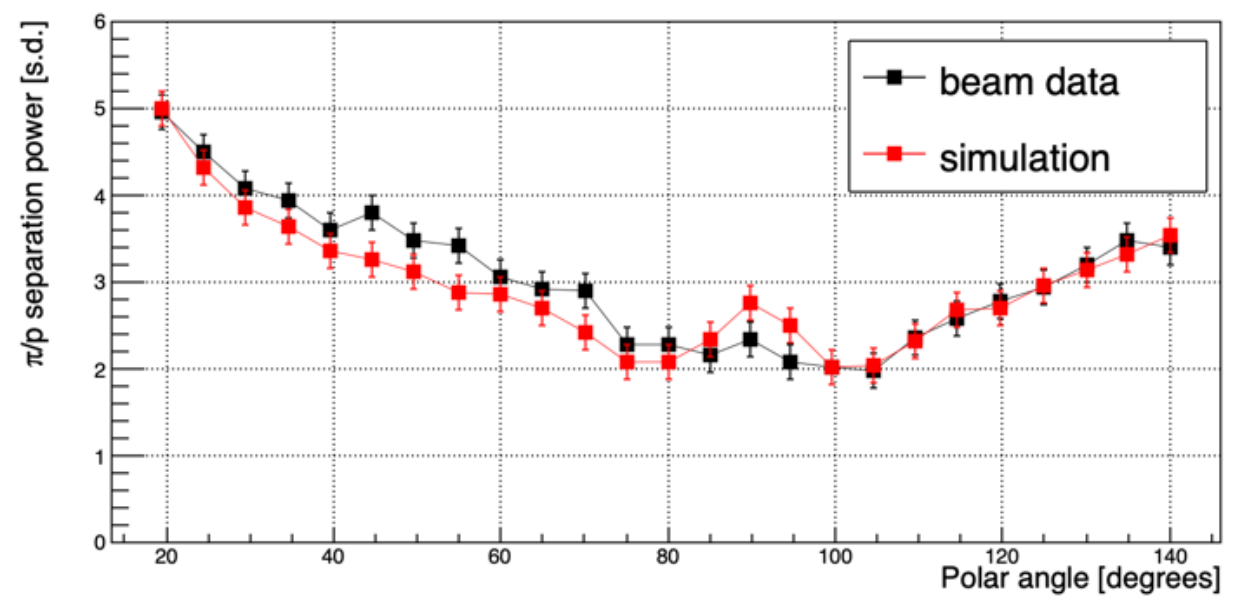

Figure 4.47: $\pi / \mathrm{p}$ separation power from time-based imaging as a function of the polar angle at $7 \mathrm{GeV} / \mathrm{c}$ momentum the beam data (black) and the simulation (red)[35]. 


\subsection{Azimuthal Angle Study}

The results described above show the performance of the PID and the reconstruction algorithms for the prototype under one particular condition, which requires that the charged track has a perpendicular azimuthal angle incidence on the DIRC bar. The typical azimuthal angle on PANDA is in the range of \pm 15 degrees due to the curvature of the track inside the magnetic field and the size of the azimuthal angle range of the bar box. Therefore, the prototype was designed to cover this azimuthal angle range to study how the azimuthal angle changes can influence the PID quality. Figure 4.48 shows the prototype azimuthal angle at 15 degrees azimuthal angle. The prototype rotation was remote-controlled and monitored by a camera.

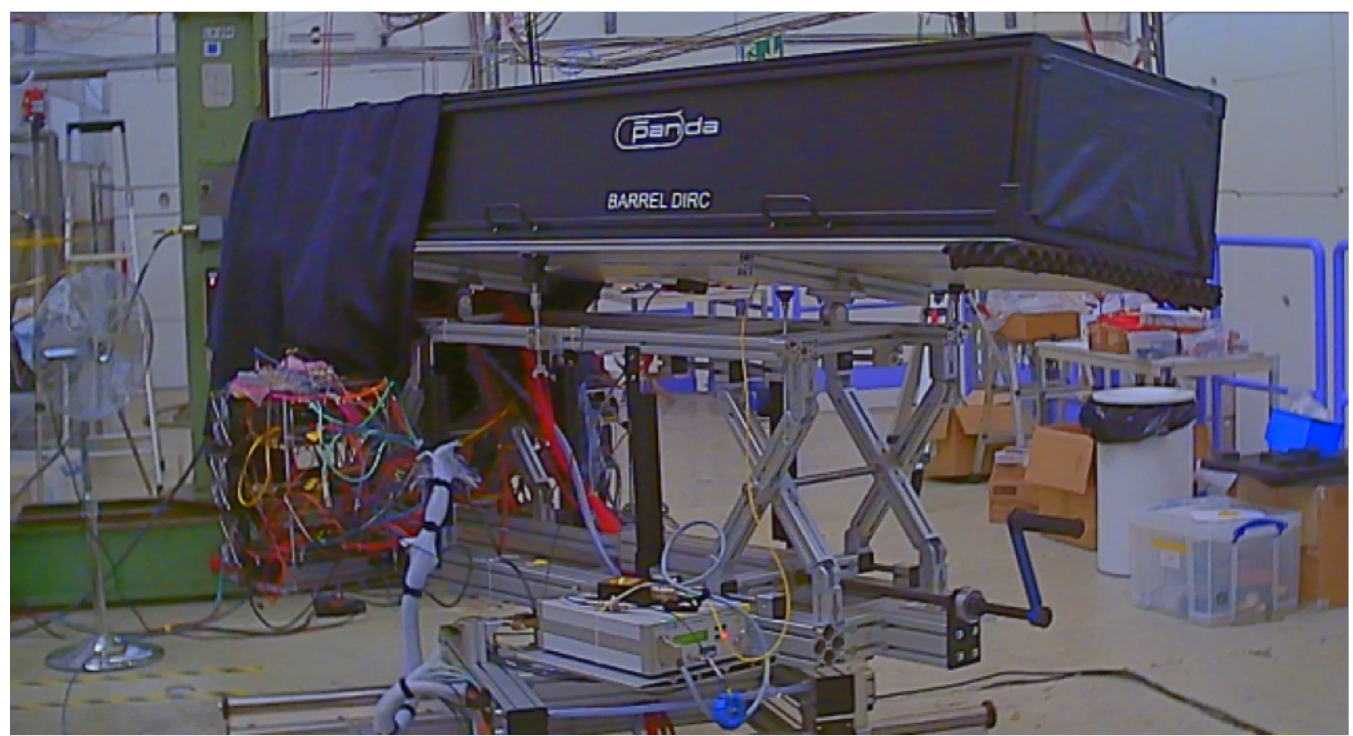

Figure 4.48: Photograph of the prototype rotated by 15 degree azimuthal angle.

Cherenkov photons are produced uniformly along the charged particle track in the bar. Changing the azimuthal angle causes the Cherenkov ring to degenerate and complicate the hit pattern on the MCP-PMT plane. Example of the hit pattern at $20^{\circ}$ polar angle and $10^{\circ}$ azimuthal angle is shown in Fig. 4.49.

The photon yield was evaluated for several azimuthal angles as shown in Fig. 4.51, the obtained results of the beam data are consistent with simulations. The photon yield shows the familiar shape of increasing the yield for steeper angles, due to a longer track length within the bar and the bump near $90^{\circ}$ polar angle due to both sides of the Cherenkov ring being totally internally reflected. The total internal reflection efficiency decreased by increasing the azimuthal angle, and this causes additional photon yield loss. This effect is represented in the simulation as well. It was reported during the visual inspection of the setup at T9, that the optical coupling in the glue joint between the bar and the lens shows a big snowflake-like structure. It looks as if the air was pulled into the glue joint, as shown in Fig. 4.50.

The $\pi / p$ separation power of the beam data as a result of Cherenkov angle data PDF and the time imaging is compared to Geant4 simulation in Fig. 4.52 and Fig. 4.53, for different azimuth angles. The $\pi / \mathrm{p}$ separation power in unite of standard deviations, 
(a) Beam Data
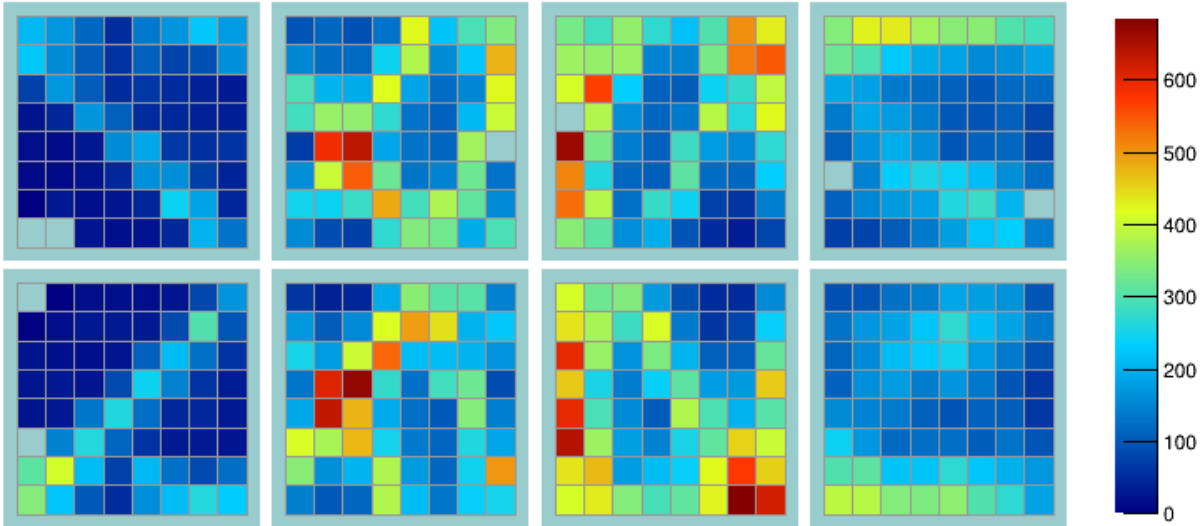

\section{(b) Geant4}
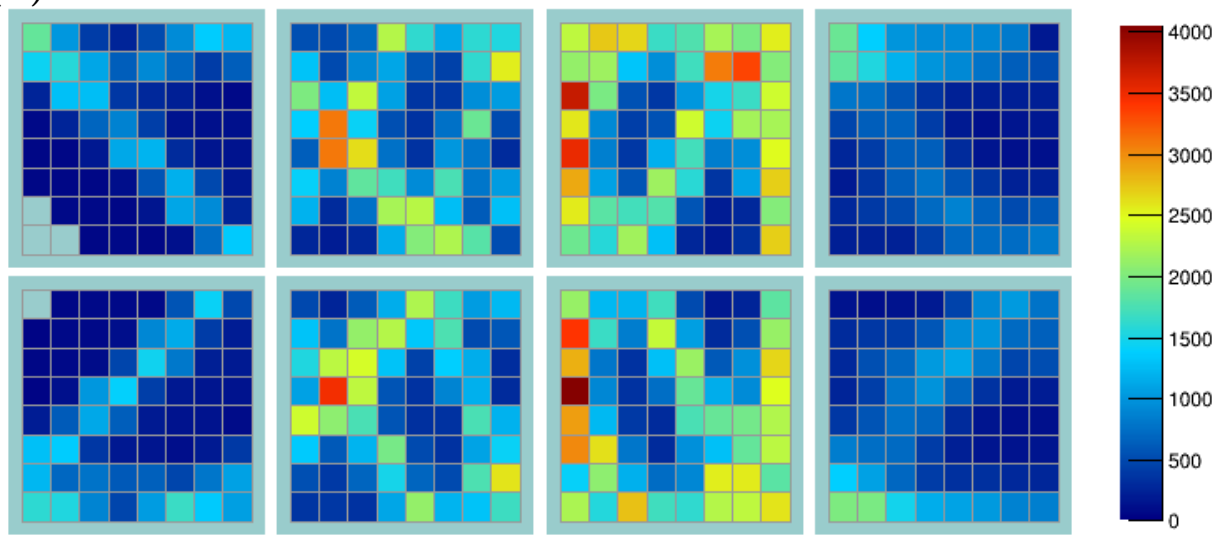

Figure 4.49: Example of the hit pattern at $20^{\circ}$ polar angle and $10^{\circ}$ azimuthal angle for (a) beam data and (b) Geant4 simulation.

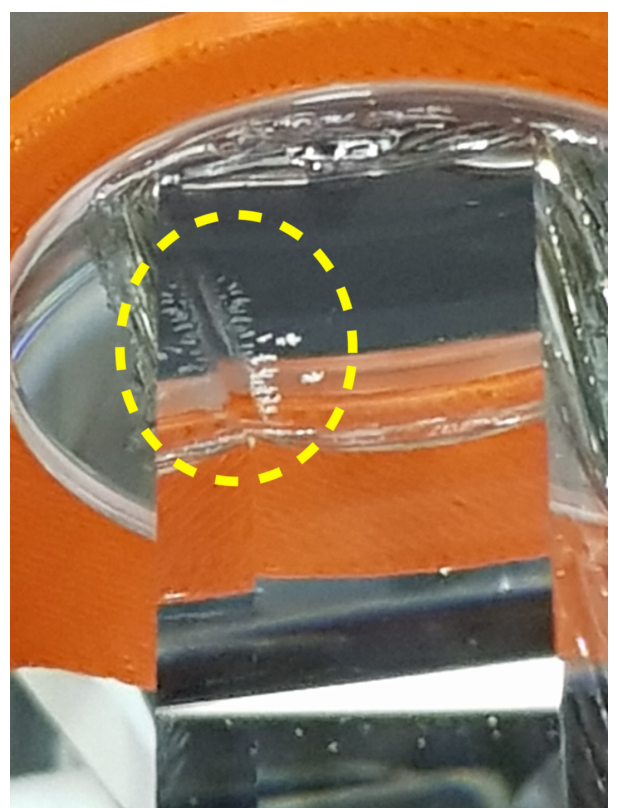

Figure 4.50: A snow flake-like structure can be seen on the bar-lens joint. 

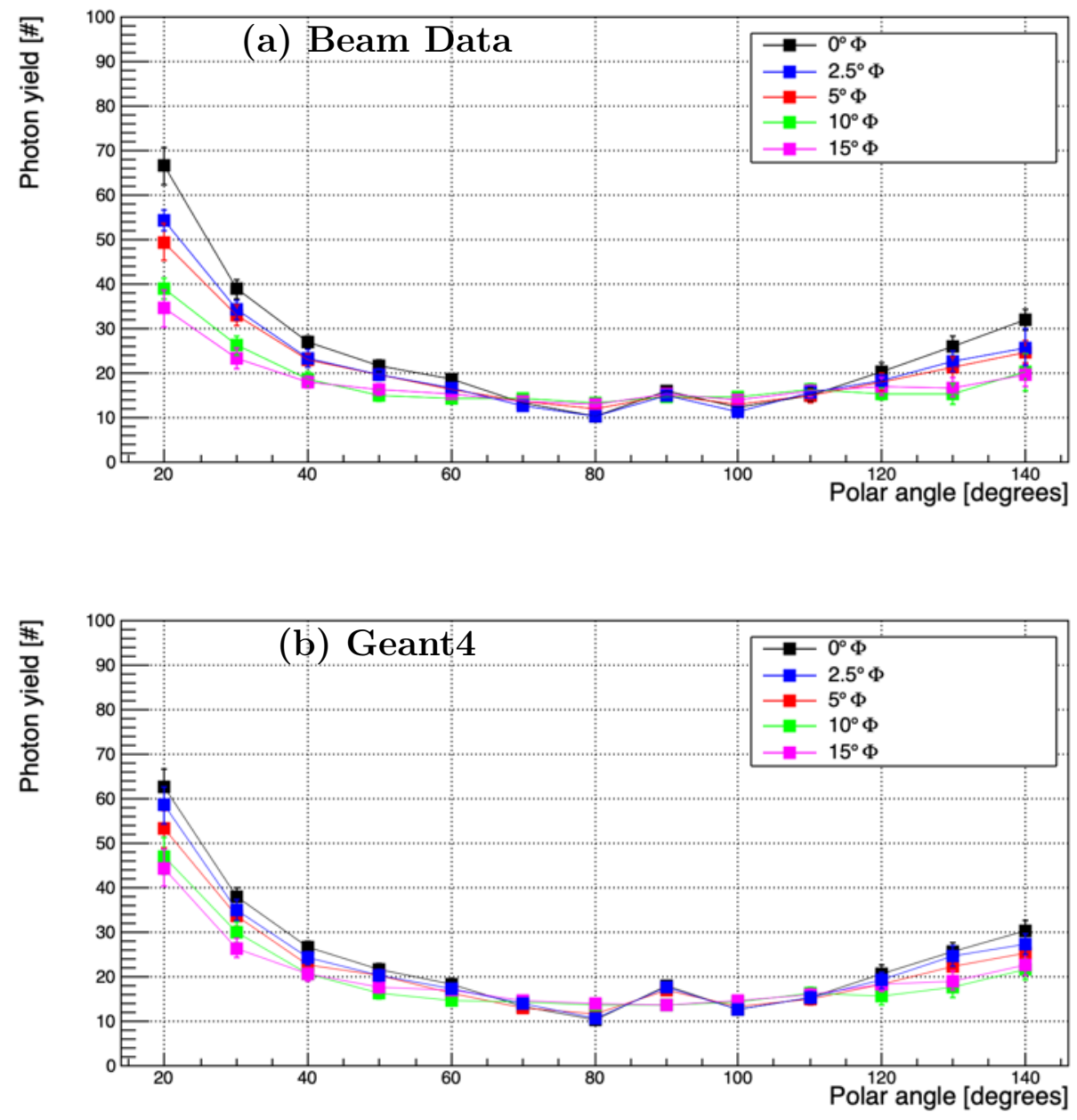

Figure 4.51: Photon yield as a function of the prototype polar angle for protons with $7 \mathrm{GeV} / \mathrm{c}$ momentum at different values of the azimuthal angles $\Phi$ as a result of geometrical reconstruction for beam data (a) and Geant4 simulation (b). 
shows the familiar shape described above. Given that the defect on the optical coupling during the azimuthal angle runs had influenced the PID quality. However, the azimuthal angles data points at 20 degree polar angle either exceed or close to 3 standard deviations using the Cherenkov angle data PDF method, and all of them exceed 3 s.d. using the time imaging PID method. The separation power decreases by increasing the azimuthal angle. This is because once the azimuthal angle incidence is no longer perpendicular, the ambiguities increase by a factor of two, which can represent a challenge for the PID and the reconstruction methods. However, the reconstruction and PID methods are robust under the azimuthal angle changes, delivering the PID requirements for PANDA.
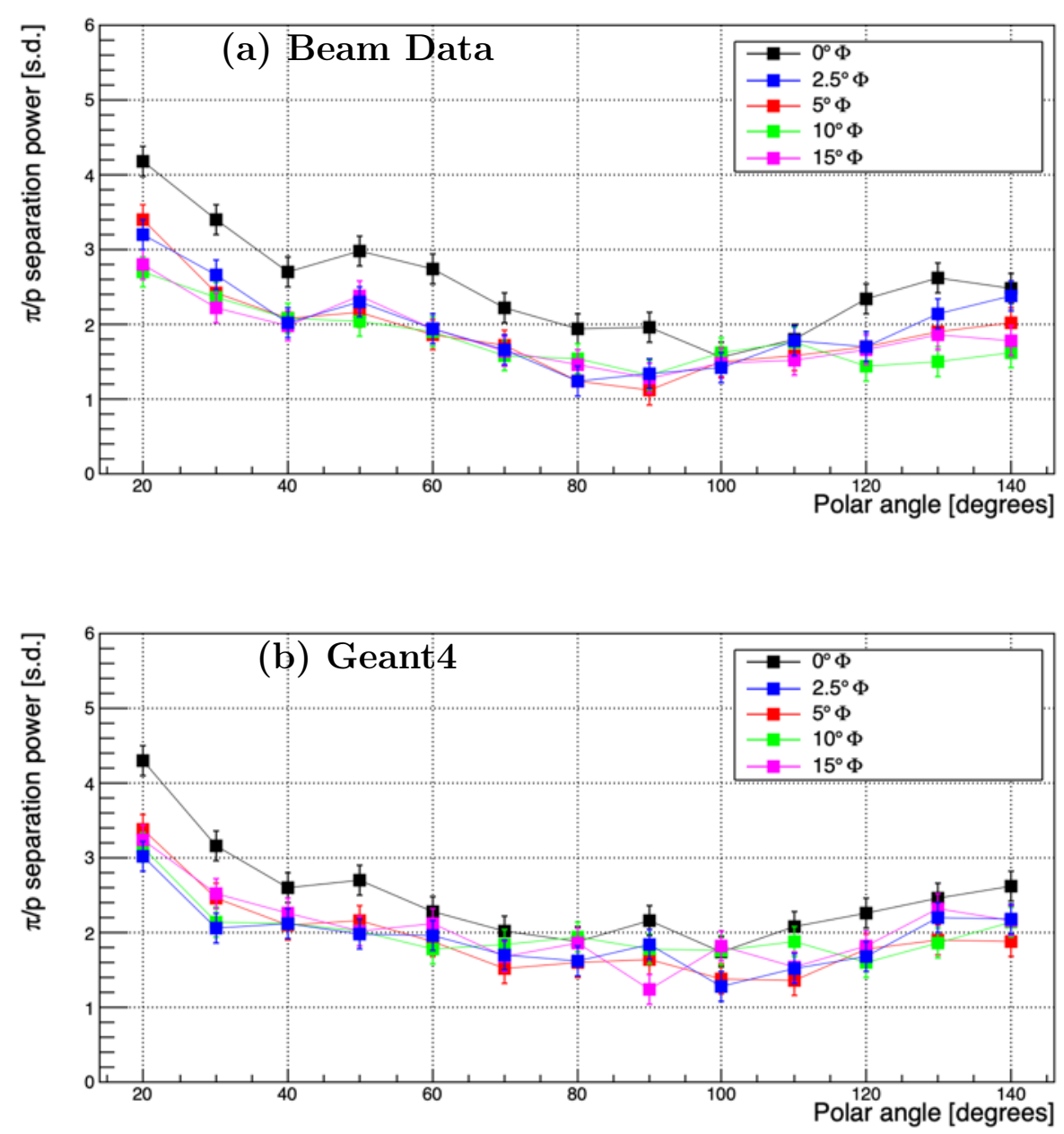

Figure 4.52: Pion/proton separation power as a function of the prototype polar angle with $7 \mathrm{GeV} / \mathrm{c}$ momentum at different values of the azimuthal angle $\Phi$ for beam data as a result of Cherenkov angle data PDF (a) and Geant4 simulation using geometrical reconstruction (b). 

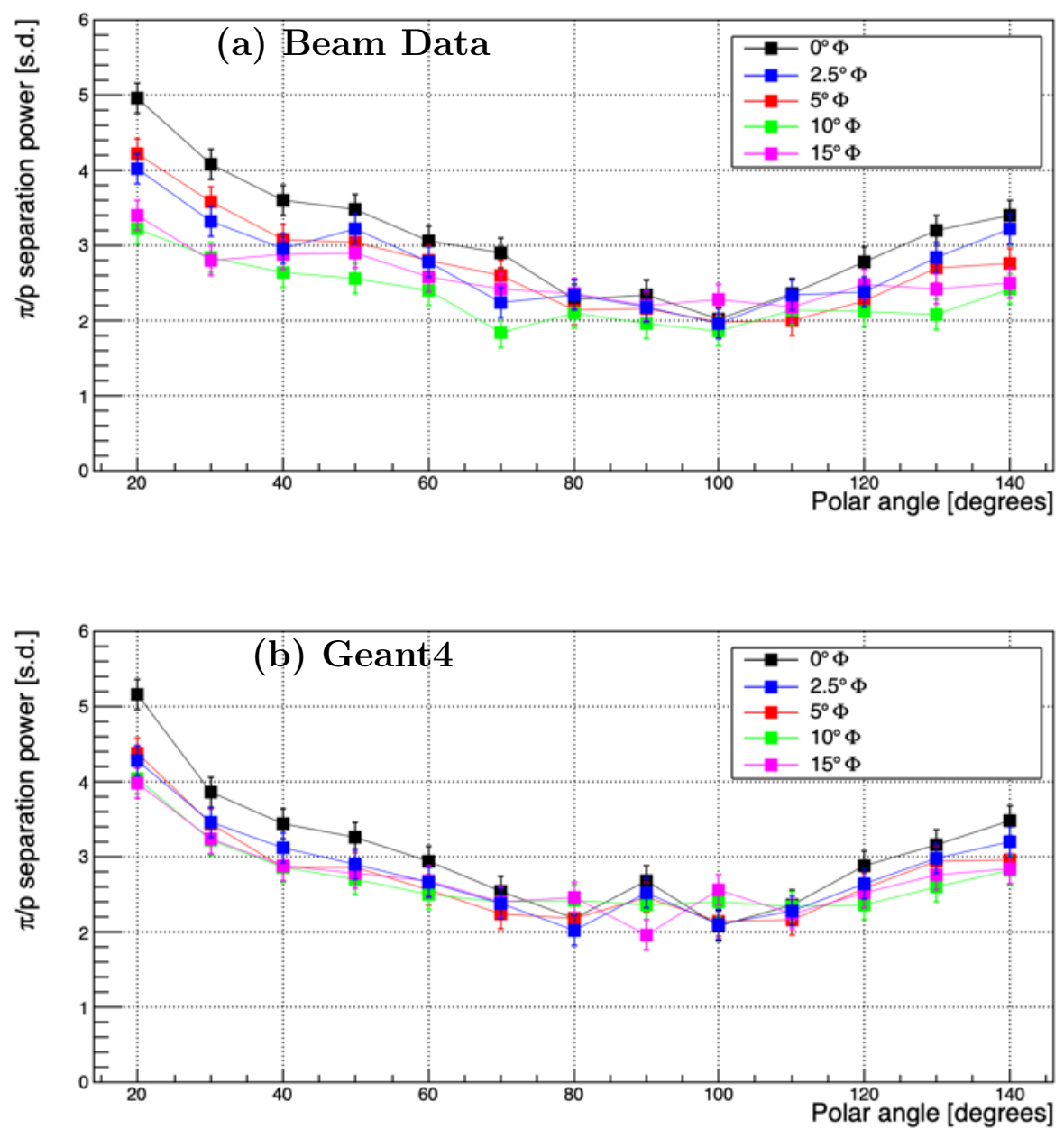

Figure 4.53: Pion/proton separation power as a function of the prototype polar angle with $7 \mathrm{GeV} / \mathrm{c}$ momentum at different values of the azimuthal angle $\Phi$ as a result of time-based imaging for beam data (a) and Geant4 simulation (b). 


\subsection{Summary}

The geometrical reconstruction is a fast algorithm, which is used to study DIRC detector figures of merits and the PID performance of the near-final design of the PANDA Barrel DIRC in a test beam environment. The standard geometrical reconstruction method is sensitive to the misalignment and calibration. It requires additional corrections to reach the expected performance. The Cherenkov data PDF is a newly developed algorithm for this thesis, based on the geometrical reconstruction. The main feature of this method is that it is less dependent on proper alignment and calibration, providing comparable performance with the standard geometric reconstruction after detailed correction procedures. However, this method is more suitable for the test beam environment where sufficient statistics can be collected to generate the Cherenkov angle data PDFs. The time imaging PID method using data PDFs has superior performance to the geometrical reconstruction. However, creating these PDFs for each angle, position, momentum, and particle type require large storage resources. These issues would be solved with time imaging utilizing analytically generated PDFs, but this has not yet been implemented for the Barrel DIRC. It remains to be seen if the PID performance is competitive with the results from the standard time-based approach. Figure 4.54 shows the pion/proton separation power for different PID methods at $20^{\circ}$ polar angle.

The 2018 beam campaign successfully validated the near-final design with $2 \times 4 \mathrm{MCP}$ PMT providing similar PID performance as the design with $3 \times 4$ MCP-PMT described in the technical design report (TDR), leading to change of the design with a substantial cost saving. The analysis results of the prototype test at CERN were shown to be in excellent agreement with simulation, validating the PANDA Barrel DIRC Geant4 simulation.

PID methods comparison

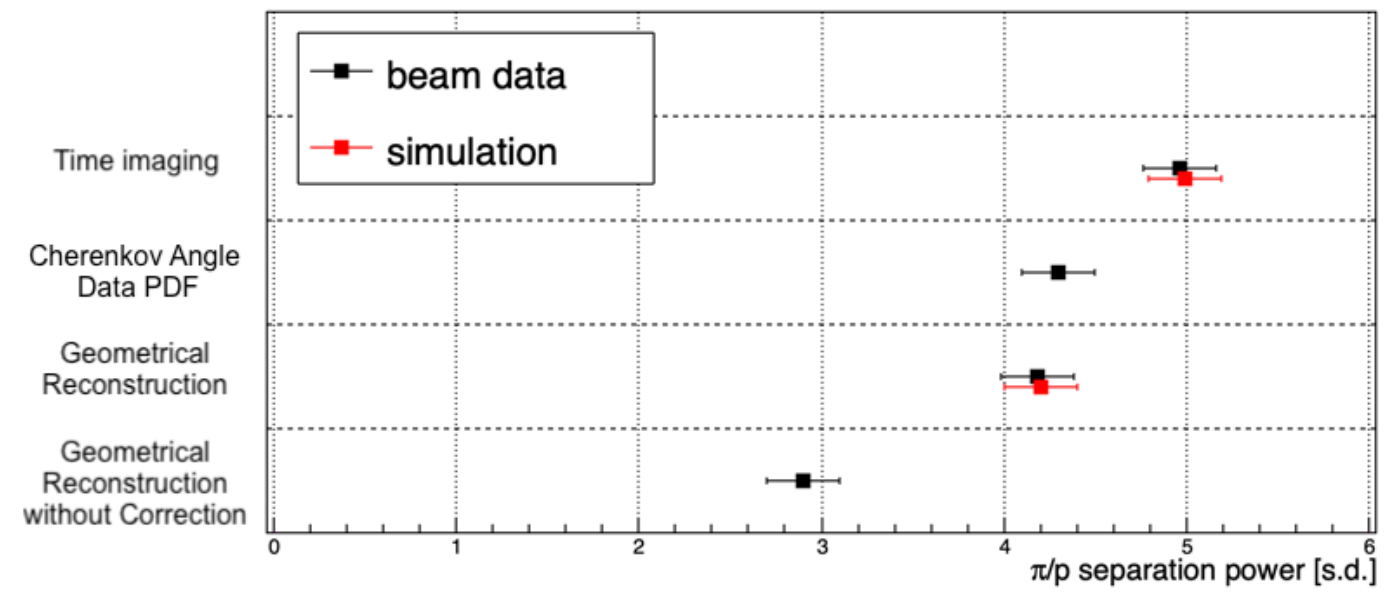

Figure 4.54: $\pi / \mathrm{p}$ separation power for data (black) and simulation (red) as result of the time-based imaging, the geometrical reconstruction and the Cherenkov angle data $\mathrm{PDF}$ at $7 \mathrm{GeV} / \mathrm{c}$ momentum and $20^{\circ}$ polar angle. 


\section{Chapter 5}

\section{The GlueX DIRC}

\subsection{Introduction}

The main feature of this chapter is to validate the PANDA Barrel DIRC simulations and reconstruction methods using collider beam data [47], as part of the FAIR Phase-0 program. GlueX [47] is a fixed target $\gamma \mathrm{p}$ experiment using a liquid hydrogen target with a polarized photon beam, located at the end of a beamline from the continuous electron beam accelerator facility at the Thomas Jefferson National Accelerator Facility (JLab) in the experimental area, Hall D. The GlueX experiment was designed to search for and ultimately study the properties of hybrids mesons (an unconventional class of mesons), which contain an intrinsic gluonic degree of freedom in their wave functions. Lattice QCD calculations predicted these hybrid meson states, providing an opportunity to quantitatively test our understanding of the strong nuclear force in this non-perturbative regime [48]. The Continuous Electron Beam Accelerator Facility (CEBAF) (shown in Fig. 5.1) at JLab provides a continuous wave $12 \mathrm{GeV}$ electron beam to Hall D. The GlueX spectrometer is a nearly $4 \pi$ hermetic detector capable of detecting neutral and charged particles and performing robust particle identification. The electron beam is used to produce a photon beam with tagged energy to allow for exclusive events reconstruction. The general description of the GlueX experiment is as follows: the photon beam is produced, tagged, collimated and steered on the target in the beamline upstream the main GlueX detector, two electromagnetic calorimeters are responsible for detecting neutral particles, two tracking gas chambers to detect charged particles and different sub-systems contribute to particle identification. The main components of the beamline are shown in Fig. 5.2 and Fig. 5.3.

Construction and installation of the baseline GlueX Phase I detector was accomplished in 2014. Since then, a substantial commissioning program has been performed in Hall D, with first photon beams delivered in the Fall 2014 and continuing through the final commissioning runs and the physics runs in Spring 2016 [51]. The particle identification (PID) capabilities have been studied with this data, and in the forward region and the time-of-flight (TOF) detector performance has reached its design specifications for providing $\pi / \mathrm{K}$ separation up to $\mathrm{p} \approx 2 \mathrm{GeV} / \mathrm{c}$.

For Phase II of the GlueX experiment, an upgrade (the DIRC) is needed to enhance the PID capabilities to study the strange quark flavor content of hybrid states, thus 


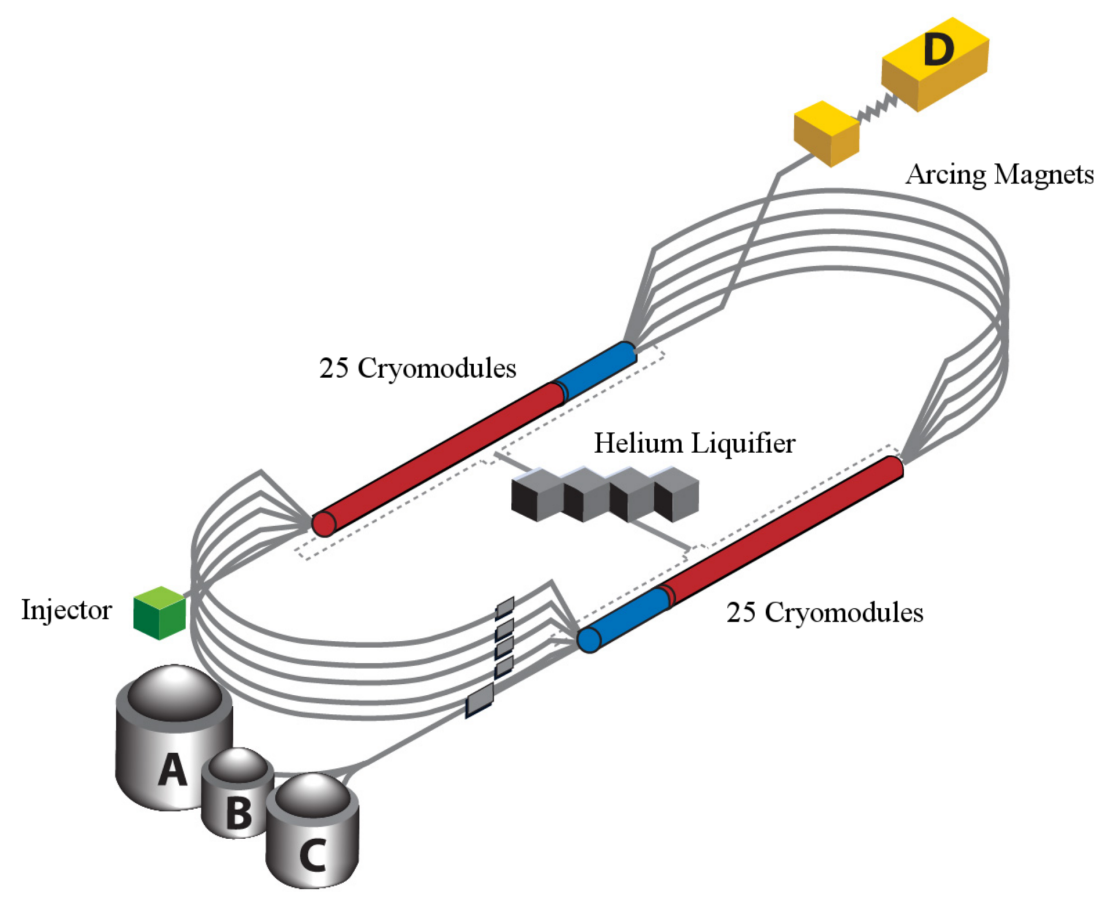

Figure 5.1: CEBAF at Jefferson Lab. A high-frequency electron beam is accelerated to high energy and supplied to up to four experimental halls simultaneously [49].

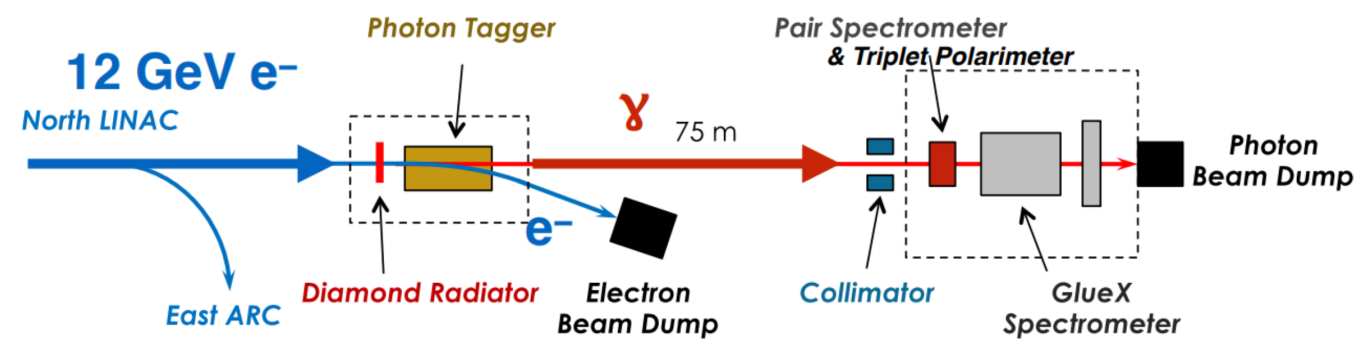

Figure 5.2: Schematic of the GlueX beamline components and the spectrometer [50]. 


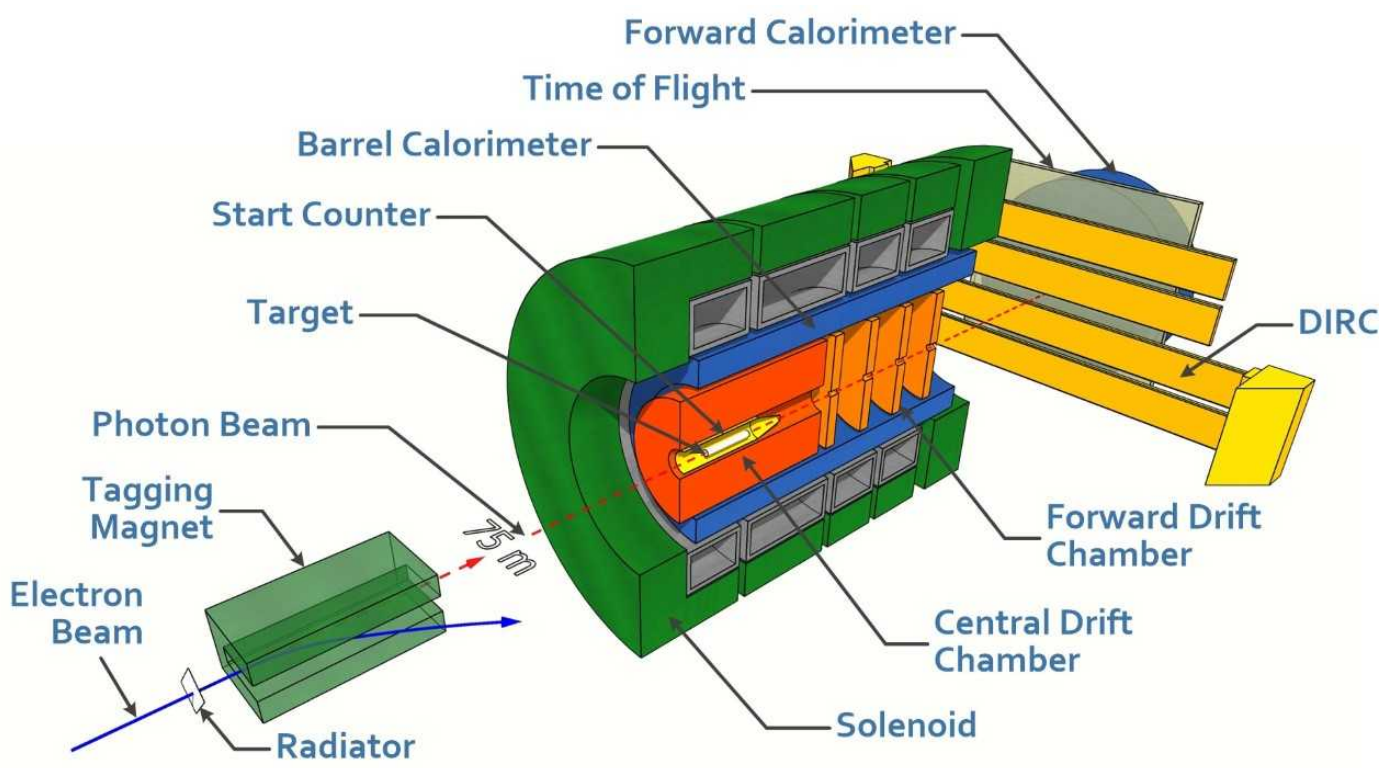

Figure 5.3: The GlueX beamline and spectrometer.

exploiting the full discovery potential [52]. The GlueX DIRC utilizes one third (four modules) of the fused silica radiators from the decommissioned BABAR DIRC detector [24], attached to two newly constructed compact expansion volumes (known as Optical Boxes), inspired by the SuperB FDIRC prototype [25], which are equipped with pixelated detector readout, see Fig. 5.4. The DIRC upgrade will expand the PID capability beyond $2 \mathrm{GeV} / \mathrm{c}$, up to $3.7 \mathrm{GeV} / \mathrm{c}$.

\subsection{Detector Construction and Installation}

The first primary mission of the GlueX DIRC installation project was to transport a four DIRC bar boxes from SLAC to JLab in 2018. These bar boxes are recycled from the decommissioned BABAR experiment consisting of twelve $4.9 \mathrm{~m}$ long fused silica radiator bars, contained in an aluminum housing [24]. Transporting the very fragile DIRC bar boxes from SLAC to JLab, after a decade in storage, was a major concern and logistical challenge. After detailed assessment, the bars were transported in two separate shipments by a truck with real-time careful monitoring. Customized crates for the transportation were designed and constructed. Each hosted mechanical and air suspension systems, accelerometers, an ultra-pure $N_{2}$ purge system, and cameras to monitor and ensure safety [52]. For a detailed discussion of the transportation system, see Ref. [55]. Once at JLab, these bar boxes were installed in their support structure for operation in Hall D, as shown in Fig. 5.5.

\subsubsection{Bar Boxes}

Four BABAR DIRC bar boxes, number 0, 1, 10, 11 [24] (out of twelve bar boxes), were selected to construct the GlueX DIRC. They were transported from SLAC, where all the bar boxes had been stored after the BABAR experiment was disas- 
(a)

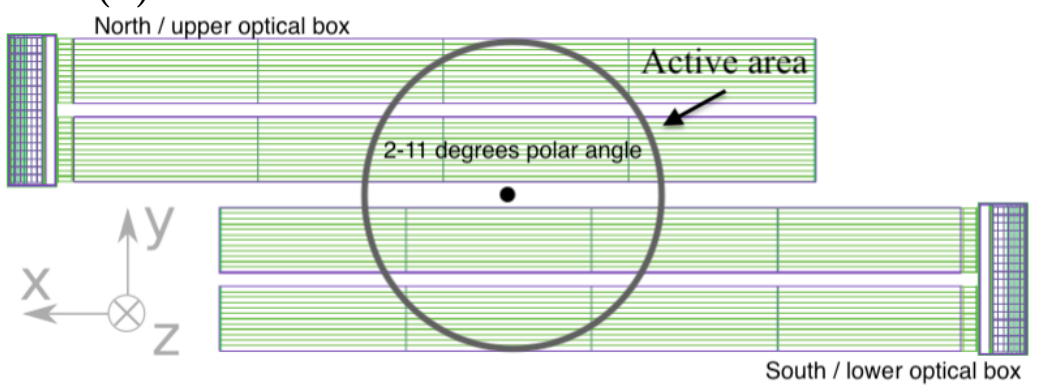

(b)

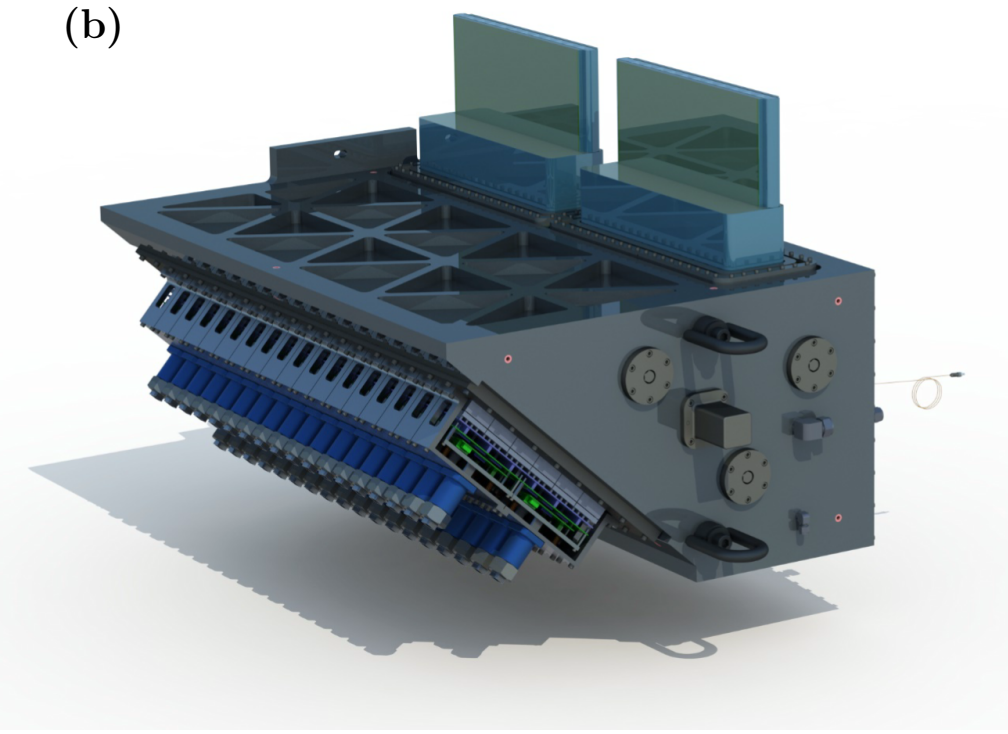

(C)

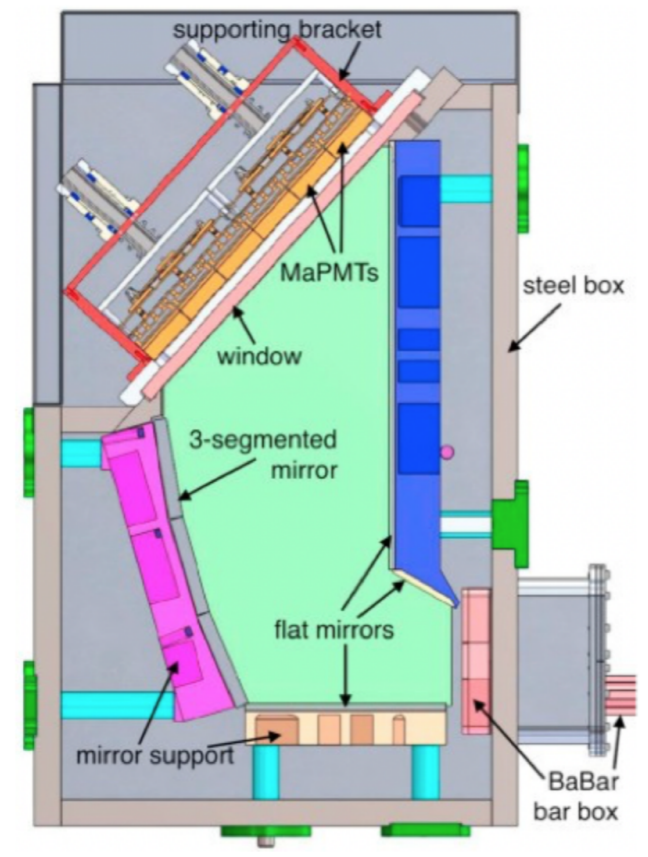

Figure 5.4: Schematic drawing of the whole GlueX DIRC (a), 3D drawing of the optical box [53] (b), layout of the DIRC optical box showing the locations of optical components, including: PMTs, mirrors, and fused silica bar box attachment points [54](c). 


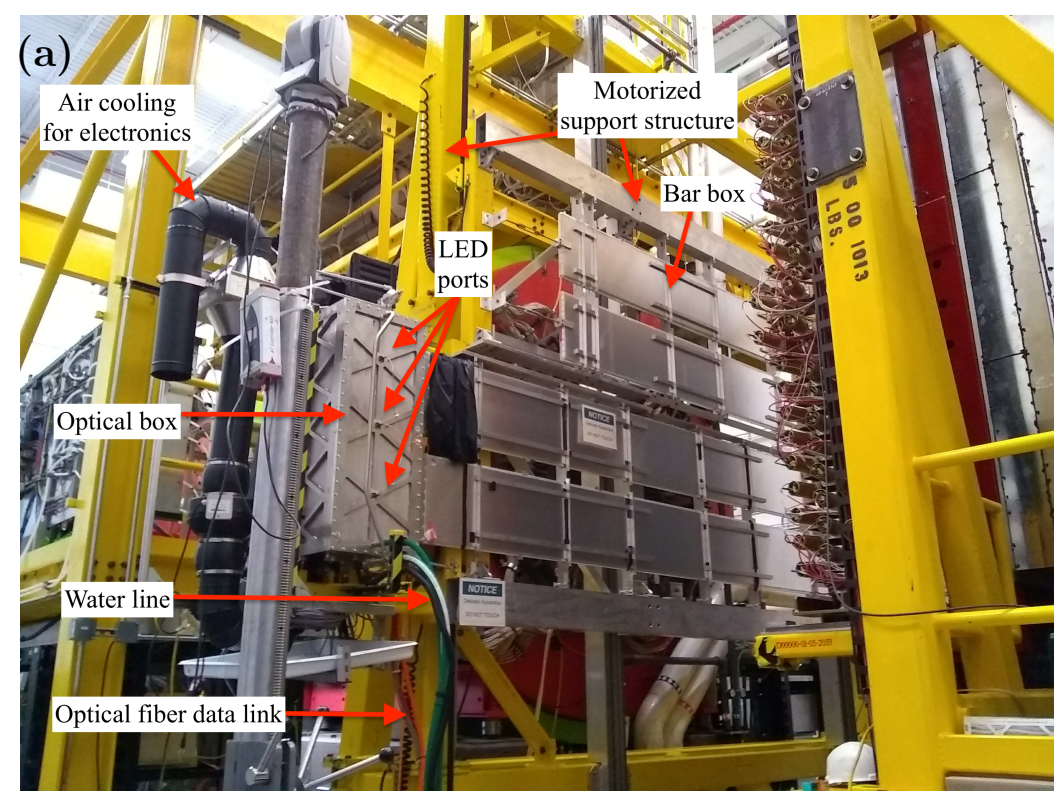

(b)

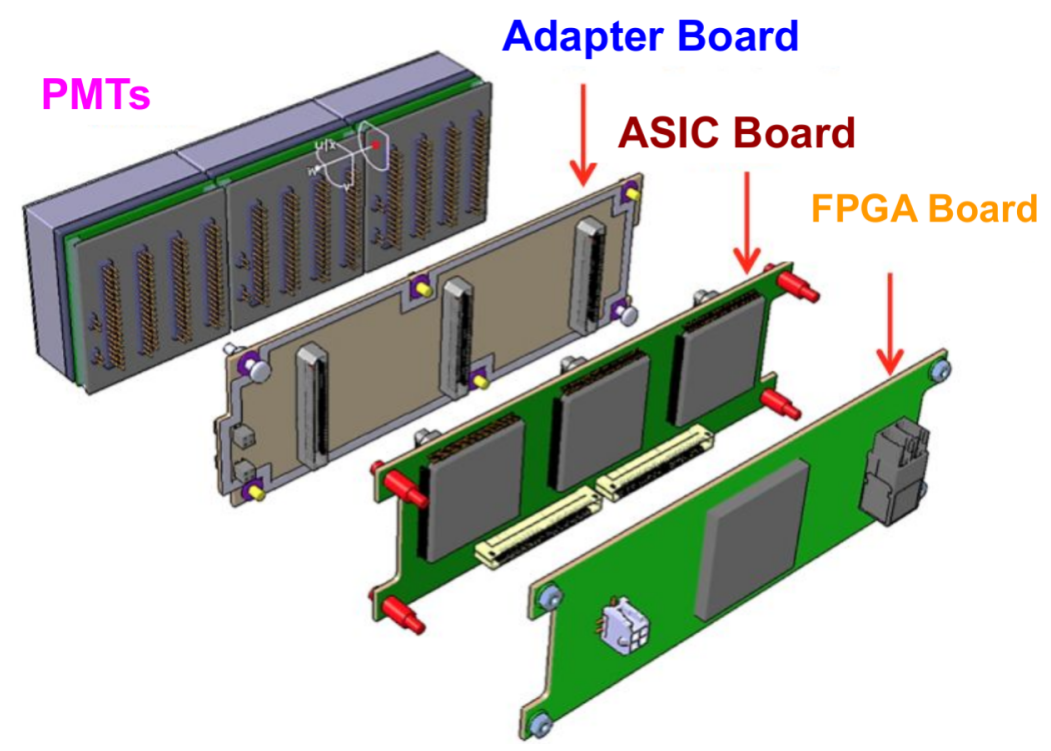

Figure 5.5: Four BABAR bar boxes after transport to JLab installed in the GlueX detector on the DIRC support structure in Hall D [54](a). Layout of the DIRC PMT, the electronics design is based on the CLAS12 RICH with about 1 ns timing resolution [56] (b).

sembled in 2010. The integrity of the glue joints and bar surfaces during transport was maintained by the design of the complex transport crate and detailed monitoring system, see Fig. 5.6a. The schematic of a bar box is shown in Fig. 5.6b. It is a single unit with 12 long quartz radiator bars $\left(34 \times 17.25 \times 4900 \mathrm{~mm}^{3}\right)$ inside.

\subsubsection{Expansion Volume}

The initial expansion volume of the BABAR DIRC detector was large and filled with 6000 liters of purified water [24]. The design was updated for the SuperB 
(a)

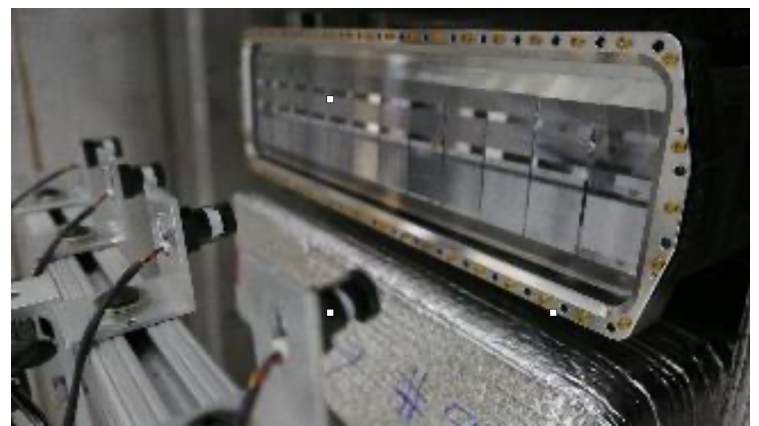

(b)

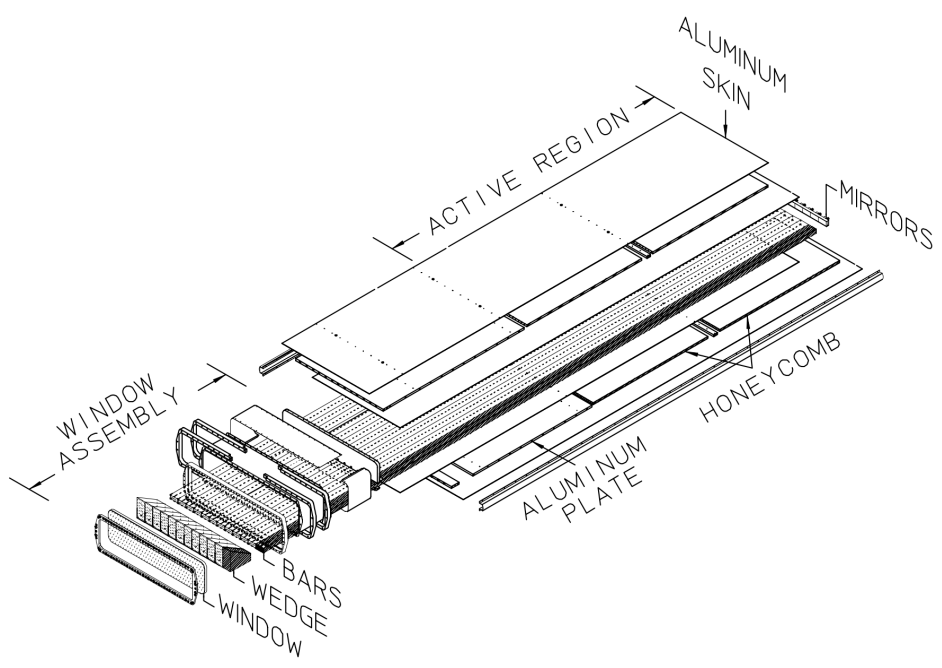

Figure 5.6: The monitoring cameras insure the quality of the bars and the glue joints during the transportation (a). Schematic of the BABAR DIRC bar box [57](b).

experiment to include focusing mirrors that enable a much more compact design [25], therefore, minimizing backgrounds and reducing costs by limiting the area of the photo-detector plane. This focusing DIRC (FDIRC) was designed and prototyped at SLAC [25] with the constraint that the BABAR bar boxes could not be altered. The GlueX DIRC optical box design began with the FDIRC prototype design. For BABAR a small wedge is glued to the readout end of each bar and a flat mirror was attached to the other end. A requirement for the reuse of the bar boxes was that they remain unchanged, which means that the small wedges have to be left in the optical system of the GlueX DIRC without modifications.

The SuperB FDIRC design utilized a cylindrical mirror to focus the Cherenkov light onto the PMT plane. For GlueX this was replaced with a three-segment approximation of the cylindrical curvature, using only flat mirrors to simplify the design and reduce the cost, as seen if Fig. 5.4. The optimized radius of the mirror approximated by three flat segments allows using fewer photo-sensors, the main cost driver for the GlueX DIRC. The resolution of the Cherenkov angle is approximately the same for the designs with the cylindrical and the three-segmented mirrors [58]. Figure 5.4 shows a schematic for the GlueX DIRC optical box. The optical box contains flat mirrors on all sides together with the multiple flat mirrors are used to direct the light 
to a plane of Multi-Anode PMTs (MAPMT) that interface with the water volume via a fused silica window. The alignment of the bar boxes in GlueX DIRC permits using one common expansion volume for two bar boxes. The photo-sensors are optically coupled to the photon camera using silicone cookies [26]. The material used to produce the cookies is RTV615 by Momentive [30]. The thickness of the cookies is about $0.17 \mathrm{~cm}$ with area of $5.2 \mathrm{~cm}$ times $15.8 \mathrm{~cm}$. The silicone cookies ensure good optical coupling, to reduce the photon loss in the case of air gap due to the refraction on the quartz-air interface. More details about the cookies specification are described in Ref. [59]. The photon detection efficiency of the DIRC system drops significantly at a wavelength below $300 \mathrm{~nm}$. The drops are primarily due to the borosilicate face of PMTs, Epotek 301-2 glue (for the fused silica bars, wedges, and windows), and water absorption. The water in each of the optical box $(\approx 450$ liters) is circulated continuously at a rate of 10 times per day, to ensure the quality of the photon transmission [52].

\subsubsection{Photon Detection and Readout}

The imaging of Cherenkov photons for the GlueX DIRC detector requires a photodetector with good position resolution and moderate timing resolution that can operate (with appropriate shielding) in the fringe magnetic field from the GlueX solenoid. Given these requirements, the Hamamatsu H12700 [60] MAPMTs are used as the photon detector for the GlueX DIRC. The active area of a single MAPMT is 48.5 $\mathrm{mm} \times 48.5 \mathrm{~mm}$ and is segmented in 64 individual pixels [27]. One advantage of this choice is a synergy with the RICH detector for CLAS12 [61] at Jefferson Lab, which also uses the H12700 and has similar requirements for the readout system. Therefore, the GlueX DIRC utilizes the same electronics as the CLAS12 RICH, based on the MAROC3 chip [62], which was specially designed for the readout of 64-channel MAPMTs. The MAPMTs were subjected to a dedicated quality assurance test. A laser beam is used to illuminate the effective detection area to measure the single photo-electron response and the transit time-spread for each pixel individually to determine the gain and quantum efficiency, following the procedure described in Ref. [63]. Each optical box is equipped with 36 PMT module assemblies. The PMT module assembly consists of three PMTs, a high voltage adaptor board, an application-specific integrated circuited (ASIC) board, and an field-programmable gate array (FPGA) board as seen in Fig. 5.5. For each optical box there are 90 PMTs and 18 plastic square dummy PMTs are mounted. The dummy PMTs are used as a mechanical support, then the total number the DIRC readout channels for both optical boxes is 11520 .

\subsubsection{Calibration System}

The calibration system is equipped with an LED pulser with (405 nm wavelength, $150 \mathrm{~Hz}$ operates with single photon mode to provide $<10 \%$ occupancy per pixel) and reference Silicon Photomultiplier (SiPM). For each optical box the light is transported using three LED optical fibers through the feed-through ports. Each LED source is diffused into a square pattern by an engineered diffuser with an opening 
angle of 50 degrees (in the air) to cover one-third of the detection plane. The LED sources are configured to be 10 ns apart. The timing from the reference SiPM together with the detected photon time per pixel, are used to remove the time offsets between pixels, due to cable length and channel-to-channel differences inside the photon sensors. The removal of this time offsets are mandatory to achieve the required time resolution for the GlueX DIRC. Pixel-by-pixel $T_{0}$ values are provided by the calibration system and stored in a database to calibrate the photon arrival times of all channels.

The global timing calibration for the DIRC detector is performed same way as the other GlueX sub-detector, by applying overall time shift of all photo-sensors with respect to the relevant physics trigger. These valuable data are not only useful for the live calibration; they also offer essential insight into the long term performance of the detector system, including water quality, detector efficiency, and data acquisition $[52,59]$. Figure 5.7 a shows the time-walk of the single photo-electron signal as a function of the Time-over-Threshold (TOT), the time distribution for the three calibration fibers (with a 10 ns delay between fibers) of the south optical box is illustrated in Fig. 5.7b and figure 5.7c shows the time resolution per channel, before and after applying the time walk correction.

\subsection{The GlueX DIRC Simulation and Reconstruc- tion}

Verification of the PANDA Barrel DIRC simulations and reconstruction methods using GlueX DIRC beam data presents an ideal testing environment, to apply lessons learned to PANDA, as part of the FAIR Phase-0 program. The GlueX Geant4-based software has a detailed description of the DIRC detector geometrical specifications and optical system, including the definition of the materials optical properties, the realistic structure of the photo-sensors, implementation of the quantum efficiency of the MAPMT, and the reflection probability for the BABAR radiators based on the SLAC measurements [24]. The DIRC support structure within the geometrical acceptance was implemented as well.

\subsubsection{Reconstruction and PID methods}

\section{Geometrical Reconstruction}

The geometrical reconstruction method, based on the PANDA barrel DIRC algorithm, was deployed in the GlueX software and has been used during the detector commissioning and the nominal DIRC operation. The PID using the geometrical reconstruction is based on the look-up table approach. A look-up table was created for every bar in advance before the analysis stage by simulating a large number of photons generated from a single point at the readout end of the radiator bar. The LUT includes all photon reflections from the three-segment mirror, the wedge, and the side mirrors. The look-up table contains information about the pixel number 
Photon time
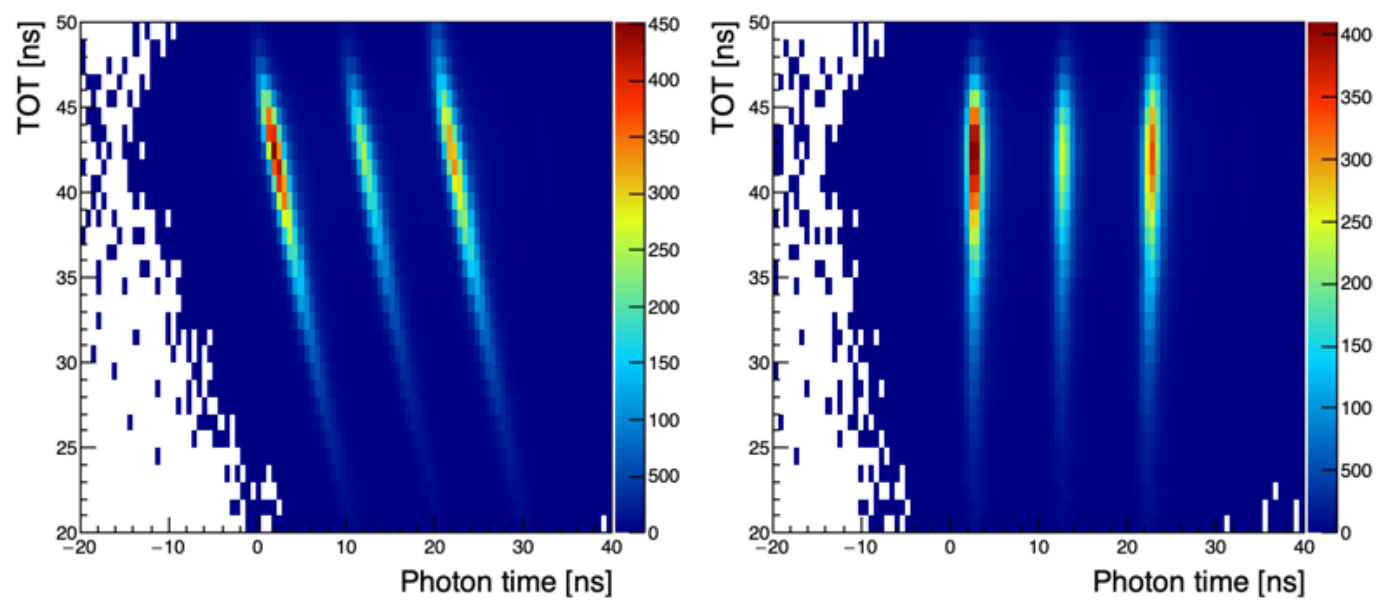

Time of single photo-electron
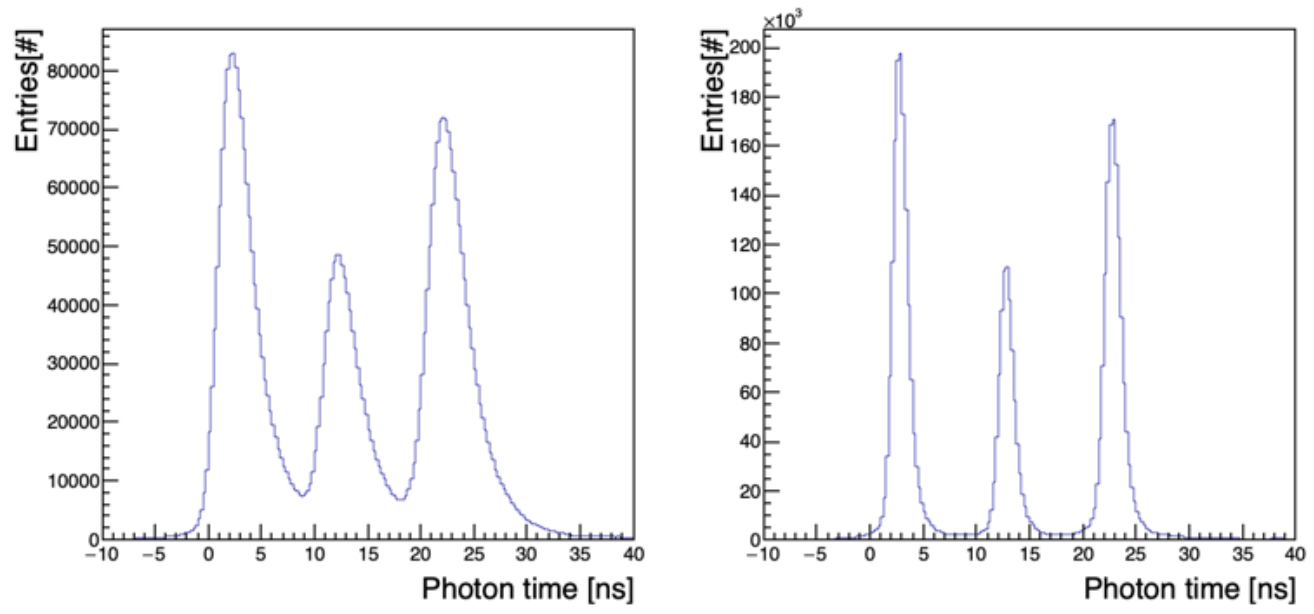

Time resolution
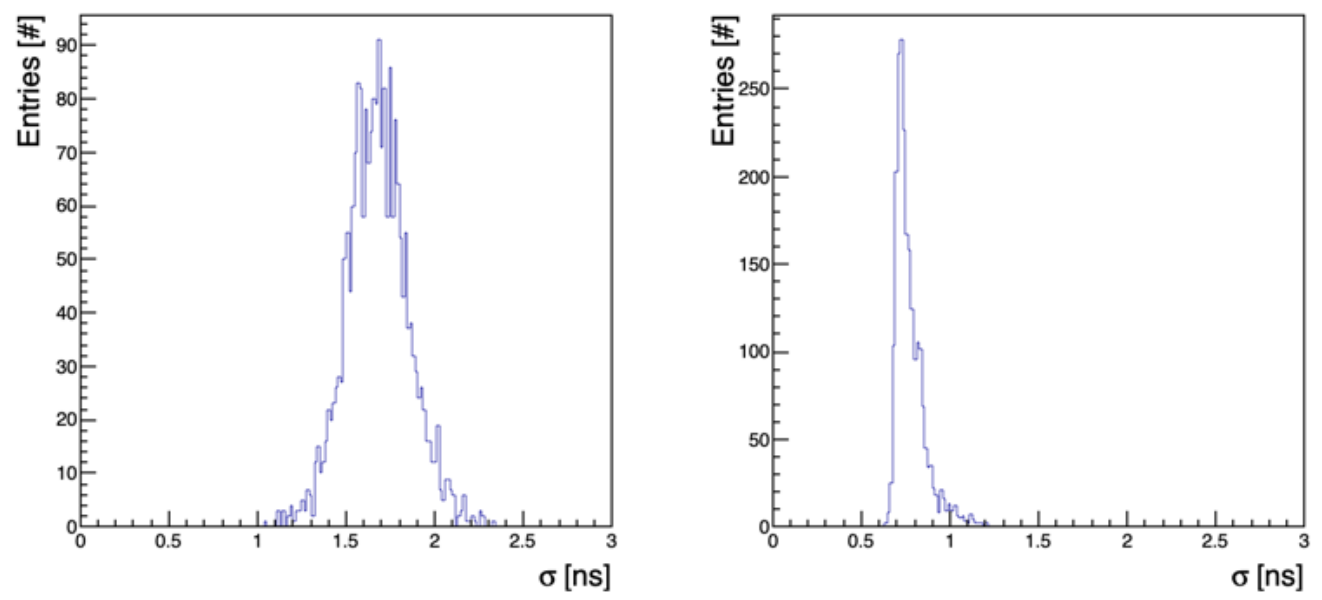

Figure 5.7: Photon time (top), time of single photo-electron signal (middle), time resolution (bottom) before (left) and after (right) applying the time walk correction for randomly selected pixels from the south optical box. 
and photon directions at the exit from the bar, together with the associated propagation time for each direction. The reconstructed Cherenkov angle is then defined using the LUT information together with the charged track direction provided by the tracking system.

\section{Time-imaging}

The basic concept of the time-imaging is that the measured arrival time of Cherenkov photons in every single event and every pixel is compared to the expected arrival time for every particle hypothesis, yielding the PID likelihoods. A detailed description of this method is described in section 3.4.2. Given that the limited calibration sample size adds a major challenge to use this method, even though an attempt to create time imaging data PDFs for large $\mathrm{x}$ bins was not successful and kept for possible future work once enough statistics to be collected. Besides the analytical method is still in preparation.

\section{FastDIRC method}

The FastDIRC method is a semi-analytic approach to transporting Cherenkov photons through the DIRC to generate a probability density function for each particle hypothesis kernel density estimation (KDE). A detailed description of the method can be found in Ref. [64, 65].

\subsection{Preliminary Commissioning Results}

Two DIRC commissioning periods took place in 2019: the first was latest for 10 days in February 2019, when only the lower half of the GlueX DIRC was commissioned at the nominal operating condition of Phase I of the GlueX experiment. For the second period the data was collected for two weeks in December 2019, where the full detector was assembled and commissioned, and the operation at GlueX Phase II (high intensity) condition was also tested [52]. The results on this chapter are based on the data collected within December 2019. Sections 5.4.3 and 5.4.4 show the initial results of the reconstructed Cherenkov angle and the photon yield. Section 5.4.5 show the PID performance and a path to improvements with alignment.

\subsubsection{PID Calibration Physics Channels}

To quantify the GlueX DIRC PID performance, a kinematically identified sample of kaons and pions from external PID systems are needed. The nominal GlueX experiment provided high statistics samples of exclusive $\gamma \mathrm{p} \rightarrow \rho \mathrm{p}, \rho \rightarrow \pi^{+} \pi^{-}$and $\gamma \mathrm{p} \rightarrow \phi \mathrm{p}, \phi \rightarrow K^{+} K^{-}$events see Fig. 5.8.

The selection of the events was performed by applying a cuts on the invariant mass window between $\left(600-900 \mathrm{MeV} / \mathrm{c}^{2}\right)$ to select the $\rho$ events and a window between (1010-1026 MeV/ $\mathrm{c}^{2}$ ) for $\phi$. The selection criteria are also requiring that the missing mass squared $<0.01 \mathrm{GeV} / \mathrm{c}^{2}$ and the kinametic fit $\chi^{2} / N D F<2.0$ for $\phi$ events and 
$\chi^{2} / N D F<10.0$ for $\rho$ events.
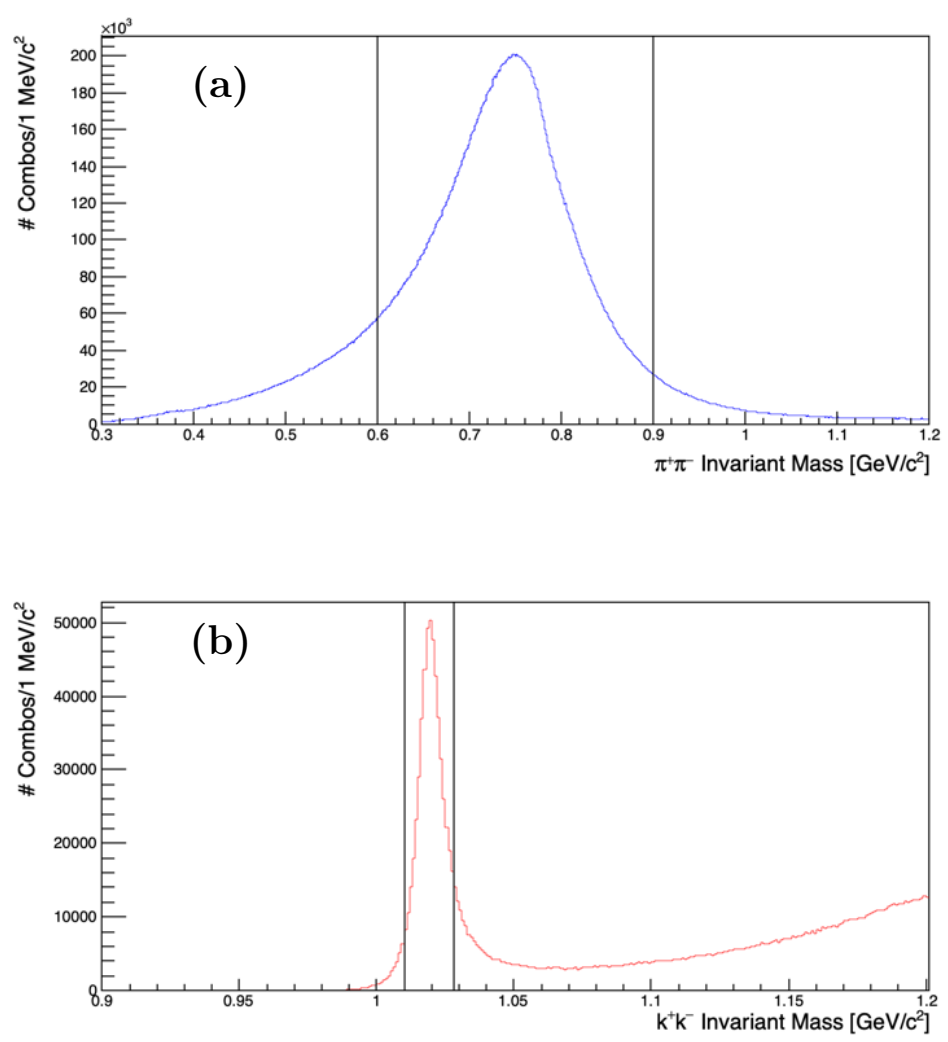

Figure 5.8: Invariant mass of $\pi^{+} \pi^{-}$(a) and $K^{+} K^{-}$(b) showing clear $\rho$ and $\phi$ peaks selected to study the DIRC detector performance.

These event samples provide the kinematically identified samples for $\pi^{ \pm}$and $\mathrm{K}^{ \pm}$ tracks, which are used to test the DIRC reconstruction methods and the overall particle identification performance. The occupancy distribution of pions and kaons on the DIRC wall is illustrated in Figure 5.9. The data sample belongs to the $\rho$ and $\phi$ reactions. The hit position of each charged particle on the active area of the DIRC wall (see Fig. 5.4a) is extrapolated from the tracking system upstream of the DIRC. The occupancy map shows symmetric distribution on both the $\mathrm{X}$ axis and Y-axis. The central empty area around $y=0 \mathrm{~cm}$ is due to the space between the upper half and the lower half of the DIRC, while the other symmetric empty areas around at $y \approx \pm 55 \mathrm{~cm}$ are due to the space between the bar boxes. The selected areas defined by the black rectangles has relatively high photon yield and less reconstruction ambiguities, compared to the regions closer in radius to the beamline, for this reasons they are selected for further analyses in the following sections.

\subsubsection{Hit Pattern}

The accumulated hit pattern of the beam data for the rectangular selected regions of Fig. 5.9 is shown on the top panel of Fig. 5.10, while the bottom panel shows the analogous distribution from a simulation sample. A very good match between 


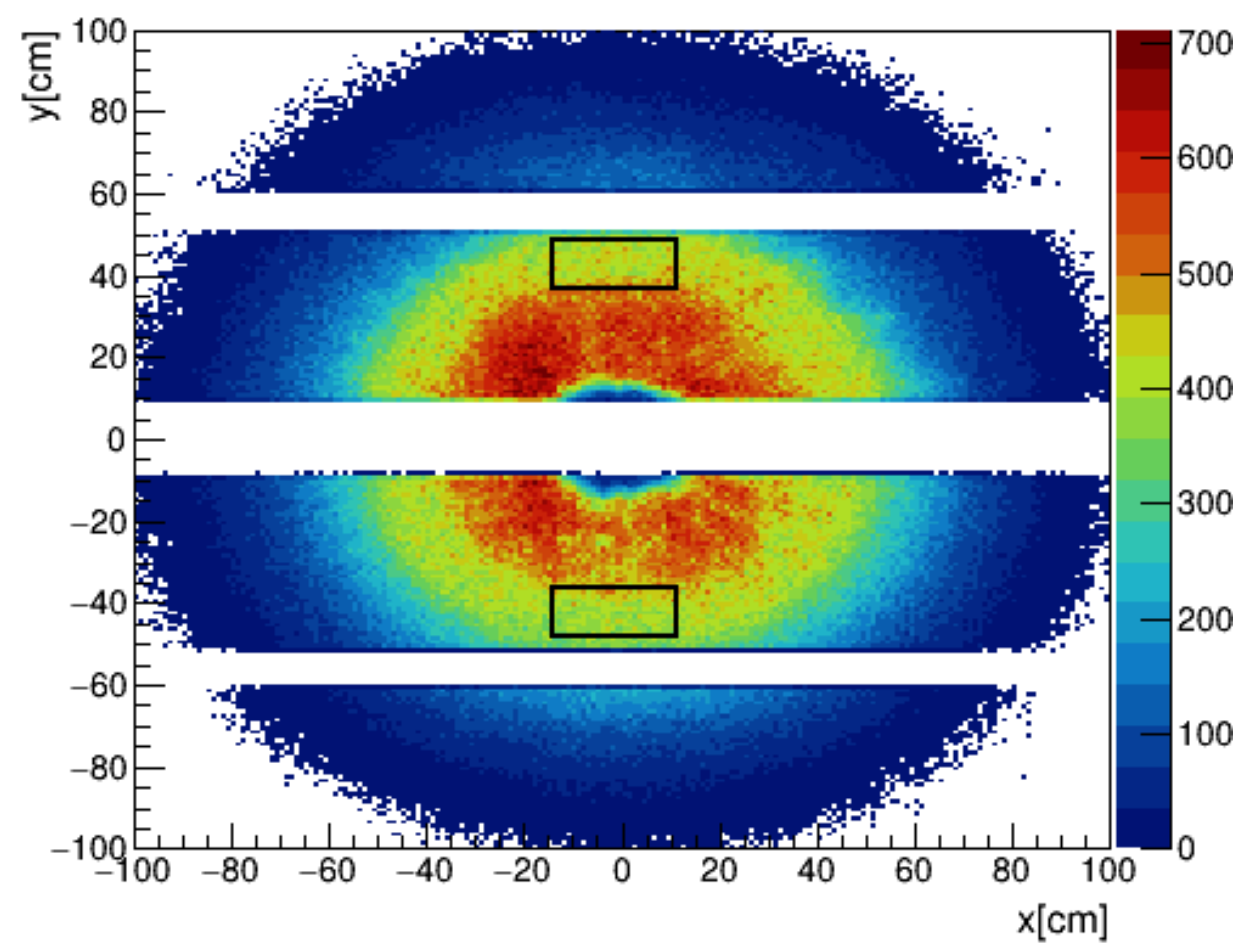

Figure 5.9: Number of reconstructed tracks on the DIRC wall from $\rho$ and $\phi$ reactions. Tracks inside the black rectangles are selected for further analyses.

the occupancy distributions for the data and simulation is obtained. The agreement between beam data and simulation indicates the validity of the DIRC geometry implementation in the GlueX simulation platform.

\subsubsection{Cherenkov Angle Reconstruction}

\section{Hit Selection}

The time difference distribution between the measured and the expected arrival time of the Cherenkov photons time is shown in Fig. 5.11a. The width of the bar end mirror-reflected photon (indirect photons) peak is larger than the width of the direct photon peak, resulting from the chromatic dispersion. The background around the direct photon plot arises form the reconstruction ambiguities. A 2-dimensional time difference cut is applied to suppress the reconstruction ambiguities (see Fig. 5.11b). The reason behind using a $2 \mathrm{D}$ time cut rather than $1 \mathrm{D}$ cut is to compensate the chromatic dispersion. The remains photons after the cut are used to reconstruct the Cherenkov angle and to perform the PID.

\section{Cherenkov Angle Distribution}

The distribution of the reconstructed Cherenkov angle per photon for pions and kaons for beam data is shown in Fig. 5.12a. The spectrum is obtained by selecting the tracks located in the black boxes shown in Fig. 5.9, and momentum range be- 

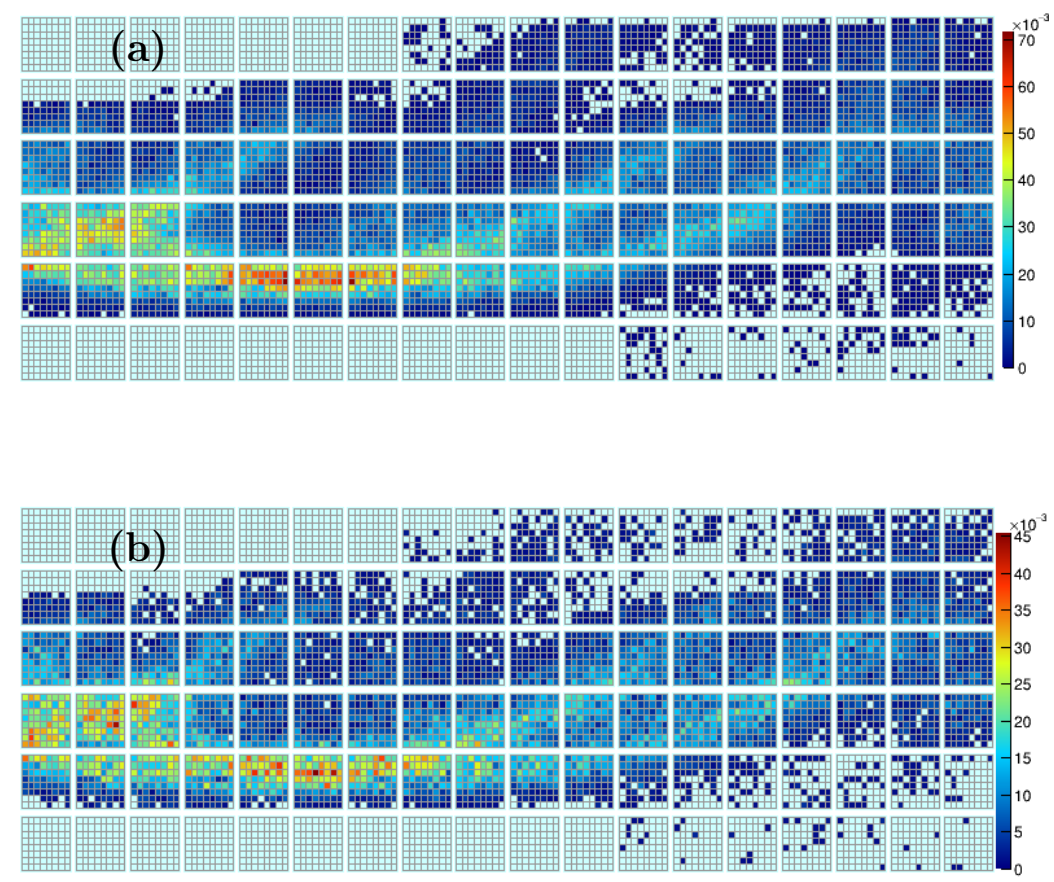

Figure 5.10: Example of the accumulated pions hit pattern for the rectangular regions of Fig. 5.9 for beam commissioning data (a), Geant4 simulation (b).

tween $[3,3.5] \mathrm{GeV} / \mathrm{c}$, identified through the $\rho$ and $\phi$ reactions described above. The distribution includes multiple reconstruction ambiguities for each detected photon, which account for the photon paths inside of the bar and the optical box (from the look-up table), which explains the background under and near the peak. The mean of the distribution is determined by fitting the peak with a Gaussian plus second order polynomial function. The mean of the fit is found to shifted with $\approx 0.002 \mathrm{mrad}$ from the expected values of the Cherenkov angles for kaons and pions at the sample average momentum with $n=1.473$ refractive index indicated by the red and blue vertical lines respectively. This shift was corrected for both pions and kaons in Fig. 5.12b. The single photon Cherenkov angle resolution (SPR) is found to be $8.4 \pm 0.1 \mathrm{mrad}$ for pions and $8.7 \pm 0.1 \mathrm{mrad}$ for kaons. A Cherenkov angle shift

\section{Single Photon Resolution}

The width of the Cherenkov angle distribution can be used to draw a map for the single photon Cherenkov angle resolution (SPR) $\sigma_{c}$, which is a characteristic of the detector performance. Figure 5.13 shows the SPR map on the DIRC wall for pion tracks identified through the $\rho$ reaction using beam data at $[3,3.5] \mathrm{GeV} / \mathrm{c}$ momentum using beam data. The reconstruction ambiguities have complicated dependencies, since the ambiguities under the Cherenkov angle distribution are different for different bar and x-axis bin combination. The main features of the SPR map are summarised as follows:

- The asymmetric nature of the optical boxes configurations contribute to the 

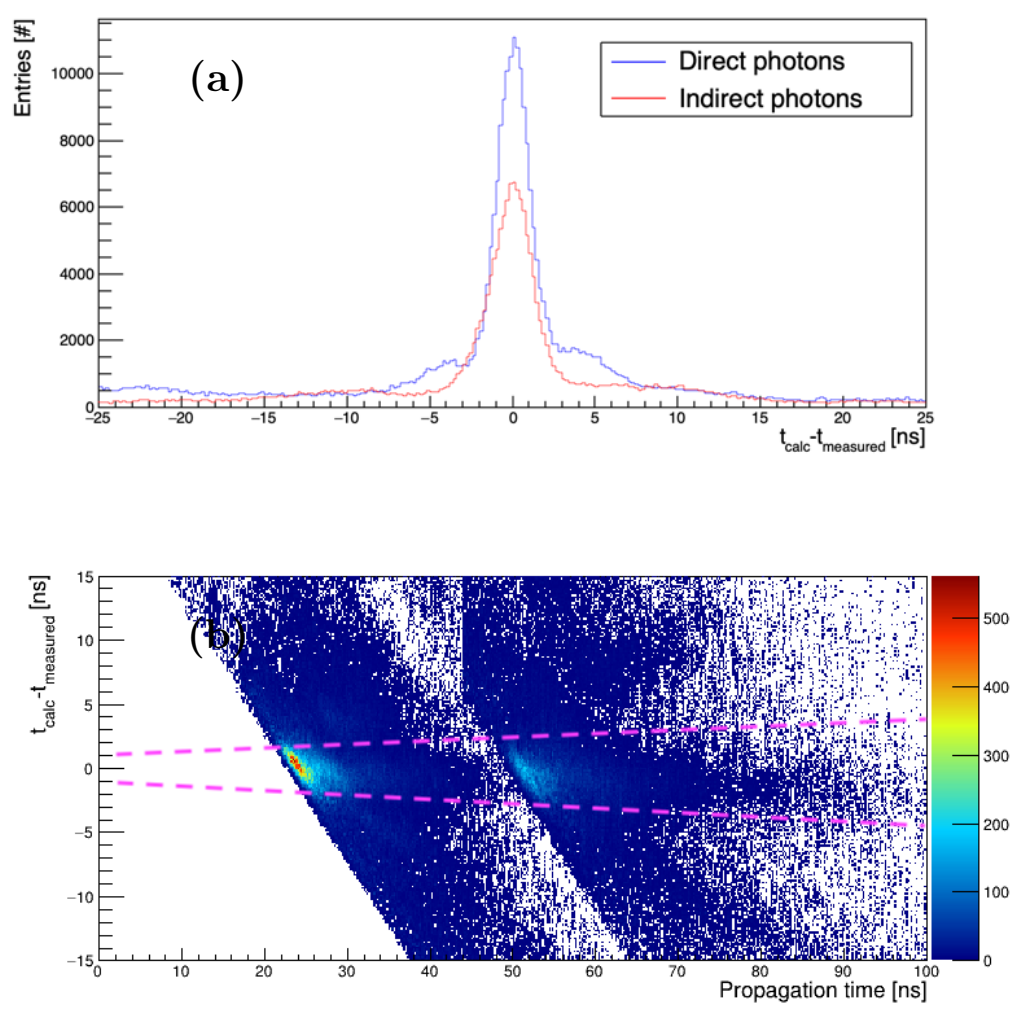

Figure 5.11: Time difference distribution between the measured and expected arrival time of the Cherenkov photons for direct photons (forward photon that did not reflect on the bar-end mirror) (blue) indirect photons (reflected on the bar-end mirror) (red) (a), 2D time difference cut (b).

asymmetric distribution of the SPR over the lower and upper halfs

- For one single bar the SPR is sensitive to the photon pathlength. The larger the contribution form long traveled photons (mirror-reflected photons or direct photons far from the optical box), the larger SPR value. This is the cause for the asymmetry of the SPR along the bar

- The modest tracking resolution together with huge background level around the beamline have an impact on increasing the SPR (see Fig. 5.14)

Figure 5.15 shows the examples of the single photon Cherenkov angle distribution after applying the 2D time cut for two kinematically identified beam data events taken at $3 \mathrm{GeV} / \mathrm{c}$ momentum inside the matched region of Fig. 5.9. The red and blue lines indicate the Gaussian plus linear function fit to the single track Cherenkov angle distribution for kaon and pion respectively. The mean values of the fitting function are in agreement with the kaon and pion mass hypothesis respectively, providing larger likelihood value for kaons comparing to pions and vice versa. 
(a) Beam data without correction

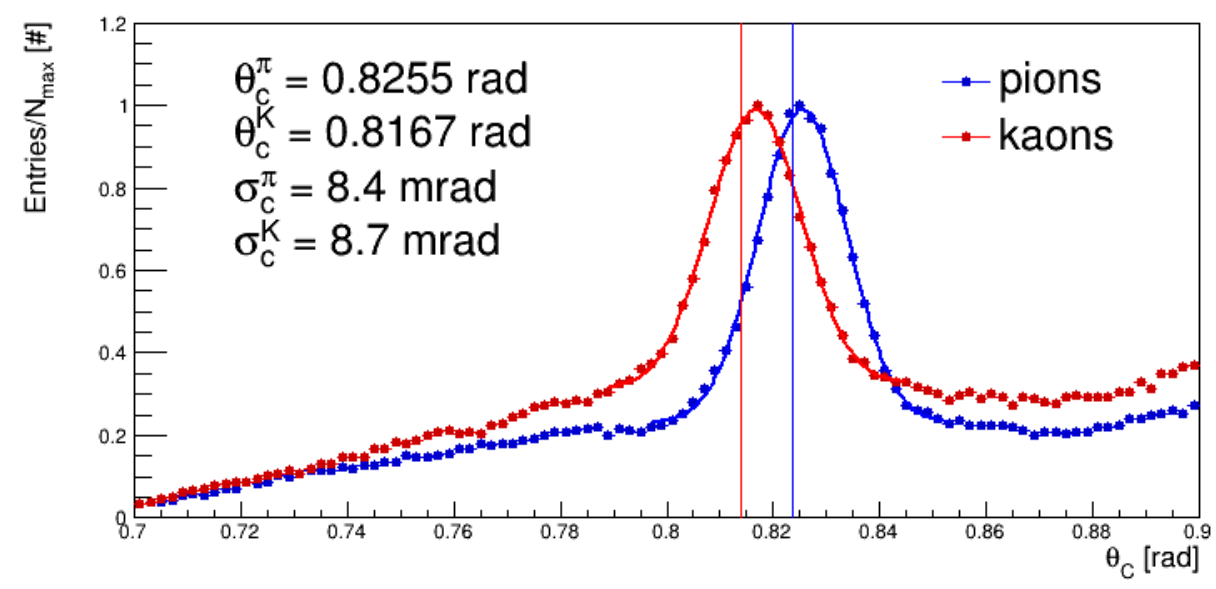

(b) Beam data after Cherenkov angle correction

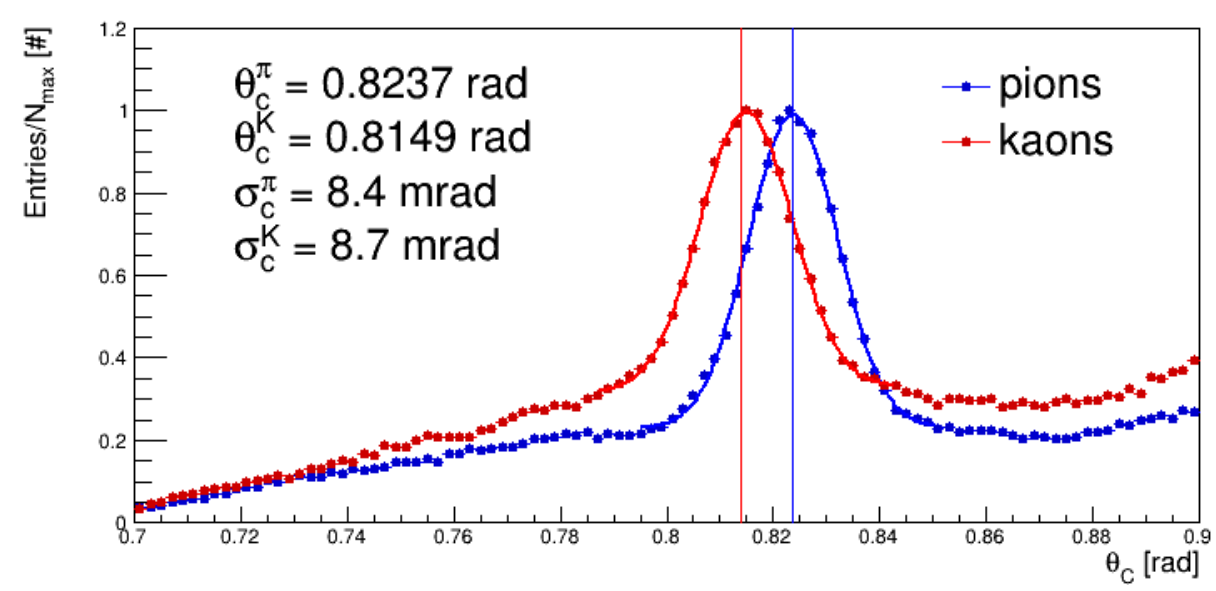

Figure 5.12: Cherenkov angle $\theta_{C}$ distribution per photon for kinematically identified pions (blue) and kaons (red) at [3,3.5] GeV/c momentum using commissioning beam data. Before applying Cherenkov angle correction (a), and after the Cherenkov angle correction (b). The vertical red and blue lines define the expected values of the Cherenkov angles for kaons and pions respectively. 


\section{Beam data}

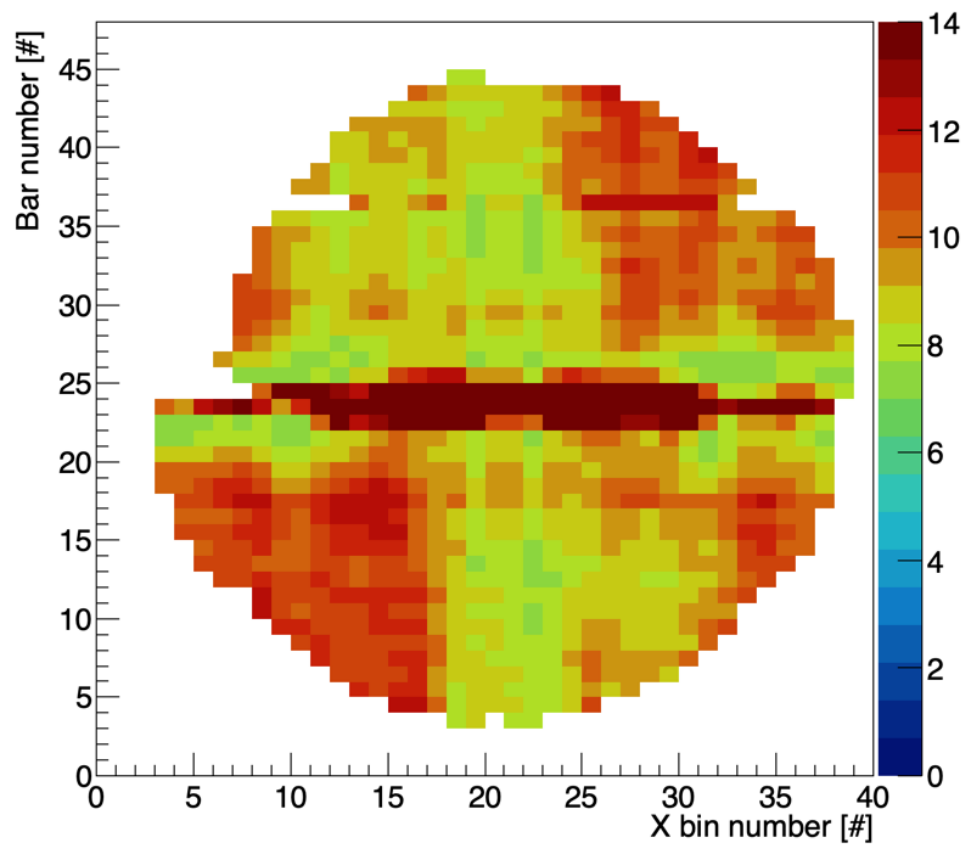

Figure 5.13: Single photon resolution map for pion tracks at $[3,3.5] \mathrm{GeV} / \mathrm{c}$ momentum, identified through the $\rho$ reaction using beam data.

\subsubsection{Photon Yield}

Photon yield is an important figure of merit which is used to study the DIRC detector performance. The determination of the number of detected photons per particle is obtained by counting the survival photons after applying the Cherenkov photons 2D cut described above and a Cherenkov angle cut around the expected value within $\pm 0.05 \mathrm{rad}$.

Figures 5.16a shows examples of the photon yield per particle for kinematically identified pions and kaons using beam date identified through the $\rho$ and $\phi$ reaction at $[3.5,4] \mathrm{GeV} / \mathrm{c}$ momentum selected from the black rectangles defined in Fig. 5.9. The average photon yield per track for pion and kaons is 31 and 39 respectively. It was expected that both kaons and pions should have same photon yield. However, it was recognized that kaons have significantly higher photon yield compared to pions for not only beam data but also for simulation see Fig. 5.16b. The photon yield for simulated pion and kaons is 47 and 61 respectively. A 2-dimensions photon yield maps for beam data and simulation are illustrated in Fig. 5.17, the weight corresponds to the mean value of the histogram for each bin.

Therefore, more investigations were performed to understand this effect which could be a result of a potential background photons associated with $\phi$ calibration channel. A dedicated study was carried out by calculating the photon yield for events that have a single track at the DIRC wall. A significant reduction on the photon yield was observed, as using single-track events on the DIRC wall suppresses the number of background photons arises from non-related tracks (see Fig. 5.18). 

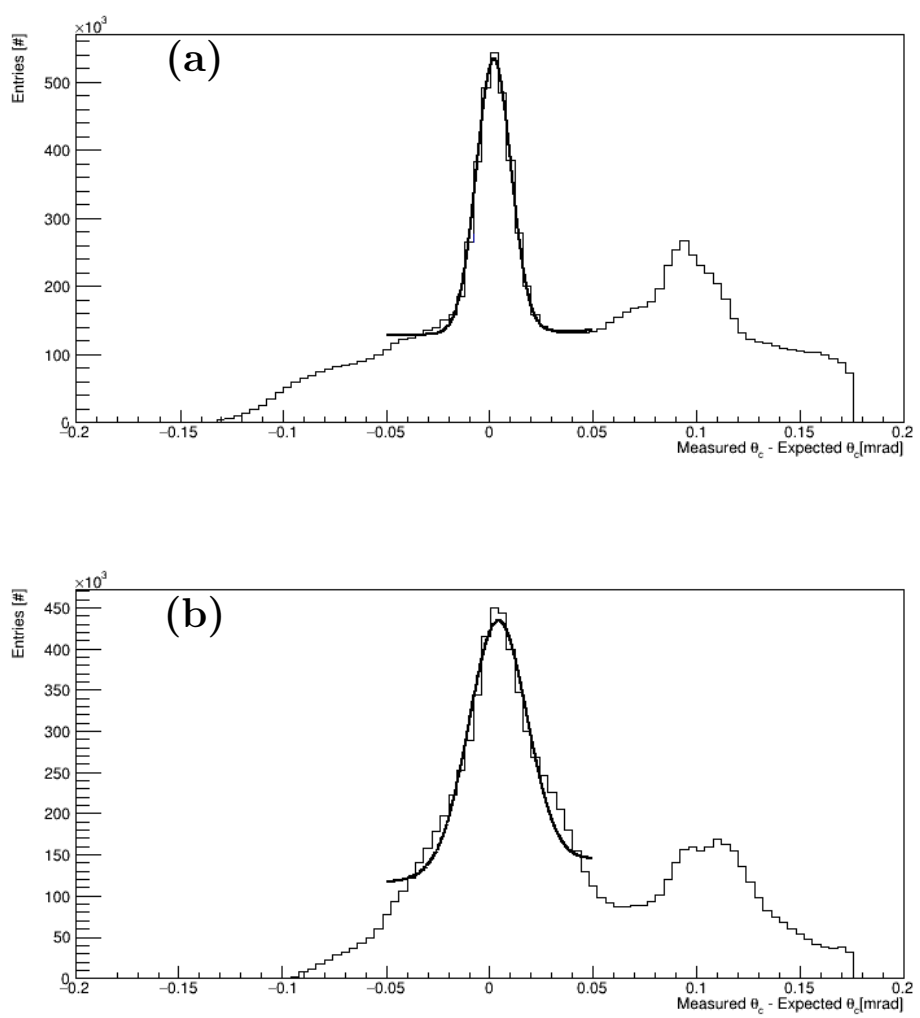

Figure 5.14: Difference between the measured and expected Cherenkov angle per photon, for kinematically identified pions beam data at $[3,3.5] \mathrm{GeV} / \mathrm{c}$ momentum, at the center of bar number 32 (a) at the center of bar number 24 (b), the peaks fitted with Gaussian plus second order polynomial function.

On the other hand, by comparison the beam data with the simulation, it was found that photon yield in simulation has more photons compared to the beam data see (Fig. 5.18). Several factors contribute to the disagreement between simulation and beam data such as a chemical corrosion on the mirrors in the optical box was observed after a few months from the first commissioning. It was reported that a chemical corrosion was developed on the reflective surfaces of the mirrors. The water quality was affected by the corrosion dust. To avoid this at the second commissioning run, the mirrors were covered by a glass and the gaps filled with a silicon to isolate the mirror from the surrounding water. However, there was water leakage, and corrosion was noticed after the second run. Another factors that could have contributed to the disagreement with simulation such as potential bar imperfections from either aging or from the transport and the quality of the optical couplings [52].

Furthermore, it can be seen that bar number 35 has a significantly lower photon yield compared to the neighbouring bars. This observation is not surprising because the same bar had shown a similarly low photon yield during BaBar operation [66]. This photon loss was caused by a water leak in this bar box, observed immediately after the installation of the BaBar DIRC in 1999, which caused pollutants in the water and bar box to be deposited on the sides of bar 35 . 

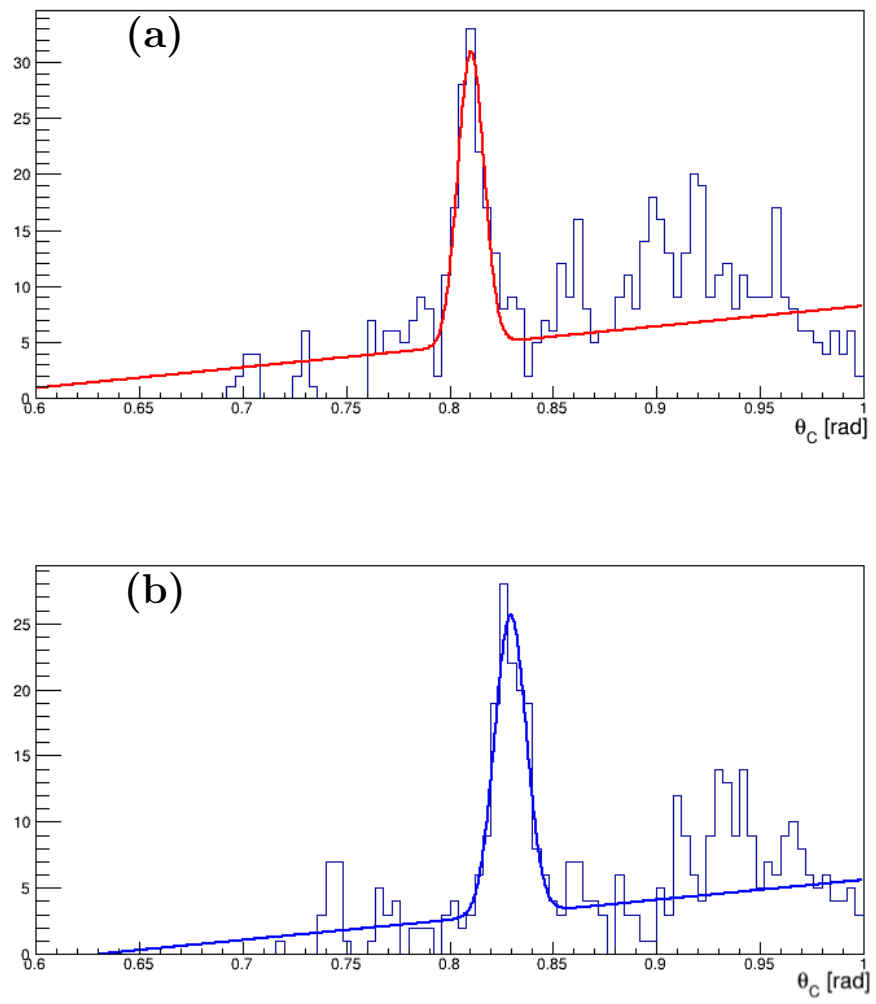

Figure 5.15: Example of the reconstructed Cherenkov angle per photon for single track beam data at $3 \mathrm{GeV} / \mathrm{c}$ for kinematic identified kaons (a) and pions (b)

\subsubsection{PID performance}

The PID performance can be evaluated using the log-likelihood differences for the kaons/pions by fitting the distributions with a Gaussian function, then calculating the separation power. The separation power for Gaussian likelihood distributions can be defined as the absolute value of the difference of the two mean values $\left(\mu_{1}, \mu_{2}\right)$ in units of the average of the two standard deviations $\left(\sigma_{1}, \sigma_{2}\right)$ :

$$
N_{\text {sep }}=\frac{\left|\mu_{1}-\mu_{2}\right|}{0.5\left(\sigma_{1}+\sigma_{2}\right)}
$$

Figure 5.19a shows the log-likelihood difference distribution for the kinematically identified kaon/pion at $[3,3.5] \mathrm{GeV} / \mathrm{c}$ momentum, defined by the tracks located in the black boxes shown in Fig. 5.9. The $\pi / \mathrm{K}$ separation power is $2.62 \pm 0.2$ s.d., such a performance is at least $25 \%$ less than the Geant expectations. After applying the Cherenkov angle correction the achieved $\pi / \mathrm{K}$ separation power is $2.2 .71 \pm 0.2$ standard deviations. Further studies were carried out to perform better bar boxes alignment corrections. The following subsection introduce description of misalignment mitigation methods.

\section{Alignment and Calibration}

Once the BABAR DIRC bar boxes were installed at Hall D, a rough alignment survey had carried out. Since the beam data performance is significantly below simulation predictions. one potential cause of this discrepancy arises from a residual 

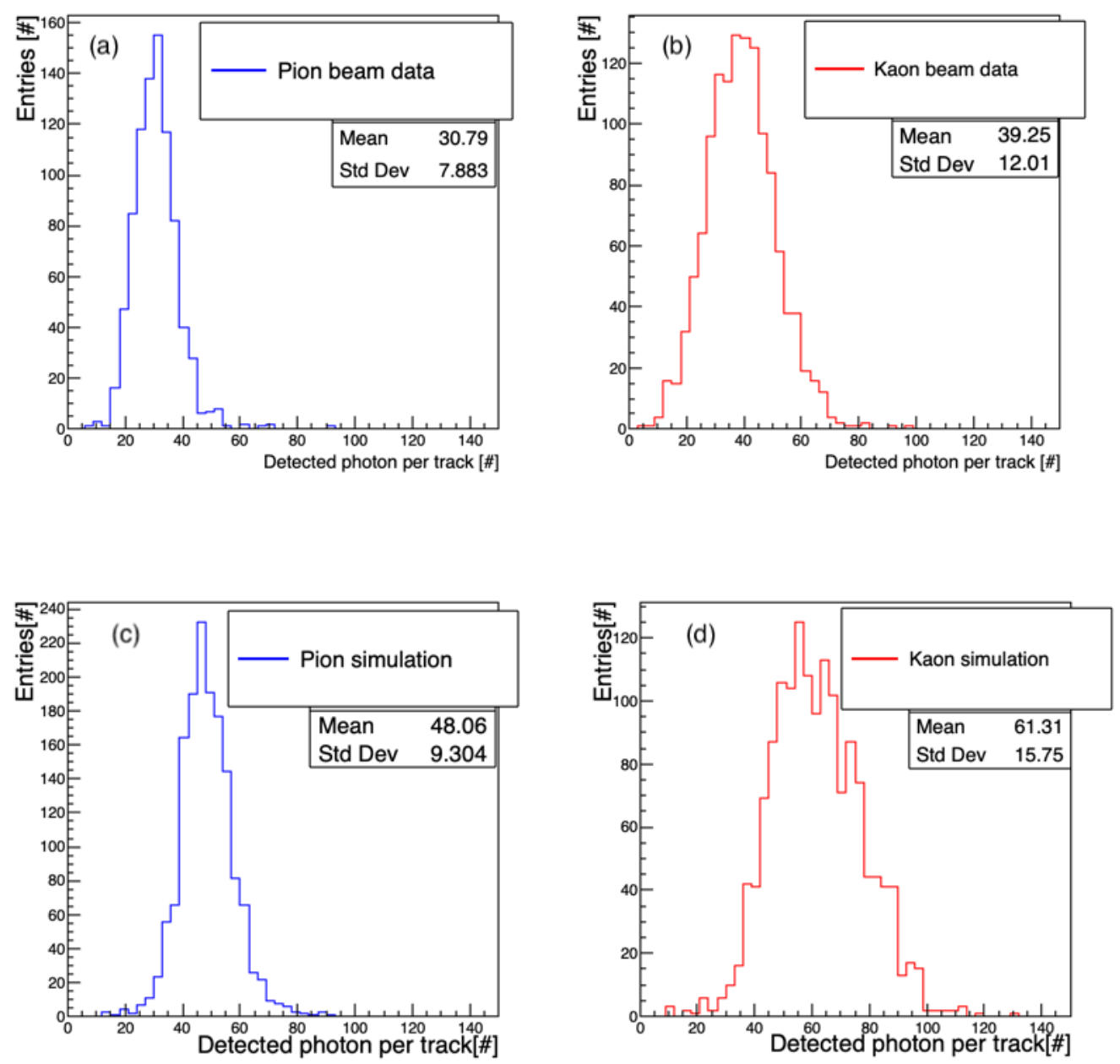

Figure 5.16: Distribution of the photon yield per particle for kinematically identified reaction selected from the black rectangles defined in Fig. 5.9, using beam data (a) kaons and (b) pions, and simulation in (c) kaons and (d) pions.

misalignment in the beam data or the LUT is not matching the detector precisely. Several observable can be used to perform further alignment, ideally the pion/kaon separation is the most sensitive quantities for such a study. However, since the calibration samples provide statistically limited kaons compared to pions. The single photon resolution (SPR) using pion data was primarily used as an observable for finding the optimum track momentum vector correction.

The correction was performed by rotating the pion track direction against the polar and the azimuthal angles and monitor the single photon resolution. Figure 5.20 shows the single photon resolution (SPR) map as a function of the polar and the azimuthal angles rotation for the north optical box (top) and south optical box (bottom). The color scale corresponds to the SPR value in units of mrad using pions beam data at $\mathrm{GeV} / \mathrm{c}$ momentum. The black dot corresponds to the selected correction value. This correction will implicitly be translated into improvements in the pion/kaon separation power. 


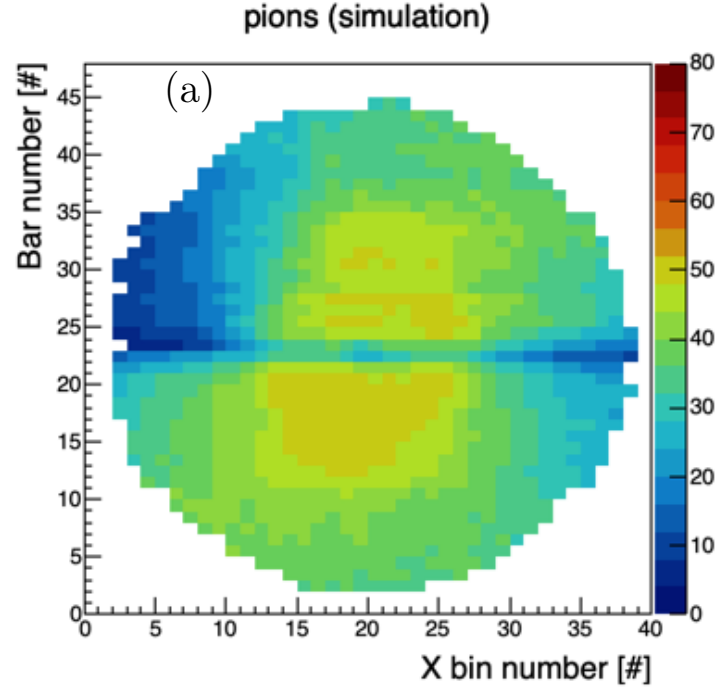

kaons (simulation)

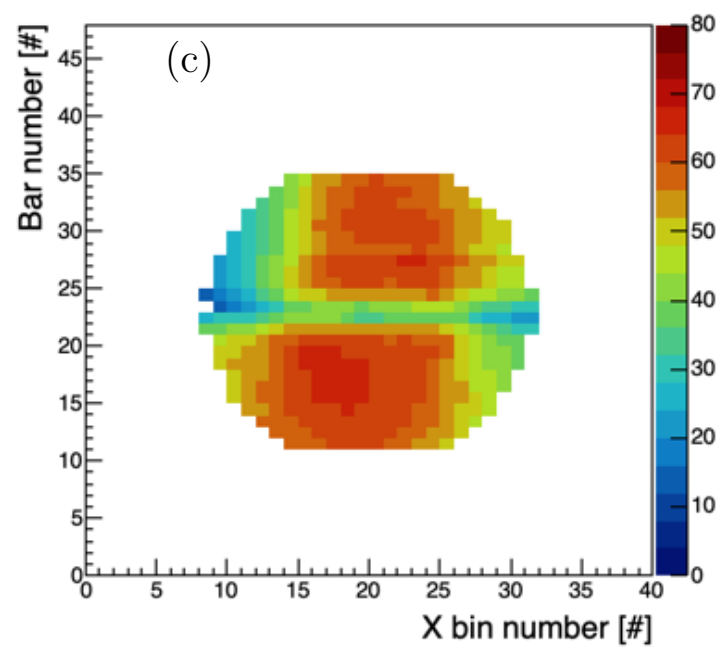

pions (beam data)

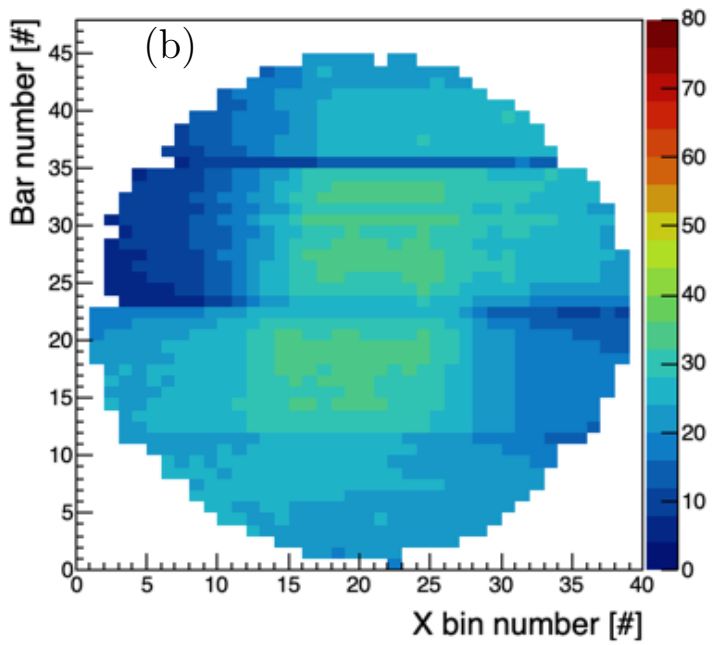

kaons (beam data))

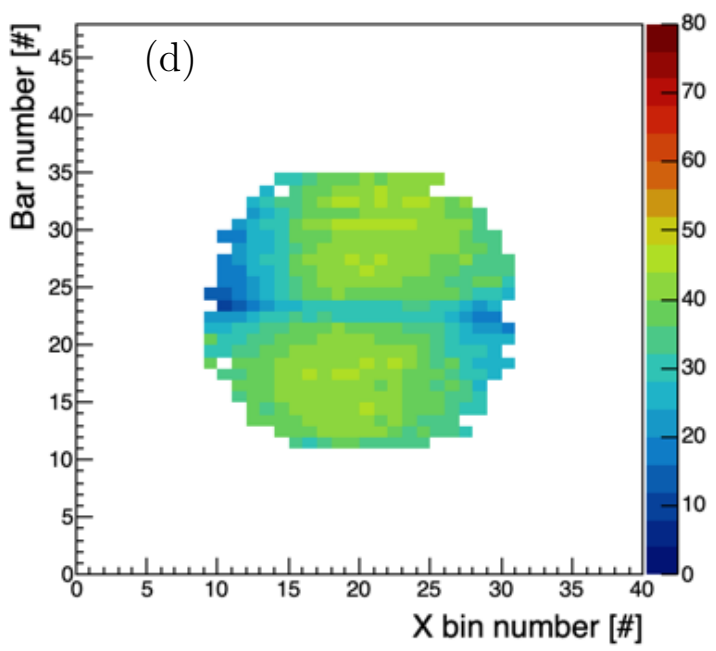

Figure 5.17: Mean value of the photon yield per particle at $[3,3.5] \mathrm{GeV} / \mathrm{c}$ momentum for pions on (a) simulation and (b) beam data, and kaons in (c) simulation and (d) beam data. 


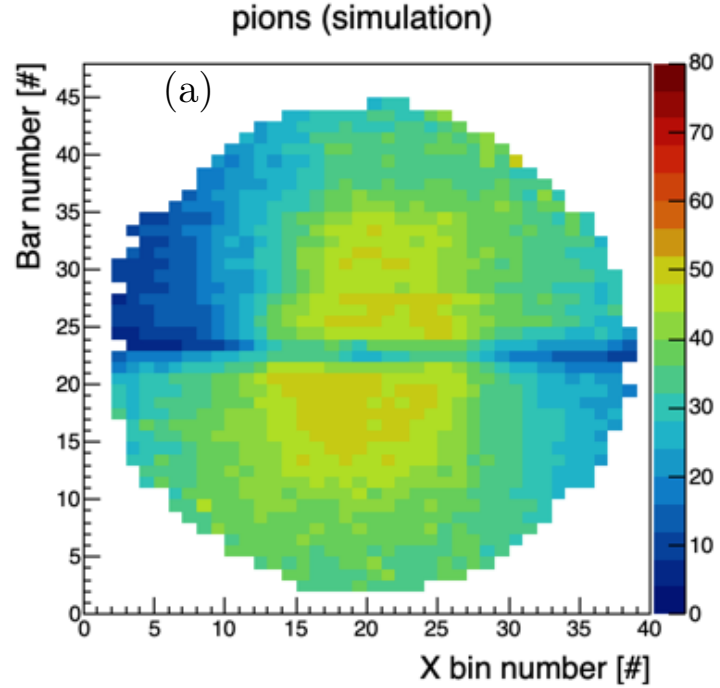

kaons (simulation)

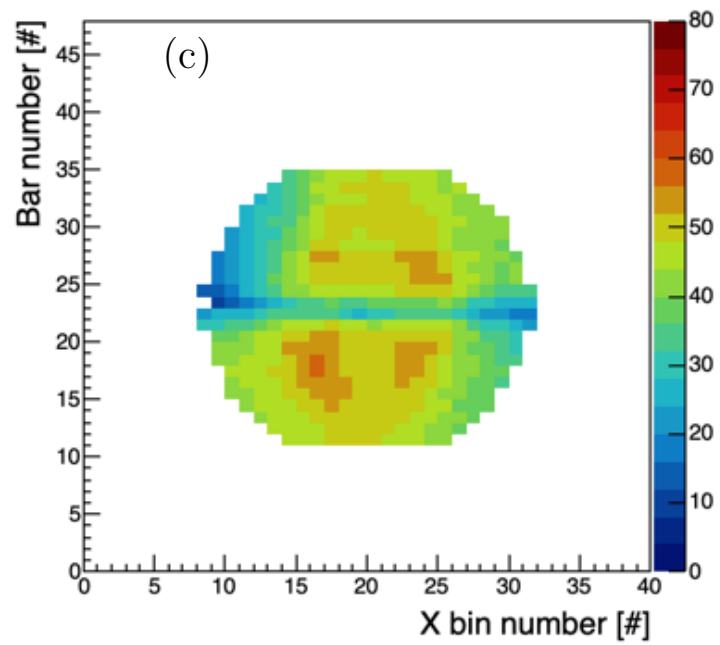

pions (beam data)

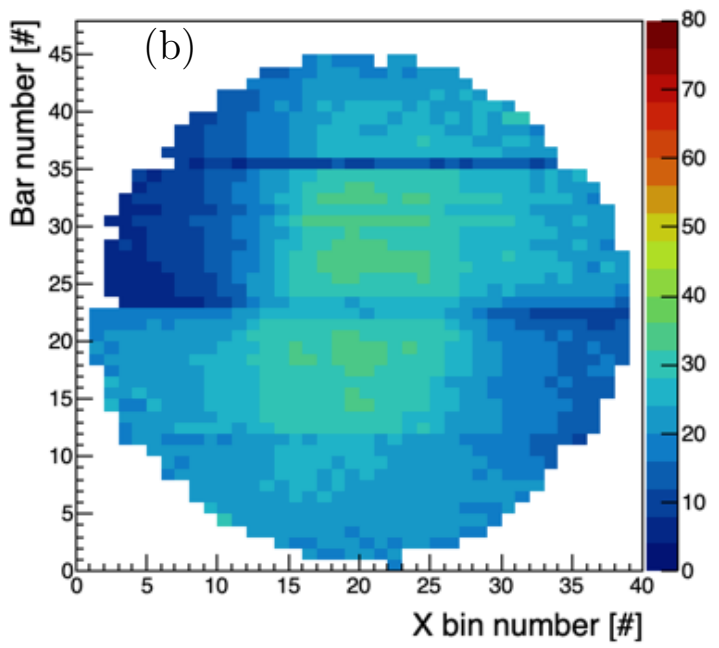

kaons (beam data)

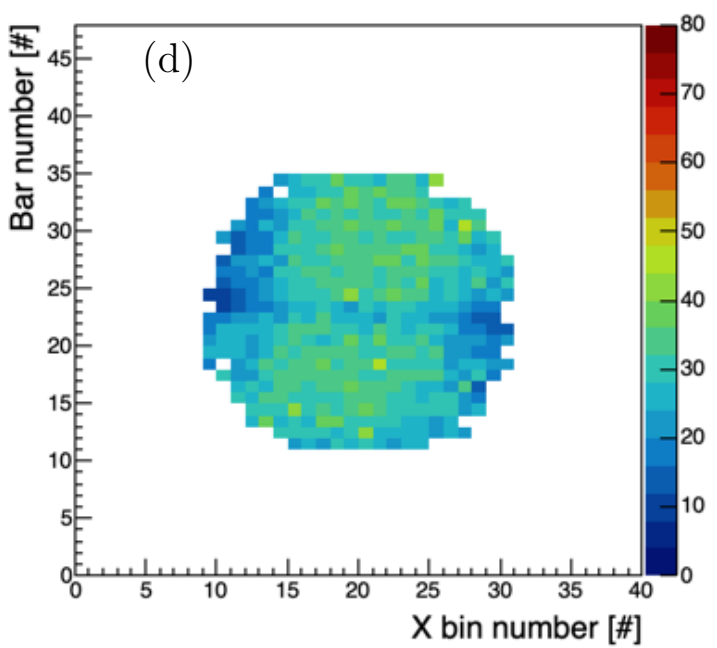

Figure 5.18: Mean value of the photon yield per particle at $[3,3.5] \mathrm{GeV} / \mathrm{c}$ momentum for pions on (a) simulation and (b) beam data, and kaons in (c) simulation and (d) beam data. using events that have single track at the DIRC wall. 
(a) Beam data without correction

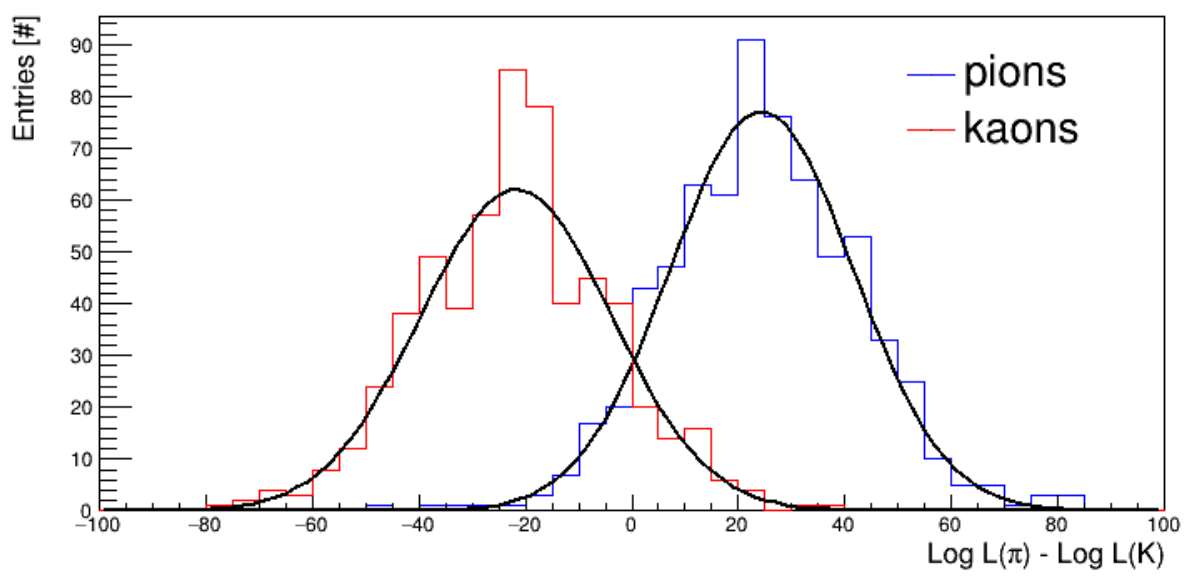

(b) Beam data after Cherenkov angle correction

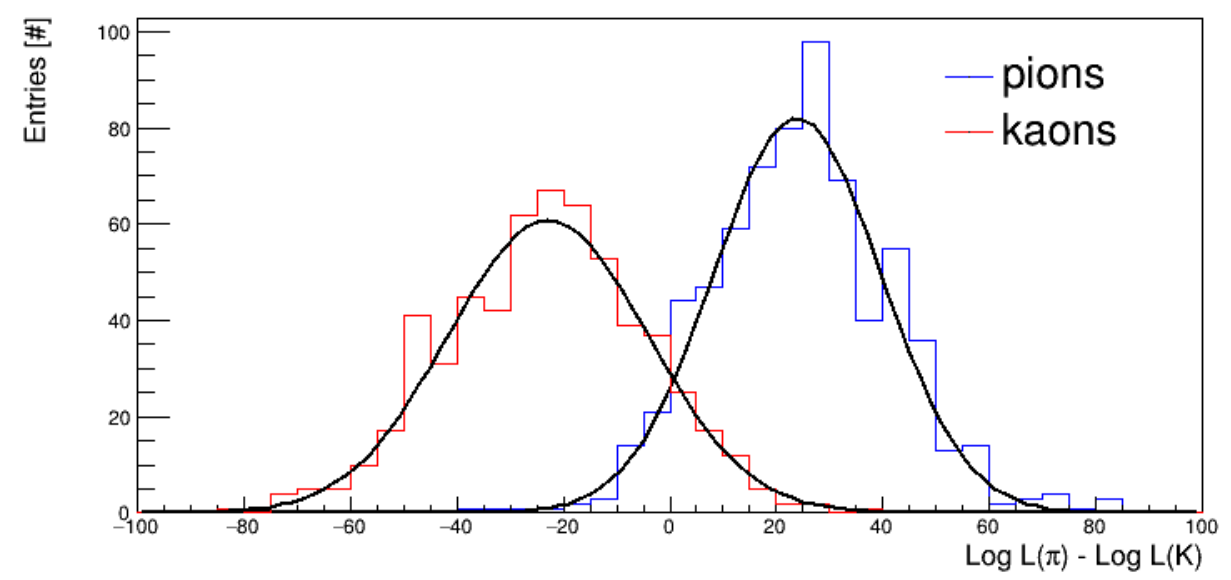

Figure 5.19: $\pi / \mathrm{K}$ log-likelihood difference distributions for the kaon hypothesis (red) and pion hypothesis (blue) beam events as result of the geometrical reconstruction at $[3,3.5] \mathrm{GeV} / \mathrm{c}$ momentum, defined by the tracks located in the black boxes shown in Fig. 5.9. The $\pi / \mathrm{K}$ separation power value is $2.62 \pm 0.2 \mathrm{~s} . \mathrm{d}$. before applying the Cherenkov angle correction (a), and $2.71 \pm 0.2$ s.d. after applying the Cherenkov angle correction (b)

Example of the log-likelihood differences distribution (after applying bar boxes alignment correction) of the kaon/pion hypothesis for beam data at $[3,3.5] \mathrm{GeV} / \mathrm{c}$ momentum is shown in Fig. 5.21 and the $\pi / \mathrm{K}$ separation power value is $2.9 \pm 0.2$ standard deviation. The $\pi / \mathrm{K}$ separation is improved after applying the correction, however, it is at least $20 \%$ less than simulation predictions.

The $\pi / \mathrm{K}$ separation power map over the DIRC wall is shown in Fig. 5.22, with two momentum bins. The distribution roughly follows the typical shape of the photon yield map on the DIRC wall. The resulting separation power (commissioning beam data) at $[3,3.5] \mathrm{GeV} / \mathrm{c}$ momentum shows few bar number/X bin points that are around or slightly above 3 s.d. but most of the wall shows values much below 3 standard deviations. However, the simulation shows better performance by approximately $1 \sigma$. This disagreement between simulation and beam data is under 
(a) Track direction correction north optical box

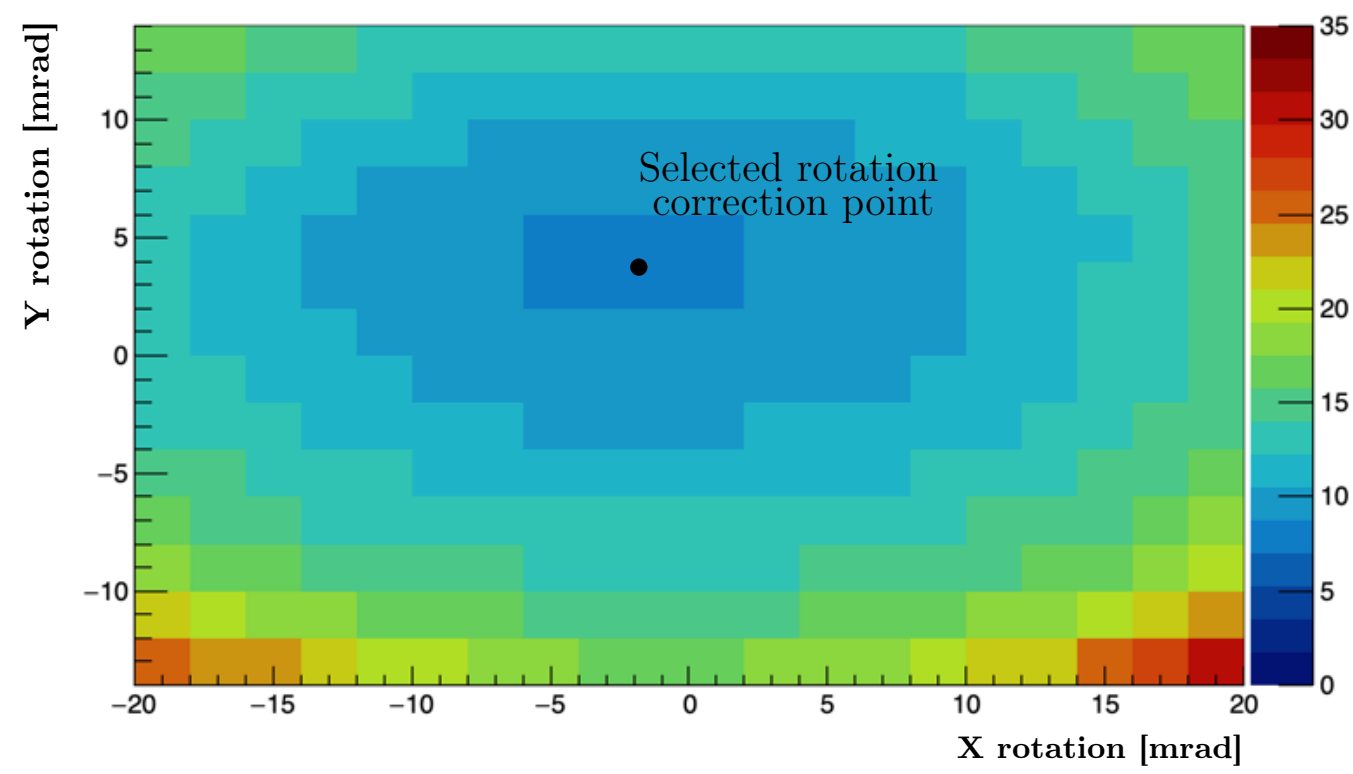

(b) Track direction correction south optical box

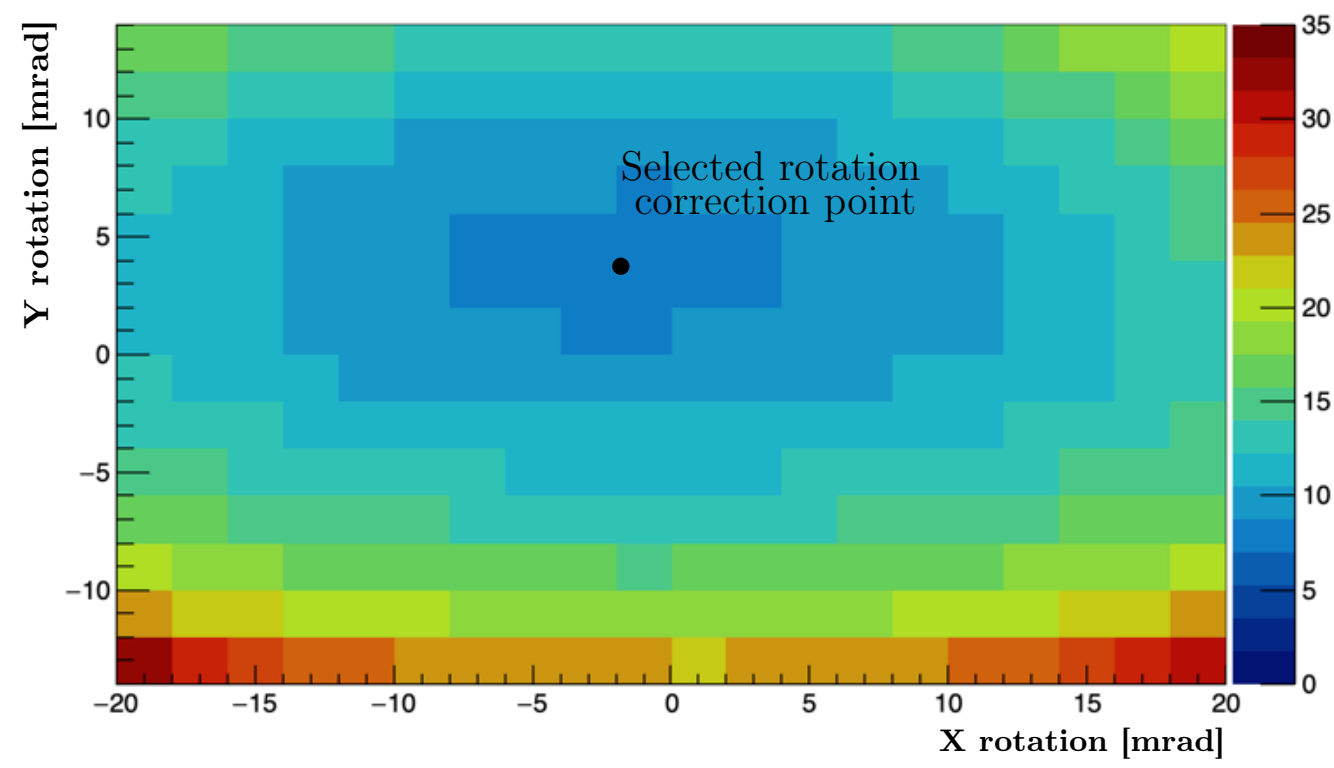

Figure 5.20: Single Photon Resolution (SPR) map using beam data as a function of the pion momentum rotations, in the momentum range of [3,3.5] $\mathrm{GeV} / \mathrm{c}$. The color scale corresponds to the SPR value, for north optical box (a) and south optical box (b). The black dots indicate the selected rotation value. 
After adding track direction correction

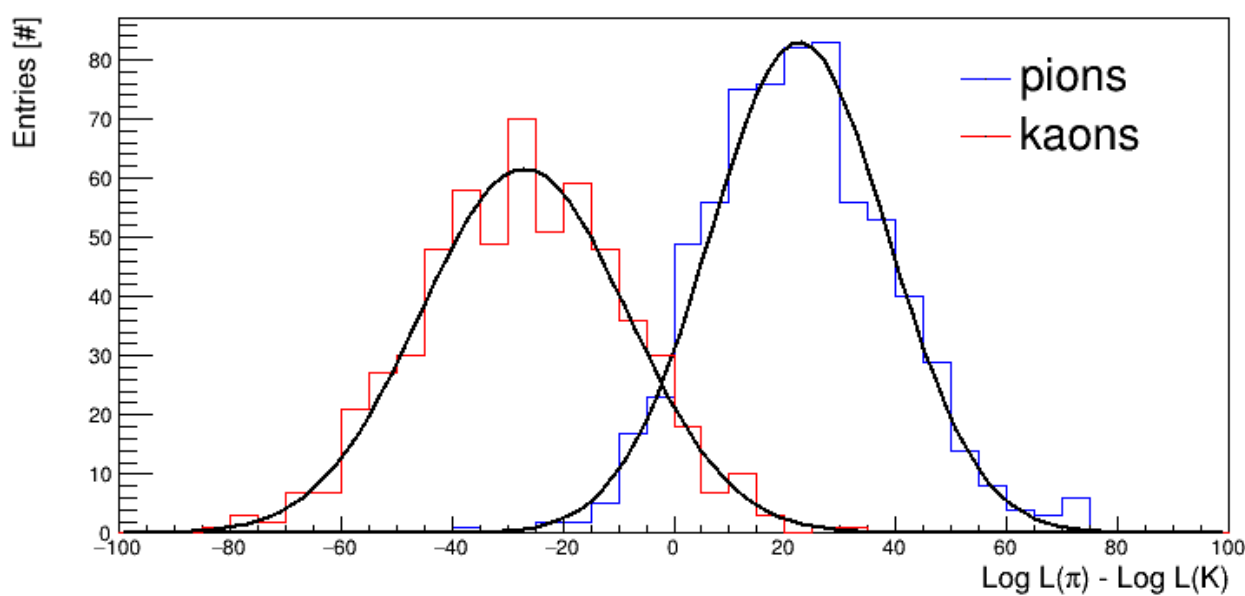

Figure 5.21: $\pi / \mathrm{K}$ log-likelihood difference distributions for the kaon hypothesis (red) and pion hypothesis (blue) beam events as result of the geometrical reconstruction at $[3,3.5] \mathrm{GeV} / \mathrm{c}$ momentum, defined by the tracks located in the black boxes shown in Fig. 5.9. The $\pi / \mathrm{K}$ separation power value is $2.9 \pm 0.2$ s.d. after applying the momentum correction.

investigation. One of the possible reasons is the relatively low photon yield per track for the commissioning data. Given that, for the PANDA Barrel DIRC test beam at CERN PS, an excellent agreement between beam data simulation was observed, which manifests to exclude the simulation from being the reason for this disagreement. To determine how the photon yield difference affects the pion/kaon separation, a simulation study was carried out by randomly removing photons from the simulation sample to match the photon yield of the beam data sample. Simulation results in Fig. 5.23 show that separation power decreased by about $30 \%$ bringing it closer to that observed in the data. The current alignment and calibration efforts enables us to keep only small solution fragments, which leads to a plenty of room for future improvements. Such as chromatic dispersion correction, LUT fine tuning and testing more physics reactions.

\section{Cherenkov Angle Data PDF}

The Cherenkov data PDF PID method was applied to the PANDA Barrel DIRC test beam data, it was shown that the separation power results are less dependent on detailed alignment and calibration see 4.8.5. For the GlueX DIRC, the collected commissioning data sample during December 2019 is statistically limited. Therefore, the method was tested using one third of the 2020 dataset. Even then, the statistics are not enough to cover a large part of the DIRC wall, instead the method is applied only to a small region on the DIRC wall, in particular the central region of bar number 34 to select a region with marginally sufficient sample size. On the meanwhile the PDF sample size was enlarged by switching from Cherenkov angle to the difference between measured and expected Cherenkov angle, opening up the momentum range for the PDFs. Figure 5.24 shows pion and kaon PDFs for one pixel. A clear peak around zero can be seen, in addition to combinatorial reconstruction background. Figure 5.25 shows the log-likelihood difference distributions for pions and kaons using beam events at $[3.5,4] \mathrm{GeV} / \mathrm{c}$ momentum for the center of 
$[3,3.5] \mathrm{GeV} / \mathrm{c}$ Simulation

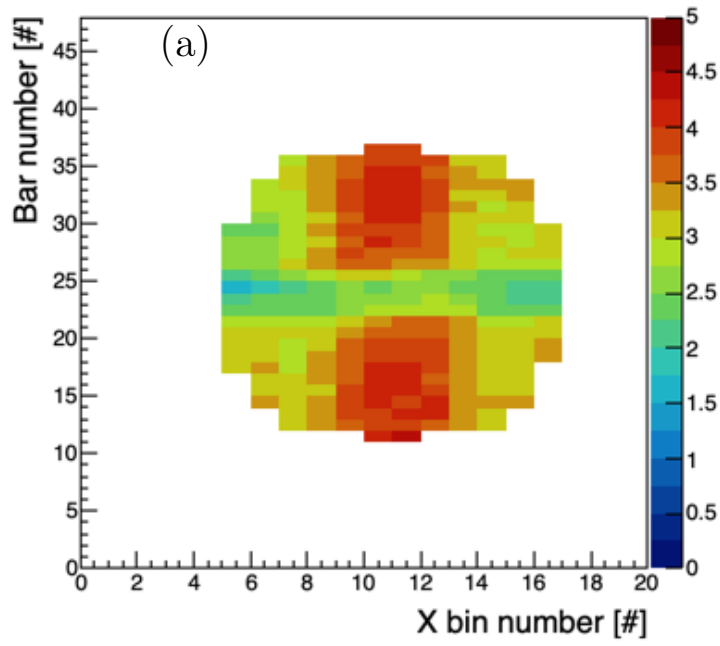

$[3.5,4] \mathrm{GeV} / \mathrm{c}$ Simulation

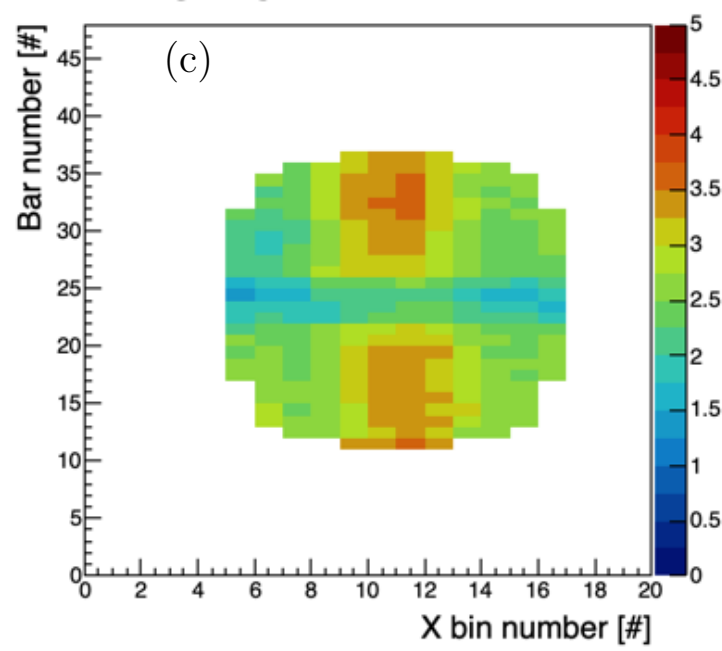

$[3,3.5] \mathrm{GeV} / \mathrm{c}$ Beam Data

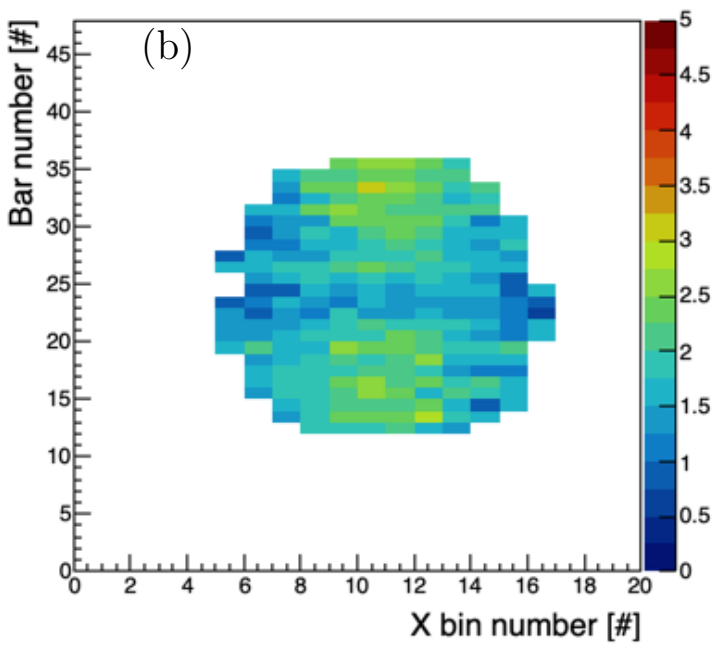

$[3.5,4] \mathrm{GeV} / \mathrm{c}$ Beam Data

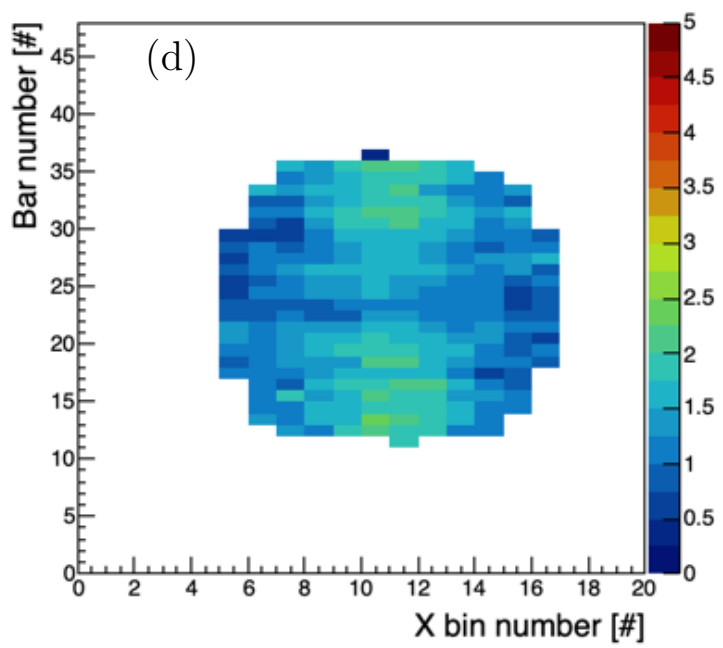

Figure 5.22: Separation power maps for pion/kaon identified through the $\rho$ and $\phi$ reactions. The color scale corresponds to the separation value in units of standard deviations for simulation (left) and beam data (right) for the momentum range $[3,3.5] \mathrm{GeV} / \mathrm{c}$ (top) and [3.5,4.0] GeV/c (bottom). 

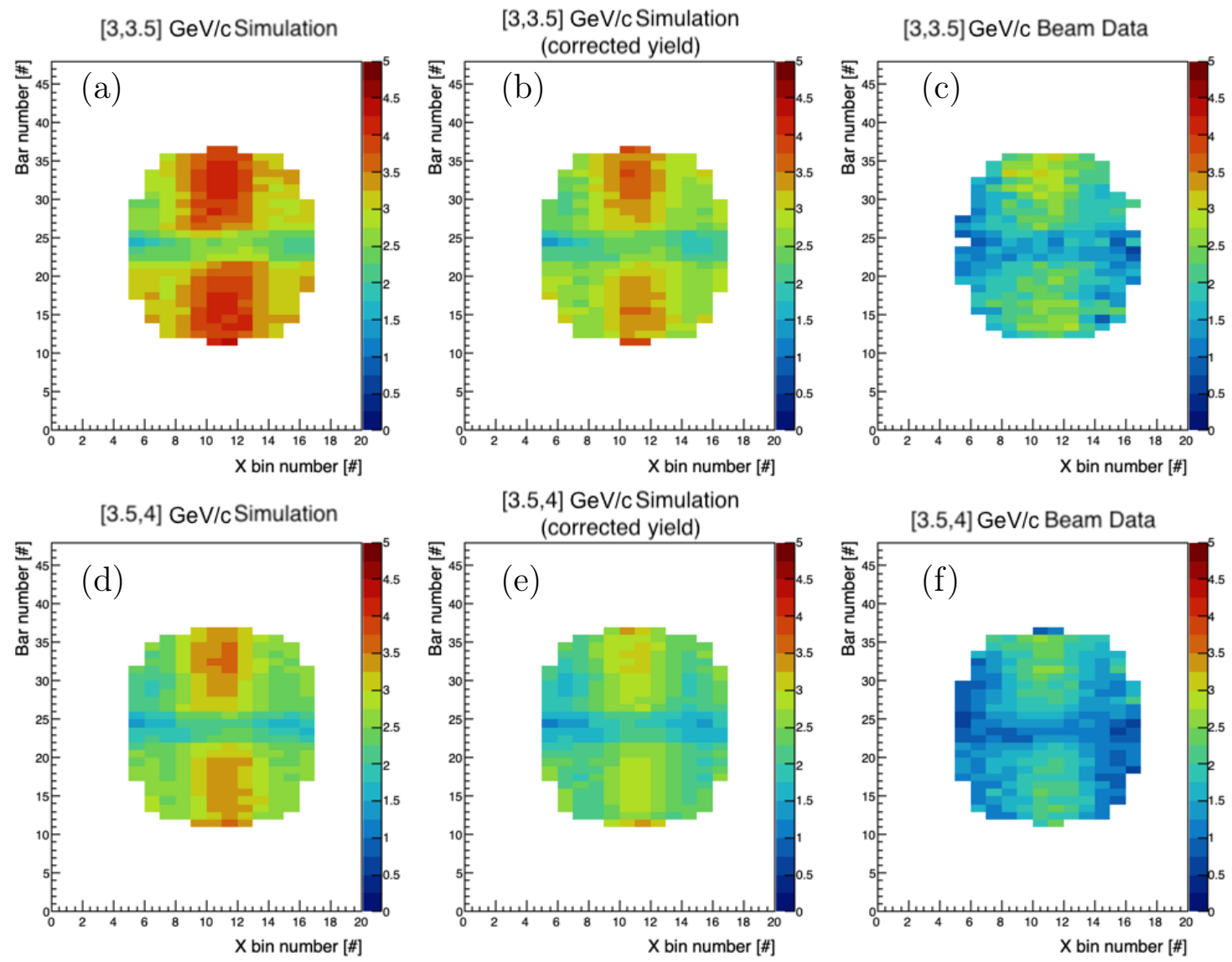

Figure 5.23: Separation power maps for pion/kaon identified through the $\rho$ and $\phi$ reactions. The color scale corresponds to the separation value in units of standard deviations for simulation (left), simulation after yield correction (middle) and beam data (right) for the momentum range $[3,3.5] \mathrm{GeV} / \mathrm{c}$ (top) and $[3.5,4.0] \mathrm{GeV} / \mathrm{c}$ (bottom). 
the bar 34. The observed separation power value is $2.25 \pm 0.2$ s.d. using the standard geometrical reconstruction and $2.3 \pm 0.2$ s.d. using the Cherenkov data PDF PID method. The obtained performance is consistent with the standard geometrical reconstruction without the need for the complex detector alignment procedure discussed above. However, the preliminary Cherenkov data PDFs are created using a small commissioning dataset that shows promising results, and further improvements are expected once enough statistics are obtained.
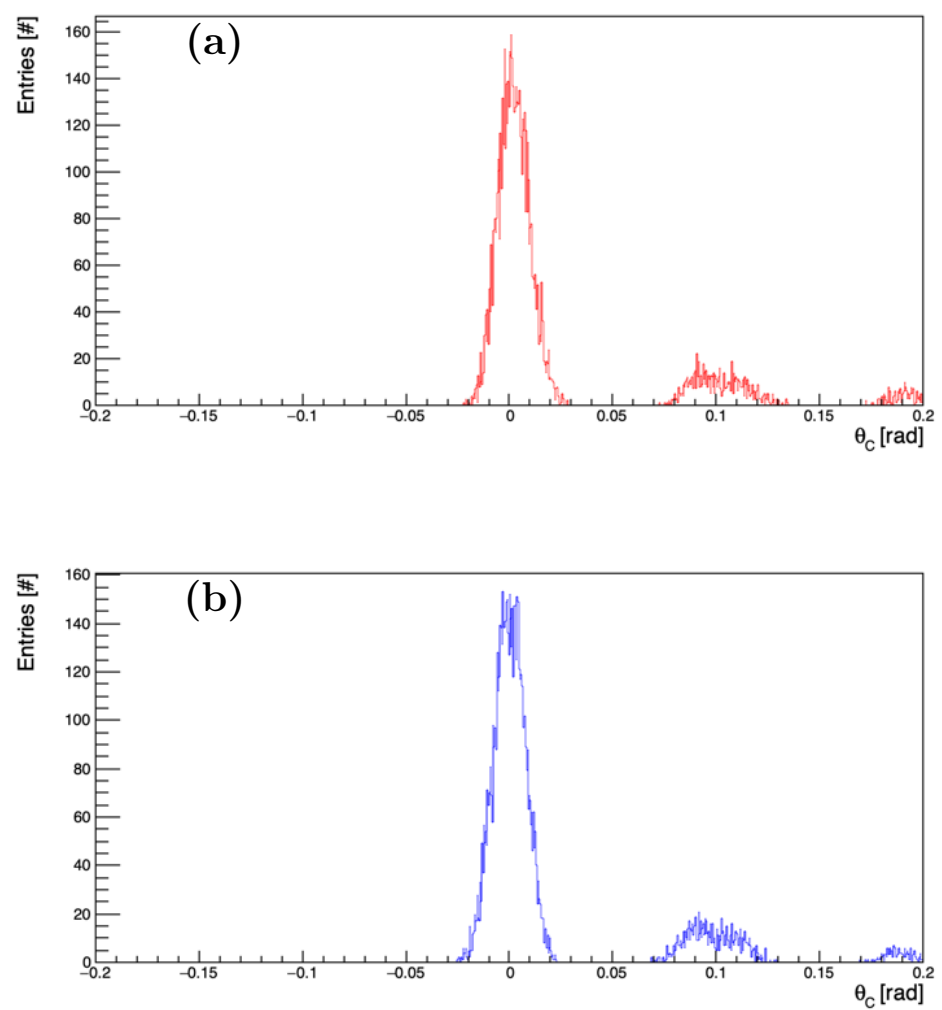

Figure 5.24: The difference between the expected and measured Cherenkov angle perfoton for a single pixel in beam data using the track momentum range between $1.5-4 \mathrm{GeV} / \mathrm{c}$ for kaons (a), for pions (b). 

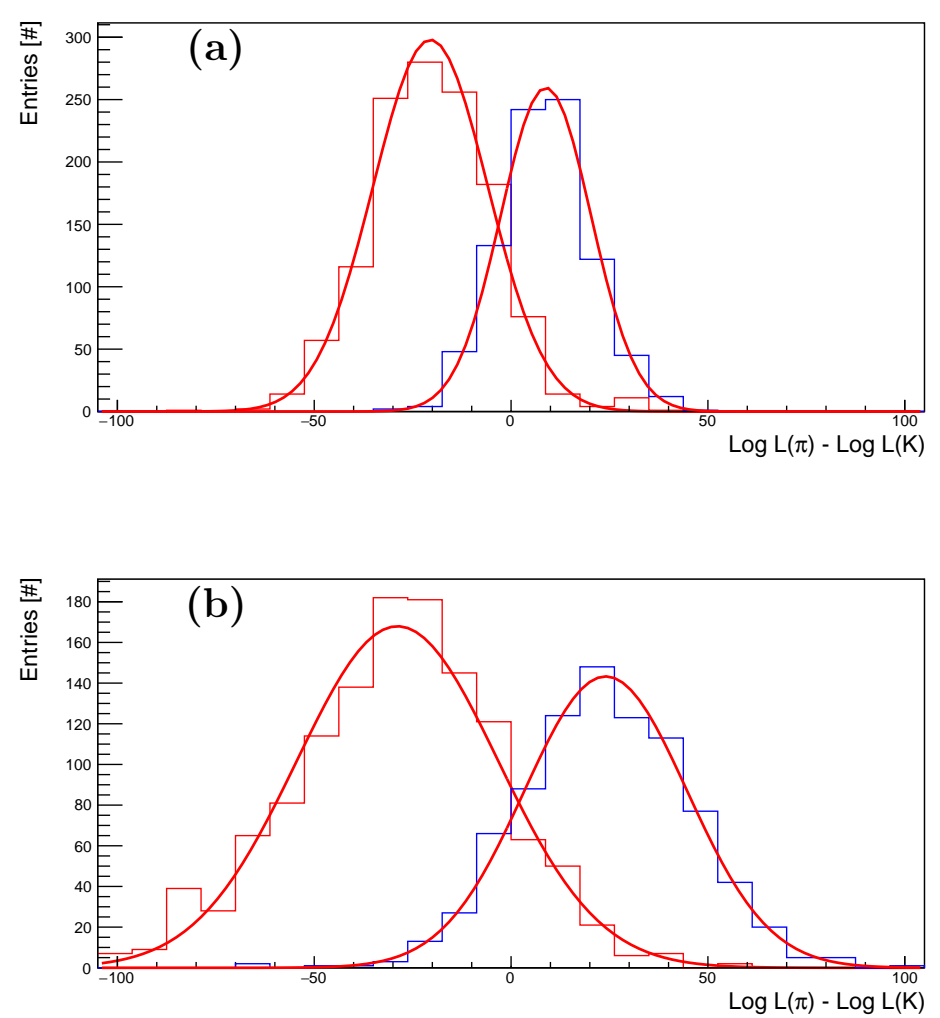

Figure 5.25: $\pi / \mathrm{K}$ log-likelihood difference distributions for the kaon hypothesis (red) and pion hypothesis (blue) for beam events at $[3.5,4] \mathrm{GeV} / \mathrm{c}$ momentum, for bar number 34 at the center of the bar. The $\pi / \mathrm{K}$ separation power value is $2.25 \pm 0.2$ standard deviations using the standard geometrical reconstruction (a), and $2.3 \pm$ 0.2 standard deviations using the Cherenkov data PDF PID method (b)

\subsubsection{Summary}

The ability to separate final state kaons from pions is important for the physics potential of the GlueX experiment. To enhance the particle identification capabilities of GlueX, a DIRC detector was installed in the forward region of the experiment. In December 2019, the complete GlueX DIRC detector was installed utilizing four bar boxes from the decommissioned BABAR DIRC coupled to two new Optical Boxes. The integration of the DIRC system marked the beginning of the GlueX experiment Phase II running. An LED-based calibration system is used to remove the time offsets between the pixels, where a timing resolution of $0.7 \mathrm{~ns}$ is achieved. Two physics channels that include the decay of $\rho$ and $\phi$ have been selected to study the detector performance providing a pure final state samples of $\pi^{ \pm}$and $\mathrm{K}^{ \pm}$.

The PANDA Barrel DIRC simulations and reconstruction methods have been deployed to the GlueX software, to apply lessons learned to PANDA, as part of the FAIR Phase-0 program. The latest results obtained for a small commissioning dataset already show very good matching with Monte-Carlo simulations in terms of the observed hit patterns and the single Cherenkov angle resolution. However, the photon yield is below the Geant4 predictions. It is important to recall that, for the PANDA Barrel DIRC test beam at CERN PS, an excellent agreement between beam 
data simulation was observed, which manifests to exclude the simulation from being overestimating the photon yield. On the other hand, the $\pi / \mathrm{K}$ separation power using the geometrical reconstruction was applied to the available commissioning dataset at $[3,3.5] \mathrm{GeV} / \mathrm{c}$ momentum range. The results show few regions on the DIRC wall are around or slightly above 3 s.d., but most of the wall shows values much below 3 s.d. Given that the limited calibration sample size adds a major challenge to apply the Cherenkov angle data PDF method with full power. This statistical limitation creates also an obstacle to use the time-imaging PID methods.

However, the achieved results using the geometrical reconstruction are very encouraging, and it is expected that the GlueX DIRC performance will be improved, once an enough statistics with advanced detector alignment and calibration are applied. 


\section{Chapter 6}

\section{Conclusion and Outlook}

The PANDA experiment will be one of the key experiments at the Facility for Antiproton and Ion Research (FAIR) in Germany near Darmstadt. It is designed to study hadronic interactions using cooled antiproton beams with momenta up to 15 $\mathrm{GeV} / \mathrm{c}$ interacting with various internal targets. For the success of the PANDA physics program, robust particle identification for a wide momentum range and the full solid angle is mandatory. For this purpose, the hadronic particle identification in the barrel region of the PANDA target spectrometer will be delivered by a DIRC counter. The Barrel DIRC is designed to cleanly separate kaons with momenta up to $3.5 \mathrm{GeV} / \mathrm{c}$ from a large pionic background, with a minimum $\pi / \mathrm{K}$ separation power of 3 standard deviations. The design of the PANDA Barrel DIRC was inspired by the successful BABAR DIRC counter with several key improvements, such as fast timing, lens focusing, and a compact expansion volume.

The prototype program was established to show if the predicted performance in the simulation can be confirmed experimentally. The near final-design of the PANDA Barrel DIRC was implemented in the prototype and tested with hadronic particle beams in 2018 to validate the design and different reconstruction and PID methods. The prototype was placed into the mixed hadron beam in the T9 zone at the CERN PS. The particle beam was primarily composed of pions and protons, which meant that the direct evaluation of $\pi / \mathrm{K}$ separation was not possible. Instead, the PID performance was evaluated using pions and protons. The Cherenkov angle difference for pions and protons at $7 \mathrm{GeV} / \mathrm{c}$ is approximately the same as for pions and kaons at $3.5 \mathrm{GeV} / \mathrm{c}$, the upper limit of the PID performance range of the PANDA Barrel DIRC. Detailed Monte Carlo simulation of the prototype, including the expected technical characteristics of the MCP-PMTs, lens, optical coupling material, bar and prism dimensions, and readout timing resolution, was carried out to compare the performance to test beam results. The performance of the prototype was characterized in terms of the photon yield, the single photon Cherenkov angle resolution, and $\pi / \mathrm{p}$ separation power.

The reconstruction and PID methods are the heart of the DIRC detector. Their validation using particle beams at CERN PS, physics events at the GlueX experiment at JLab, and Geant4 simulation form the core of this thesis. Several reconstruction algorithm approaches were developed, tested, and optimized using beam particles, kinematically identified physics channels, and Geant4 simulations. 
The geometrical reconstruction is a fast algorithm that delivers measurements of the single photon Cherenkov angle resolution, the Cherenkov angle of the particle, and the photon yield, which are used to perform the PID. The Cherenkov data PDF is a new method developed for this thesis, based on the geometric reconstruction, providing less dependence on proper alignment, achieving comparable performance with the standard geometric reconstruction. The time imaging PID method using data PDFs has superior performance to the geometrical reconstruction. However, it requires large storage resources for the PDFs for each angle, position, momentum, and particle type. Time imaging using analytically calculated PDFs would solve these issues but have not been implemented for the Barrel DIRC yet and it remains to be seen if the PID performance is competitive with the results from the standard time-based approach. The test beam results show $\pi / \mathrm{p}$ separation values of up to $5.0 \pm 0.2$ standard deviation (s.d.) at $7 \mathrm{GeV} / \mathrm{c}$, corresponding to $5.2 \pm 0.2$ s.d. $\pi / \mathrm{K}$ separation at $3.5 \mathrm{GeV} / \mathrm{c}$, using the time imaging PID method, exceeding the PANDA PID requirements at this angle. The analysis results of the prototype test at CERN were shown to be in excellent agreement with simulation, validating the PANDA Barrel DIRC Geant4 simulation.

The PANDA Barrel DIRC simulation and reconstruction methods were also applied to the GlueX framework environment, as part of the FAIR Phase-0 program. The initial results obtained for the available commissioning dataset show that the observed hit patterns and the single Cherenkov angle resolution are consistent with the expectation and Monte Carlo simulations. However, the photon yield and the $\pi / \mathrm{K}$ separation power using the geometrical reconstruction are significantly below the Geant4 predictions. The small size of the calibration samples prevents the use of the time imaging PID method and limits the application of the Cherenkov angle data PDF method with its full power. However, the obtained results for the geometrical reconstruction are very promising, with up to 3 s.d. $\pi / \mathrm{K}$ separation at a momentum of 3.0-3.5 GeV/c, it is expected that the GlueX DIRC performance will improve further once an advanced detector alignment and calibration are applied.

The comparison of the performance of the reconstruction and PID methods was an important part of the validation of the final PANDA Barrel DIRC design. It helped to pave the way to the series production of the Barrel DIRC components, currently underway. The selection of the optimal algorithms will be a critical aspect for the next-generation of DIRC counters, like the Barrel DIRC for the Electron-Ion Collider detector, which will use even better timing and position resolution to push the 3 s.d. $\pi / \mathrm{K}$ separation range to momenta of $6 \mathrm{GeV} / \mathrm{c}$ or higher. 


\section{Chapter 7}

\section{Ausführliche Zusammenfassung}

Das PANDA-Experiment an der internationalen Beschleunigeranlage Facility for Antiproton and Ion Research in Europe (FAIR) bei der GSI in Darmstadt wird ein breites Spektrum fundamentaler Fragen der Hadronen- und Kernphysik untersuchen. Es ist darauf ausgelegt, hadronische Wechselwirkungen unter Verwendung hochintensiver gekühlter Antiprotonenstrahlen mit Impulsen bis zu $15 \mathrm{GeV} / \mathrm{c}$ an einem internen stationären Target zu streuen und alle Teilchen/Ereignisse dieser Reaktionen zu messen. Der PANDA-Detektor ist so ausgelegt, dass er nahezu den kompletten Raumwinkel von $4 \pi$ um den Wechselwirkungspunkt abdeckt, mit mehreren hermetischen Schichten von Subdetektoren. Dabei werden ein Solenoid- und ein Dipolmagnet, mehrere Spurdetektoren, Teilchenidentifikationsdetektoren (PID), Kalorimeter und Myonendetektoren eingesetzt.

Um die Ziele der PANDA-Physik zu erreichen, ist eine exzellente Teilchenidentifikation für den ganzen Impulsbereich und den vollen Raumwinkel erforderlich. Zur hadronischen Teilchenidentifikation im zentralen Bereich des PANDA-Targetspektrometers wird ein DIRC-Zähler mit einer zylindrischen Form eingesetzt. Eine schematische Darstellung des PANDA Barrel DIRC-Detektors ist in Abb. 7.1 zu sehen.

Der Barrel DIRC deckt den Polarwinkelbereich $22^{\circ} \leq \theta \leq 140^{\circ}$ ab und wurde entwickelt um eine $\pi / \mathrm{K}$-Trennung von mindestens 3 Standardabweichungen (s.d.) für Impulse bis zu 3,5 GeV/c zu liefern. Abbildung 7.2 zeigt die Phasenraumverteilung für geladenen Kaonen als Funktion des Impulses und Polarwinkels. Der durch den PANDA Barrel DIRC abzudeckende Bereich ist mit einem gestrichelten Rechteck markiert. Das Design des PANDA Barrel DIRC wurde durch den erfolgreichen BABAR DIRC-Zähler inspiriert, mit einigen wichtigen Verbesserungen, wie höhere Zeitauflösung, Linsenfokussierung und einem kompakten Expansionsvolumen.

DIRC-Detektoren der nächsten Generation, wie der PANDA Barrel DIRC, mit verbesserten optischen Designs und besserer räumlicher und zeitlicher Auflösung, erfordern entsprechend fortschrittliche Rekonstruktions- und PID-Methoden. Im Rahmen dieser Doktorarbeit wurde die PID-Leistung von zwei DIRC-Zählern, dem Prototyp für den PANDA Barrel DIRC und dem GlueX DIRC-Detektor, untersucht und die Resultate verschiedener Rekonstruktionsalgorithmen, geometrischer und zeitabbildender Methoden, mit Simulationen verglichen. 


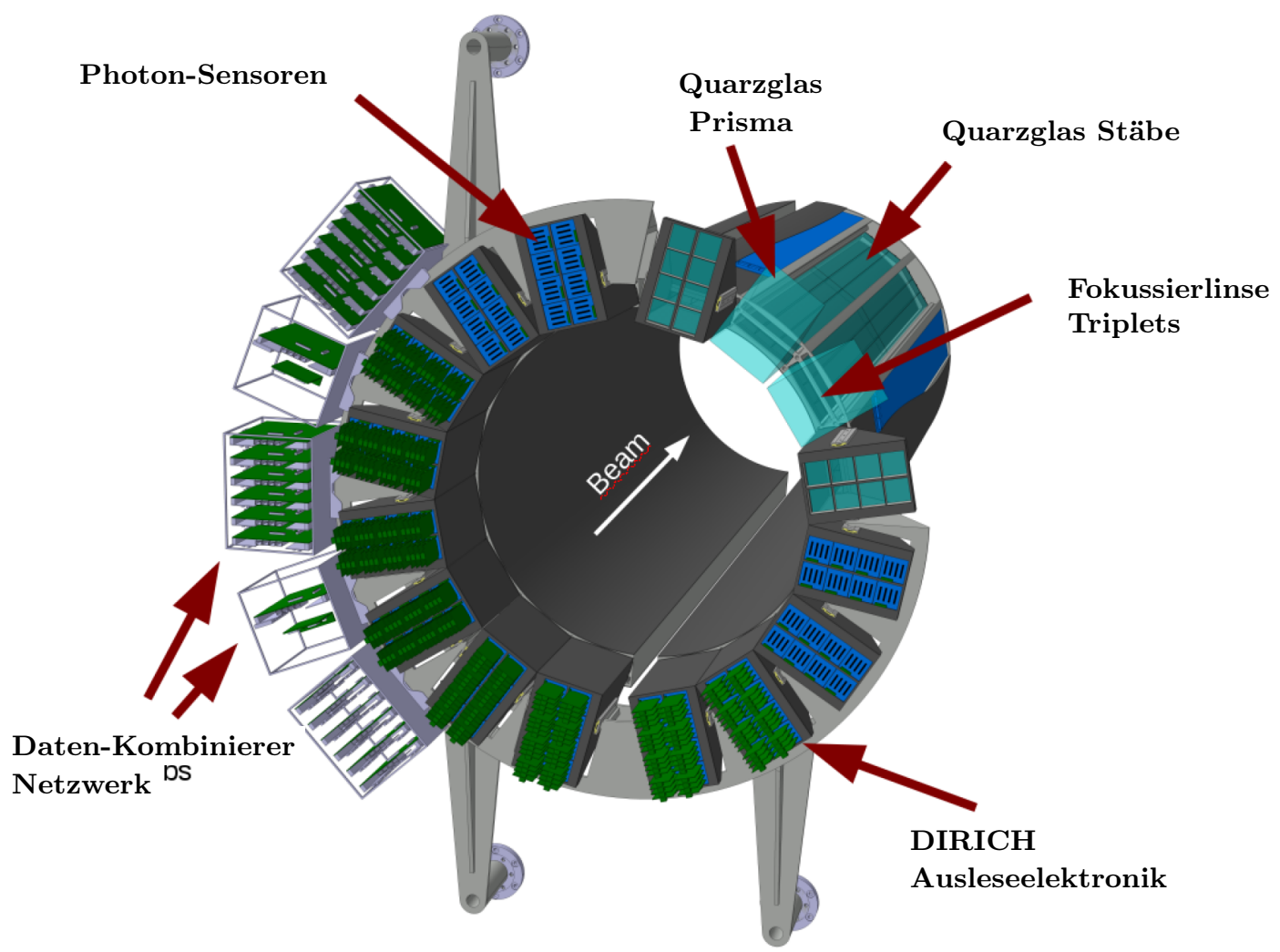

Abbildung 7.1: Schematische Darstellung des PANDA Barrel-DIRC-Designkonzepts [28].

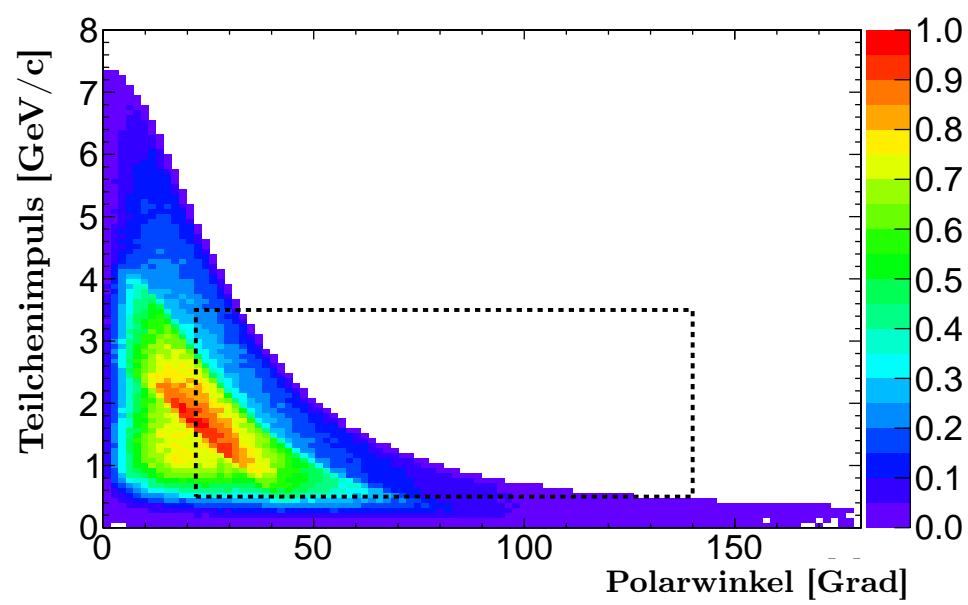

Abbildung 7.2: Phasenraumverteilung von Kaonen für ausgewählte Physikkanäle bei $p_{\bar{p}}=7 \mathrm{GeV} / c$. Der Impuls-Polarwinkelbereich des Barrel DIRC ist mit dem gestrichelten Rechteck markiert [15]. 
Der geometrische Rekonstruktionsansatz bestimmt den Cherenkov-Winkel, indem er sich hauptsächlich auf die Position der detektierten Photonen stützt. Die CherenkovWinkeldaten-Wahrscheinlichkeitsdichtefunktion Methode hängt viel weniger von der richtigen Ausrichtung ab und erzielt vergleichbare Resultate wie die geometrische Standardrekonstruktion jedoch ohne deren Abhängigkeit von optimierten Korrekturprozeduren. Die zeitabbildende Methode optimiert die Verwendung der Positionsund Zeitmessungen der nachgewiesenen Cherenkov-Photonen indem die Zeitsignale direkt mit den Erwartungen für die verschiedenen Teilchensorten verglichen werden um die PID-Information zu bestimmen. Diese Algorithmen erlauben die Bestimmung der wichtigsten Leistungsmerkmale der DIRC-Detektoren: die CherenkovWinkelauflösung pro Photon, die Photonenausbeute und die $\pi / \mathrm{K}$ Trennleistung.

Eine detaillierte Monte-Carlo-Simulation des PANDA Barrel DIRC wurde [31] in Geant4 [32] entwickelt. Diese Simulation beinhaltet die erwarteten technischen Eigenschaften der MCP-PMTs und der Optik (Stäbe, Linsen, Prismen, Spiegel), sowie der Ausleseelektronik, und wurde eingesetzt um den Detektor zu entwerfen, die Leistung zu optimieren und die Systemkosten zu reduzieren. Die simulierte $\pi / K$ Trennleistung des endgültigen Designs mit drei Stäben pro Sektor, dreilagigen sphärischen Linsen und einem Prisma mit 8 Microchannel-Plate Photomultipliern (MCPPMTs) ist als Funktion des Teilchenimpulses und des Polarwinkels in Abb. 7.3 unter Verwendung der zeitabbildenden PID-Methode dargestellt. Mit einer Trennleistung von 4-14 s.d. übertrifft das Design die PANDA PID-Anforderung für den gesamten Phasenraum geladener Kaonen in PANDA.

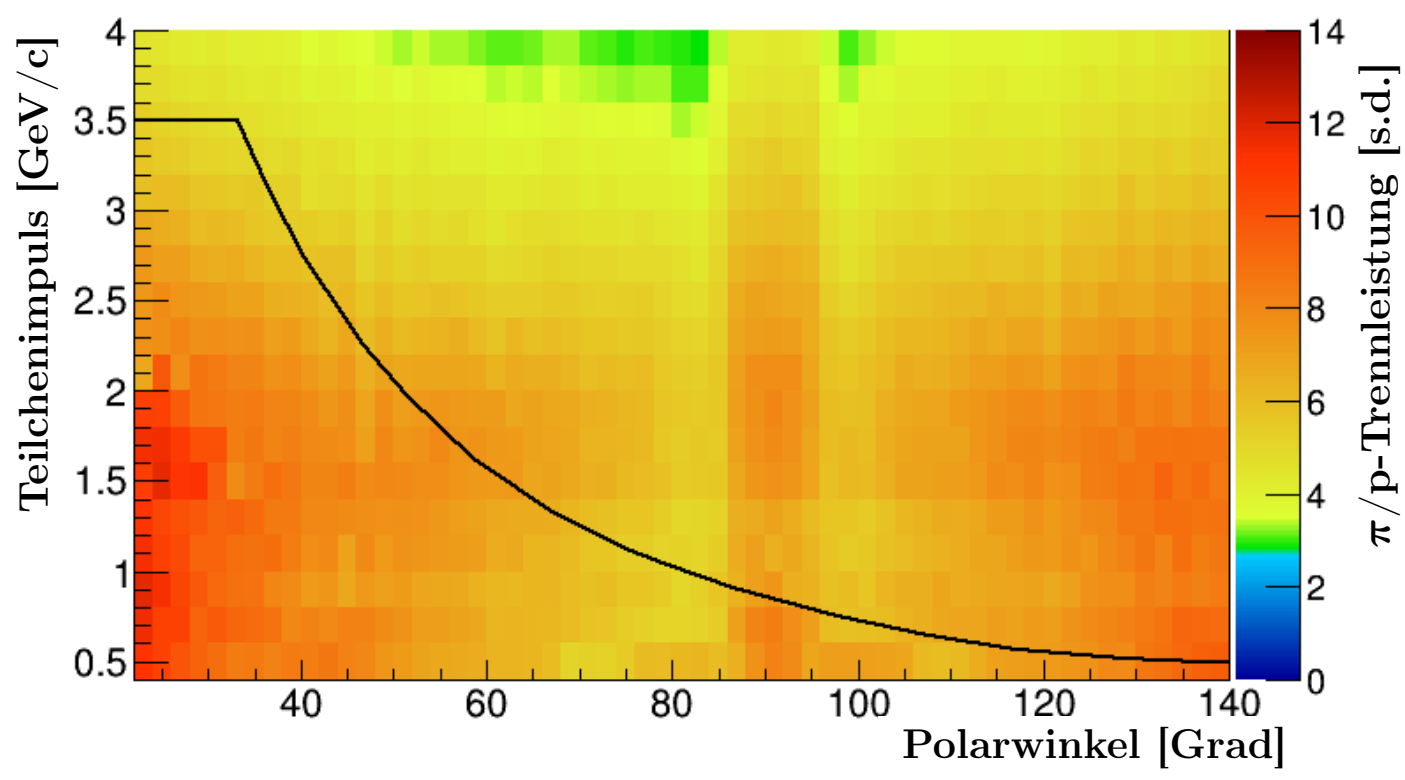

Abbildung 7.3: $\pi / K$-Trennleistung des PANDA Barrel DIRC, bestimmt durch die zeitbasierte bildgebende Methode, als Funktion des Teilchenimpulses und Polarwinkels in der Geant Simulation. Der Bereich unterhalb der schwarzen Linie entspricht dem Endzustandsphasenraum für geladene Kaonen aus verschiedenen Kanälen [15].

Das Ziel des Prototyp-Programms war die experimentelle Bestätigung der von der 
Simulation vorhergesagten Leistung. Das nahezu endgültige Design des PANDA Barrel DIRC wurde im Prototyp implementiert und 2018 mit hadronischen Teilchenstrahlen getestet, um das Design und verschiedene Rekonstruktions- und PIDMethoden zu validieren. Der Prototyp wurde im gemischten Hadronenstrahl in der T9-Zone am CERN PS platziert. Da der Teilchenstrahl hauptsächlich aus Pionen und Protonen bestand war die direkte Bewertung der $\pi$ /K-Trennung nicht möglich. Stattdessen wurde die PID-Leistung mit Pionen und Protonen ausgewertet, die durch ein Flugzeitspektrometer identifiziert wurden. Die Cherenkov-Winkeldifferenz für Pionen und Protonen bei $7 \mathrm{GeV} / \mathrm{c}$ ist ungefähr die gleiche wie für Pionen und Kaonen bei 3,5 GeV/c, der oberen Grenze des PID-Leistungsbereichs des PANDA Barrel DIRC.

Die Performanz des Prototyps wurde in Bezug auf die Photonenausbeute, die EinzelphotonenCherenkov-Winkelauflösung und die $\pi / p$-Trennleistung charakterisiert. Die gemessene Trennleistung ist für drei Rekonstruktionsmethoden in Abb. 7.4-7.6 als Funktion des Polarwinkels für die Simulationen und Experiment Daten mit Hadronenstrahlen dargestellt.

\section{Geometrische Rekonstruktion}

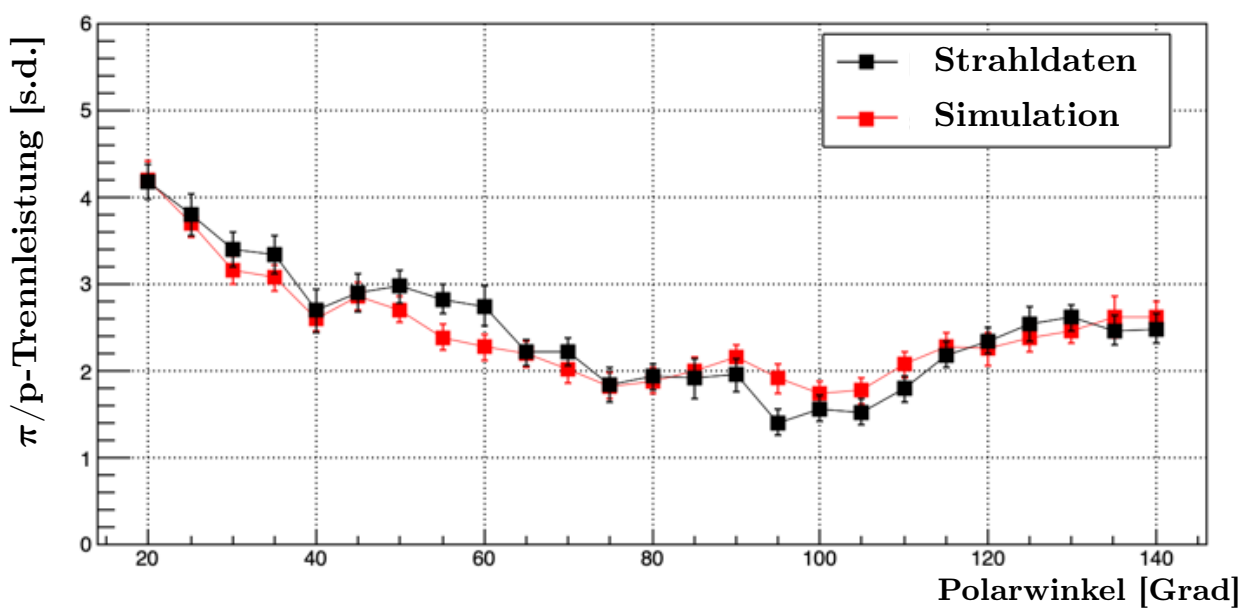

Abbildung 7.4: $\pi / \mathrm{p}$-Trennleistung aus der geometrischen Rekonstruktion als Funktion des Polarwinkels bei einem Impuls von $7 \mathrm{GeV} / \mathrm{c}$ für Strahldaten (schwarz) und Geant4 Simulation (rot).

Die Ergebnisse der Strahlzeit zeigen $\pi$ /p-Separationswerte von bis zu 5,0 $\pm 0,2$ s.d. bei $7 \mathrm{GeV} / \mathrm{c}$, entsprechend $5,2 \pm 0,2$ s.d. für die $\pi / \mathrm{K}$ Trennung bei $3,5 \mathrm{GeV} / \mathrm{c}$. Die besten Resultate wurden durch Verwendung der zeitabbildenden PID-Methode erzielt und übertreffen die PANDA PID-Anforderungen bei diesem Winkel. Die Analyseergebnisse der Daten des Prototyp-Tests am CERN zeigen eine hervorragende Übereinstimmung mit der Simulation, und dadurch auch die PANDA Barrel DIRC Geant4-Simulation validieren.

Das GlueX-Experiment am Jefferson Lab wurde im Jahr 2019 durch den Einbau eines DIRC-Detektors verbessert. Als Teil des FAIR Phase-0-Programms wurde die für den PANDA Barrel DIRC entwickelte Simulation und die Rekonstruktions- und PID-Methoden beim GlueX DIRC-Detektor eingesetzt. Im Rahmen dieser Arbeit wurden die Daten der Inbetriebnahmetests im Dezember 2019 ausgewertet. Dazu wurden kinematisch identifizierte Pionen und Kaonen aus den Zerfällen von $\rho$ - 


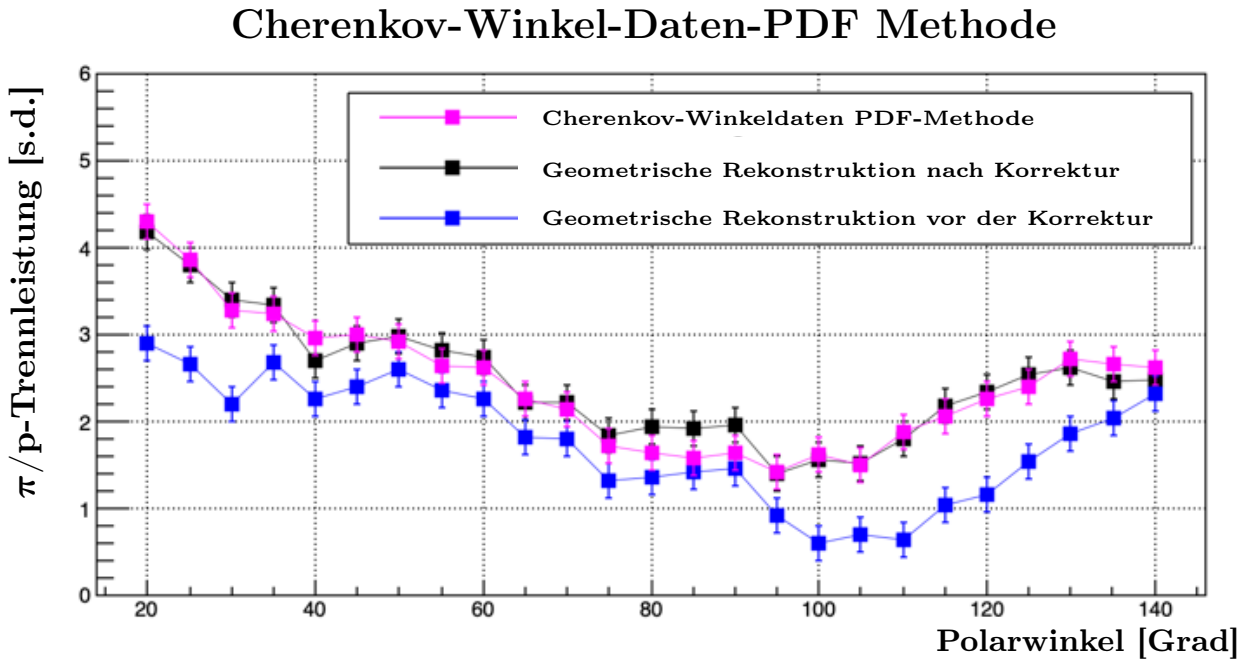

Abbildung 7.5: $\pi /$ p-Trennleistung als Funktion des Polarwinkels bei einem Impuls von $7 \mathrm{GeV} /$ c für Strahldaten unter Benutzung von: der geometrischen Rekonstruktion ohne Korrekturen (blau), der geometrischen Rekonstruktion nach Anwendung aller Korrekturen (schwarz) und der Cherenkov-Winkel-Daten-PDF Methode (magenta).

\section{Zeitabbildenden Methode}

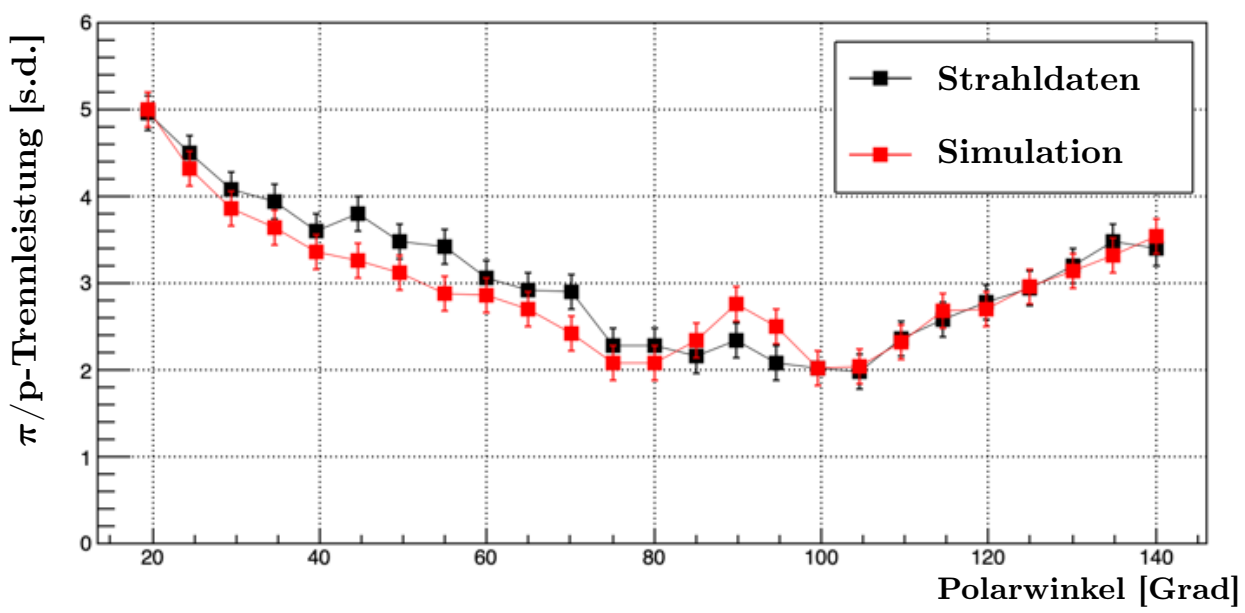

Abbildung 7.6: $\pi$ /p-Trennleistung aus der zeitabbildenden Methode als Funktion des Polarwinkels bei einem Impuls von $7 \mathrm{GeV} / \mathrm{c}$ für Strahldaten (schwarz) und für die Simulation (rot). 
und $\phi$-Mesonen verwendet. Die Ergebnisse zeigen, dass die beobachteten CherenkovMuster und die durch die geometrische Rekonstruktionsmethode bestimmte Auflösung des Cherenkov-Winkels pro Photon mit den Erwartungen durch die MonteCarlo-Simulationen übereinstimmen. Allerdings liegen die Anzahl der CherenkovPhotonen pro Teilchen und die $\pi /$ K-Trennleistung signifikant unter den Geant4Vorhersagen. Für die Anwendung der zeitabbildenden PID-Methode sind die erfassten Daten unzureichend und dadurch ist der Einsatz der Cherenkov-WinkeldatenPDF-Methode auf einen kleinen Phasenraumbereich begrenzt.

Die erzielten Ergebnisse für die geometrische Rekonstruktion sind jedoch sehr vielversprechend, mit bis zu 3 s.d. $\pi$ /K-Separation bei einem Impuls von 3.0-3.5 GeV/c, wie in Abb. $7.7 \mathrm{zu}$ sehen. Es ist zu erwarten, dass sich das Detektionsvermögen des GlueX DIRC-Detektors weiter steigern wird, sobald verbesserte Methoden zur Kalibrierung und Ausrichtung der Detektorkomponenten mit einem grösseren Datensatz durchgeführt werden.

$[3-3,5] \mathrm{GeV} / \mathrm{c}$ Simulation

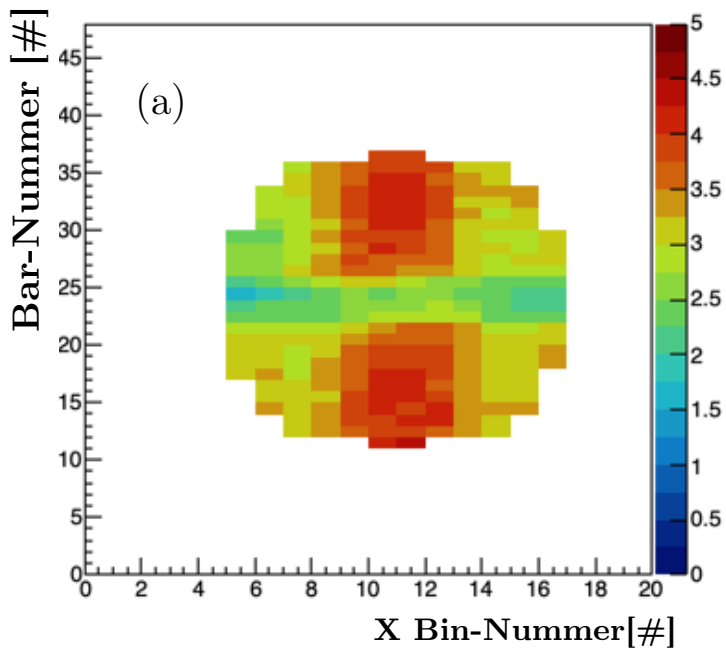

$[3-3,5] \mathrm{GeV} / \mathrm{c}$ Strahldaten

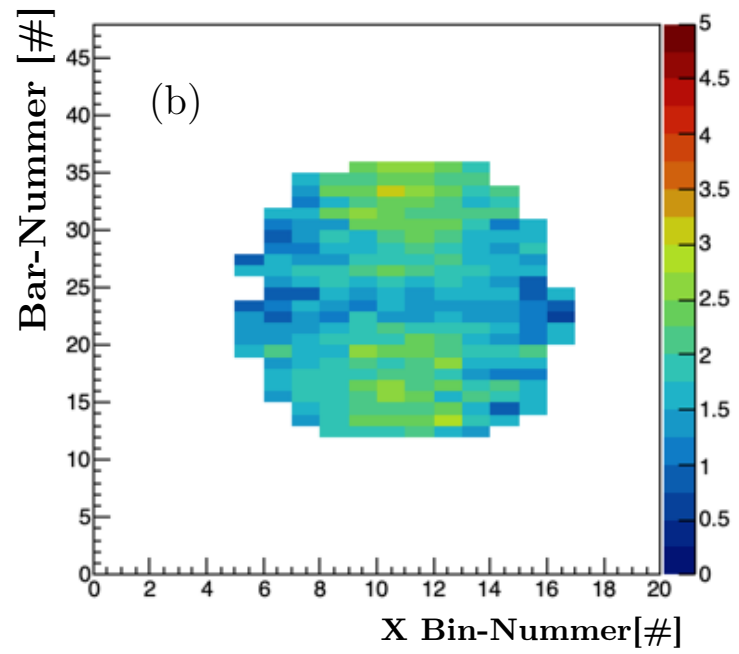

Abbildung 7.7: Trennleistungskarten für Pion/Kaon, identifiziert durch die $\rho$ - und $\phi$-Reaktionen bei Impulsen zwischen 3-3,5 GeV/c, für Simulation (a) und Strahldaten (b).

Der Vergleich der Leistungsfähigkeit der Rekonstruktions- und PID-Methoden mit Simulationen und Strahlzeitdaten war ein wichtiger Teil der Validierung des endgültigen PANDA Barrel DIRC-Designs. Dadurch wurde der Weg zur Serienproduktion der Barrel-DIRC-Komponenten geebnet, die derzeit im Gange ist. Die Wahl der optimalen Algorithmen wird auch in Zukunft ein wichtiger Aspekt für die nächste Generation von DIRC-Zählern sein, wie z. B. den Barrel DIRC für den zukünftigen Elektronen-Ionen-Collider-Detektor, der durch eine noch bessere Zeit- und Positionsauflösung der Cherenkov-Photonen den 3 s.d. $\pi /$ K-Trennbereich auf Impulse von $6 \mathrm{GeV} / \mathrm{c}$ oder höher auszudehnen soll. 


\section{Bibliography}

[1] "FAIR Baseline Technical Report, Volume 2 Accelerator and Scientific Infrastructure". In: (2006). URL: http://repository.gsi.de/record/54068.

[2] D. Ondreka et al. "Recommissioning of SIS18 After FAIR Upgrades". In: 10th International Particle Accelerator Conference. June 2019.

[3] R. Toelle et al. "HESR at FAIR: Status of technical planning". In: Conf. Proc. C 070625 (2007), p. 1442.

[4] A. Belias et al. "FAIR status and the PANDA experiment". In: JINST 15 (2020), p. C10001.

[5] The PANDA experiment at FAIR in Darmstadt home page. Accessed on: 15 Jan 2021. URL: https://panda.gsi.de/.

[6] Miriam Kummel. Physics Prospects of PANDA at FAIR. 9th International Conference on New Frontiers in Physics. 2020. URL: https://indico.cern. ch/event/868045/contributions/3996081/attachments/2097971/3526570/ ICNFP2020_Kuemmel.pdf.

[7] W. Erni et al. "Technical Design Report for the PANDA Internal Targets ". In: (2012). URL: https://panda.gsi.de/system/files/user_uploads/u. kurilla/RE-TDR-2012-002.pdf.

[8] M. F. M. Lutz et al. "Physics Performance Report for PANDA: Strong Interaction Studies with Antiprotons". In: (2009). arXiv: 0903.3905 [hep-ex].

[9] A. Pyszniak et al. "A pellet tracking system for the PANDA experiment". In: Hyperfine Interact. 229 (2014), pp. 159-163.

[10] E. Kohler. "Design and performance of the future cluster-jet target for PANDA at FAIR". In: PoS STORI11 (2011), p. 063.

[11] W. Erni et al. "Technical Design Report for the PANDA Solenoid and Dipole Spectrometer Magnets". In: (2009). arXiv: 0907.0169 [physics.ins-det].

[12] W. Erni et al. "Technical Design Report for the: PANDA Micro Vertex Detector". In: (2012). arXiv: 1207.6581 [physics.ins-det].

[13] W. Erni et al. "Technical design report for the PANDA (AntiProton Annihilations at Darmstadt) Straw Tube Tracker". In: Eur. Phys. J. A 49 (2013).

[14] W. Erni et al. "Technical Design Report for PANDA Electromagnetic Calorimeter (EMC)". In: (2008). arXiv: 0810.1216 [physics.ins-det].

[15] B. Singh et al. "Technical Design Report for the PANDA Barrel DIRC Detector". In: J. Phys. G 46 (2019), p. 045001. 
[16] F. Davi et al. "Technical Design Report for the PANDA Endcap Disc DIRC". In: (2019). arXiv: 1912.12638 [physics.ins-det].

[17] S. Zimmermann et al. "Technical Design Report for the: PANDA Barrel Timeof-Flight". In: (2018). URL: https://panda.gsi.de/system/files/user uploads/ken. suzuki/RE-TDR-2016-003_0.pdf.

[18] W. Erni et al. "Technical Design Report for the PANDA Muon System". In: (2012). URL: https: / / panda.gsi.de/system/files/user_uploads/u . kurilla/RE-TDR-2012-003.pdf.

[19] B. Singh et al. "Technical Design Report for the PANDA Forward Tracker". In: (2018). URL: https: / / panda.gsi.de/system/files/user_uploads / admin/RE-TDR-2017-001.pdf.

[20] B. Singh et al. "Technical Design Report for the PANDA Forward Time-ofFlight Detector". In: (2018). URL: https://panda.gsi.de/system/files/ user_uploads/admin/RE-TDR-2016-004.pdf.

[21] B. Singh et al. "Technical Design Report for the PANDA Forward Spectrometer Calorimeter". In: (2017). arXiv: 1704.02713 [physics.ins-det].

[22] M. Fritsch et al. "Technical Design Report for the PANDA Luminosity Detector". In: (2018). URL: https : / / panda.gsi .de/system/files/user_ uploads/m.fritsch/RE-TDR-2015-001_1.pdf.

[23] P. Coyle et al. "The DIRC counter: A New type of particle identification device for B factories". In: Nucl. Instrum. Meth. A 343 (1994), pp. 292-299.

[24] I. Adamzz et al. "The DIRC particle identification system for the BaBar experiment". In: Nucl. Instrum. Meth. A 538 (2005), p. 281.

[25] B. Dey et al. "Design and performance of the Focusing DIRC detector". In: Nucl. Instrum. Meth. A 775 (2015), pp. 112-131. arXiv: 1410.0075 [physics.ins-det].

[26] K. Inami et al. "TOP counter for particle identification at the Belle II experiment". In: Nucl. Instrum. Meth. A 766 (2014), pp. 5-8.

[27] J. Stevens et al. "The GlueX DIRC Project". In: JINST 11 (2016), p. C07010. arXiv: 1606.05645 [physics.ins-det].

[28] C. Schwarz et al. "Status of the PANDA Barrel DIRC". In: JINST 15 (2020), p. C03055.

[29] Epotek. 14 Fortune Drive, Billerica, MA 01821, USA. URL: http : / / www . epotek.com/site/administrator / components / com_products / assets / files/Style_Uploads/301-2RevXII.pdf.

[30] Momentive Performance Materials Inc. 260 Hudson River Road, Waterford, NY 12188, USA. URL: https://www.momentive.com/en-us.

[31] R. Dzhygadlo et al. "Simulation and reconstruction of the PANDA Barrel DIRC". In: Nucl. Instrum. Meth. A 766 (2014), pp. 263-266.

[32] S. Agostinelli et al. "GEANT4-a simulation toolkit". In: Nucl. Instrum. Meth. A 506 (2003), pp. 250-303.

[33] A. Ali et al. "Particle identification algorithms for the PANDA Barrel DIRC". In: JINST 15 (2020), pp. C09057-C09057. 
[34] M. Staric et al. "Pattern recognition for the time-of-propagation counter". In: Nucl. Instrum. Meth. A 639 (2011), pp. 252-255.

[35] R. Dzhygadlo et al. "Time imaging reconstruction for the PANDA Barrel DIRC". In: JINST 15 (2020), p. C09050.

[36] L. Durieu et al. "Optics Studies for the T9 Beam Line in the CERN PS East Area Secondary Beam Facility". In: Conf. Proc. C 0106181 (2001), pp. 15471549.

[37] Photonis USA Pennsylvania Inc. 1000 New Holland Avenue Lancaster PA 17601-5688 USA. URL: https://www . photonis . com/our-company-companies/ photonis-defense-inc.

[38] Eljen Technology. 1300 W. Broadway, Sweetwater Texas 79556, USA. URL: http://www . eljentechnology . com/products/accessories/ej-550-ej552.

[39] M. Traxler et al. "A compact system for high precision time measurements ( 14 ps RMS) and integrated data acquisition for a large number of channels". In: JINST 6 (2011), p. C12004.

[40] H. Alvarez-Pol et al. "A large area timing RPC prototype for ion collisions in the HADES spectrometer". In: Nucl. Instrum. Meth. A 535 (2004), pp. 277282.

[41] C. Ugur et al. "Field programmable gate array based data digitisation with commercial elements". In: JINST 8 (2013), pp. C01035-C01035.

[42] Advanced Laser Diode Systems A.L.S GmbH. Schwarzschildstr. 6, D-12489 Berlin, Germany. URL: http://www.alsgmbh.com/.

[43] R. Brun et al. "ROOT: An object oriented data analysis framework". In: Nucl. Instrum. Meth. A 389 (1997), pp. 81-86.

[44] E. Pooser. "The GlueX Start Counter Beam Asymmetry $\Sigma$ in Single $\pi^{0}$ Photoproduction". PhD thesis. Florida International University, 2016.

[45] Private communication - C. Schwarz.

[46] M. Patsyuk. "Simulation, Reconstruction, and Design Optimization for the PANDA Barrel DIRC". PhD thesis. Goethe-Universität Frankfurt am Main, 2014.

[47] S. Adhikari et al. "The GlueX beamline and detector". In: Nucl. Instrum. Meth. A 987 (2021), p. 164807.

[48] J. Dudek et al. "Toward the excited isoscalar meson spectrum from lattice QCD". In: Phys. Rev. D 88.9 (2013), p. 094505. arXiv: 1309.2608 [hep-lat].

[49] Thomas Jefferson National Accelerator Facility (JLab) 12 GeV upgrade website. Accessed on: 10 Sep 2020. URL: https://www.jlab.org/physics/GeV.

[50] Thomas Jefferson National Accelerator Facility (JLab) Hall D website. Accessed on: 10 Sep 2020. URL: https://www. jlab.org/Hall-D/.

[51] H. Al Ghoul et al. "First Results from The GlueX Experiment". In: AIP Conf. Proc. 1735.1 (2016), p. 020001. arXiv: 1512.03699 [nucl-ex].

[52] A. Ali et al. "Installation and Commissioning of the GlueX DIRC". In: JINST 15.09 (2020), p. C09010. arXiv: 2005.07195 [physics.ins-det]. 
[53] J. Dudek et al. "Mapping the Spectrum of Light Quark Mesons and Gluonic Excitations with Linearly Polarized Photons Presentation to PAC 30". In: 2006. URL: http://www.gluex.org/docs/pac30proposal.pdf.

[54] A. Ali et al. "The GlueX DIRC Program". In: JINST 15.04 (2020), p. C04054. arXiv: 2002.07990 [physics.ins-det].

[55] J. Hardin. "Upgrading particle identification and searching for leptophobic bosons at GlueX." PhD thesis. Massachusetts Institute of Technology, 2018.

[56] M. Contalbrigo et al. "Single photon detection with the multi-anode CLAS12 RICH detector". In: Nucl. Instrum. Meth. A 952.09 (2020), p. 162123.

[57] I. Adam et al. "The DIRC particle identification system for the BaBar experiment". In: Nucl. Instrum. Meth. A 538 (2005), pp. 281-357.

[58] F. Barbosa et al. "The GlueX DIRC detector". In: Nucl. Instrum. Meth. A 876 (2017), pp. 69-71.

[59] M. Patsyuk et al. GlueX internal notes (DocDB 3974, 2019.)

[60] Hamamatsu. 360 Foothill Road, Bridgewater, NJ 08807, USA. URL: https: //www.hamamatsu.com/jp/en/product/type/H12700B/index.html.

[61] M. Contalbrigo et al. "The CLAS12 large area RICH detector". In: Nucl. Instrum. Meth. A 639 (2011), pp. 302-306.

[62] S. Blin et al. "MAROC, a generic photomultiplier readout chip". In: IEEE Nucl. Sci. Symp. Conf. Rec. 2010 (2010), pp. 1690-1693.

[63] M. Contalbrigo et al. "Single photon detection with the multi-anode CLAS12 RICH detector". In: Nucl. Instrum. Meth. A 952 (2020), p. 162123.

[64] J. Hardin and Mike Williams. "FastDIRC: a fast Monte Carlo and reconstruction algorithm for DIRC detectors". In: JINST 11.10 (2016), P10007. arXiv: 1608.01180 [physics.data-an].

[65] Y. Yang. "Commissioning the DIRC Detector and Searching for Axion-like Particles at GlueX." PhD thesis. Massachusetts Institute of Technology, 2021.

[66] Private communication - J. Schwiening. 